Supporting Information

\title{
Chlorinative Cyclization of Aryl Alkynoates using NCS and 9-Mesityl-10- Methylacridinium Perchlorate Photocatalyst
}

Milan Pramanik, ${ }^{a}$ Ashis Mathuri, ${ }^{a}$ Sudip Sau, ${ }^{a}$ Monojit Das ${ }^{a}$ and Prasenjit Mal ${ }^{* a}$

${ }^{a}$ School of Chemical Sciences, National Institute of Science Education and Research (NISER)

Bhubaneswar, HBNI, PO Bhimpur-Padanpur, Via Jatni, District Khurda, Odisha 752050, India.

*Corresponding author: Prasenjit Mal, Tel.: +91 9439613856, E-mail: pmal@niser.ac.in

\section{Content}

General Information

Synthesis and control experiments

S2-S11

The reaction condition optimization (Table $\mathrm{S} 1$ )

$\mathrm{S} 12$

Compound characterization data

S13-S34

References

S34-S35

NMR spectra

S36-S91

Unsuccessful substrates (Chart 1)

S91

ESI-MS data of the experiment using DMPO

S92

Photo reactor details

S93-S94 


\section{EXPERIMENTAL SECTION}

General Aspects. All the chemicals were purchased from commercial sources and used as received. Generally, all the reactions were carried out under an open atmosphere unless otherwise noted. The reactions were monitored by TLC on aluminum sheets pre-coated with silica gel. Chromatographic purifications of the compounds were performed using silica gel (Mess 230-400) and ethyl acetate/hexane as eluent. ${ }^{1} \mathrm{H}$ and ${ }^{13} \mathrm{C}$ spectra were recorded on Bruker 400 and $700 \mathrm{MHz}$ instruments at $25^{\circ} \mathrm{C}$. The chemical shift value $(\delta, \mathrm{ppm})$ was reported to the residual chloroform (7.26 for ${ }^{1} \mathrm{H}$ and $77.16 \mathrm{ppm}$ for $\left.{ }^{13} \mathrm{C}\right)$ and DMSO-d $\mathrm{d}_{6}\left(2.50\right.$ for ${ }^{1} \mathrm{H}$ and 39.52 ppm for ${ }^{13} \mathrm{C}$ ). Mass spectra were recorded as ESI-TOF (HRMS). Infrared spectra were recorded on neat solids using $\mathrm{KBr}$ pellets and described in wavenumber $\left(\mathrm{cm}^{-1}\right)$. Digital melting point apparatus was used to record the Melting Point of the compound in degree centigrade $\left({ }^{\circ} \mathrm{C}\right)$ and are uncorrected.

\section{SYNTHESIS}

Representative procedure for the synthesis of aryl 3-phenylpropiolate derivatives. ${ }^{1}$ In a round-bottomed flask, a solution of phenyl propiolic acid $(5.81 \mathrm{mmol}, 1.1$ equiv) was made by the addition of $10 \mathrm{~mL} \mathrm{CH} \mathrm{Cl}_{2}(\mathrm{DCM})$; followed by the solution was allowed to stir at $-20{ }^{\circ} \mathrm{C}$. After that, a mixture of 4-dimethylaminopyridine $(0.53 \mathrm{mmol}, 0.1$ equiv $)$ and dicyclohexylcarbodiimide (5.81 mmol, 1.1 equiv) in $5 \mathrm{~mL} \mathrm{CH}_{2} \mathrm{Cl}_{2}$ was slowly added to the propiolic acid solution. Again, a solution of aniline (5.31 mmol, 1.0 equiv) in $5 \mathrm{~mL} \mathrm{CH}_{2} \mathrm{Cl}_{2}$ was then added dropwise. Afterward, the reaction mixture was stirred at room temperature for another 6-12 h. After completion of the reaction, the crude reaction mixture was washed by 0.5 
$\mathrm{M}$ aq. $\mathrm{HCl}$, dried over $\mathrm{Na}_{2} \mathrm{SO}_{4}$, and concentrated under rotary-evaporator. Finally, the crude residue was purified by column chromatography to afford the desired phenylpropiolate derivatives.

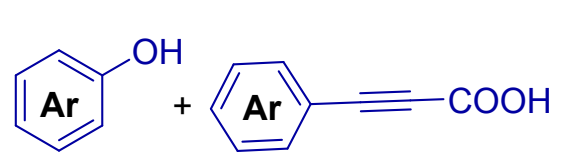

(1.1 equiv)

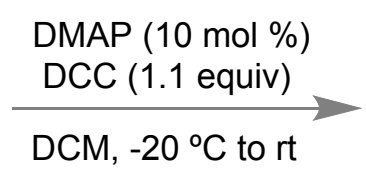

$12 \mathrm{~h}$

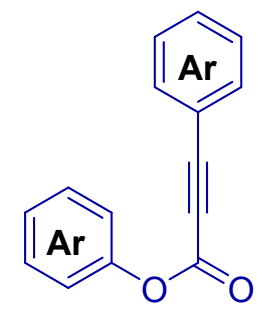

Scheme S1. Synthesis of 1.

Representative procedure for the preparation of 3-chlorocoumarins. In an oven dried quartz tube phenyl 3-phenylpropiolate 1a $(0.270 \mathrm{mmol}, 60 \mathrm{mg}), \mathrm{N}$-chlorosuccinimide (NCS) $(0.810$ mmol, $108 \mathrm{mg}$ ), and Mes-Acr-MeClO $4(5 \mathrm{~mol} \%, 0.0135 \mathrm{mmol}, 6 \mathrm{mg})$ were dissolved in $1.0 \mathrm{~mL}$ dry dichloroethane (DCE) solvent under argon atmosphere. Following, the reaction mixture was degassed for 15 min under argon atmosphere. After that, the reaction mixture was irradiated by $5 \mathrm{~W}$ Blue LEDs $(5 \mathrm{~W} \times 4)$ light (wavelength $450-455 \mathrm{~nm}$ ) for $24 \mathrm{~h}$ in the presence of an argon balloon. After completion of the reaction, DCE was removed under reduced pressure. Then, the crude mixture was diluted in chloroform $\left(\mathrm{CHCl}_{3}\right)$ and extracted with saturated sodium thiosulphate solution to remove excess NCS from the reaction mixture. The resulting organic solution was dried over anhydrous sodium sulfate and concentrated to obtain a crude mixture which was further purified by silica-gel column chromatography using distilled ethyl acetate and hexane as the eluent to afford the pure product. 
Synthetic procedure for compound 5. ${ }^{2}$ To a $2 \mathrm{~mL}$ DMF solution of $2 \mathrm{a}(60 \mathrm{mg}, 0.234 \mathrm{mmol})$, $\mathrm{Et}_{3} \mathrm{SiH}(186 \mu \mathrm{L}, 1.17 \mathrm{mmol})$ and $\mathrm{Et}_{3} \mathrm{~N}(65 \mu \mathrm{L}, 0.468 \mathrm{mmol}), \mathrm{Pd}\left(\mathrm{PPh}_{3}\right)_{4}(54 \mathrm{mg}, 0.0468 \mathrm{mmol})$ was added under argon atmosphere and stirred at $110{ }^{\circ} \mathrm{C}$ in an oil bath for $24 \mathrm{~h}$. After the completion of the reaction, solution was quenched with aq. $\mathrm{NH}_{4} \mathrm{Cl}(5 \mathrm{~mL})$ and then extracted with EtOAc, dried over $\mathrm{Na}_{2} \mathrm{SO}_{4}$ and concentrated in rotary evaporator. The crude mixture was further purified by column chromatography to afford 5 (35 mg, 67\%) as a white solid.

Synthetic procedure for compound $6 .{ }^{3}$ To a $2 \mathrm{~mL} \mathrm{Et}_{3} \mathrm{~N}$ solution of $2 \mathrm{a}(60 \mathrm{mg}, 0.234 \mathrm{mmol})$ and phenyl acetylene (39 $\mu \mathrm{L}, 0.351 \mathrm{mmol}), \mathrm{CuI}(8 \mathrm{mg}, 0.0468 \mathrm{mmol})$ and $\mathrm{Pd}\left(\mathrm{PPh}_{3}\right)_{2} \mathrm{Cl}_{2}(8 \mathrm{mg}$, $0.0117 \mathrm{mmol}$ ) were added under argon atmosphere and stirred at $60^{\circ} \mathrm{C}$ in an oil bath for $24 \mathrm{~h}$. After the completion of the reaction, solution was quenched with aq. $\mathrm{NH}_{4} \mathrm{Cl}(5 \mathrm{~mL})$ and then extracted with EtOAc, dried over $\mathrm{Na}_{2} \mathrm{SO}_{4}$ and concentrated in rotary evaporator. The crude mixture was further purified by column chromatography to afford 6 (29 $\mathrm{mg}, 52 \%)$ as a white solid.

Gram-scale synthesis of 3-chlorocoumarins (2a). In an oven dried quartz tube phenyl 3phenylpropiolate 1a (1.036 mmol, $230 \mathrm{mg}), N$-chlorosuccinimide (NCS) $(3.108 \mathrm{mmol}, 413 \mathrm{mg})$, and Mes-Acr- $\mathrm{MeClO}_{4}(5 \mathrm{~mol} \%, 0.0518 \mathrm{mmol}, 22 \mathrm{mg})$ were dissolved in $4.0 \mathrm{~mL}$ dry dichloroethane (DCE) solvent under argon atmosphere. Following, the reaction mixture was degassed for 15 min under argon atmosphere. After that, the reaction mixture was irradiated by $5 \mathrm{~W}$ Blue LEDs $(5 \mathrm{~W} \times 4)$ light for $24 \mathrm{~h}$ in the presence of an argon balloon. After completion of the reaction, DCE was removed under reduced pressure. Then, the crude mixture was diluted in chloroform $\left(\mathrm{CHCl}_{3}\right)$ and extracted with saturated sodium thiosulphate solution to remove excess 
NCS from the reaction mixture. The resulting organic solution was dried over anhydrous sodium sulfate and concentrated to obtain a crude mixture which was further purified by silica-gel column chromatography using distilled ethyl acetate and hexane as the eluent to afford the pure product 2a with 54\% yield (145 $\mathrm{mg})$.

Radical trapping experiment with TEMPO/BHT/Diphenylethelene. In an oven-dried quartz tube phenyl 3-phenylpropiolate 1a $(0.270 \mathrm{mmol}, 60 \mathrm{mg})$, N-chlorosuccinimide (NCS) $(0.810$ mmol, $108 \mathrm{mg})$, and Mes-Acr-MeClO $4(5 \mathrm{~mol} \%, 0.0135 \mathrm{mmol}, 6 \mathrm{mg})$ and TEMPO (0.54 mmol, $84 \mathrm{mg}$ ) were dissolved in $1.0 \mathrm{~mL}$ dry dichloroethane (DCE) solvent. Following, the reaction tube was degassed for $15 \mathrm{~min}$ by argon gas. After that, the reaction mixture was irradiated by $5 \mathrm{~W}$ Blue LEDs $(5 \mathrm{~W} \times 4)$ light for $24 \mathrm{~h}$ in the presence of an argon balloon. The reaction was monitored by TLC. After the reaction time, no desired product was found. The same experiment was carried out using BHT (118 mg, $0.54 \mathrm{mmol})$ and 1,1-diphenylethylene (98 mg, $0.54 \mathrm{mmol})$. However, the addition of BHT led to no product formation whereas diphenylethylene reduced the yield of the product $\mathbf{2 a}$ giving only $11 \%(4 \mathrm{mg})$.

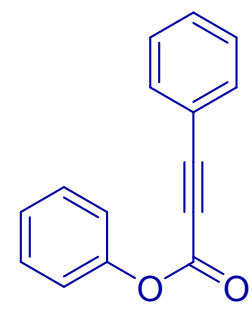

Radical Scavenger (2 equiv) NCS (3.0 equiv) Mes-Acr-MeClO

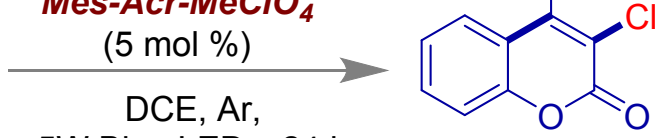
$5 \mathrm{~W}$ Blue LEDs, $24 \mathrm{~h}$

1a, $0.135 \mathrm{mmol}$ Radical Scavenger<smiles>C=C(c1ccccc1)c1ccccc1</smiles>

Diphenylethylene, $11 \%$

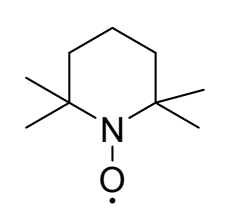

TEMPO, $0 \%$
$2 a$

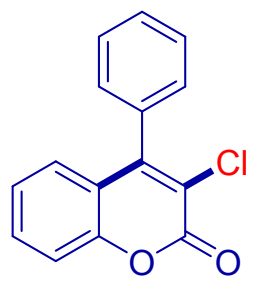<smiles>Cc1cc(C(C)(C)C)c(O)c(C(C)(C)C)c1</smiles>

BHT, 0\% 
Scheme S2. Various radical scavengers under standard condition.

Light ON-OFF-ON Experiment. $P$-Tolyl 3-phenylpropiolate $10(0.270 \mathrm{mmol}, 60 \mathrm{mg})$, NCS $(0.810 \mathrm{mmol}, 108 \mathrm{mg})$ and Mes-Acr- $\mathrm{MeClO}_{4}(5 \mathrm{~mol} \%, 0.0135 \mathrm{mmol}, 6 \mathrm{mg})$ were dissolved in $1.0 \mathrm{~mL}$ dry DCE. Following, the reaction tube was degassed for $15 \mathrm{~min}$ by argon gas. After that, the reaction mixture was irradiated by $5 \mathrm{~W}$ Blue LEDs $(5 \mathrm{~W} \times 4)$ light for $24 \mathrm{~h}$ in the presence of an argon balloon. Successive progress of the reaction was monitored every $6 \mathrm{~h}$ and $4 \mathrm{~h}$ in the presence of light and absence of light by ${ }^{1} \mathrm{H}$ NMR experiment using dibromomethane as an internal standard.

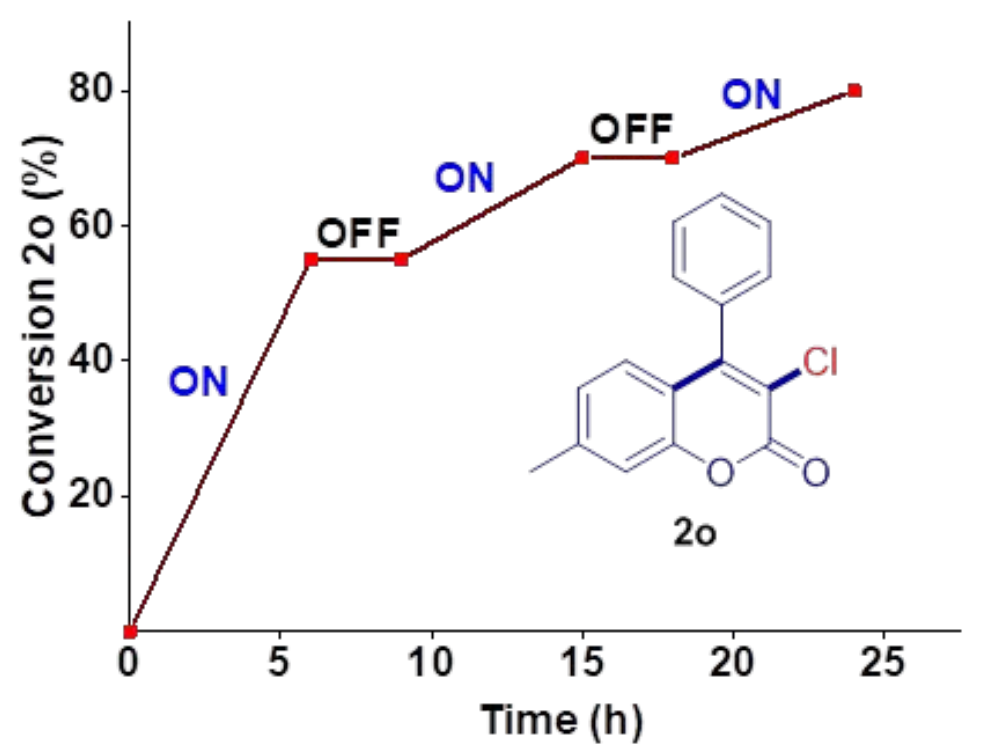

Fig. S1. Conversion of $20 v s$. time in the presence and absence of light.

\section{EPR Experiments.}

EPR spectra was recorded at $298 \mathrm{~K}$ using EPR spectrometer derived at $9.4335 \mathrm{GHz}$. Typical spectrometer parameters are shown as follows, scan range: $100 \mathrm{G}$; center field set: $3480.00 \mathrm{G}$; 
time constant: $0.16 \mathrm{~ms}$; scan time: $122.88 \mathrm{~s}$; modulation amplitude: $20.0 \mathrm{G}$; modulation frequency: $100 \mathrm{kHz}$; receiver gain: $2.00 \times 10^{2}$; microwave power: $7.14 \mathrm{e}^{-001} \mathrm{~mW} ; \mathrm{g}=2.007092$.

Spin-trapping experiment in the presence DMPO. ${ }^{4}$ A mixture of phenyl 3-phenylpropiolate $1 \mathrm{a}(0.270 \mathrm{mmol}, 60 \mathrm{mg})$, NCS $(0.810 \mathrm{mmol}, 108 \mathrm{mg}), \mathrm{Mes}-\mathrm{Acr}-\mathrm{MeClO}_{4}(5 \mathrm{~mol} \%, 0.0135$ mmol, $6 \mathrm{mg}$ ), and 5,5-dimethyl-1- pyrroline-N-oxide (DMPO) $(20 \mu \mathrm{L})$ were stirred in $1.0 \mathrm{~mL}$ dry DCE and after degassing the reaction mixture for $15 \mathrm{~min}$, it was allowed to irradiate in $5 \mathrm{~W}$ Blue LEDs for $6 \mathrm{~h}$. Afterward, $20 \mu \mathrm{L}$ solution was quickly poured into EPR tube and $200 \mu \mathrm{L}$ toluene was appended to analyze EPR. A sharp signal appeared, indicating the presence of an unpaired electron in the reaction pathway. A similar experiment was performed without MesAcr- $\mathrm{MeClO}_{4}$; which shows no signal.

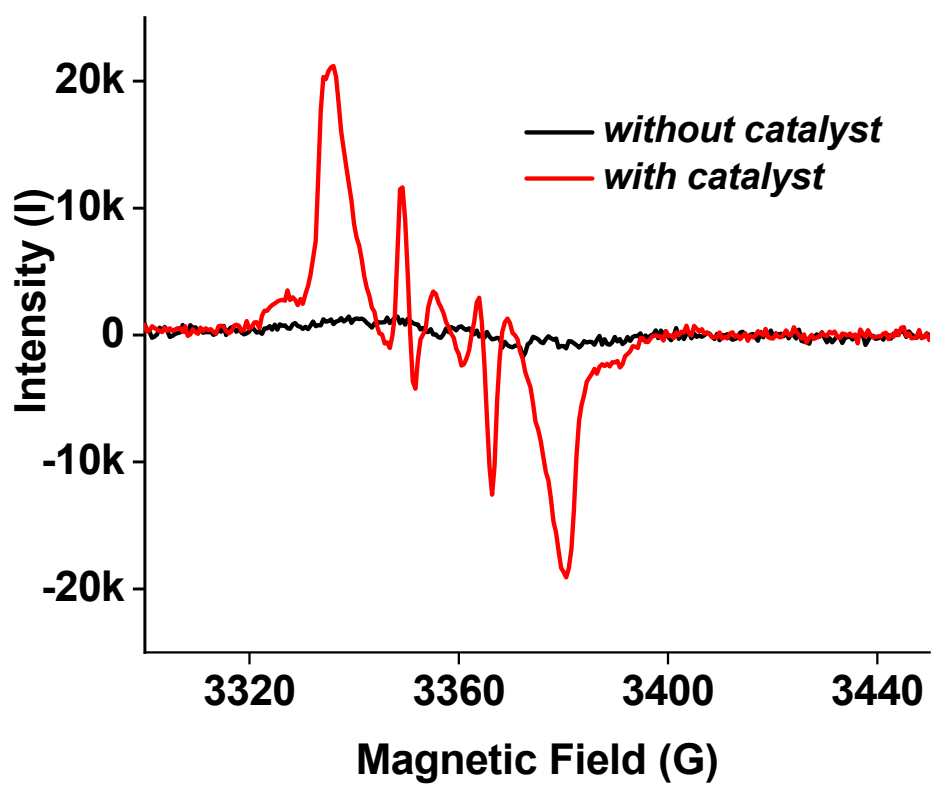

Fig. S2. a) EPR experiment under the standard condition with catalyst (red signal) and without catalyst (black line). 
Fluorescence quenching study. ${ }^{5}$ The maximum emission of the photocatalyst Mes- $\mathrm{Acr}-\mathrm{MeClO}_{4}$ ( $4 \times 10^{-5} \mathrm{M}$ in DCE) was observed at $512 \mathrm{~nm}$ upon excitation wavelength at $350 \mathrm{~nm}$. Following, the addition of $1 \mathrm{a}\left(4 \times 10^{-5} \mathrm{M}\right.$ in DCE) led to the gradual decrease of fluorescence intensity, as shown below.

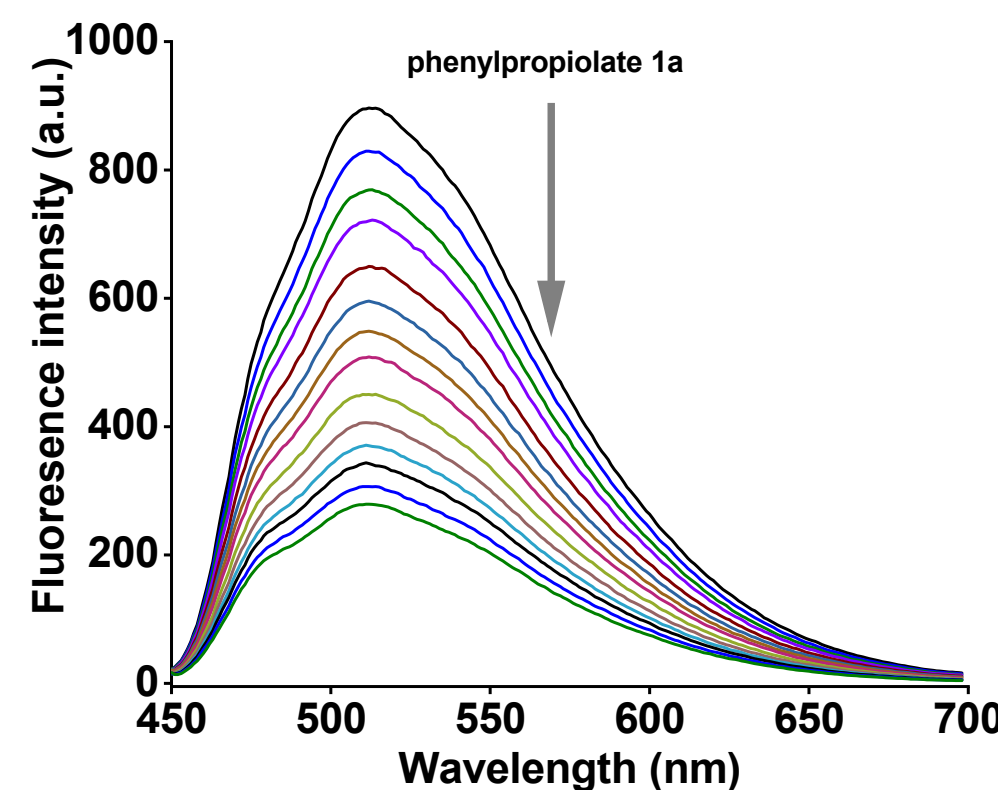

Fig. S3. Fluorescence spectra of Mes-Acr- $\mathrm{MeClO}_{4}$ upon gradual addition of phenylpropiolate 1a. 


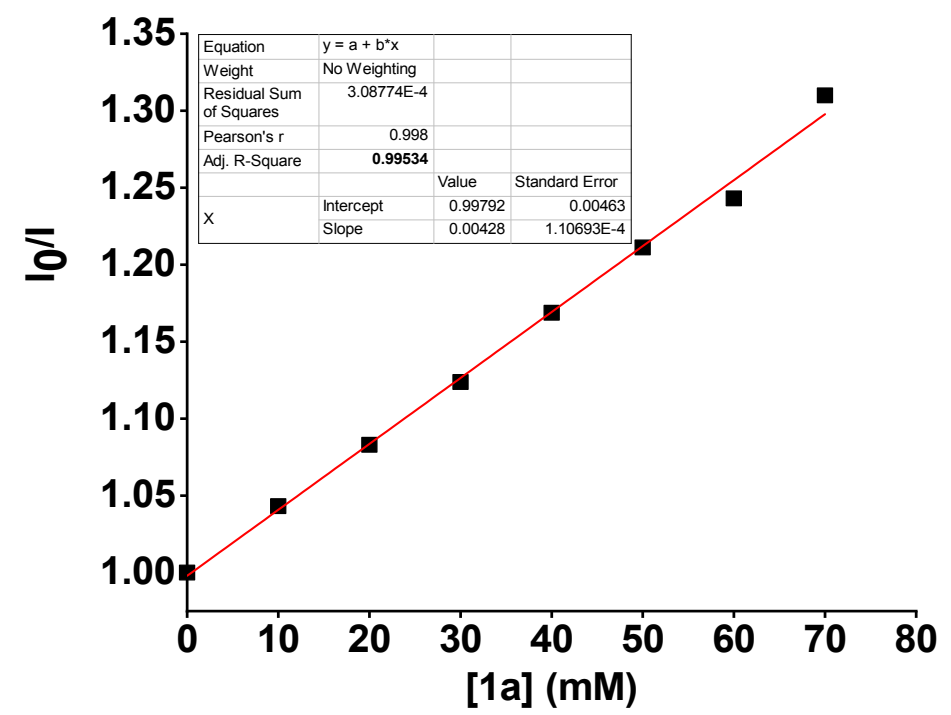

Fig. S4. Stern-Volmer plot for phenylpropiolate $1 \mathbf{a}$.

Cyclic voltammetry experiment. Cyclic voltammetry was carried out using an electrochemical workstation instrument (Corrtest Instrument). Tetrabutylammonium hexafluorophosphate (TBAH) $(0.1 \mathrm{M})$ and phenyl propiolate 1a in DCE solvent using Pt working electrode, Pt wire as a counter electrode and saturated calomel electrode (SCE) as a reference electrode at $100 \mathrm{mV} / \mathrm{s}$ scan rate.

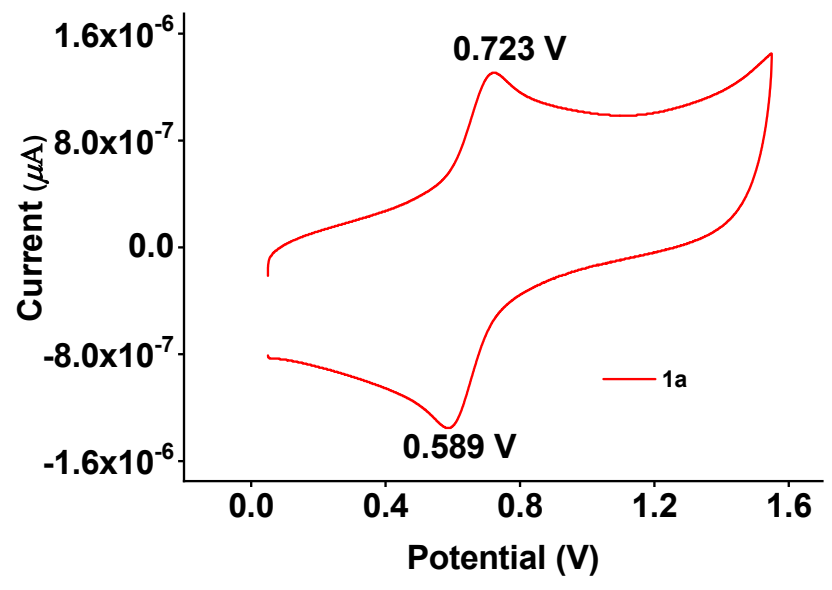

Fig. S5. Cyclic voltammetry of 1a 


\section{Crystal measurement}

Crystals of compound $\mathbf{2 b}$ was achieved after slow evaporation of ethanol and water mixture (1:1). The crystals data were collected with Bruker SMART D8 goniometer equipped with an APEX CCD detector and with an INCOATEC micro source (Mo-K $\alpha$ radiation, $\lambda=0.71073 \AA$ ). SAINT $+^{6}$ and SADABS $^{7}$ were used to integrate the intensities and to correct the absorption respectively The structure was resolved by direct methods and refined on $\mathrm{F}^{2}$ with SHELXL-97. ${ }^{8}$ ORTEP drawing of the compound $\mathbf{2 b}$ show ellipsoid contour at the $50 \%$ probability level.

\section{Compound 2b (CCDC 2097536)}

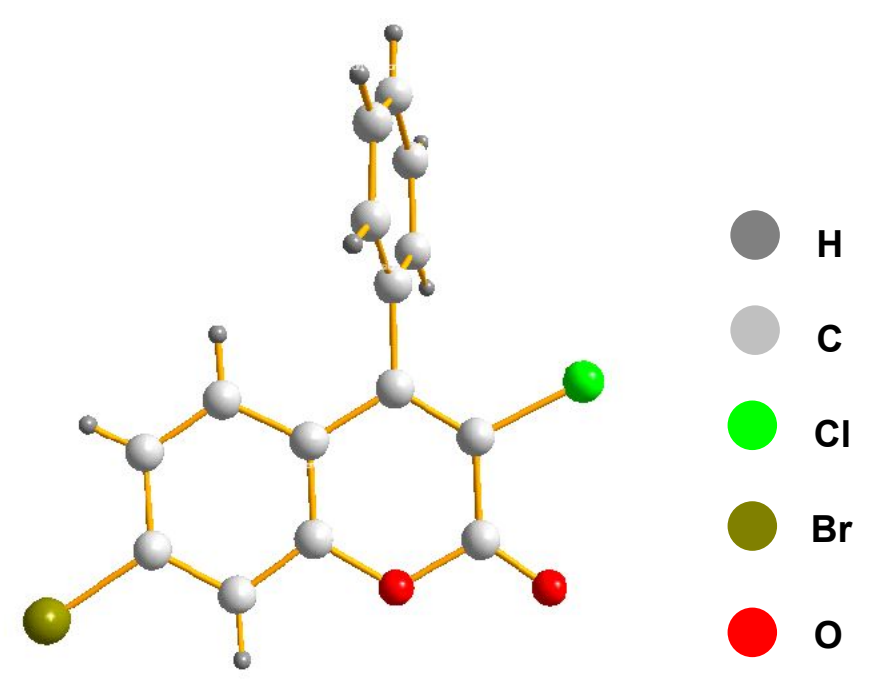

Fig. S6. Crystal structure of $\mathbf{2 b}$ (CCDC 2097536).

\section{Crystallographic Data for (2b)}

Empirical formula

Formula weight

Temperature/K
$\mathrm{C}_{15} \mathrm{H}_{8} \mathrm{BrClO}_{2}$

335.58

293(2) 
Crystal system

Space group

$\mathrm{a} / \AA$

$\mathrm{b} / \AA$

$\mathrm{c} / \AA$

$\alpha /^{\circ}$

$\beta /^{\circ}$

$\gamma /{ }^{\circ}$

Volume/ ̊3

Z

pcalcg $/ \mathrm{cm}^{3}$

$\mu / \mathrm{mm}^{-1}$

$\mathrm{F}(000)$

Crystal size/ $/ \mathrm{mm}^{3}$

Radiation

Reflections collected

Independent reflections

Goodness-of-fit on F2

Final $R$ indexes $[\mathrm{I}>=2 \sigma(\mathrm{I})]$

Final $\mathrm{R}$ indexes [all data]

Largest diff. peak/hole / e $\AA^{-3}$
Monoclinic

$\mathrm{P} 2{ }_{1} / \mathrm{c}$

$10.32340(10)$

$15.4083(2)$

$8.31420(10)$

90

98.8950(10)

90

1306.60(3)

1

1.706

6.123

664.0

$0.2 \times 0.2 \times 0.1$

$\mathrm{CuK} \alpha(\lambda=1.54184)$

47713

$2387[$ Rint $=0.0446$, Rsigma $=0.0162]$

1.115

$\mathrm{R}_{1}=0.0722, \mathrm{wR}_{2}=0.2142$

$\mathrm{R}_{1}=0.0739, \mathrm{wR}_{2}=0.2169$

$0.63 /-1.17$ 
Table S1. The reaction condition optimization. ${ }^{a}$

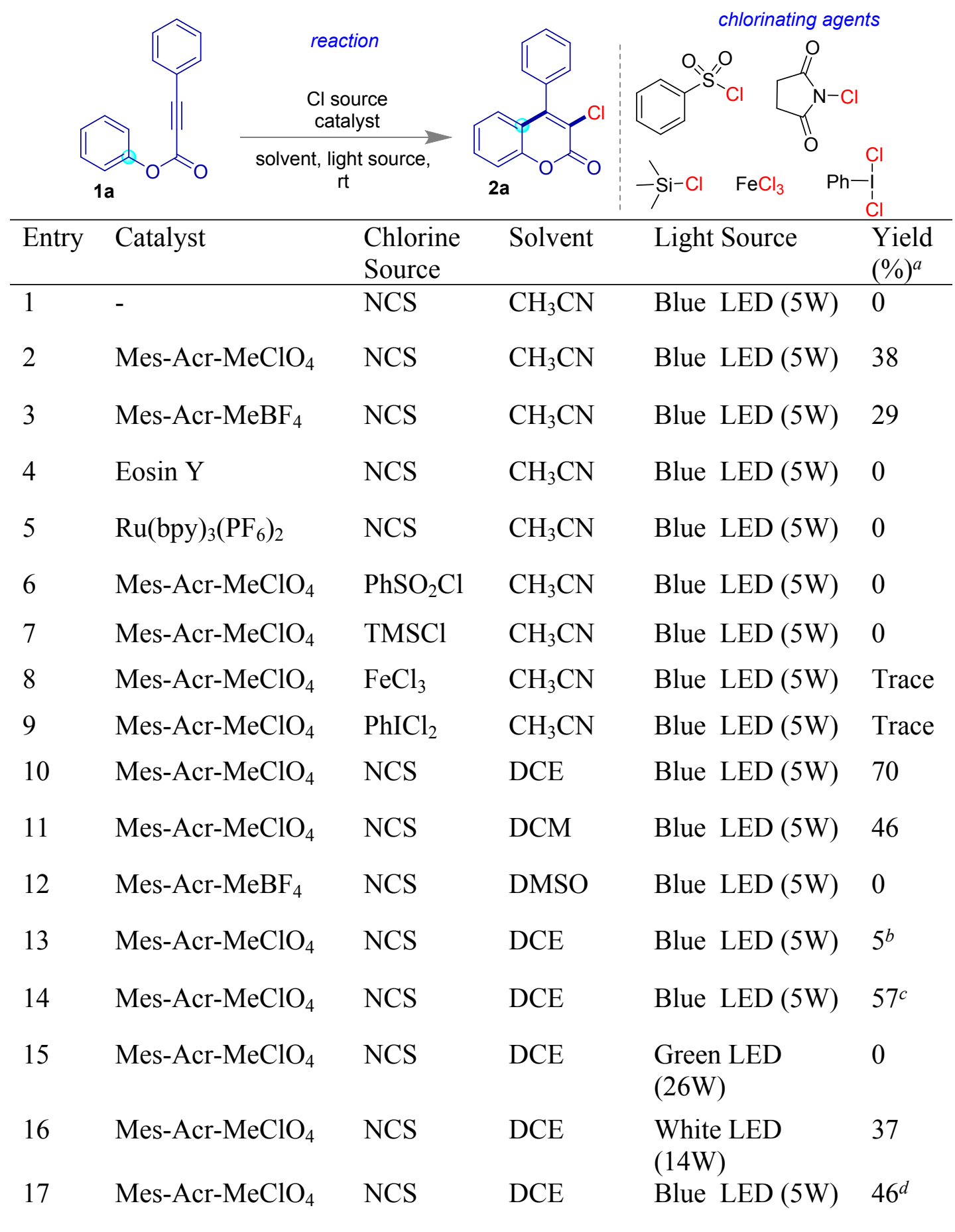

${ }^{a}$ Reaction Condition: 1a (0.270 mmol, $\left.60 \mathrm{mg}\right), \mathrm{N}$-chlorosuccinimide $(0.810 \mathrm{mmol}, 108 \mathrm{mg})$, Mes-Acr- $\mathrm{MeClO}_{4}(5 \mathrm{~mol} \%, 0.0135 \mathrm{mmol}, 6 \mathrm{mg})$ in $1.5 \mathrm{~mL}$ dry solvent at inert atmosphere for $24 \mathrm{~h}$ using visible light, ${ }^{b} \mathrm{O}_{2}$ atmosphere, ${ }^{c} 2.0$ equiv NCS, ${ }^{d}$ after $12 \mathrm{~h}$. 


\section{CHARATERIZATION DATA}

3-Chloro-4-phenyl-2H-chromen-2-one (2a): $\mathrm{R}_{\mathrm{f}}=0.4$ (5\% ethyl acetate in hexane); white solid;

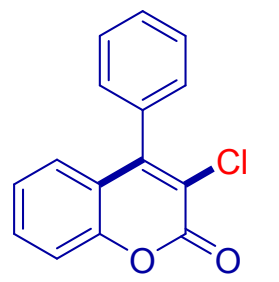
yield 70\% (48 mg); mp 139-143 ${ }^{\circ} \mathrm{C} ;{ }^{1} \mathrm{H}$ NMR (400 MHz, $\left.\mathrm{CDCl}_{3}\right) \delta$ 7.60-7.52 (m, 4H), $7.42(\mathrm{dd}, J=8.2,0.6 \mathrm{~Hz}, 1 \mathrm{H}), 7.35-7.32(\mathrm{~m}, 2 \mathrm{H}), 7.23-7.19(\mathrm{~m}, 1 \mathrm{H})$, $7.11(\mathrm{dd}, J=8.0,1.5 \mathrm{~Hz}, 1 \mathrm{H}) ;{ }^{13} \mathrm{C} \mathrm{NMR}\left(100 \mathrm{MHz}, \mathrm{CDCl}_{3}\right) \delta 157.7,152.1$, 151.3, 133.2, 132.0, 129.6, 129.0, 128.5, 127.6, 124.9, 120.7, 120.3, 117.0; IR (KBr) v 2923, 1732, 1600, 1010, 754; HRMS (ESI/Q-TOF) m/z: $[\mathrm{M}+\mathrm{H}]^{+}$calcd for $\mathrm{C}_{15} \mathrm{H}_{10} \mathrm{ClO}_{2}$ 257.0364; found 257.0362 .

7-Bromo-3-chloro-4-phenyl-2H-chromen-2-one (2b): $\mathrm{R}_{\mathrm{f}}=0.45$ (5\% ethyl acetate in hexane);<smiles>O=c1oc2cc(Br)ccc2c(-c2ccccc2)c1Cl</smiles>
white solid; yield $76 \%$ (51 mg); mp 190-192 ${ }^{\circ} \mathrm{C} ;{ }^{1} \mathrm{H}$ NMR (400 MHz, $\left.\mathrm{CDCl}_{3}\right) \delta$ 7.60-7.53 (m, 3H), $7.43(\mathrm{~d}, J=2.0 \mathrm{~Hz}, 1 \mathrm{H}), 7.32-7.30(\mathrm{~m}, 2 \mathrm{H})$, $7.18(\mathrm{dd}, J=8.6,2.0 \mathrm{~Hz}, 1 \mathrm{H}), 7.04(\mathrm{~d}, J=8.6 \mathrm{~Hz}, 1 \mathrm{H}) ;{ }^{13} \mathrm{C}$ NMR $(100$ $\left.\mathrm{MHz}, \mathrm{CDCl}_{3}\right) \delta 157.0,152.3,150.7,137.9,132.9,129.8,129.2,128.5(\times 2), 125.5,120.8,119.0$, 117.3; IR (KBr) $\bar{v} 3024,1737,1595,1013,766$; HRMS (ESI/Q-TOF) m/z: $[\mathrm{M}+\mathrm{H}]^{+}$calcd for $\mathrm{C}_{15} \mathrm{H}_{9} \mathrm{BrClO}_{2}$ 334.9469; found 334.9480.

3,7-Dichloro-4-phenyl-2H-chromen-2-one (2c): $\mathrm{R}_{\mathrm{f}}=0.5$ (10\% ethyl acetate in hexane); white<smiles>O=c1oc2cc(Cl)ccc2c(-c2ccccc2)c1Cl</smiles>
solid; yield 78\% (53 mg); mp 186-190 ${ }^{\circ} \mathrm{C} ;{ }^{1} \mathrm{H}$ NMR (400 MHz, $\left.\mathrm{CDCl}_{3}\right) \delta$ 7.60-7.52 (m, 3H), $7.42(\mathrm{~d}, J=2.0 \mathrm{~Hz}, 1 \mathrm{H}), 7.32-7.30(\mathrm{~m}, 2 \mathrm{H}), 7.18(\mathrm{dd}, J$ $=8.6,2.0 \mathrm{~Hz}, 1 \mathrm{H}), 7.04(\mathrm{~d}, J=8.6 \mathrm{~Hz}, 1 \mathrm{H}) ;{ }^{13} \mathrm{C} \mathrm{NMR}\left(100 \mathrm{MHz}, \mathrm{CDCl}_{3}\right)$ $\delta 157.0,152.3,150.7,137.9,132.8,129.8,129.1,128.45,128.43,125.5,120.7,118.9,117.2 ;$ IR $(\mathrm{KBr}) \bar{v}$ 3029, 1739, 1597, 1014, 766; HRMS (ESI/Q-TOF) m/z: $[\mathrm{M}+\mathrm{Na}]^{+}$calcd for $\mathrm{C}_{15} \mathrm{H}_{8} \mathrm{Cl}_{2} \mathrm{O}_{2} \mathrm{Na} 312.9794$; found 312.9787 . 
3-Chloro-7-fluoro-4-phenyl-2H-chromen-2-one (2d): $\mathrm{R}_{\mathrm{f}}=0.45$ (10\% ethyl acetate in hexane);

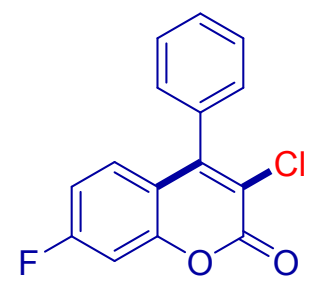
white solid; yield 83\% (57 mg); mp 183-184 ${ }^{\circ} \mathrm{C}$; ${ }^{1} \mathrm{H}$ NMR (400 MHz, $\left.\mathrm{CDCl}_{3}\right) \delta 7.60-7.52(\mathrm{~m}, 3 \mathrm{H}), 7.33-7.31(\mathrm{~m}, 2 \mathrm{H}), 7.15-7.08(\mathrm{~m}, 2 \mathrm{H}), 6.97-$ $6.92(\mathrm{~m}, 1 \mathrm{H}) ;{ }^{13} \mathrm{C} \mathrm{NMR}\left(100 \mathrm{MHz}, \mathrm{CDCl}_{3}\right) \delta 164.4\left(\mathrm{~d},{ }^{1} J_{C-F}=255 \mathrm{~Hz}\right)$, $157.3,153.2\left(\mathrm{~d},{ }^{3} J_{C-F}=13 \mathrm{~Hz}\right), 150.9,133.1,129.8,129.3\left(\mathrm{~d},{ }^{3} J_{C-F}=10 \mathrm{~Hz}\right), 129.1,128.4,119.7$ $\left(\mathrm{d},{ }^{4} J_{C-F}=3.4 \mathrm{~Hz}\right), 117.1\left(\mathrm{~d},{ }^{4} J_{C-F}=2.8 \mathrm{~Hz}\right), 113.0\left(\mathrm{~d},{ }^{2} J_{C-F}=22.6 \mathrm{~Hz}\right), 104.6\left(\mathrm{~d},{ }^{2} J_{C-F}=26 \mathrm{~Hz}\right)$; IR $(\mathrm{KBr}) \bar{v}$ 2956, 1736, 1020, 772; HRMS (ESI/Q-TOF) m/z: $[\mathrm{M}+\mathrm{Na}]^{+}$calcd for $\mathrm{C}_{15} \mathrm{H}_{8} \mathrm{ClFO}_{2} \mathrm{Na} 297.0089$; found 297.0069.

3-Chloro-4-phenyl-7-(trifluoromethyl)-2H-chromen-2-one (2e): $\mathrm{R}_{\mathrm{f}}=0.4(10 \%$ ethyl acetate<smiles>O=c1oc2cc(C(F)(F)F)ccc2c(-c2ccccc2)c1Cl</smiles>
in hexane); white solid; yield 78\% (52 mg); mp 140-142 ${ }^{\circ} \mathrm{C}$; ${ }^{1} \mathrm{H}$ NMR $\left(700 \mathrm{MHz}, \mathrm{CDCl}_{3}\right) \delta 7.67(\mathrm{~s}, 1 \mathrm{H}), 7.61-7.56(\mathrm{~m}, 3 \mathrm{H}), 7.44(\mathrm{~d}, J=8.4 \mathrm{~Hz}$, 1H), 7.34-7.32 (m, 2H), $7.25(\mathrm{~d}, J=8.4 \mathrm{~Hz}, 1 \mathrm{H}) ;{ }^{13} \mathrm{C} \mathrm{NMR}(175 \mathrm{MHz}$, $\left.\mathrm{CDCl}_{3}\right) \delta 156.7,151.7,150.2,133.54\left(\mathrm{q},{ }^{2} J_{C F 3}=33.7 \mathrm{~Hz}\right), 132.5,130.0$ 129.3, 128.44, 128.43, $123.15\left(\mathrm{q},{ }^{1} J_{C F 3}=272.8 \mathrm{~Hz}\right), 123.1,122.9,121.4\left(\mathrm{q},{ }^{4} J_{C F 3}=3.4 \mathrm{~Hz}\right)$, $114.5\left(\mathrm{q},{ }^{4} J_{C F 3}=3.4 \mathrm{~Hz}\right)$; IR (KBr) $\bar{v} 3011,1743,1603,1329,1133,1017,748$; HRMS (ESI/QTOF) $\mathrm{m} / \mathrm{z}$ : $[\mathrm{M}+\mathrm{H}]^{+}$calcd for $\mathrm{C}_{16} \mathrm{H}_{9} \mathrm{ClF}_{3} \mathrm{O}_{2}$ 325.0238; found 325.0238.

3-Chloro-4-phenyl-6-(trifluoromethyl)-2H-chromen-2-one (2f): $R_{\mathrm{f}}=0.4(10 \%$ ethyl acetate in<smiles>O=c1oc2ccc(C(F)(F)F)cc2c(-c2ccccc2)c1Cl</smiles>
hexane); colorless liquid; yield 72\% (48 mg); ${ }^{1} \mathrm{H}$ NMR (700 MHz, $\left.\mathrm{CDCl}_{3}\right) \delta 7.79(\mathrm{dd}, J=8.7,1.7 \mathrm{~Hz}, 1 \mathrm{H}), 7.62-7.58(\mathrm{~m}, 3 \mathrm{H}), 7.53(\mathrm{~d}, J=$ $8.7 \mathrm{~Hz}, 1 \mathrm{H}), 7.37(\mathrm{~s}, 1 \mathrm{H}), 7.34-7.33(\mathrm{~m}, 2 \mathrm{H}) ;{ }^{13} \mathrm{C} \mathrm{NMR}(175 \mathrm{MHz}$, 
$\left.\mathrm{CDCl}_{3}\right) \delta 156.7,153.8,150.5,132.3,130.2,129.4,128.6\left(\mathrm{q},{ }^{4} J_{C F 3}=4.1 \mathrm{~Hz}\right), 128.4,127.5(\mathrm{q}$, $\left.{ }^{2} J_{C F 3}=33.5 \mathrm{~Hz}\right), 124.9\left(\mathrm{q},{ }^{4} J_{C F 3}=4.1 \mathrm{~Hz}\right), 123.41\left(\mathrm{q},{ }^{1} J_{C F 3}=272.4 \mathrm{~Hz}\right), 122.4,120.5,117.9 ;$ IR $(\mathrm{KBr}) \bar{v}$ 3054, 1743, 1306, 1009, 770; HRMS (ESI/Q-TOF) m/z: $[\mathrm{M}+\mathrm{H}]^{+}$calcd for $\mathrm{C}_{16} \mathrm{H}_{9} \mathrm{ClF}_{3} \mathrm{O}_{2}$ 325.0238; found 325.0254.

3-Chloro-4-phenyl-7-(trifluoromethoxy)-2H-chromen-2-one (2g): $\mathrm{R}_{\mathrm{f}}=0.35$ (10\% ethyl acetate in hexane); semi solid; yield 66\% (44 mg); ${ }^{1} \mathrm{H} \mathrm{NMR}(700 \mathrm{MHz}$,
$\left.\mathrm{CDCl}_{3}\right) \delta 7.59-7.54(\mathrm{~m}, 3 \mathrm{H}), 7.33-7.32(\mathrm{~m}, 2 \mathrm{H}), 7.29(\mathrm{~s}, 1 \mathrm{H}), 7.15(\mathrm{~d}, J$ 157.0, 152.6, 151.3, 150.5, 132.8, 129.9, 129.2, 129.0, 128.4, 120.9, $120.3\left(\mathrm{q},{ }^{1} J_{C F 3}=259.8 \mathrm{~Hz}\right)$, 118.8, 117.2, 109.2; IR (KBr) $\bar{v} 3012,1742,1608,1025,770$; HRMS (ESI/Q-TOF) m/z: [M + $\mathrm{H}]^{+}$calcd for $\mathrm{C}_{16} \mathrm{H}_{9} \mathrm{ClF}_{3} \mathrm{O}_{3} 341.0187$; found 341.0188.

3-Chloro-2-oxo-4-phenyl-2H-chromene-7-carbonitrile (2h): $\mathrm{R}_{\mathrm{f}}=0.3(10 \%$ ethyl acetate in<smiles>N#Cc1ccc2c(-c3ccccc3)c(Cl)c(=O)oc2c1</smiles>
hexane); pale yellow solid; yield $80 \%$ (55 mg); mp $194-196{ }^{\circ} \mathrm{C} ;{ }^{1} \mathrm{H}$ NMR $\left(400 \mathrm{MHz}, \mathrm{CDCl}_{3}\right) \delta 7.69(\mathrm{~d}, J=1.6 \mathrm{~Hz}, 1 \mathrm{H}), 7.62-7.55(\mathrm{~m}, 3 \mathrm{H}), 7.46(\mathrm{dd}$, $J=8.4,1.6 \mathrm{~Hz}, 1 \mathrm{H}), 7.32-7.29(\mathrm{~m}, 2 \mathrm{H}), 7.23(\mathrm{~d}, J=8.4 \mathrm{~Hz}, 1 \mathrm{H}) ;{ }^{13} \mathrm{C}$ NMR $\left(100 \mathrm{MHz}, \mathrm{CDCl}_{3}\right) \delta 156.2,151.5,149.9,132.1,130.2,129.4$, 128.5, 128.4, 127.9, 123.9, 123.8, 120.8, 117.3, 114.9; IR (KBr) v 3021, 2922, 2233, 1744, 761; HRMS (ESI/Q-TOF) m/z: [M + Na] calcd for $\mathrm{C}_{16} \mathrm{H}_{8} \mathrm{ClNO}_{2} \mathrm{Na}$ 304.0136; found 304.0139.

3-Chloro-2-oxo-4-phenyl-2H-chromene-6-carbonitrile (2i): $\mathrm{R}_{\mathrm{f}}=0.35(10 \%$ ethyl acetate in hexane); yellow solid; yield $69 \%(47 \mathrm{mg}) ; \mathrm{mp} 158-160{ }^{\circ} \mathrm{C} ;{ }^{1} \mathrm{H}$ NMR (400 
$\left.\mathrm{MHz}, \mathrm{CDCl}_{3}\right) \delta 7.79(\mathrm{dd}, J=8.6,2.0 \mathrm{~Hz}, 1 \mathrm{H}), 7.63-7.59(\mathrm{~m}, 3 \mathrm{H}), 7.51(\mathrm{~d}, J=8.6 \mathrm{~Hz}, 1 \mathrm{H}), 7.43$ $(\mathrm{d}, J=2.0 \mathrm{~Hz}, 1 \mathrm{H}), 7.33-7.30(\mathrm{~m}, 2 \mathrm{H}) ;{ }^{13} \mathrm{C} \mathrm{NMR}\left(175 \mathrm{MHz}, \mathrm{CDCl}_{3}\right) \delta 156.2,154.2,149.8$ 134.6, 132.2, 131.9, 130.3, 129.5, 128.3, 122.9, 121.2, 118.5, 117.6, 109.2; IR (KBr) v̄ 3022, 2234, 1745, 1606, 759; HRMS (ESI/Q-TOF) m/z: $[\mathrm{M}+\mathrm{Na}]^{+}$calcd for $\mathrm{C}_{16} \mathrm{H}_{8} \mathrm{NClO}_{2} \mathrm{Na}$ 304.0136; found 304.0141.

3-Chloro-7-nitro-4-phenyl-2H-chromen-2-one $\mathbf{( 2 j}): \mathrm{R}_{\mathrm{f}}=0.5$ (15\% ethyl acetate in hexane); white solid; yield $89 \%(60 \mathrm{mg}) ; \mathrm{mp} 182-185{ }^{\circ} \mathrm{C} ;{ }^{1} \mathrm{H}$ NMR $(400 \mathrm{MHz}$,
$\left.\mathrm{CDCl}_{3}\right) \delta 8.25(\mathrm{~d}, J=2.2 \mathrm{~Hz}, 1 \mathrm{H}), 8.04(\mathrm{dd}, J=8.8,2.2 \mathrm{~Hz}, 1 \mathrm{H}), 7.64-$ $\left.\mathrm{MHz}, \mathrm{CDCl}_{3}\right) \delta 156.3,151.7,149.7,149.1,132.2,130.2,129.4,128.7,128.4,125.1,124.4$, 119.4, 112.7; IR (KBr) v 2915, 1743, 1344, 1020, 770; HRMS (ESI/Q-TOF) m/z: [M + Na $]^{+}$ calcd for $\mathrm{C}_{15} \mathrm{H}_{8} \mathrm{NClO}_{4} \mathrm{Na}$ 324.0034; found 324.0043.

3-Chloro-6-nitro-4-phenyl-2H-chromen-2-one (2k): $\mathrm{R}_{\mathrm{f}}=0.55$ (15\% ethyl acetate in hexane); yellow solid; yield $77 \%(52 \mathrm{mg}) ; \mathrm{mp} 159-162{ }^{\circ} \mathrm{C} ;{ }^{1} \mathrm{H} \mathrm{NMR}(400 \mathrm{MHz}$,
$\left.\mathrm{CDCl}_{3}\right) \delta 8.40(\mathrm{dd}, J=9.0,2.6 \mathrm{~Hz}, 1 \mathrm{H}), 8.03(\mathrm{~d}, J=2.6 \mathrm{~Hz}, 1 \mathrm{H}), 7.66-$ $\left.\mathrm{MHz}, \mathrm{CDCl}_{3}\right) \delta 156.1,155.4,150.1,144.6,131.8,130.5,129.5,128.4,126.6,123.5,123.1$, 120.7, 118.3; IR (KBr) v 2923, 1746, 1600, 1345, 758; HRMS (ESI/Q-TOF) m/z: [M + Na $]^{+}$ calcd for $\mathrm{C}_{15} \mathrm{H}_{8} \mathrm{ClNO}_{4} \mathrm{Na}$ 324.0034; found 324.0036. 
3-Chloro-4-phenyl-2H-benzo[g]chromen-2-one (21): $\mathrm{R}_{\mathrm{f}}=0.3(10 \%$ ethyl acetate in hexane);

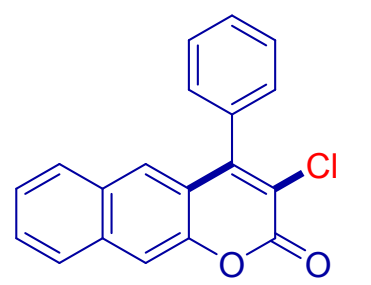
white solid; yield 71\% (48 mg); mp 183-185 ${ }^{\circ} \mathrm{C} ;{ }^{1} \mathrm{H}$ NMR (400 MHz, $\left.\mathrm{CDCl}_{3}\right) \delta 8.62-8.60(\mathrm{~m}, 1 \mathrm{H}), 7.87-7.85(\mathrm{~m}, 1 \mathrm{H}), 7.70-7.65(\mathrm{~m}, 2 \mathrm{H}), 7.62-$ $7.54(\mathrm{~m}, 4 \mathrm{H}), 7.39-7.36(\mathrm{~m}, 2 \mathrm{H}), 7.09(\mathrm{~d}, J=8.8 \mathrm{~Hz}, 1 \mathrm{H}) ;{ }^{13} \mathrm{C}$ NMR $(100$ $\left.\mathrm{MHz}, \mathrm{CDCl}_{3}\right) \delta 157.9,152.3,149.3,134.8,133.7,129.6,129.2,129.0,128.6,127.9,127.7$, 124.8, 123.0, 122.8, 122.6, 120.4, 115.5; IR (KBr) v 3033, 1732, 1060, 754; HRMS (ESI/QTOF) $\mathrm{m} / \mathrm{z}:[\mathrm{M}+\mathrm{Na}]^{+}$calcd for $\mathrm{C}_{19} \mathrm{H}_{11} \mathrm{ClO}_{2} \mathrm{Na} 329.0340$; found 329.0345.

3-Chloro-4,7-diphenyl-2H-chromen-2-one (2m): $\mathrm{R}_{\mathrm{f}}=0.35$ (5\% ethyl acetate in hexane);<smiles>O=c1oc2cc(-c3ccccc3)ccc2c(-c2ccccc2)c1Cl</smiles>
white solid; yield 66\% (44 mg); mp 188-190 ${ }^{\circ} \mathrm{C}$; ${ }^{1} \mathrm{H}$ NMR (400 MHz, $\left.\mathrm{CDCl}_{3}\right) \delta$ 7.63-7.55 (m, 6H), 7.50-7.42 (m, 4H), 7.38-7.35 (m, 2H), 7.17 $(\mathrm{d}, J=8.4 \mathrm{~Hz}, 1 \mathrm{H}) ;{ }^{13} \mathrm{C} \mathrm{NMR}\left(100 \mathrm{MHz}, \mathrm{CDCl}_{3}\right) \delta 157.7,152.5,151.1$, $145.1,138.9,133.3,129.6,129.3,129.0,128.8,128.5,127.9,127.3,123.7,120.3,119.2,115.0$; IR (KBr) v 3044, 2903, 1775, 1602, 750; HRMS (ESI/Q-TOF) m/z: [M + Na $]^{+}$calcd for $\mathrm{C}_{21} \mathrm{H}_{13} \mathrm{ClO}_{2} \mathrm{Na} 355.0496$; found 355.0508.

3-Chloro-4-phenyl-7-trityl-2H-chromen-2-one (2n): $\mathrm{R}_{\mathrm{f}}=0.4$ (5\% ethyl acetate in hexane);<smiles>O=c1oc2cc(C(c3ccccc3)(c3ccccc3)c3ccccc3)ccc2c(-c2ccccc2)c1Cl</smiles>
white solid; yield 76\% (49 mg); mp $>240{ }^{\circ} \mathrm{C}$; ${ }^{1} \mathrm{H}$ NMR (400 MHz, $\left.\mathrm{CDCl}_{3}\right) \delta 7.56-7.50(\mathrm{~m}, 3 \mathrm{H}), 7.39-7.38(\mathrm{~m}, 1 \mathrm{H}), 7.34-7.32(\mathrm{~m}, 2 \mathrm{H})$ 7.28-7.25 (m, 6H), 7.23-7.18 (m, 9H), 7.07-7.05 (m, 1H), $6.98(\mathrm{~d}, J=$ 8.6 Hz, 1H); ${ }^{13} \mathrm{C}$ NMR (100 MHz, $\left.\mathrm{CDCl}_{3}\right) \delta 157.8,152.2,151.6,151.0$, $145.7,133.2,131.0,129.6,128.9,128.55,128.53,128.0,126.5,126.1,120.3,119.0,118.1,65.2$; 
IR (KBr) v 3023, 2928, 1731, 1031, 755; HRMS (ESI/Q-TOF) m/z: $[\mathrm{M}+\mathrm{H}]^{+}$calcd for $\mathrm{C}_{34} \mathrm{H}_{24} \mathrm{ClO}_{2} 499.1459$; found 499.1445 .

3-Chloro-7-methyl-4-phenyl-2H-chromen-2-one (2o): $\mathrm{R}_{\mathrm{f}}=0.45$ (5\% ethyl acetate in hexane);

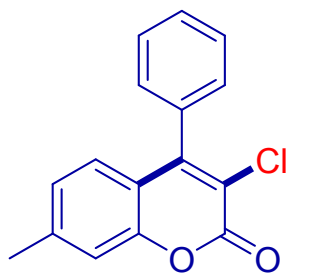
white solid; yield 48\% (33 mg); mp 177-180 ${ }^{\circ} \mathrm{C}$; ${ }^{1} \mathrm{H} \mathrm{NMR}$ (400 MHz, $\mathrm{CDCl}_{3}$ ) $\delta$ 7.58-7.51 (m, 3H), 7.33-7.31 (m, 2H), 7.22 (s, 1H), 7.03-6.97 (m, 2H), 2.45 (s, 3H); ${ }^{13} \mathrm{C}$ NMR $\left(100 \mathrm{MHz}, \mathrm{CDCl}_{3}\right) \delta 157.9,152.2,151.3,143.4,133.4$, 129.5, 128.9, 128.5, 127.3, 126.1, 119.5, 117.9, 117.1, 21.8; IR (KBr) v 2921, 1731, 1595, 1036, 746; HRMS (ESI/Q-TOF) m/z: [M + Na] calcd for $\mathrm{C}_{16} \mathrm{H}_{11} \mathrm{ClO}_{2} \mathrm{Na} 293.0340$; found 293.0349.

7-(tert-Butyl)-3-chloro-4-phenyl-2H-chromen-2-one (2p): $\mathrm{R}_{\mathrm{f}}=0.55(5 \%$ ethyl acetate in<smiles></smiles>
hexane); white solid; yield $42 \%$ (28 mg); mp 130-133 ${ }^{\circ} \mathrm{C}$; ${ }^{1} \mathrm{H}$ NMR (400 $\left.\mathrm{MHz}, \mathrm{CDCl}_{3}\right) \delta$ 7.58-7.53 (m, 3H), $7.41(\mathrm{~d}, J=1.8 \mathrm{~Hz}, 1 \mathrm{H}), 7.34-7.31(\mathrm{~m}$, 2H), $7.24(\mathrm{dd}, J=8.4,1.8 \mathrm{~Hz}, 1 \mathrm{H}), 7.03(\mathrm{~d}, J=8.4 \mathrm{~Hz}, 1 \mathrm{H}), 1.34$ (s, 9H); ${ }^{13} \mathrm{C}$ NMR $\left(100 \mathrm{MHz}, \mathrm{CDCl}_{3}\right) \delta 158.0,156.6,152.2,151.2,133.4,129.5$, 128.9, 128.5, 127.1, 122.4, 119.7, 117.8, 113.8, 35.4, 31.1; IR (KBr) v̄ 2989, 1764, 1655, 745; HRMS (ESI/Q-TOF) m/z: [M + H] calcd for $\mathrm{C}_{19} \mathrm{H}_{18} \mathrm{ClO}_{2}$ 313.0990; found 313.1019.

3-Chloro-7-(1-chloroethyl)-4-phenyl-2H-chromen-2-one (2q): $\mathrm{R}_{\mathrm{f}}=0.5(10 \%$ ethyl acetate in<smiles>CC(Cl)c1ccc2c(-c3ccccc3)c(Cl)c(=O)oc2c1</smiles>
hexane); colorless liquid; yield 64\% (49 mg); mp 154-155 ${ }^{\circ} \mathrm{C}$; ${ }^{1} \mathrm{H}$ NMR $\left(400 \mathrm{MHz}, \mathrm{CDCl}_{3}\right) \delta$ 7.60-7.52 (m, 3H), $7.46(\mathrm{~d}, J=1.8 \mathrm{~Hz}, 1 \mathrm{H}), 7.33-$ $7.31(\mathrm{~m}, 2 \mathrm{H}), 7.28-7.25(\mathrm{~m}, 1 \mathrm{H}), 7.10(\mathrm{~d}, J=8.4 \mathrm{~Hz}, 1 \mathrm{H}), 5.11(\mathrm{q}, J=6.8$ $\mathrm{Hz}, 1 \mathrm{H}), 1.86(\mathrm{~d}, J=6.8 \mathrm{~Hz}, 3 \mathrm{H}) ;{ }^{13} \mathrm{C} \mathrm{NMR}\left(100 \mathrm{MHz}, \mathrm{CDCl}_{3}\right) \delta 157.5$, 
$152.1,150.8,147.2,133.1,129.7,129.1,128.5,128.0,123.2,121.0,120.0,115.0,57.2,26.4$; IR $(\mathrm{KBr}) \bar{v}$ 3050, 1734, 1022, 744; HRMS (ESI/Q-TOF) m/z: $[\mathrm{M}+\mathrm{H}]^{+}$calcd for $\mathrm{C}_{17} \mathrm{H}_{13} \mathrm{Cl}_{2} \mathrm{O}_{2}$ 319.0287; found 319.0304 .

3-Chloro-6-methoxy-4-phenyl-2H-chromen-2-one (2s): $\mathrm{R}_{\mathrm{f}}=0.3(10 \%$ ethyl acetate in<smiles>COc1ccc2oc(=O)c(Cl)c(-c3ccccc3)c2c1</smiles>
hexane); white solid; yield 47\% (32 mg); mp 179-181 ${ }^{\circ} \mathrm{C}$; ${ }^{1} \mathrm{H}$ NMR (700 $\left.\mathrm{MHz}, \mathrm{CDCl}_{3}\right) \delta 7.57(\mathrm{t}, J=7.4 \mathrm{~Hz}, 2 \mathrm{H}), 7.55-7.53(\mathrm{~m}, 1 \mathrm{H}), 7.35-7.32(\mathrm{~m}$, $3 \mathrm{H}), 7.11(\mathrm{dd}, J=9.0,2.7 \mathrm{~Hz}, 1 \mathrm{H}), 6.52(\mathrm{~d}, J=2.7 \mathrm{~Hz}, 1 \mathrm{H}), 3.68(\mathrm{~s}, 3 \mathrm{H})$; ${ }^{13} \mathrm{C}$ NMR $\left(175 \mathrm{MHz}, \mathrm{CDCl}_{3}\right) \delta 157.8,156.4,151.0,146.6,133.3,129.6,129.1,128.4,121.3$, 120.8, 119.0, 118.0, 110.5, 55.9; IR (KBr) v 3066, 2839, 1745, 1541, 1052, 760; HRMS (ESI/QTOF) m/z: $[\mathrm{M}+\mathrm{Na}]^{+}$calcd for $\mathrm{C}_{16} \mathrm{H}_{11} \mathrm{ClO}_{3} \mathrm{Na}$ 309.0289; found 309.0303 .

3,6-Dichloro-4-phenyl-2H-chromen-2-one (2t): $\mathrm{R}_{\mathrm{f}}=0.35$ ( $10 \%$ ethyl acetate in hexane); semi<smiles>O=c1oc2ccc(Cl)cc2c(-c2ccccc2)c1Cl</smiles>
solid; yield 56\% (38 mg); ${ }^{1} \mathrm{H}$ NMR $\left(700 \mathrm{MHz}, \mathrm{CDCl}_{3}\right) \delta$ 7.60-7.54 (m, 3H), $7.49(\mathrm{dd}, J=8.7,1.5 \mathrm{~Hz}, 1 \mathrm{H}), 7.36(\mathrm{~d}, J=8.7 \mathrm{~Hz}, 1 \mathrm{H}), 7.32(\mathrm{~d}, J=7.5 \mathrm{~Hz}$, 2H), $7.06(\mathrm{~d}, J=1.5 \mathrm{~Hz}, 1 \mathrm{H}) ;{ }^{13} \mathrm{C} \mathrm{NMR}\left(175 \mathrm{MHz}, \mathrm{CDCl}_{3}\right) \delta 157.0,150.5$, 150.2, 132.6, 131.9, 130.5, 129.9, 129.3, 128.4, 126.8, 122.1, 121.4, 118.5; IR (KBr) v 3032, 1758, 1587, 1003, 786; HRMS (ESI/Q-TOF) m/z: [M + Na $]^{+}$calcd for $\mathrm{C}_{15} \mathrm{H}_{8} \mathrm{Cl}_{2} \mathrm{O}_{2} \mathrm{Na} 312.9794$; found 312.9804 .

3-Chloro-4-(4-chlorophenyl)-2H-chromen-2-one (4a): $\mathrm{R}_{\mathrm{f}}=0.45(10 \%$ ethyl acetate in<smiles>O=c1oc2ccccc2c(-c2ccc(Cl)cc2)c1Cl</smiles>
hexane); semi solid; yield 65\% (44 mg); ${ }^{1} \mathrm{H}$ NMR $\left(700 \mathrm{MHz}, \mathrm{CDCl}_{3}\right) \delta$ 7.56$7.55(\mathrm{~m}, 3 \mathrm{H}), 7.42(\mathrm{~d}, J=8.3 \mathrm{~Hz}, 1 \mathrm{H}), 7.29(\mathrm{~d}, J=8.3 \mathrm{~Hz}, 2 \mathrm{H}), 7.23$ (t, $J=7.5$ 
$\mathrm{Hz}, 1 \mathrm{H}), 7.09(\mathrm{dd}, J=8.1,1.1 \mathrm{~Hz}, 1 \mathrm{H}) ;{ }^{13} \mathrm{C} \mathrm{NMR}\left(175 \mathrm{MHz}, \mathrm{CDCl}_{3}\right) \delta 157.4,152.2,150.1$, 135.9, 132.2, 131.6, 130.1, 129.5, 127.3, 125.0, 121.0, 120.0, 117.2; IR (KBr) v 3085, 1782, 1578, 1042, 782; HRMS (ESI/Q-TOF) m/z: [M + Na $]^{+}$calcd for $\mathrm{C}_{15} \mathrm{H}_{8} \mathrm{Cl}_{2} \mathrm{O}_{2} \mathrm{Na}$ 312.9794; found 312.9782.

3-Chloro-4-(4-fluorophenyl)-2H-chromen-2-one (4b): $\mathrm{R}_{\mathrm{f}}=0.45(10 \%$ ethyl acetate in<smiles>O=c1oc2ccccc2c(-c2ccc(F)cc2)c1Cl</smiles>
hexane); white solid; yield 65\% (45 mg); mp 127-130 ${ }^{\circ} \mathrm{C}$; ${ }^{1} \mathrm{H}$ NMR (700 MHz, $\left.\mathrm{CDCl}_{3}\right) \delta 7.56(\mathrm{t}, J=7.7 \mathrm{~Hz}, 1 \mathrm{H}), 7.42(\mathrm{~d}, J=8.3 \mathrm{~Hz}, 1 \mathrm{H}), 7.35-7.33(\mathrm{~m}, 2 \mathrm{H})$, 7.28-7.26 (m, 2H), $7.23(\mathrm{t}, J=7.7 \mathrm{~Hz}, 1 \mathrm{H}), 7.10(\mathrm{~d}, J=8.0 \mathrm{~Hz}, 1 \mathrm{H}) ;{ }^{13} \mathrm{C} \mathrm{NMR}$ $\left(175 \mathrm{MHz}, \mathrm{CDCl}_{3}\right) \delta 163.3\left(\mathrm{~d},{ }^{1} J_{\mathrm{C}-F}=250.2 \mathrm{~Hz}\right), 157.5,152.1,150.3,132.1$, $130.7\left(\mathrm{~d},{ }^{3} J_{C-F}=8.4 \mathrm{~Hz}\right), 129.1\left(\mathrm{~d},{ }^{4} J_{C-F}=3.6 \mathrm{~Hz}\right), 127.3,125.0,121.1,120.2,117.1,116.3(\mathrm{~d}$, ${ }^{2} J_{C-F}=22.0 \mathrm{~Hz}$ ); IR (KBr) $\bar{v} 3003,1722,1604,1012,744 ;$ HRMS (ESI/Q-TOF) m/z: [M + H] ${ }^{+}$ calcd for $\mathrm{C}_{15} \mathrm{H}_{9} \mathrm{ClFO}_{2} 275.0270$; found 275.0268 .

3-Chloro-4-(2,4-difluorophenyl)-2H-chromen-2-one (4c): $\mathrm{R}_{\mathrm{f}}=0.6(10 \%$ ethyl acetate in<smiles>O=c1oc2ccccc2c(-c2ccc(F)cc2F)c1Cl</smiles>
hexane); white solid; yield $82 \%$ (56 mg); mp $138-140{ }^{\circ} \mathrm{C}$; ${ }^{1} \mathrm{H}$ NMR (700 MHz, $\left.\mathrm{CDCl}_{3}\right) \delta$ 7.58-7.56 (m, 1H), $7.43(\mathrm{~d}, J=8.3 \mathrm{~Hz}, 1 \mathrm{H}), 7.31-7.28(\mathrm{~m}, 1 \mathrm{H}), 7.26-$ $7.23(\mathrm{~m}, 1 \mathrm{H}), 7.11(\mathrm{t}, J=6.9 \mathrm{~Hz}, 1 \mathrm{H}), 7.06-7.04(\mathrm{~m}, 2 \mathrm{H}) ;{ }^{13} \mathrm{C} \mathrm{NMR}(175 \mathrm{MHz}$, $\left.\mathrm{CDCl}_{3}\right) \delta 164.8\left(\mathrm{~d},{ }^{3} J_{C F}=11.5 \mathrm{~Hz}\right), 163.34\left(\mathrm{~d},{ }^{3} J_{C F}=11.5 \mathrm{~Hz}\right), 160.2\left(\mathrm{~d},{ }^{3} J_{C-F}=\right.$ $12.1 \mathrm{~Hz}), 158.78\left(\mathrm{~d},{ }^{3} J_{C-F}=12.1 \mathrm{~Hz}\right), 157.1,152.0,145.2,132.3,131.43\left(\mathrm{~d},{ }^{4} J_{C-F}=4.3 \mathrm{~Hz}\right)$, $131.38\left(\mathrm{~d},{ }^{4} J_{C-F}=4.3 \mathrm{~Hz}\right), 125.9\left(\mathrm{~d},{ }^{1} J_{C-F}=269.5 \mathrm{~Hz}\right), 122.8,119.5,117.2,117.1\left(\mathrm{~d},{ }^{4} J_{C-F}=3.8\right.$ $\mathrm{Hz}), 117.0\left(\mathrm{~d},{ }^{4} J_{C-F}=3.8 \mathrm{~Hz}\right), 112.5\left(\mathrm{~d},{ }^{4} J_{C-F}=3.7 \mathrm{~Hz}\right), 112.4\left(\mathrm{~d},{ }^{4} J_{C-F}=3.7 \mathrm{~Hz}\right), 105.3\left(\mathrm{~d},{ }^{2} J_{C-F}\right.$ 
$=25.2 \mathrm{~Hz}), 105.2\left(\mathrm{~d},{ }^{2} J_{C-F}=25.3 \mathrm{~Hz}\right)$; IR (KBr) $\bar{v} 3011,1793,1578,1056,761$; HRMS (ESI/QTOF) m/z: $[\mathrm{M}+\mathrm{Na}]^{+}$calcd for $\mathrm{C}_{15} \mathrm{H}_{7} \mathrm{ClF}_{2} \mathrm{O}_{2} \mathrm{Na}$ 314.9995; found 315.0002 .

3-Chloro-4-(3-(trifluoromethyl)phenyl)-2H-chromen-2-one (4d): $\mathrm{R}_{\mathrm{f}}=0.45$ (10\% ethyl acetate<smiles>O=c1oc2ccccc2c(-c2cccc(C(F)(F)F)c2)c1Cl</smiles>
in hexane); pale yellow solid; yield 66\% (44 mg); mp 105-110 ${ }^{\circ} \mathrm{C}$; ${ }^{1} \mathrm{H}$ NMR $\left(700 \mathrm{MHz}, \mathrm{CDCl}_{3}\right) \delta 7.82(\mathrm{~d}, J=7.9 \mathrm{~Hz}, 1 \mathrm{H}), 7.73(\mathrm{t}, J=7.9 \mathrm{~Hz}, 1 \mathrm{H}), 7.62(\mathrm{~s}$, 1H), 7.59-7.57 (m, 1H), $7.55(\mathrm{~d}, J=7.6 \mathrm{~Hz}, 1 \mathrm{H}), 7.44(\mathrm{~d}, J=8.3 \mathrm{~Hz}, 1 \mathrm{H}), 7.25-$ $7.23(\mathrm{~m}, 1 \mathrm{H}), 7.02(\mathrm{dd}, J=8.0,1.2 \mathrm{~Hz}, 1 \mathrm{H}) ;{ }^{13} \mathrm{C} \mathrm{NMR}\left(175 \mathrm{MHz}, \mathrm{CDCl}_{3}\right) \delta$ $157.2,152.2,149.6,134.0,132.3,132.1,131.7\left(\mathrm{q},{ }^{2} J_{C F 3}=32.9 \mathrm{~Hz}\right), 129.8,127.0,126.5\left(\mathrm{q},{ }^{4} J_{C F 3}\right.$ $=3.5 \mathrm{~Hz}), 125.5\left(\mathrm{q},{ }^{4} J_{C F 3}=3.9 \mathrm{~Hz}\right), 125.2,123.8\left(\mathrm{q},{ }^{1} J_{C F 3}=272.6 \mathrm{~Hz}\right), 121.3,119.8,117.3 ; \mathrm{IR}$ $(\mathrm{KBr}) \bar{v}$ 2923, 1735, 1316, 1129, 757; HRMS (ESI/Q-TOF) m/z: $[\mathrm{M}+\mathrm{Na}]^{+}$calcd for $\mathrm{C}_{16} \mathrm{H}_{8} \mathrm{ClF}_{3} \mathrm{O}_{2} \mathrm{Na} 347.0057$; found 347.0057.

4-(3-Chloro-2-oxo-2H-chromen-4-yl)benzonitrile (4e): $\mathrm{R}_{\mathrm{f}}=0.45(10 \%$ ethyl acetate in<smiles>N#Cc1ccc(-c2c(Cl)c(=O)oc3ccccc23)cc1</smiles>
hexane); white solid; yield 70\% (48 mg); mp $136-138{ }^{\circ} \mathrm{C}$; ${ }^{1} \mathrm{H}$ NMR (400 MHz, $\left.\mathrm{CDCl}_{3}\right) \delta 7.89(\mathrm{~d}, J=8.4 \mathrm{~Hz}, 2 \mathrm{H}), 7.61-7.57(\mathrm{~m}, 1 \mathrm{H}), 7.49(\mathrm{~d}, J=8.4 \mathrm{~Hz}, 2 \mathrm{H})$, $7.45(\mathrm{dd}, J=8.3,0.7 \mathrm{~Hz}, 1 \mathrm{H}), 7.24-7.22(\mathrm{~m}, 1 \mathrm{H}), 6.97(\mathrm{dd}, J=8.0,1.4 \mathrm{~Hz}$, $1 \mathrm{H}) ;{ }^{13} \mathrm{C}$ NMR $\left(175 \mathrm{MHz}, \mathrm{CDCl}_{3}\right) \delta 157.0,152.2,149.2,137.8,132.9,132.5$, 129.6, 126.8, 125.2, 121.2, 119.4, 118.1, 117.4, 113.8; IR (KBr) v̄ 3080, 1732, 1568, 1317, 765; HRMS (ESI/Q-TOF) m/z: [M + Na] $]^{+}$calcd for $\mathrm{C}_{16} \mathrm{H}_{8} \mathrm{ClNO}_{2} \mathrm{Na}$ 304.0136; found 304.0143.

2-(3-Chloro-2-oxo-2H-chromen-4-yl)benzonitrile $\mathbf{( 4 f ) :} \mathrm{R}_{\mathrm{f}}=0.55(10 \%$ ethyl acetate in<smiles>N#Cc1ccccc1-c1c(Cl)c(=O)oc2ccccc12</smiles>
hexane); white solid; yield 51\% (35 mg); mp 120-124 ${ }^{\circ} \mathrm{C}$; ${ }^{1} \mathrm{H}$ NMR $(700 \mathrm{MHz}$, 
$\left.\mathrm{CDCl}_{3}\right) \delta 7.92(\mathrm{~d}, J=7.8 \mathrm{~Hz}, 1 \mathrm{H}), 7.83(\mathrm{t}, J=7.7 \mathrm{~Hz}, 1 \mathrm{H}), 7.69(\mathrm{t}, J=7.8 \mathrm{~Hz}, 1 \mathrm{H}), 7.59(\mathrm{t}, J=$ $7.8 \mathrm{~Hz}, 1 \mathrm{H}), 7.46(\mathrm{~d}, J=8.3 \mathrm{~Hz}, 1 \mathrm{H}), 7.44(\mathrm{~d}, J=7.8 \mathrm{~Hz}, 1 \mathrm{H}), 7.24(\mathrm{t}, J=7.6 \mathrm{~Hz}, 1 \mathrm{H}), 6.88(\mathrm{~d}, J$ $=8.0 \mathrm{~Hz}, 1 \mathrm{H}) ;{ }^{13} \mathrm{C} \mathrm{NMR}\left(175 \mathrm{MHz}, \mathrm{CDCl}_{3}\right) \delta 156.8,152.2,147.6,137.0,133.7,133.5,132.6$, 130.2, 129.5, 126.5, 125.3, 122.5, 119.3, 117.4, 116.4, 112.5; IR (KBr) v 3088, 1736, 1562, 1321, 726; HRMS (ESI/Q-TOF) m/z: $[\mathrm{M}+\mathrm{H}]^{+}$calcd for $\mathrm{C}_{16} \mathrm{H}_{9} \mathrm{ClNO}_{2}$ 282.0316; found 282.0328 .

3-Chloro-4-(4-nitrophenyl)-2H-chromen-2-one (4g): $\mathrm{R}_{\mathrm{f}}=0.35$ (10\% ethyl acetate in hexane);

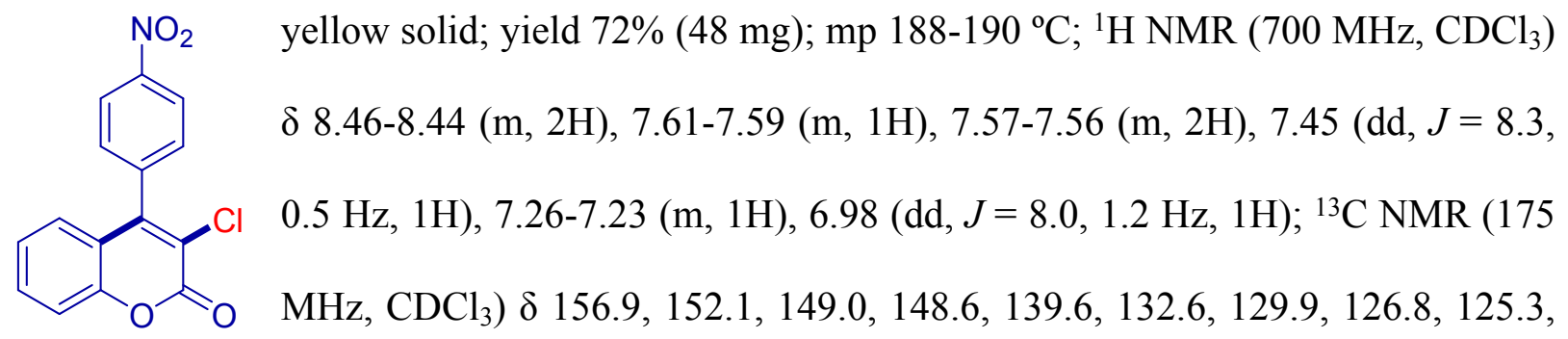

124.4, 121.2, 119.3, 117.4; IR (KBr) v 3012, 3045, 1785, 1644, 768; HRMS (ESI/Q-TOF) m/z: $[\mathrm{M}+\mathrm{H}]^{+}$calcd for $\mathrm{C}_{15} \mathrm{H}_{9} \mathrm{ClNO}_{4} 302.0215$; found 302.0205 .

3-Chloro-4-(naphthalen-1-yl)-2H-chromen-2-one (4h): $\mathrm{R}_{\mathrm{f}}=0.45(10 \%$ ethyl acetate in $\left.\mathrm{CDCl}_{3}\right) \delta 8.04(\mathrm{~d}, J=8.3 \mathrm{~Hz}, 1 \mathrm{H}), 7.98(\mathrm{~d}, J=8.3 \mathrm{~Hz}, 1 \mathrm{H}), 7.65(\mathrm{t}, J=7.6$
$\mathrm{Hz}, 1 \mathrm{H}), 7.56-7.52(\mathrm{~m}, 2 \mathrm{H}), 7.47(\mathrm{~d}, J=8.4 \mathrm{~Hz}, 1 \mathrm{H}), 7.45(\mathrm{~d}, J=3.8 \mathrm{~Hz}, 2 \mathrm{H})$, $7.40(\mathrm{~d}, J=6.9 \mathrm{~Hz}, 1 \mathrm{H}), 7.10(\mathrm{t}, J=7.6 \mathrm{~Hz}, 1 \mathrm{H}), 6.84(\mathrm{~d}, J=8.0 \mathrm{~Hz}, 1 \mathrm{H}) ;{ }^{13} \mathrm{C}$ NMR $(175 \mathrm{MHz}$, $\left.\mathrm{CDCl}_{3}\right) \delta 157.6,152.1,150.7,133.7,132.1,130.9,130.1,130.0,128.9,127.7,127.4,126.8$, 126.5, 125.5, 125.0, 124.8, 122.2, 120.6, 117.0; IR (KBr) $\bar{v} 3055,1736,1056,755$; HRMS (ESI/Q-TOF) m/z: [M + Na $]^{+}$calcd for $\mathrm{C}_{19} \mathrm{H}_{11} \mathrm{ClO}_{2} \mathrm{Na}$ 329.0340; found 329.0361. 
4-([1,1'-Biphenyl]-4-yl)-3-chloro-2H-chromen-2-one (4i): $\mathrm{R}_{\mathrm{f}}=0.55(10 \%$ ethyl acetate in

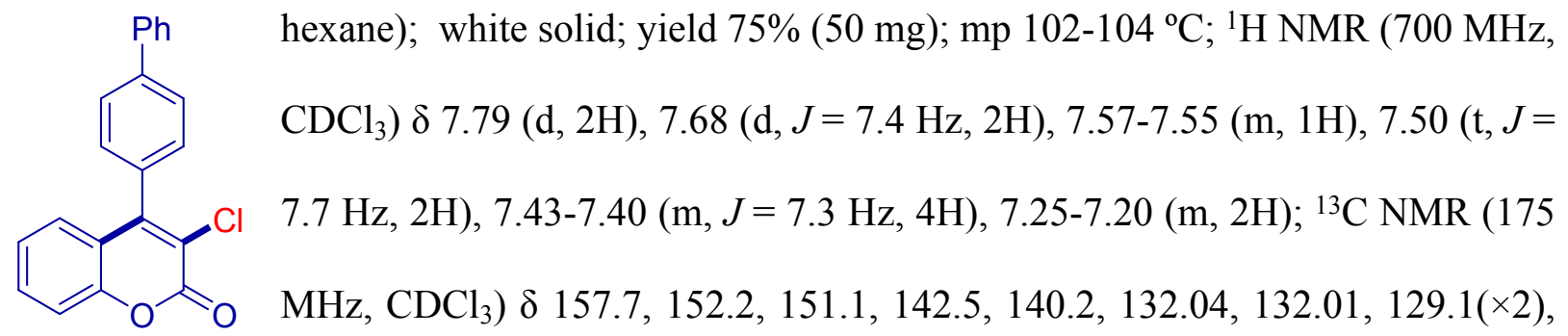

128.1, 127.7, 127.6, 127.3, 124.9, 120.8, 120.3, 117.1; IR (KBr) v̄ 3028, 2912, 1774, 1609, 744;

HRMS (ESI/Q-TOF) m/z: [M + Na $]^{+}$calcd for $\mathrm{C}_{21} \mathrm{H}_{13} \mathrm{ClO}_{2} \mathrm{Na} 355.0496$; found 355.0467.

4-([1,1'-Biphenyl]-2-yl)-3-chloro-2H-chromen-2-one $(\mathbf{4 j}): \mathrm{R}_{\mathrm{f}}=0.65(10 \%$ ethyl acetate in<smiles>O=c1oc2ccccc2c(-c2ccccc2-c2ccccc2)c1Cl</smiles>
hexane); semi solid; yield 54\% (36 mg); $\left.{ }^{1} \mathrm{H} \mathrm{NMR} \mathrm{(700} \mathrm{MHz,} \mathrm{CDCl}_{3}\right) \delta 7.69(\mathrm{t}$, $J=7.6 \mathrm{~Hz}, 1 \mathrm{H}), 7.63-7.62(\mathrm{~m}, 2 \mathrm{H}), 7.55(\mathrm{t}, J=7.8 \mathrm{~Hz}, 1 \mathrm{H}), 7.39(\mathrm{~d}, J=8.3$ $\mathrm{Hz}, 1 \mathrm{H}), 7.36-7.34(\mathrm{~m}, 2 \mathrm{H}), 7.24-7.22(\mathrm{~m}, 5 \mathrm{H}), 7.13(\mathrm{~d}, J=7.9 \mathrm{~Hz}, 1 \mathrm{H}) ;{ }^{13} \mathrm{C}$ NMR $\left(175 \mathrm{MHz}, \mathrm{CDCl}_{3}\right) \delta 157.5,151.8,151.7,141.4,139.9,131.8,131.8,130.8,130.0,129.0$, $128.4,128.3,127.9,127.8,127.5,124.79,121.6,120.3,116.9$; IR (KBr) v 3094, 2933, 1767 , 1599, 751; HRMS (ESI/Q-TOF) m/z: $[\mathrm{M}+\mathrm{Na}]^{+}$calcd for $\mathrm{C}_{21} \mathrm{H}_{13} \mathrm{ClO}_{2} \mathrm{Na}$ 355.0496; found 355.0481 .

3-Chloro-4-(4-methoxyphenyl)-2H-chromen-2-one (4k): $\mathrm{R}_{\mathrm{f}}=0.55(10 \%$ ethyl acetate in<smiles>COc1ccc(-c2c(Cl)c(=O)oc3ccccc23)cc1</smiles>
hexane); white solid; yield 62\% (42 mg); mp 162-168 ${ }^{\circ} \mathrm{C} ;{ }^{1} \mathrm{H}$ NMR (700 MHz, $\left.\mathrm{CDCl}_{3}\right) \delta 7.54(\mathrm{t}, J=7.6 \mathrm{~Hz}, 1 \mathrm{H}), 7.41(\mathrm{~d}, J=8.3 \mathrm{~Hz}, 1 \mathrm{H}), 7.29(\mathrm{~d}, J=8.3 \mathrm{~Hz}$ 2H), 7.20-7.18 (m, 2H), $7.08(\mathrm{~d}, J=8.3 \mathrm{~Hz}, 2 \mathrm{H}), 3.91(\mathrm{~s}, 3 \mathrm{H}) ;{ }^{13} \mathrm{C}$ NMR $(175$ $\left.\mathrm{MHz}, \mathrm{CDCl}_{3}\right) \delta 160.5,157.8,152.2,151.1,131.9,130.2,127.7,125.2,124.8$ 
120.7, 120.5, 117.0, 114.4, 55.5; IR (KBr) v̄ 3020, 2839, 1745, 1587, 1042, 772; HRMS (ESI/QTOF) m/z: [M + Na] calcd for $\mathrm{C}_{16} \mathrm{H}_{11} \mathrm{ClO}_{3} \mathrm{Na}$ 309.0289; found 309.0291.

3-Chloro-4-(pyridin-3-yl)-2H-chromen-2-one (4l): $\mathrm{R}_{\mathrm{f}}=0.35$ (20\% ethyl acetate in hexane);<smiles>O=c1oc2ccccc2c(-c2cccnc2)c1Cl</smiles>
semi solid; yield 87\% (60 mg); ${ }^{1} \mathrm{H}$ NMR $\left(700 \mathrm{MHz}, \mathrm{CDCl}_{3}\right) \delta 8.85$ (d, $J=4.8$ $\mathrm{Hz}, 1 \mathrm{H}), 8.67(\mathrm{~s}, 1 \mathrm{H}), 7.81(\mathrm{~d}, J=7.8 \mathrm{~Hz}, 1 \mathrm{H}), 7.63-7.60(\mathrm{~m}, 2 \mathrm{H}), 7.47$ (d, $J=$ $8.3 \mathrm{~Hz}, 1 \mathrm{H}), 7.28-7.26(\mathrm{~m}, 1 \mathrm{H}), 7.07(\mathrm{~d}, J=8.0 \mathrm{~Hz}, 1 \mathrm{H}) ;{ }^{13} \mathrm{C} \mathrm{NMR}(175 \mathrm{MHz}$, $\left.\mathrm{CDCl}_{3}\right) \delta 157.0,152.2,150.1,148.4,147.4,137.3,132.5,129.8,126.8,125.3,124.1,122.0$, 119.6, 117.4; IR (KBr) $\bar{v} 3071,1730,1585,1042,788$; HRMS (ESI/Q-TOF) m/z: $[\mathrm{M}+\mathrm{H}]^{+}$ calcd for $\mathrm{C}_{14} \mathrm{H}_{9} \mathrm{ClNO}_{2} 258.0316$; found 258.0303.

3-chloro-4-(thiophen-3-yl)-2H-chromen-2-one (4m): $\mathrm{R}_{\mathrm{f}}=0.6(10 \%$ ethyl acetate in hexane);<smiles>O=c1oc2ccccc2c(-c2ccsc2)c1Cl</smiles>
semi solid; yield 32\% (22 mg); ${ }^{1} \mathrm{H}$ NMR (700 MHz, $\left.\mathrm{CDCl}_{3}\right) \delta$ 7.56-7.54 (m, 2H), $7.50(\mathrm{~d}, J=2.2 \mathrm{~Hz}, 1 \mathrm{H}), 7.41(\mathrm{~d}, J=8.3 \mathrm{~Hz}, 1 \mathrm{H}), 7.31(\mathrm{~d}, J=7.9 \mathrm{~Hz}$, 1H), 7.27-7.23 (m, 1H), 7.19 (d, $\left.J=4.9 \mathrm{~Hz}, 1 \mathrm{H}) ;{ }^{13} \mathrm{C} \mathrm{NMR} \mathrm{(175} \mathrm{MHz,} \mathrm{CDCl}_{3}\right)$ $\delta 157.6,152.0,146.9,132.6,132.0,128.2,127.4,126.8,126.7,124.9,121.0,120.1,117.1$; IR $(\mathrm{KBr}) \bar{v}$ 3090, 1741, 1622, 1044, 756; HRMS (ESI/Q-TOF) m/z: $[\mathrm{M}+\mathrm{Na}]^{+}$calcd for $\mathrm{C}_{13} \mathrm{H}_{7} \mathrm{ClO}_{2} \mathrm{SNa} 284.9747$; found 284.9764 .

Phenyl 3-phenylpropiolate (1a): ${ }^{1} \mathrm{R}_{\mathrm{f}}=0.8$ (5\% ethyl acetate in hexane); white solid; yield 752<smiles>O=C(C#Cc1ccccc1)Oc1ccccc1</smiles>
mg (64\%); NMR (400 MHz, $\left.\mathrm{CDCl}_{3}\right) \delta$ 7.61-7.59 (m, 2H), 7.48-7.43 (m, 1H), 7.42-7.34 (m, 4H), 7.27-7.23 (m, 1H), 7.18-7.16 (m, 2H). 
4-Bromophenyl 3-phenylpropiolate (1b): ${ }^{1} \mathrm{R}_{\mathrm{f}}=0.7$ (5\% ethyl acetate in hexane); white solid;

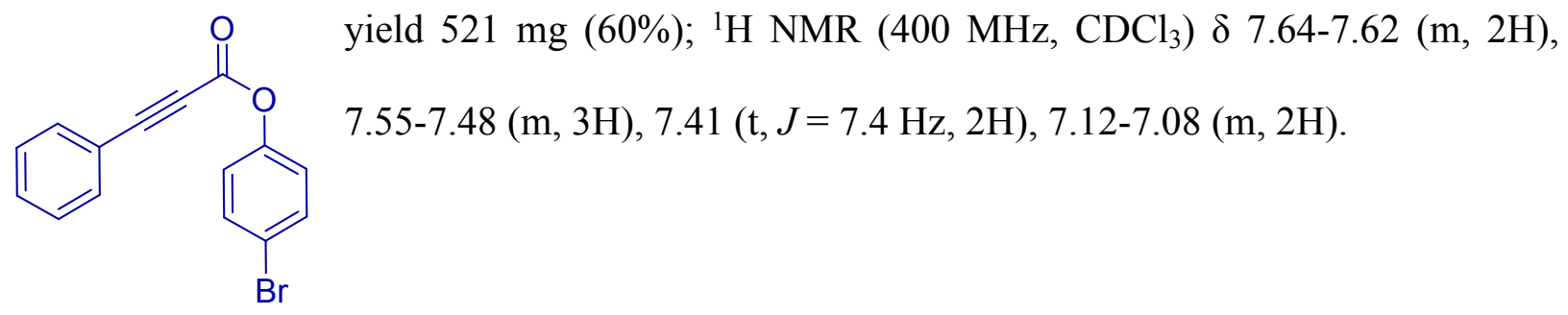

4-Chlorophenyl 3-phenylpropiolate (1c): ${ }^{1} \mathrm{R}_{\mathrm{f}}=0.7$ (5\% ethyl acetate in hexane); white solid;

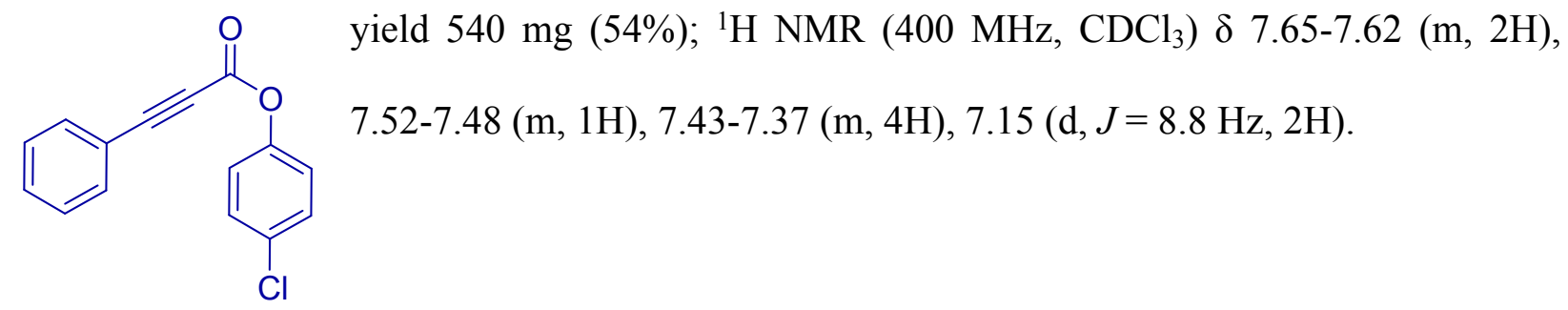

4-Fluorophenyl 3-phenylpropiolate (1d): ${ }^{9} \mathrm{R}_{\mathrm{f}}=0.6$ (5\% ethyl acetate in hexane); white solid;

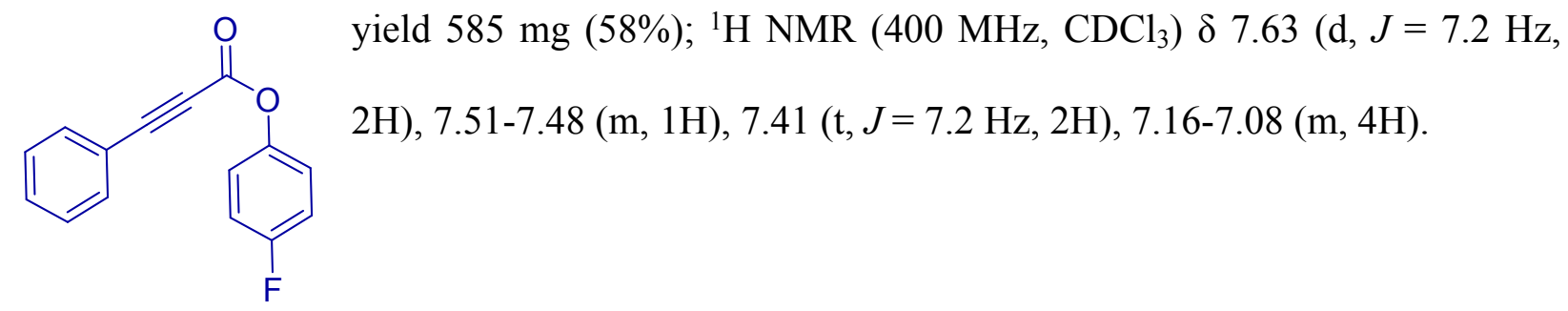

4-(Trifluoromethyl)phenyl 3-phenylpropiolate (1e): ${ }^{10} \mathrm{R}_{\mathrm{f}}=0.45$ (5\% ethyl acetate in hexane);

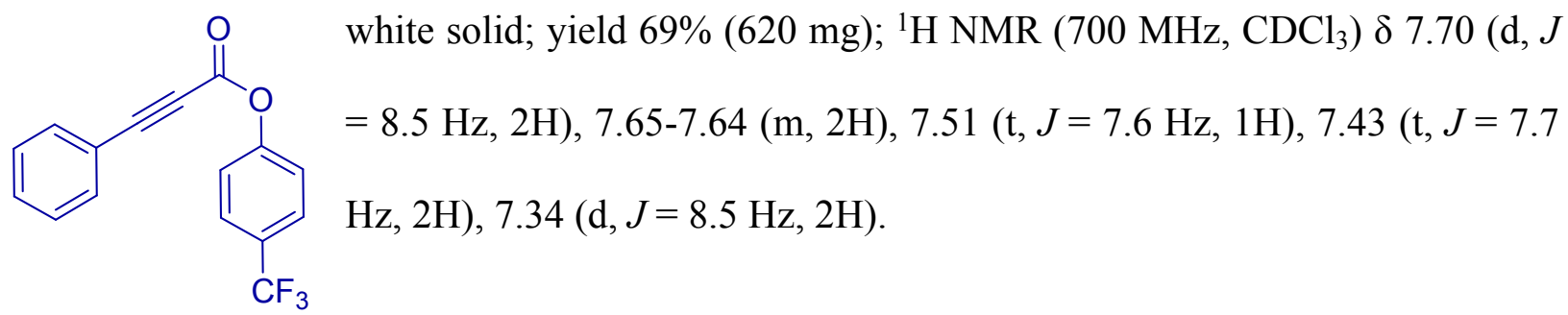

3-(Trifluoromethyl)phenyl 3-phenylpropiolate (1f): ${ }^{10} \mathrm{R}_{\mathrm{f}}=0.45$ (5\% ethyl acetate in hexane);<smiles>O=C(C#Cc1ccccc1)Oc1cccc(C(F)(F)F)c1</smiles>
white solid; yield 65\% (580 mg); ${ }^{1} \mathrm{H} \mathrm{NMR}\left(400 \mathrm{MHz}, \mathrm{CDCl}_{3}\right) \delta 7.66-$ 
$7.64(\mathrm{~m}, 2 \mathrm{H}), 7.55(\mathrm{~d}, J=5.2 \mathrm{~Hz}, 2 \mathrm{H}), 7.53-7.49(\mathrm{~m}, 2 \mathrm{H}), 7.44-7.40(\mathrm{~m}, 3 \mathrm{H})$.

4-(Trifluoromethoxy)phenyl 3-phenylpropiolate (1g): ${ }^{10} \mathrm{R}_{\mathrm{f}}=0.45(20 \%$ ethyl acetate in

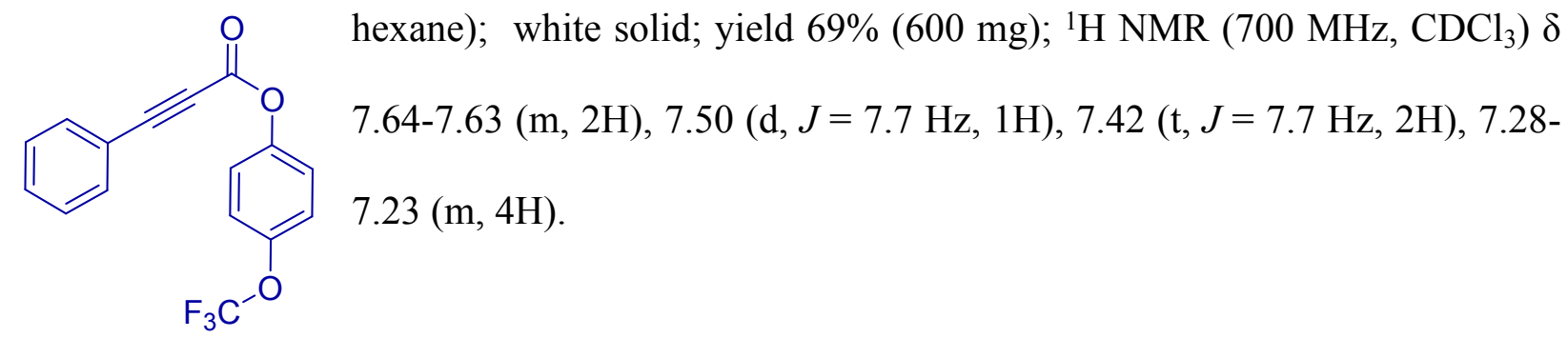

4-Cyanophenyl 3-phenylpropiolate (1h): ${ }^{1} \mathrm{R}_{\mathrm{f}}=0.4$ (5\% ethyl acetate in hexane); white solid;

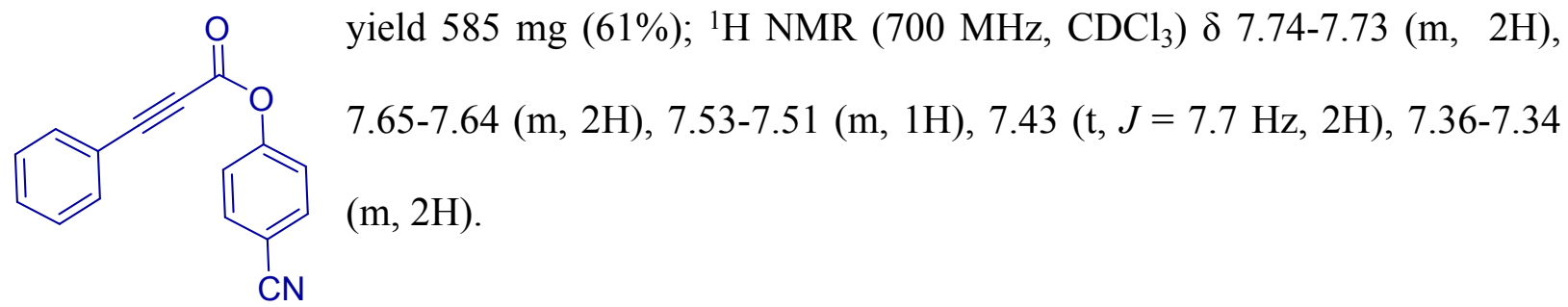

3-Cyanophenyl 3-phenylpropiolate (1i): $\mathrm{R}_{\mathrm{f}}=0.45$ (5\% ethyl acetate in hexane); white semi<smiles>N#Cc1cccc(OC(=O)C#Cc2ccccc2)c1</smiles>
solid; yield 77\% (800 mg); ${ }^{1} \mathrm{H}$ NMR $\left(700 \mathrm{MHz}, \mathrm{CDCl}_{3}\right) \delta$ 7.66-7.65 (m, 2H), 7.59-7.58 (m, 1H), 7.55-7.51 (m, 3H), 7.48-7.46 (m, 1H), $7.43(\mathrm{t}, J=7.7 \mathrm{~Hz}, 2 \mathrm{H}) ;{ }^{13} \mathrm{C}$ NMR $\left(175 \mathrm{MHz}, \mathrm{CDCl}_{3}\right) \delta$ 151.6, 150.3, 133.5, 131.5, 130.7, 130.2, 128.9, 126.6, 125.4, 119.1, 117.8, 113.8, 90.0, 79.8; IR (KBr) $\bar{v} 3074$, 2925, 2219, 1730; HRMS (ESI/Q-TOF) m/z: $[\mathrm{M}+\mathrm{H}]^{+}$calcd for $\mathrm{C}_{16} \mathrm{H}_{10} \mathrm{NO}_{2}$ 248.0706; found 248.0687. 
4-Nitrophenyl 3-phenylpropiolate $(\mathbf{1 j}):^{11} \mathrm{R}_{\mathrm{f}}=0.4$ (5\% ethyl acetate in hexane); yellowish<smiles>O=C(C#Cc1ccccc1)Oc1ccc([N+](=O)[O-])cc1</smiles>
white solid; yield $598 \mathrm{mg}(63 \%) ;{ }^{1} \mathrm{H}$ NMR (400 MHz, $\left.\mathrm{CDCl}_{3}\right) \delta 8.31(\mathrm{~d}, J$ $=9.0 \mathrm{~Hz}, 2 \mathrm{H}), 7.67-7.65(\mathrm{~m}, 2 \mathrm{H}), 7.55-7.51(\mathrm{~m}, 1 \mathrm{H}), 7.45-7.38(\mathrm{~m}, 4 \mathrm{H})$.

3-Nitrophenyl 3-phenylpropiolate (1k): $\mathrm{R}_{\mathrm{f}}=0.5(10 \%$ ethyl acetate in hexane); semi solid;<smiles>O=C(C#Cc1ccccc1)Oc1cccc([N+](=O)[O-])c1</smiles>
yield 52\% (501 mg); ${ }^{1} \mathrm{H}$ NMR (700 $\left.\mathrm{MHz}, \mathrm{CDCl}_{3}\right) \delta 8.17-8.16(\mathrm{~m}$, $1 \mathrm{H}), 8.11(\mathrm{t}, J=2.1 \mathrm{~Hz}, 1 \mathrm{H}), 7.67-7.65(\mathrm{~m}, 2 \mathrm{H}), 7.61(\mathrm{t}, J=8.1 \mathrm{~Hz}$, 1H), 7.58-7.56 (m, 1H), 7.53-7.51 (m, 1H), $7.43(\mathrm{t}, J=7.7 \mathrm{~Hz}, 2 \mathrm{H})$; ${ }^{13} \mathrm{C}$ NMR $\left(175 \mathrm{MHz}, \mathrm{CDCl}_{3}\right) \delta 151.6,150.4,149.0,133.5,131.6,130.4,128.9,128.0,121.4$, 118.9, 117.4, 90.2, 79.7; IR (KBr) v̄ 3001, 2215, 1729, 1530, 1141; HRMS (ESI/Q-TOF) m/z: $[\mathrm{M}+\mathrm{Na}]^{+}$calcd for $\mathrm{C}_{15} \mathrm{H}_{9} \mathrm{NO}_{4} \mathrm{Na} 290.0424$; found 290.0422 .

Naphthalen-2-yl 3-phenylpropiolate (1I): ${ }^{1} \mathrm{R}_{\mathrm{f}}=0.7$ (5\% ethyl acetate in hexane); yellowish

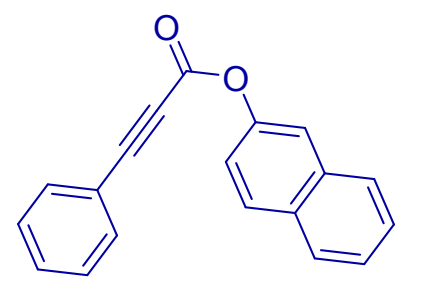
white solid; yield $570 \mathrm{mg}(60 \%) ;{ }^{1} \mathrm{H} \mathrm{NMR}\left(400 \mathrm{MHz}, \mathrm{CDCl}_{3}\right) \delta 7.92-$ $7.84(\mathrm{~m}, 3 \mathrm{H}), 7.70-7.65(\mathrm{~m}, 3 \mathrm{H}), 7.54-7.50(\mathrm{~m}, 3 \mathrm{H}), 7.44-7.40(\mathrm{~m}$, 2H), $7.35(\mathrm{dd}, J=8.8,2.2 \mathrm{~Hz}, 1 \mathrm{H})$.

[1,1'-Biphenyl]-4-yl 3-phenylpropiolate $(1 \mathrm{~m}):^{1} \mathrm{R}_{\mathrm{f}}=0.6(5 \%$ ethyl acetate in hexane); white

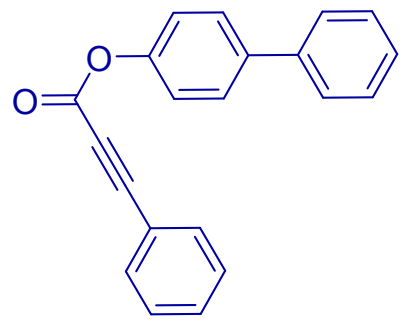
solid; yield 74\% (650 mg); ${ }^{1} \mathrm{H}$ NMR (400 MHz, $\left.\mathrm{CDCl}_{3}\right) \delta 7.53(\mathrm{t}, J=$ $7.6 \mathrm{~Hz}, 4 \mathrm{H}), 7.47(\mathrm{~d}, J=7.6 \mathrm{~Hz}, 2 \mathrm{H}), 7.41-7.31(\mathrm{~m}, 3 \mathrm{H}), 7.29-7.24$ (m, 1H), 7.18-7.15 (m, 4H). 
4-Tritylphenyl 3-phenylpropiolate (1n): $\mathrm{R}_{\mathrm{f}}=0.55$ (10\% ethyl acetate in hexane); white solid;

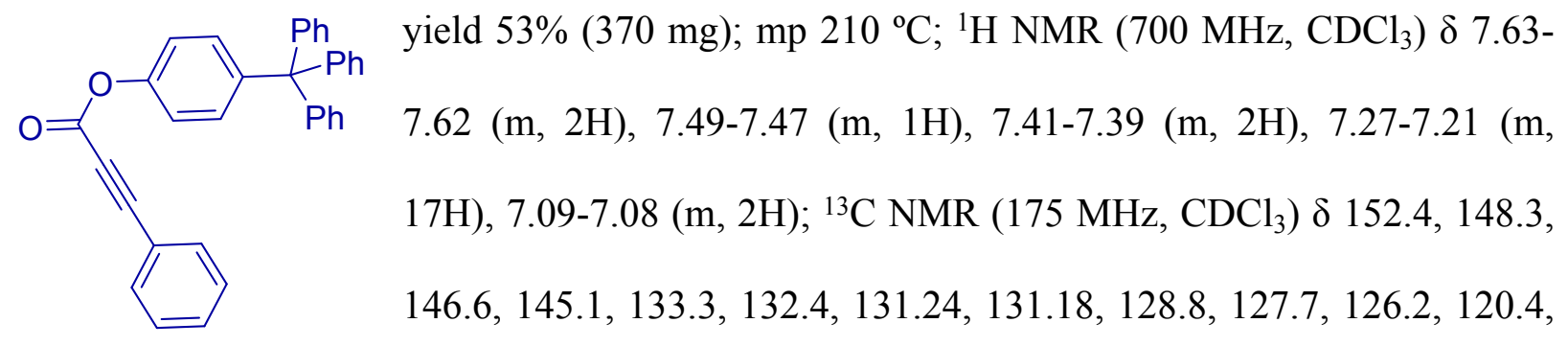

119.4, 88.9, 80.4, 64.8; IR (KBr) v 3063, 2231, 1726, 1492, 1170; HRMS (ESI/Q-TOF) m/z: [M

$+\mathrm{Na}^{+}$calcd for $\mathrm{C}_{34} \mathrm{H}_{24} \mathrm{O}_{2} \mathrm{Na} 487.1669$; found 487.1660 .

p-Tolyl 3-phenylpropiolate (10): ${ }^{1} \mathrm{R}_{\mathrm{f}}=0.6$ (5\% ethyl acetate in hexane); white solid; yield 625

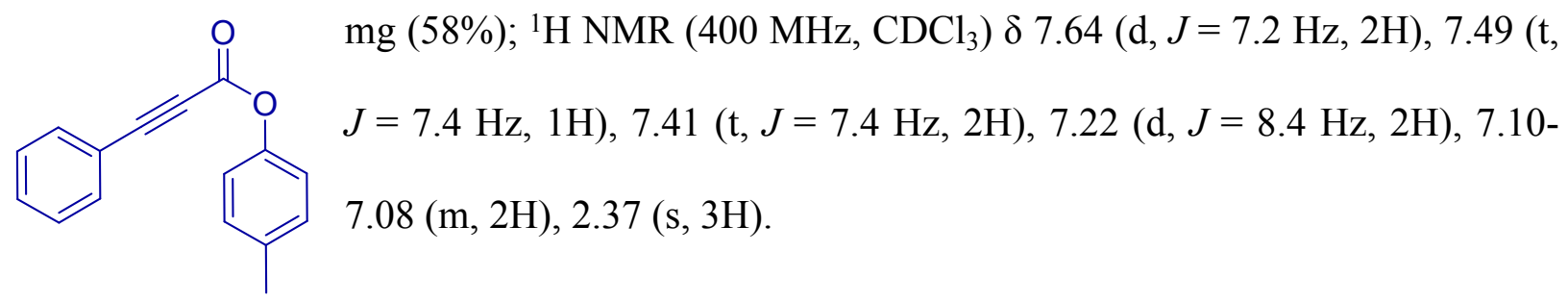

4-(tert-Butyl)phenyl 3-phenylpropiolate (1p): ${ }^{10} \mathrm{R}_{\mathrm{f}}=0.8$ (5\% ethyl acetate in hexane); white

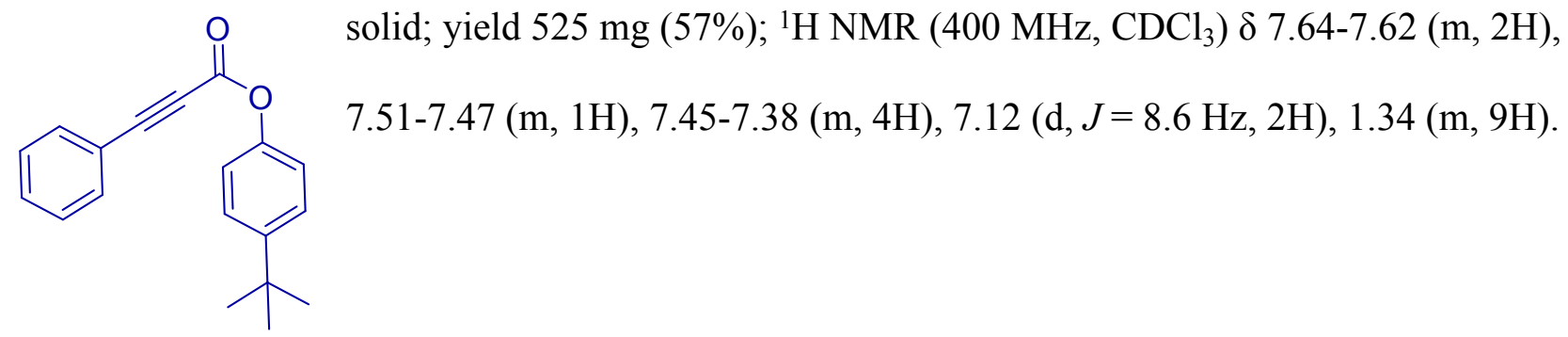

4-Ethylphenyl 3-phenylpropiolate (1q): ${ }^{10} \mathrm{R}_{\mathrm{f}}=0.7$ (5\% ethyl acetate in hexane); yellowish<smiles>CCc1ccc(OC(=O)C#Cc2ccccc2)cc1</smiles>
white solid; yield $630 \mathrm{mg}$ (62\%); mp: $51-53{ }^{\circ} \mathrm{C}$; ${ }^{1} \mathrm{H}$ NMR $(400 \mathrm{MHz}$, $\left.\mathrm{CDCl}_{3}\right) \delta 7.49(\mathrm{~d}, J=7.4 \mathrm{~Hz}, 2 \mathrm{H}), 7.34-7.32(\mathrm{~m}, 1 \mathrm{H}), 7.28-7.24(\mathrm{~m}, 2 \mathrm{H})$, $7.10(\mathrm{~d}, J=7.4 \mathrm{~Hz}, 2 \mathrm{H}), 6.97(\mathrm{~d}, J=7.4 \mathrm{~Hz}, 2 \mathrm{H}), 2.55-2.50(\mathrm{~m}, 2 \mathrm{H}), 1.13-$ $1.09(\mathrm{~m}, 3 \mathrm{H})$. 
3-Methoxyphenyl 3-phenylpropiolate (1s). ${ }^{12} \mathrm{R}_{\mathrm{f}}=0.6$ (5\% ethyl acetate in hexane); colorless<smiles>COc1cccc(OC(=O)C#Cc2ccccc2)c1</smiles>
liquid; yield 58\% (693 mg); ${ }^{1} \mathrm{H}$ NMR (400 MHz, $\left.\mathrm{CDCl}_{3}\right) \delta 7.64-$ $7.62(\mathrm{~m}, 2 \mathrm{H}), 7.53-7.47(\mathrm{~m}, 1 \mathrm{H}), 7.43-7.39(\mathrm{~m}, 2 \mathrm{H}), 7.31(\mathrm{t}, J=8.4$ $\mathrm{Hz}, 1 \mathrm{H}), 6.85-6.79(\mathrm{~m}, 2 \mathrm{H}), 6.75(\mathrm{t}, J=2.0 \mathrm{~Hz}, 1 \mathrm{H}), 3.82(\mathrm{~s}, 3 \mathrm{H})$.

3-Chlorophenyl 3-phenylpropiolate (1t). ${ }^{12} \mathrm{R}_{\mathrm{f}}=0.5$ (5\% ethyl acetate in hexane); yellow liquid;<smiles>O=C(C#Cc1ccccc1)Oc1cccc(Cl)c1</smiles>
yield 63\% (510 mg); ${ }^{1} \mathrm{H}$ NMR (400 MHz, $\left.\mathrm{CDCl}_{3}\right) \delta 7.65-7.63(\mathrm{~m}, 2 \mathrm{H})$, 7.52-7.49 (m, 1H), $7.42(\mathrm{t}, J=7.6 \mathrm{~Hz}, 2 \mathrm{H}), 7.35(\mathrm{t}, J=8.0 \mathrm{~Hz}, 1 \mathrm{H})$, 7.28-7.26 (m, 1H), $7.24(\mathrm{t}, J=2.4 \mathrm{~Hz}, 1 \mathrm{H}), 7.13-7.10(\mathrm{~m}, 1 \mathrm{H})$.

3-Chlorophenyl 3-phenylpropiolate (3a). ${ }^{13} \mathrm{R}_{\mathrm{f}}=0.8$ (5\% ethyl acetate in hexane); yellowish

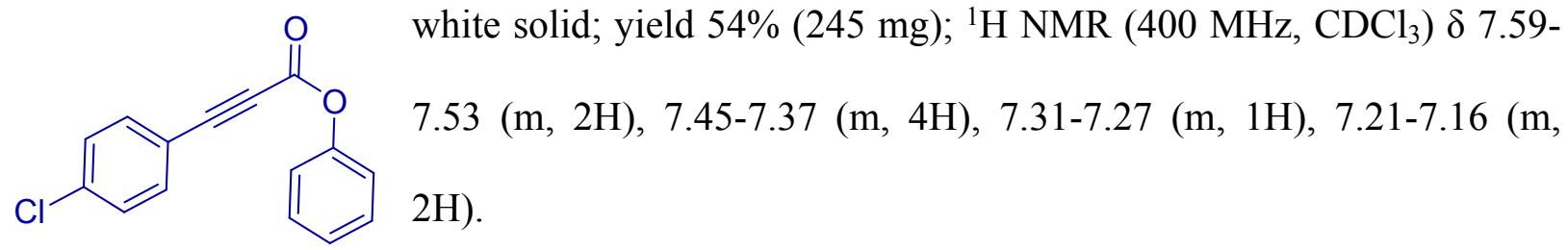

Phenyl 3-(4-fluorophenyl)propiolate (3b): ${ }^{1} \mathrm{R}_{\mathrm{f}}=0.5$ (5\% ethyl acetate in hexane); white solid;

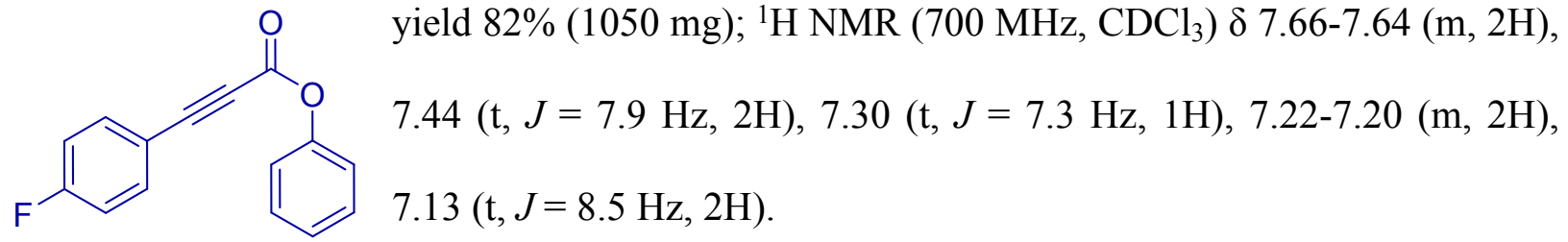

Phenyl 3-(2,4-difluorophenyl)propiolate (3c): $\mathrm{R}_{\mathrm{f}}=0.45$ (5\% ethyl acetate in hexane); white<smiles>O=C(C#Cc1ccc(F)cc1F)Oc1ccccc1</smiles>
solid; yield 58\% (800 mg); mp 78-83 ${ }^{\circ} \mathrm{C} ;{ }^{1} \mathrm{H}$ NMR (700 MHz, $\left.\mathrm{CDCl}_{3}\right) \delta$ 
7.62-7.59 (m, 1H), 7.42 (t, $J=7.8 \mathrm{~Hz}, 2 \mathrm{H}), 7.29-7.27(\mathrm{t}, J=7.3 \mathrm{~Hz}, 1 \mathrm{H}), 7.20(\mathrm{~d}, J=7.7 \mathrm{~Hz}$, 2H), 6.96-7.90 (m, 2H); ${ }^{13} \mathrm{C}$ NMR $\left(175 \mathrm{MHz}, \mathrm{CDCl}_{3}\right) \delta 165.5\left(\mathrm{~d},{ }^{3} J_{C F}=11.5 \mathrm{~Hz}\right), 165.4\left(\mathrm{~d},{ }^{3} J_{C F}\right.$ $=12.5 \mathrm{~Hz}), 164.0\left(\mathrm{~d},{ }^{3} J_{C-F}=11.4 \mathrm{~Hz}\right), 163.9\left(\mathrm{~d},{ }^{3} J_{C-F}=12.5 \mathrm{~Hz}\right), 152.0,150.2,136.1\left(\mathrm{~d},{ }^{3} J_{C-F}=\right.$ $10.3 \mathrm{~Hz}), 129.8,126.6,121.5,112.5\left(\mathrm{~d},{ }^{4} J_{C-F}=3.7 \mathrm{~Hz}\right), 112.4\left(\mathrm{~d},{ }^{4} J_{C-F}=3.7 \mathrm{~Hz}\right), 105.1\left(\mathrm{~d},{ }^{2} J_{C-F}\right.$ $=24.2 \mathrm{~Hz}), 104.9\left(\mathrm{~d},{ }^{2} J_{C-F}=24.3 \mathrm{~Hz}\right), 104.8\left(\mathrm{~d},{ }^{4} J_{C-F}=4.3 \mathrm{~Hz}\right), 104.7\left(\mathrm{~d},{ }^{4} J_{C-F}=4.0 \mathrm{~Hz}\right), 84.8$, 81.1; IR (KBr) $\bar{v} 3020,1728,1590,1222$; HRMS (ESI/Q-TOF) m/z: $[\mathrm{M}+\mathrm{Na}]^{+}$calcd for $\mathrm{C}_{15} \mathrm{H}_{8} \mathrm{~F}_{2} \mathrm{O}_{2} \mathrm{Na} 281.0385$; found 281.0407.

Phenyl 3-(3-(trifluoromethyl)phenyl)propiolate (3d): $\mathrm{R}_{\mathrm{f}}=0.5$ (5\% ethyl acetate in hexane);

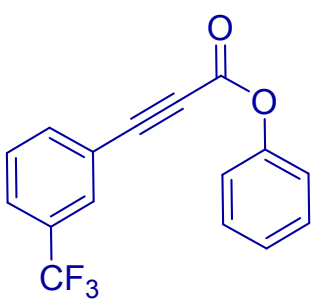
semi solid; yield 75\% (1150 mg); ${ }^{1} \mathrm{H}$ NMR (700 MHz, $\left.\mathrm{CDCl}_{3}\right) \delta 7.90(\mathrm{~s}$, 1H), $7.81(\mathrm{~d}, J=7.7 \mathrm{~Hz}, 1 \mathrm{H}), 7.74(\mathrm{~d}, J=7.9 \mathrm{~Hz}, 1 \mathrm{H}), 7.56(\mathrm{t}, J=7.7 \mathrm{~Hz}$, $1 \mathrm{H}), 7.44-7.42(\mathrm{~m}, 2 \mathrm{H}), 7.29(\mathrm{t}, J=7.5 \mathrm{~Hz}, 1 \mathrm{H}), 7.20-7.19(\mathrm{~m}, 2 \mathrm{H}) ;{ }^{13} \mathrm{C}$ $\operatorname{NMR}\left(175 \mathrm{MHz}, \mathrm{CDCl}_{3}\right) \delta 147.0,145.2,131.2,126.6\left(\mathrm{q},{ }^{2} J_{C F 3}=33.2 \mathrm{~Hz}\right)$, $125.1\left(\mathrm{q},{ }^{4} J_{C F 3}=3.9 \mathrm{~Hz}\right), 124.8,124.5,122.7\left(\mathrm{q},{ }^{4} J C F_{3}=3.4 \mathrm{~Hz}\right), 120.7,118.5\left(\mathrm{q},{ }^{1} J_{C F 3}=272.6\right.$ Hz) $116.5,115.5,81.4,76.3$; IR (KBr) $\bar{v} 2930,2234,1730,1487,1149,805$; HRMS (ESI/QTOF) $\mathrm{m} / \mathrm{z}:[\mathrm{M}+\mathrm{H}]^{+}$calcd for $\mathrm{C}_{16} \mathrm{H}_{10} \mathrm{~F}_{3} \mathrm{O}_{2} 291.0627$; found 291.0605 .

Phenyl 3-(4-cyanophenyl)propiolate (3e): ${ }^{14} \mathrm{R}_{\mathrm{f}}=0.5$ (10\% ethyl acetate in hexane); white solid; yield $68 \%(900 \mathrm{mg}) ;{ }^{1} \mathrm{H} \mathrm{NMR}\left(400 \mathrm{MHz}, \mathrm{CDCl}_{3}\right) \delta$ 7.74-7.71 (m, 4H),
$7.45-7.41(\mathrm{~m}, 2 \mathrm{H}), 7.31-7.29(\mathrm{~m}, 1 \mathrm{H}), 7.20-7.18(\mathrm{~m}, 1 \mathrm{H}), 6.84-6.82$ 
Phenyl 3-(2-cyanophenyl)propiolate (3f): $\mathrm{R}_{\mathrm{f}}=0.55$ (10\% ethyl acetate in hexane); yellow solid; yield $62 \%(820 \mathrm{mg}) ; \mathrm{mp} 83-86{ }^{\circ} \mathrm{C} ;{ }^{1} \mathrm{H}$ NMR $\left(700 \mathrm{MHz}, \mathrm{CDCl}_{3}\right) \delta 7.76(\mathrm{t}, J=7.8 \mathrm{~Hz}, 2 \mathrm{H})$,<smiles>N#Cc1ccccc1C#CC(=O)Oc1ccccc1</smiles>
$7.66(\mathrm{t}, J=7.7 \mathrm{~Hz}, 1 \mathrm{H}), 7.60(\mathrm{t}, J=7.7 \mathrm{~Hz}, 1 \mathrm{H}), 7.43(\mathrm{t}, J=7.6 \mathrm{~Hz}, 2 \mathrm{H})$, $7.29(\mathrm{t}, J=7.2 \mathrm{~Hz}, 1 \mathrm{H}), 7.21(\mathrm{~d}, J=8.3 \mathrm{~Hz}, 2 \mathrm{H}) ;{ }^{13} \mathrm{C} \mathrm{NMR}(175 \mathrm{MHz}$, $\left.\mathrm{CDCl}_{3}\right) \delta 151.6,150.1,134.2,133.3,132.8,131.1,129.8,126.7,123.3$, 121.5, 116.6, 116.5, 85.1, 83.1; IR (KBr) $\bar{v} 3033,1741,1600,1289$; HRMS (ESI/Q-TOF) m/z: $[\mathrm{M}+\mathrm{Na}]^{+}$calcd for $\mathrm{C}_{16} \mathrm{H}_{9} \mathrm{NO}_{2} \mathrm{Na} 270.0525$; found 270.0532 .

Phenyl 3-(4-nitrophenyl)propiolate (3g): ${ }^{14} \mathrm{R}_{\mathrm{f}}=0.55$ (10\% ethyl acetate in hexane); white solid; yield 63\% (900 mg); ${ }^{1} \mathrm{H} \mathrm{NMR}\left(700 \mathrm{MHz}, \mathrm{CDCl}_{3}\right) \delta 8.29(\mathrm{~d}, J=$
$J=7.4 \mathrm{~Hz}, 1 \mathrm{H}), 7.20(\mathrm{~d}, J=7.9 \mathrm{~Hz}, 2 \mathrm{H})$.

Phenyl 3-(naphthalen-1-yl)propiolate (3h): $\mathrm{R}_{\mathrm{f}}=0.5$ (5\% ethyl acetate in hexane); reddish liquid; yield 65\% (780 mg); ${ }^{1} \mathrm{H} \mathrm{NMR}\left(700 \mathrm{MHz}, \mathrm{CDCl}_{3}\right) \delta 8.29(\mathrm{~d}, J=$ $=.3 \mathrm{~Hz}, 1 \mathrm{H}), 7.99(\mathrm{~d}, J=8.3 \mathrm{~Hz}, 1 \mathrm{H}), 7.90(\mathrm{~d}, J=6.9 \mathrm{~Hz}, 2 \mathrm{H}), 7.64(\mathrm{t}, J$
$=7.7 \mathrm{~Hz}, 2 \mathrm{H}), 7.32(\mathrm{t}, J=7.4 \mathrm{~Hz}, 1 \mathrm{H}), 7.27(\mathrm{~d}, J=8.1 \mathrm{~Hz}, 2 \mathrm{H}) ;{ }^{13} \mathrm{C}$ $\operatorname{NMR}\left(175 \mathrm{MHz}, \mathrm{CDCl}_{3}\right) \delta 152.6,150.5,133.8,133.7,133.1,131.9,129.8,128.7,127.9,127.2$ 126.6, 125.8, 125.3, 121.7, 116.9, 87.6, 84.9; IR (KBr) v 3099, 1744, 1602, 1182, 1014; HRMS (ESI/Q-TOF) m/z: [M + Na $]^{+}$calcd for $\mathrm{C}_{19} \mathrm{H}_{12} \mathrm{O}_{2} \mathrm{Na}$ 295.0730; found 295.0748. 
Phenyl 3-([1,1'-biphenyl]-4-yl)propiolate (3i): $\mathrm{R}_{\mathrm{f}}=0.5$ (5\% ethyl acetate in hexane); white<smiles>O=C(C#Cc1ccc(-c2ccccc2)cc1)Oc1ccccc1</smiles>
solid; yield 81\% (1300 mg); mp 105-108 ${ }^{\circ} \mathrm{C}$; ${ }^{1} \mathrm{H}$ NMR (400 MHz, $\left.\mathrm{CDCl}_{3}\right) \delta$ 7.72-7.69 (m, 2H), 7.65-7.59 (m, 4H), 7.50-7.38 (m, 5H), 7.31-7.27 (m, 1H), 7.22-7.20 (m, 2H); ${ }^{13} \mathrm{C}$ NMR (100 MHz, $\left.\mathrm{CDCl}_{3}\right) \delta$ $152.6,150.3,144.0,139.8,133.9,129.7,129.1,128.4,127.5,127.3,126.6,121.6,118.1,88.9$, 81.0; IR (KBr) $\bar{v} 3069,1721,1501,1114,1089$; HRMS (ESI/Q-TOF) m/z: $[\mathrm{M}+\mathrm{H}]^{+}$calcd for $\mathrm{C}_{21} \mathrm{H}_{15} \mathrm{O}_{2} 299.1067$; found 299.1090.

Phenyl 3-([1,1'-biphenyl]-2-yl)propiolate (3j): $\mathrm{R}_{\mathrm{f}}=0.5$ (5\% ethyl acetate in hexane); reddish

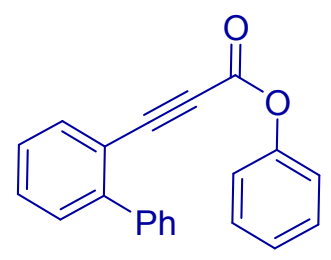
liquid; yield 73\% (1180 mg); ${ }^{1} \mathrm{H}$ NMR $\left(700 \mathrm{MHz}, \mathrm{CDCl}_{3}\right) \delta 7.74(\mathrm{~d}, J=$ $7.7 \mathrm{~Hz}, 1 \mathrm{H}), 7.62(\mathrm{~d}, J=7.5 \mathrm{~Hz}, 2 \mathrm{H}), 7.54(\mathrm{t}, J=7.6 \mathrm{~Hz}, 1 \mathrm{H}), 7.49(\mathrm{t}, J=$ $7.4 \mathrm{~Hz}, 3 \mathrm{H}), 7.42$ (t, $J=7.4 \mathrm{~Hz}, 1 \mathrm{H}), 7.39$ (t, $J=7.8 \mathrm{~Hz}, 3 \mathrm{H}), 7.26-7.24$ (m, 1H), $7.14(\mathrm{~d}, J=7.9 \mathrm{~Hz}, 2 \mathrm{H}) ;{ }^{13} \mathrm{C} \mathrm{NMR}\left(175 \mathrm{MHz}, \mathrm{CDCl}_{3}\right) \delta 152.3,150.3,146.0,139.4$, 134.8, 131.3, 130.0, 129.6, 129.3, 128.4, 128.2, 127.4, 126.4, 121.6, 117.8, 88.8, 82.8; IR (KBr) $\bar{v}$ 3044, 1754, 1574, 1188; HRMS (ESI/Q-TOF) m/z: $[\mathrm{M}+\mathrm{Na}]^{+}$calcd for $\mathrm{C}_{21} \mathrm{H}_{14} \mathrm{O}_{2}$ 321.0886; found 321.0872 .

Phenyl 3-(4-methoxyphenyl)propiolate (3k): ${ }^{13} \mathrm{R}_{\mathrm{f}}=0.6$ (5\% ethyl acetate in hexane); yellowish<smiles>COc1ccc(C#CC(=O)Oc2ccccc2)cc1</smiles>
white solid; yield 47\% (562 mg); ${ }^{1} \mathrm{H}$ NMR (700 MHz, $\left.\mathrm{CDCl}_{3}\right) \delta$ 7.60-7.56 (m, 2H), 7.44-7.38 (m, 2H), $7.29(\mathrm{dt}, J=8.0,1.6 \mathrm{~Hz}$, 1H), 7.20-7.18 (m, 2H), 6.94-6.88 (m, 2H), $3.85(\mathrm{~s}, 3 \mathrm{H})$. 
Phenyl 3-(pyridin-3-yl)propiolate (3l): $\mathrm{R}_{\mathrm{f}}=0.45$ (20\% ethyl acetate in hexane); gray solid;<smiles>O=C(C#Cc1cccnc1)Oc1ccccc1</smiles>
yield $65 \%$ (780 mg); mp $126-128{ }^{\circ} \mathrm{C} ;{ }^{1} \mathrm{H}$ NMR $\left(700 \mathrm{MHz}, \mathrm{CDCl}_{3}\right) \delta 8.85$ (s, 1H), $8.70(\mathrm{~d}, J=4.9 \mathrm{~Hz}, 1 \mathrm{H}), 7.92(\mathrm{~d}, J=7.7 \mathrm{~Hz}, 1 \mathrm{H}), 7.43(\mathrm{t}, J=7.7$ $\mathrm{Hz}, 2 \mathrm{H}), 7.37$ (dd, $J=7.8,5.0 \mathrm{~Hz}, 1 \mathrm{H}), 7.29(\mathrm{t}, J=7.4 \mathrm{~Hz}, 1 \mathrm{H}), 7.20(\mathrm{~d}, J$ $=8.1 \mathrm{~Hz}, 2 \mathrm{H}) ;{ }^{13} \mathrm{C} \mathrm{NMR}\left(175 \mathrm{MHz}, \mathrm{CDCl}_{3}\right) \delta 153.6,152.0,151.2,150.1,140.2,129.8,126.7$, 123.4, 121.5, 116.9, 85.0, 83.2; IR (KBr) v̄ 3034, 1711, 1587, 1124; HRMS (ESI/Q-TOF) m/z: $[\mathrm{M}+\mathrm{H}]^{+}$calcd for $\mathrm{C}_{14} \mathrm{H}_{10} \mathrm{NO}_{2}$ 224.0706; found 224.0712.

Phenyl 3-(thiophen-3-yl)propiolate (3m): $\mathrm{R}_{\mathrm{f}}=0.45$ (5\% ethyl acetate in hexane); reddish<smiles>O=C(C#Cc1ccsc1)Oc1ccccc1</smiles>
liquid; yield 78\% (53 mg); ${ }^{1} \mathrm{H} \mathrm{NMR}\left(400 \mathrm{MHz}, \mathrm{CDCl}_{3}\right) \delta 7.82(\mathrm{dd}, J=3.0$, $1.1 \mathrm{~Hz}, 1 \mathrm{H}), 7.44-7.39(\mathrm{~m}, 2 \mathrm{H}), 7.36-7.34(\mathrm{~m}, 1 \mathrm{H}), 7.30-7.26(\mathrm{~m}, 2 \mathrm{H}), 7.20-$ $7.17(\mathrm{~m}, 2 \mathrm{H}) ;{ }^{13} \mathrm{C}$ NMR $\left(100 \mathrm{MHz}, \mathrm{CDCl}_{3}\right) \delta 152.5,150.2,134.7,130.3$, 129.7, 126.5, 126.4, 121.6, 118.6, 84.3, 80.4; IR (KBr) $\bar{v}$ 3092, 1749, 1620, 1042; HRMS (ESI/Q-TOF) m/z: [M + Na] $]^{+}$calcd for $\mathrm{C}_{13} \mathrm{H}_{8} \mathrm{O}_{2} \mathrm{SNa} 251.0137$; found 251.0133 .

4-Phenyl-2H-chromen-2-one (5): ${ }^{2} \mathrm{R}_{\mathrm{f}}=0.5$ ( $2 \%$ ethyl acetate in hexane); white solid; yield $67 \%$<smiles>O=c1cc(-c2ccccc2)c2ccccc2o1</smiles>
(35 mg); ${ }^{1} \mathrm{H}$ NMR $\left(700 \mathrm{MHz}, \mathrm{CDCl}_{3}\right) \delta$ 7.56-7.52 (m, 4H), $7.50(\mathrm{~d}, J=7.9 \mathrm{~Hz}$, 1H), 7.46-7.45 (m, 2H), 7.42 (d, $J=8.3 \mathrm{~Hz}, 1 \mathrm{H}), 7.23(\mathrm{t}, J=7.6 \mathrm{~Hz}, 1 \mathrm{H}), 6.39$ $(\mathrm{s}, 1 \mathrm{H}) ;{ }^{13} \mathrm{C} \mathrm{NMR}\left(175 \mathrm{MHz}, \mathrm{CDCl}_{3}\right) \delta 160.9,155.8,154.4,135.4,132.1$, $129.8,129.0,128.6,127.2,124.3,119.2,117.5,115.4$.

4-Phenyl-3-(phenylethynyl)-2H-chromen-2-one (6): ${ }^{3} \mathrm{R}_{\mathrm{f}}=0.5$ (5\% ethyl acetate in hexane);<smiles>O=c1oc2ccccc2c(C#Cc2ccccc2)c1-c1ccccc1</smiles>
white solid; yield 52\% (29 mg); ${ }^{1} \mathrm{H}$ NMR (700 MHz, $\left.\mathrm{CDCl}_{3}\right) \delta 7.58$ - 
$7.52(\mathrm{~m}, 4 \mathrm{H}), 7.50(\mathrm{~d}, J=6.8 \mathrm{~Hz}, 2 \mathrm{H}), 7.41(\mathrm{~d}, J=8.3 \mathrm{~Hz}, 1 \mathrm{H}), 7.29-7.25(\mathrm{~m}, 2 \mathrm{H}), 7.26-7.20(\mathrm{~m}$,

5H); ${ }^{13} \mathrm{C} \mathrm{NMR}\left(175 \mathrm{MHz}, \mathrm{CDCl}_{3}\right) \delta 159.6,156.4,153.0,134.5,132.2,131.9,129.5,129.2$,

$128.9,128.6,128.3,127.7,124.6,122.6,119.8,117.1,111.3,98.8,83.9$.

\section{REFERENCES}

(1) Sahoo, H.; Singh, S.; Baidya, M. Radical Cascade Reaction of Aryl Alkynoates at Room Temperature: Synthesis of Fully Substituted A,B-Unsaturated Acids with Chalcogen Functionality. Org. Lett. 2018, 20, 3678-3681.

(2) Xing, L.; Zhang, Y.; Li, B.; Du, Y. Synthesis of 4-Chloroisocoumarins Via Intramolecular Halolactonization of O-Alkynylbenzoates: Phicl2-Mediated C-O/C-Cl Bond Formation. Org. Lett. 2019, 21, 1989-1993.

(3) Sasaki, T.; Moriyama, K.; Togo, H. One-Pot Preparation of 4-Aryl-3-Bromocoumarins from 4-Aryl-2-Propynoic Acids with Diaryliodonium Salts, Tbab, and Na2s2o8. Beilstein J. Org. Chem. 2018, 14, 345-353.

(4) Wang, H.; Li, Y.; Tang, Z.; Wang, S.; Zhang, H.; Cong, H.; Lei, A. Z-Selective Addition of Diaryl Phosphine Oxides to Alkynes Via Photoredox Catalysis. ACS Catal. 2018, 8, 1059910605.

(5) Pramanik, M.; Choudhuri, K.; Mathuri, A.; Mal, P. Dithioacetalization or Thioetherification of Benzyl Alcohols Using 9-Mesityl-10-Methylacridinium Perchlorate Photocatalyst. Chem. Commun. 2020, 56, 10211-10214.

(6) SAINT+, Bruker AXS Inc., Madison, Wisconsin, USA, 1999 (Program for Reduction of Data collected on Bruker CCD Area Detector Diffractometer V. 6.02.)

(7) Sheldrick, G. In Sadabs, Program for Empirical Absorption Correction of Area Detector Data, 1996.

(8) Sheldrick, G. A Short History of Shelx. Acta Crystallogr. Sect. A 2008, 64, 112-122.

(9) Gao, W.-C.; Liu, T.; Zhang, B.; Li, X.; Wei, W.-L.; Liu, Q.; Tian, J.; Chang, H.-H. Synthesis of 3-Sulfenylated Coumarins: $\mathrm{BF}_{3} \cdot \mathrm{Et}_{2} \mathrm{O}$-Mediated Electrophilic Cyclization of Aryl Alkynoates Using N-Sulfanylsuccinimides. J. Org. Chem. 2016, 81, 11297-11304. 
(10) Chen, L.; Wu, L.; Duan, W.; Wang, T.; Li, L.; Zhang, K.; Zhu, J.; Peng, Z.; Xiong, F. Photoredox-Catalyzed Cascade Radical Cyclization of Ester Arylpropiolates with $\mathrm{CF}_{3} \mathrm{SO}_{2} \mathrm{Cl}$ to Construct 3-Trifluoromethyl Coumarin Derivatives. J. Org. Chem. 2018, 83, 8607-8614.

(11) Mou, C.; Wu, J.; Huang, Z.; Sun, J.; Jin, Z.; Chi, Y. R. Carbene-Catalyzed Lumo Activation of Alkyne Esters for Access to Functional Pyridines. Chem. Commun. 2017, 53, 13359-13362.

(12) Wang, Z.; Li, X.; Wang, L.; Li, P. Photoinduced Cyclization of Alkynoates to Coumarins with N-Iodosuccinimide as a Free-Radical Initiator under Ambient And metal-Free Conditions. Tetrahedron 2019, 75, 1044-1051.

(13) Liu, D.; Chen, J.-Q.; Wang, X.-Z.; Xu, P.-F. Metal-Free, Visible-Light-Promoted Synthesis of 3-Phosphorylated Coumarins Via Radical C-P/C-C Bond Formation. Adv. Synth. Catal. 2017, 359, 2773-2777.

(14) Li, H.; Liu, S.; Huang, Y.; Xu, X.-H.; Qing, F.-L. Tandem Trifluoromethylthiolation/Aryl Migration of Aryl Alkynoates to Trifluoromethylthiolated Alkenes. Chem. Commun. 2017, 53, 10136-10139. 

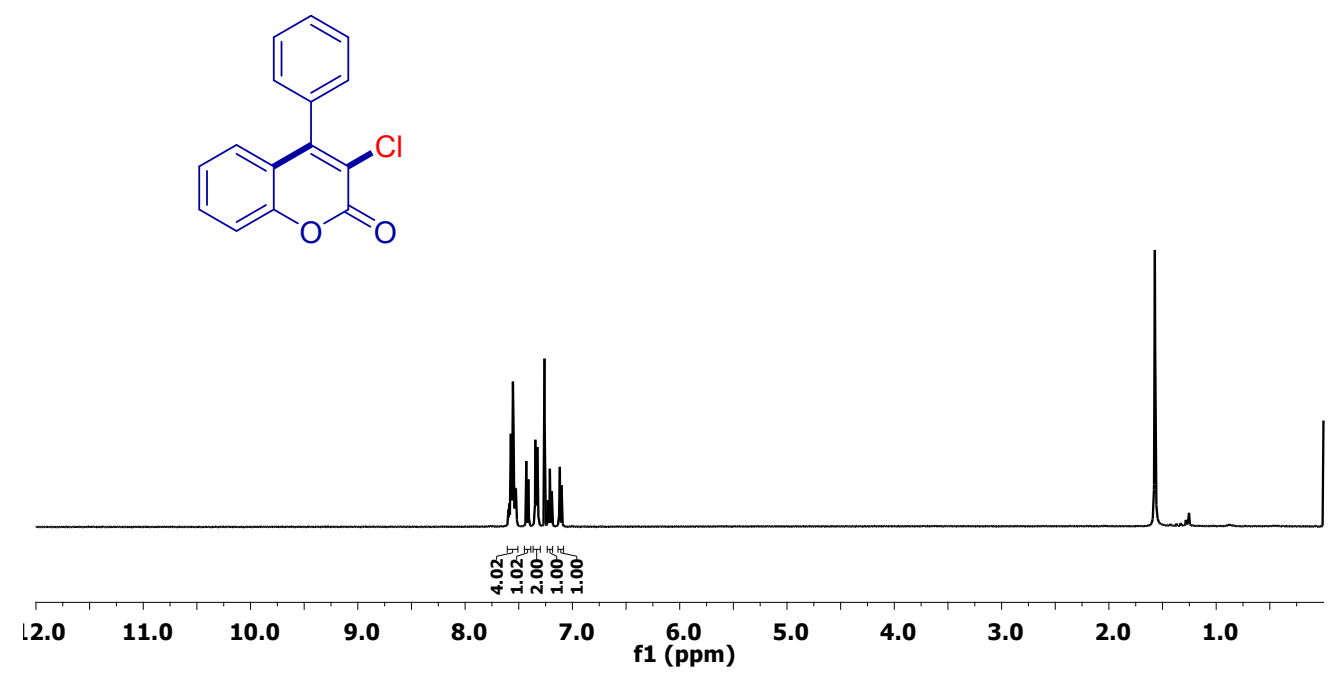

Fig. S7. ${ }^{1} \mathrm{H}$ NMR (400 MHz, $\mathrm{CDCl}_{3}$ ) spectrum of 3-chloro-4-phenyl-2H-chromen-2-one (2a).

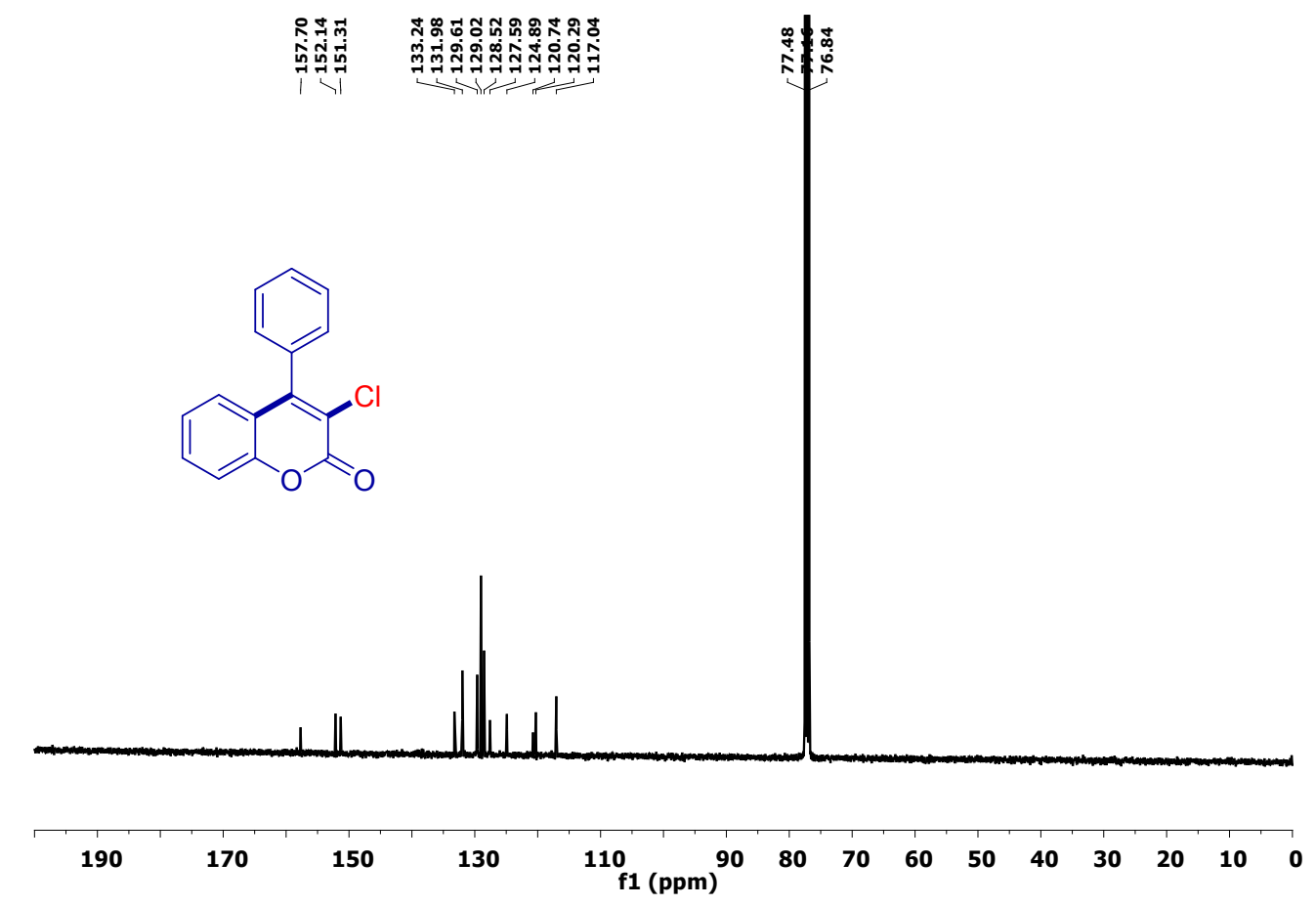

Fig. S8. ${ }^{13} \mathrm{C} \mathrm{NMR}\left(100 \mathrm{MHz}, \mathrm{CDCl}_{3}\right)$ spectrum of 3-chloro-4-phenyl-2H-chromen-2-one (2a). 


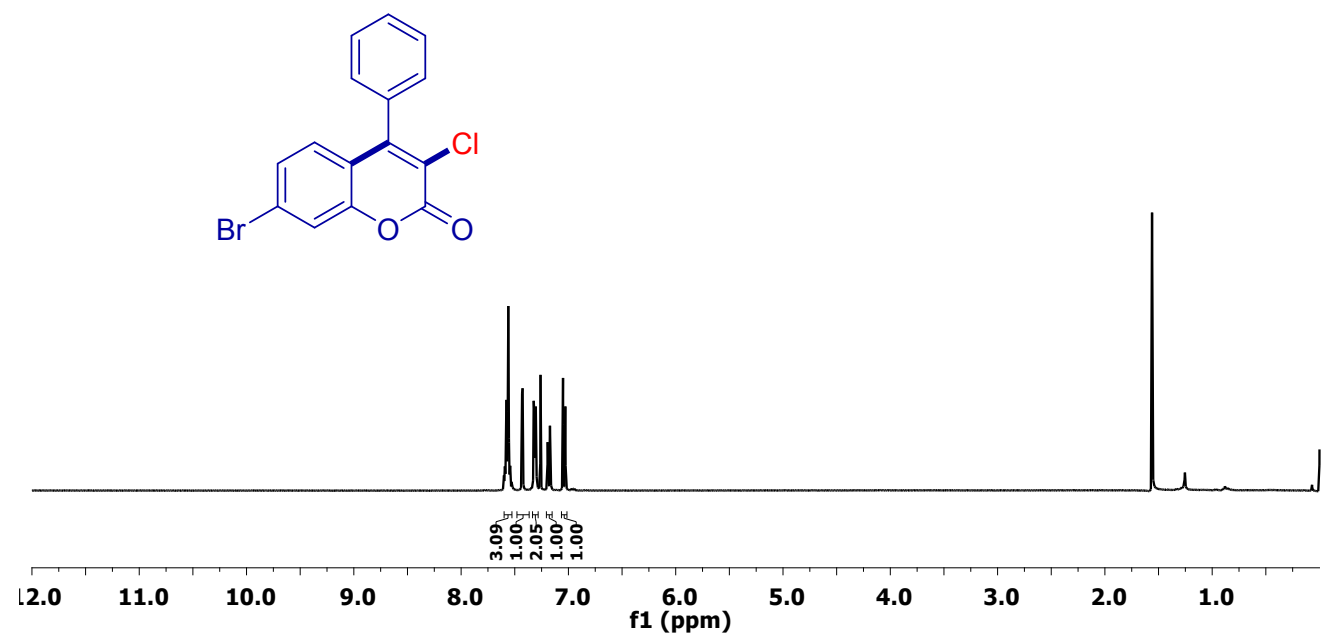

Fig. S9. ${ }^{1} \mathrm{H} \mathrm{NMR}\left(400 \mathrm{MHz}, \mathrm{CDCl}_{3}\right)$ spectrum of 7-bromo-3-chloro-4-phenyl-2H-chromen-2one (2b).

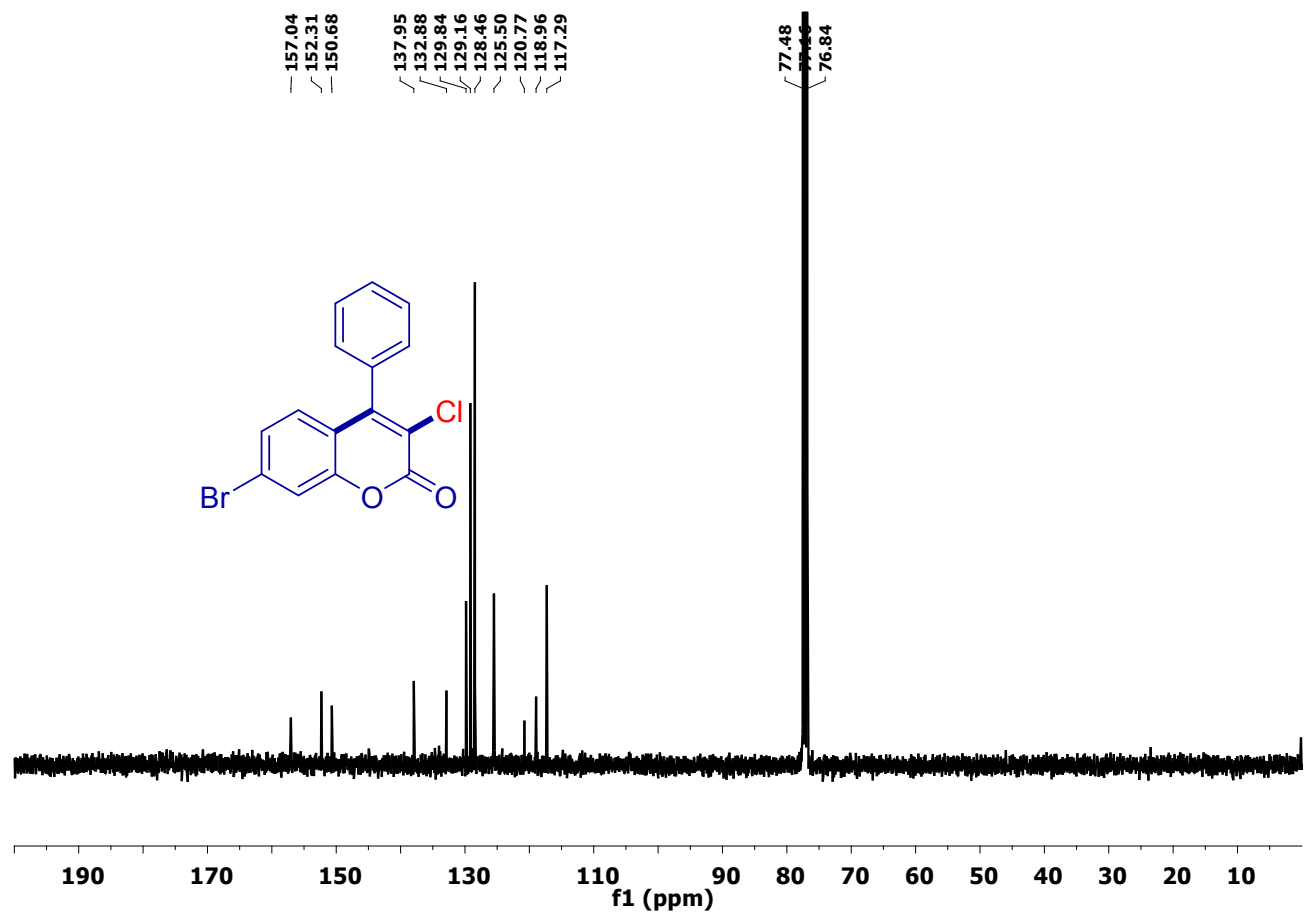

Fig. S10. ${ }^{13} \mathrm{C}$ NMR (100 MHz, $\left.\mathrm{CDCl}_{3}\right)$ spectrum of 7-bromo-3-chloro-4-phenyl-2H-chromen-2one (2b). 

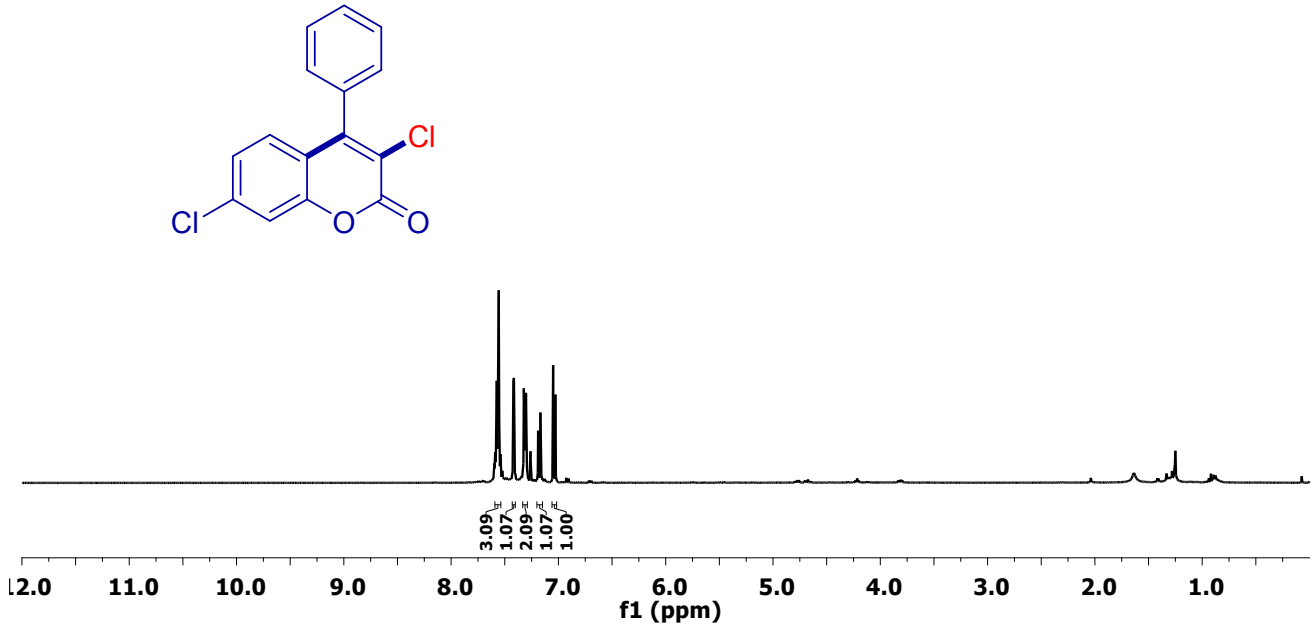

Fig.11. ${ }^{1} \mathrm{H} \mathrm{NMR}\left(400 \mathrm{MHz}, \mathrm{CDCl}_{3}\right)$ spectrum of 3,7-dichloro-4-phenyl-2H-chromen-2-one (2c).
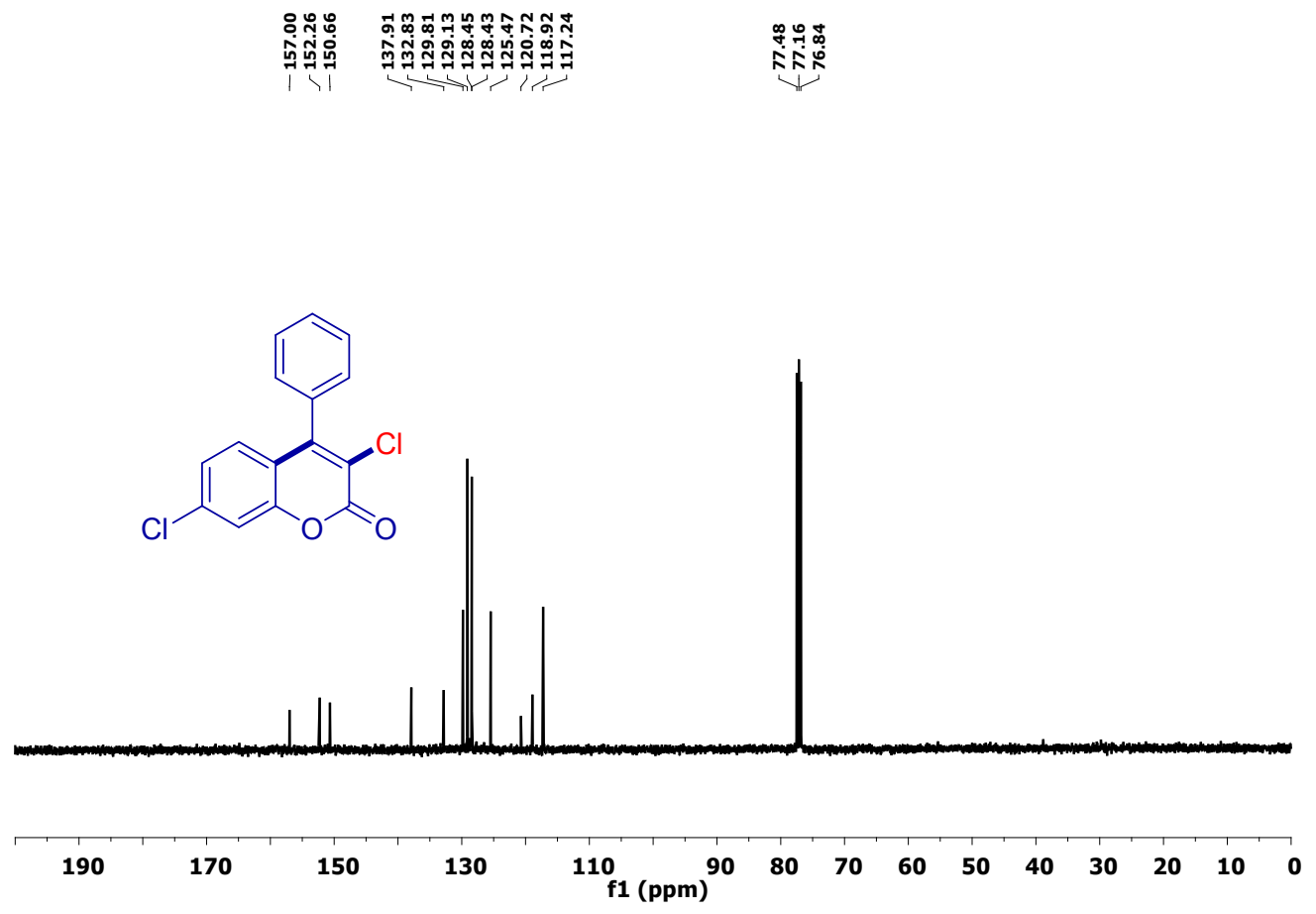

Fig. S12. ${ }^{13} \mathrm{C}$ NMR $\left(100 \mathrm{MHz}, \mathrm{CDCl}_{3}\right)$ spectrum of 3,7-dichloro-4-phenyl-2H-chromen-2-one (2c). 


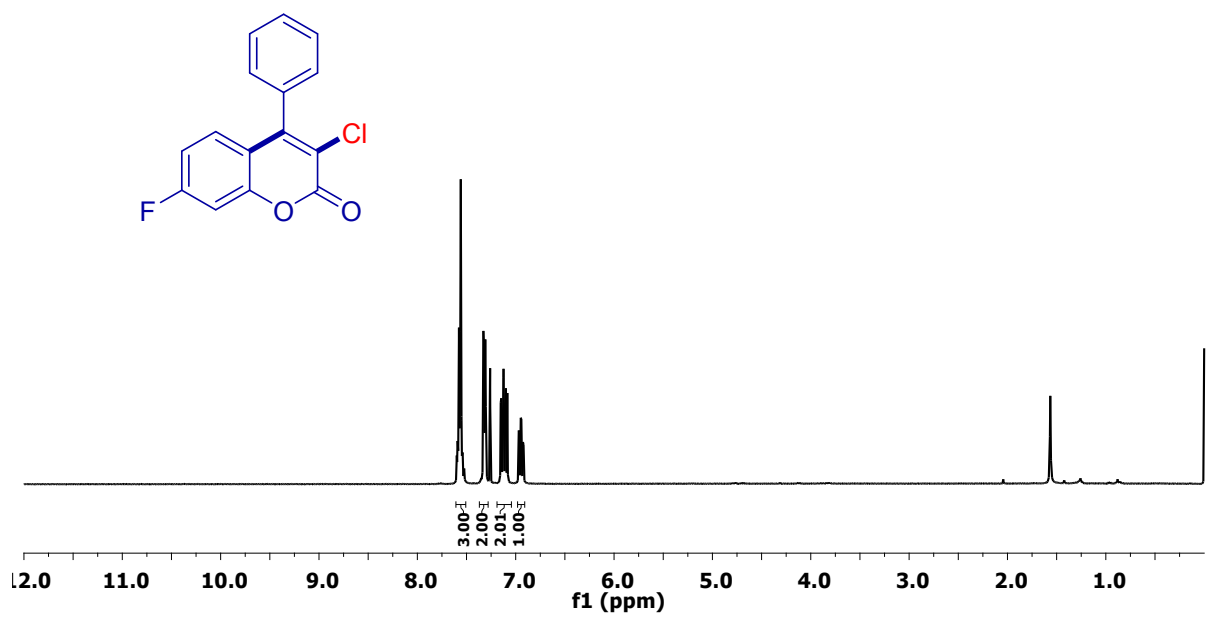

Fig.13. ${ }^{1} \mathrm{H} \mathrm{NMR}\left(400 \mathrm{MHz}, \mathrm{CDCl}_{3}\right)$ spectrum of 3-chloro-7-fluoro-4-phenyl-2H-chromen-2-one (2d).

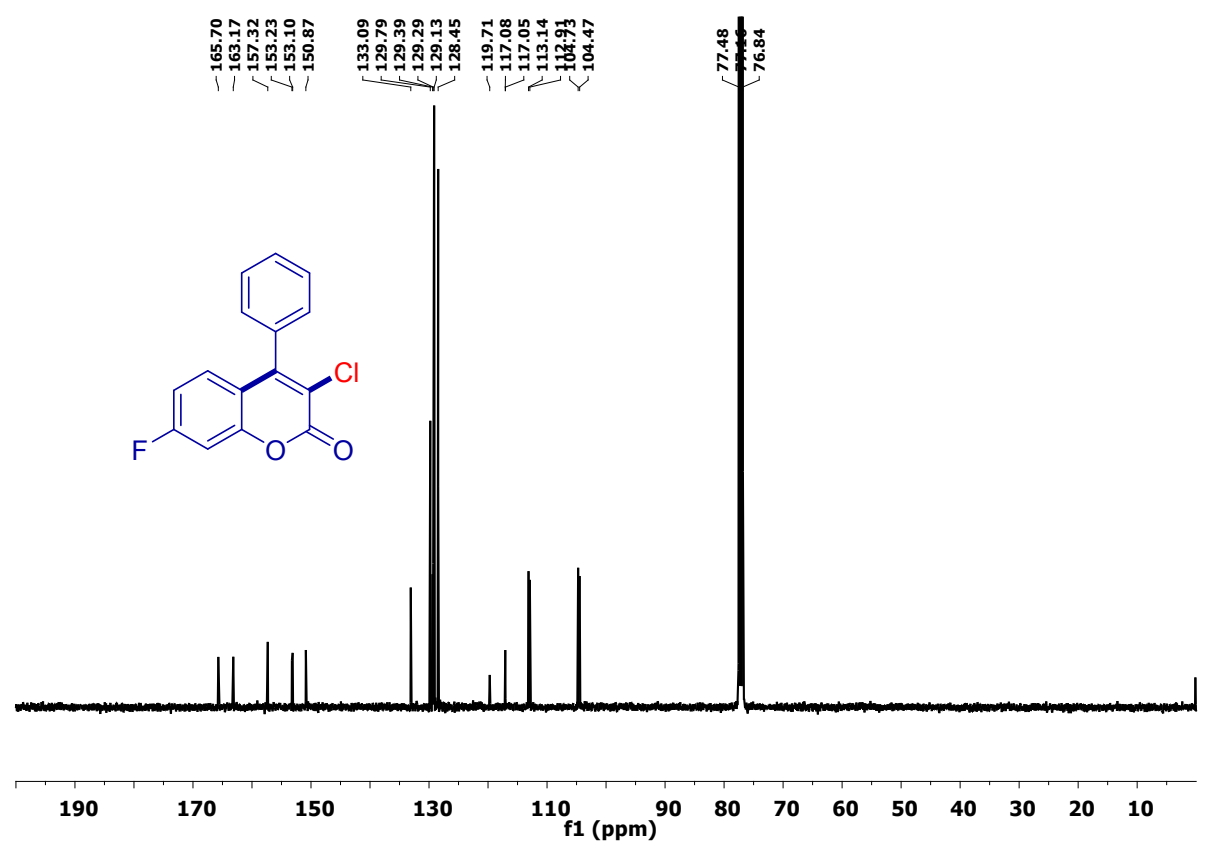

Fig. S14. ${ }^{13} \mathrm{C}$ NMR (100 MHz, $\mathrm{CDCl}_{3}$ ) spectrum of 3-chloro-7-fluoro-4-phenyl-2H-chromen-2one (2d). 


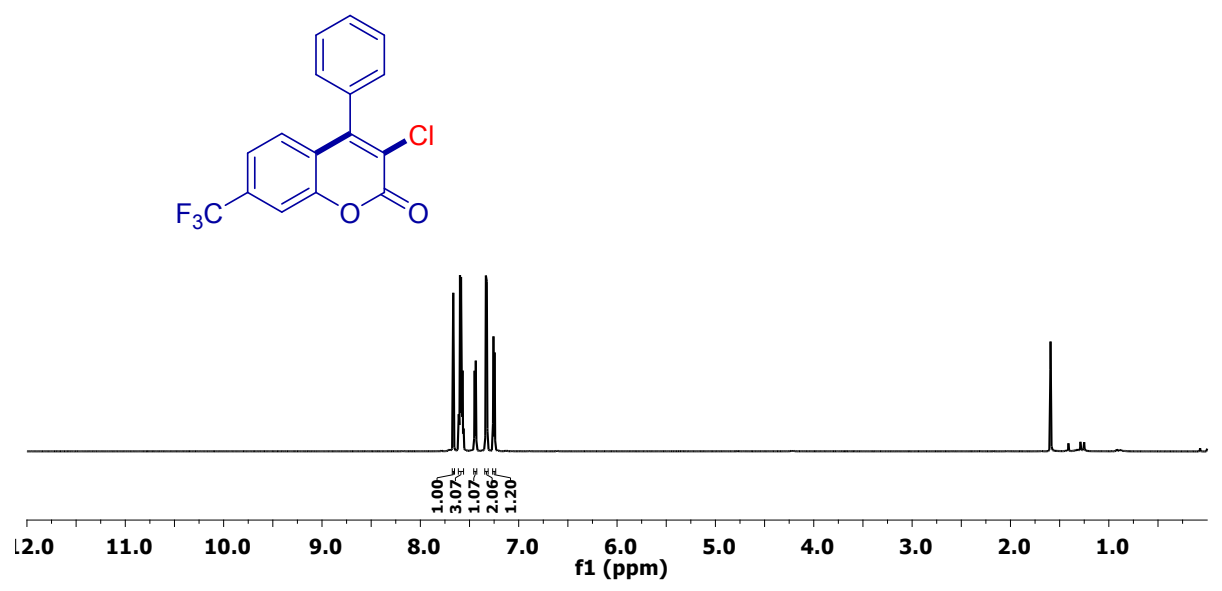

Fig.15. ${ }^{1} \mathrm{H} \mathrm{NMR}\left(700 \mathrm{MHz}, \mathrm{CDCl}_{3}\right)$ spectrum of 3-chloro-4-phenyl-7-(trifluoromethyl)-2Hchromen-2-one (2e).

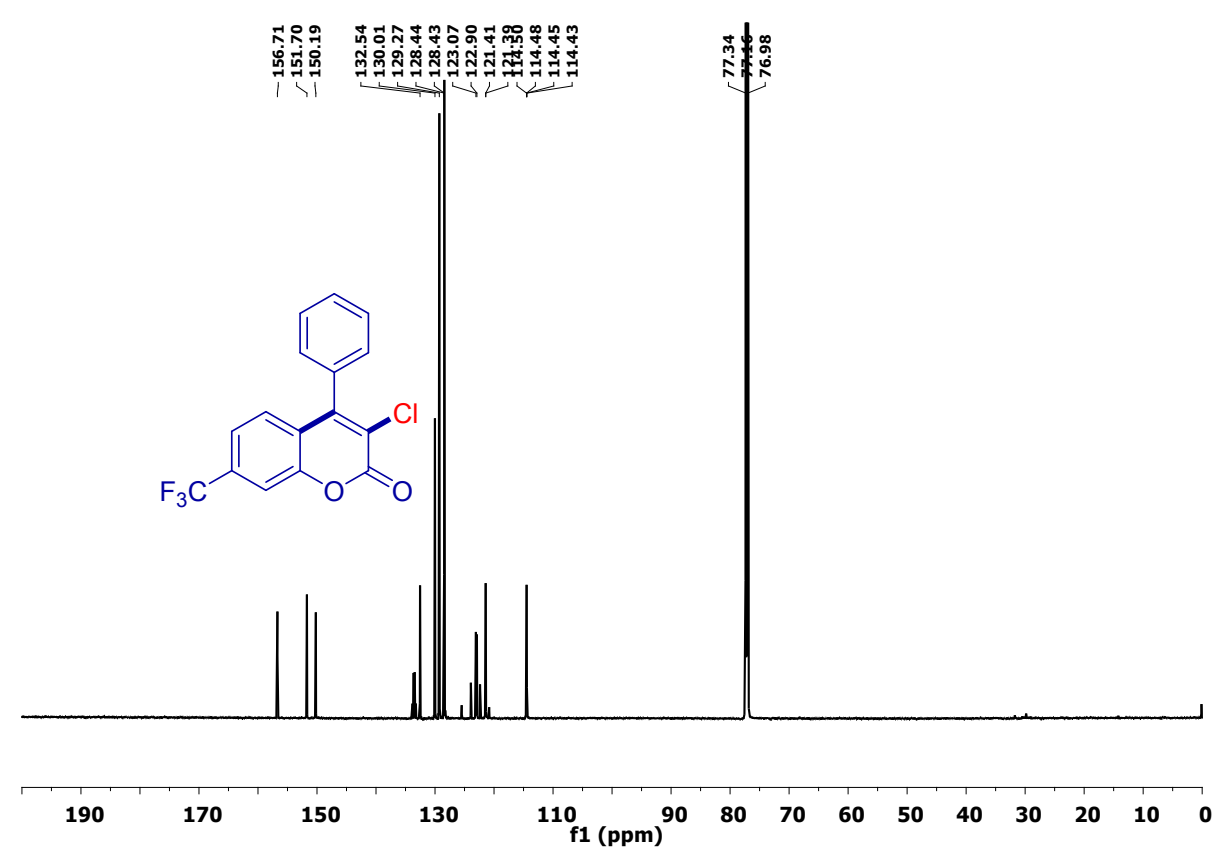

Fig. S16. ${ }^{13} \mathrm{C} \mathrm{NMR}\left(175 \mathrm{MHz}, \mathrm{CDCl}_{3}\right)$ spectrum of 3-chloro-4-phenyl-7-(trifluoromethyl)-2Hchromen-2-one (2e). 

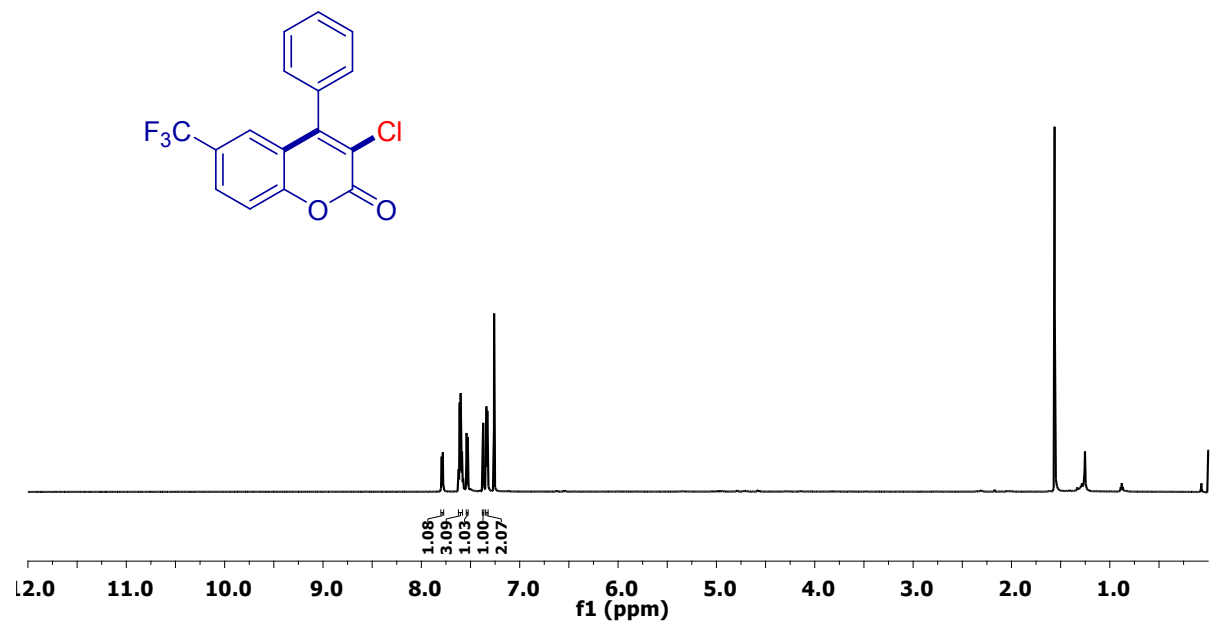

Fig. S17. ${ }^{1} \mathrm{H}$ NMR (700 MHz, $\mathrm{CDCl}_{3}$ ) spectrum of 3-chloro-4-phenyl-6-(trifluoromethyl)-2Hchromen-2-one (2f).

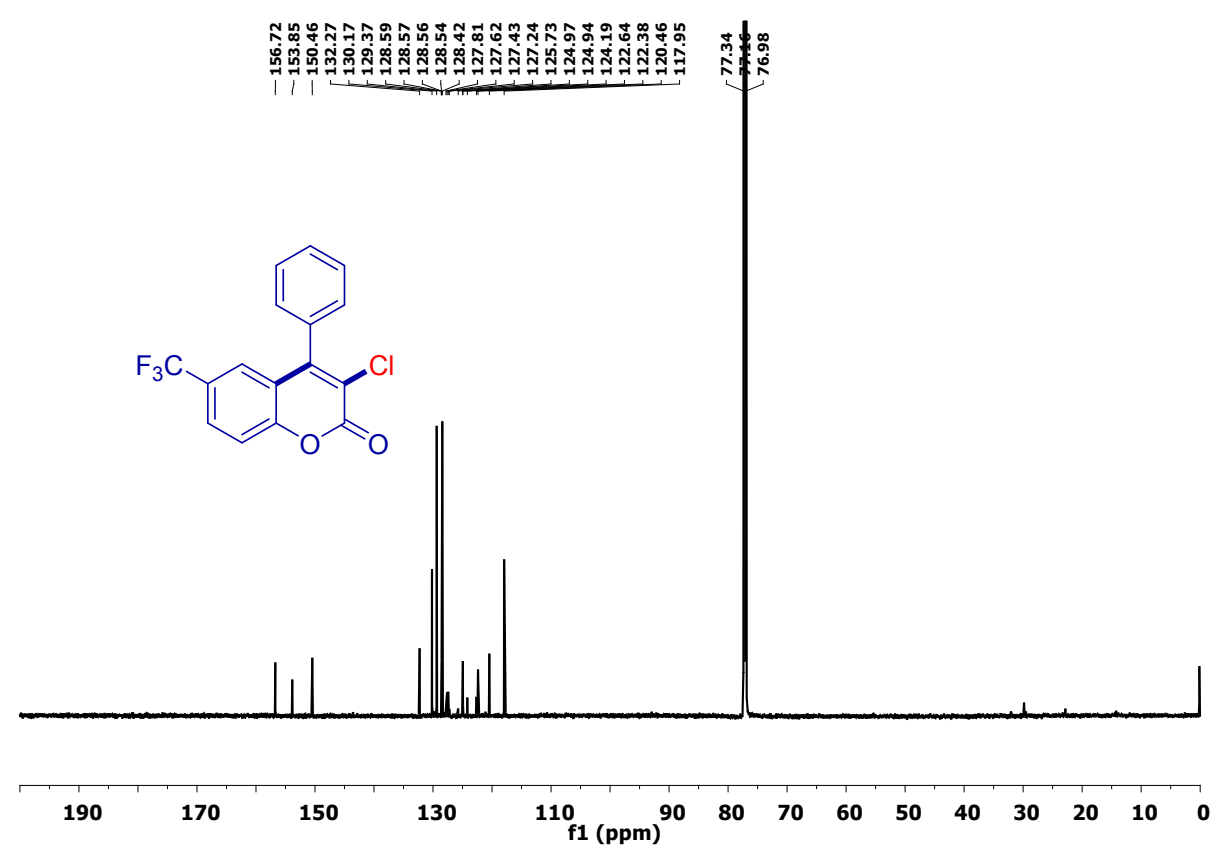

Fig. S18. ${ }^{13} \mathrm{C} \mathrm{NMR}\left(175 \mathrm{MHz}, \mathrm{CDCl}_{3}\right)$ spectrum of 3-chloro-4-phenyl-6-(trifluoromethyl)-2Hchromen-2-one (2f). 


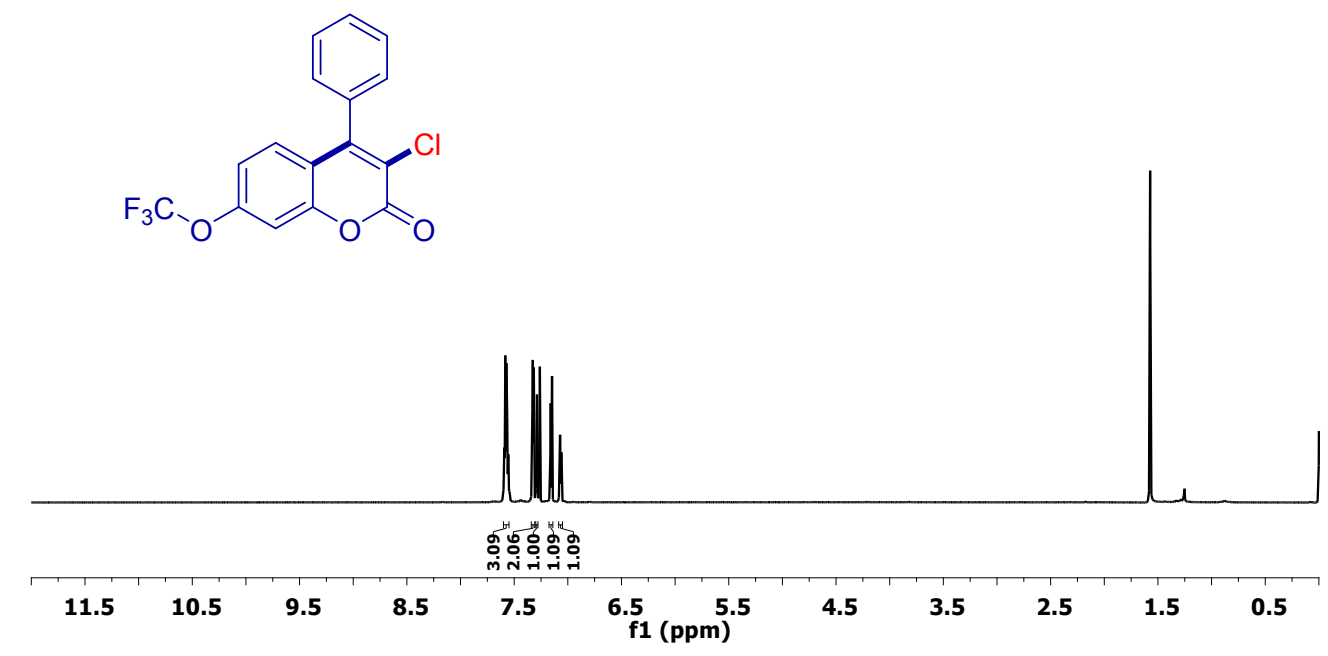

Fig. S19. ${ }^{1} \mathrm{H} \mathrm{NMR}\left(700 \mathrm{MHz}, \mathrm{CDCl}_{3}\right)$ spectrum of 3-chloro-4-phenyl-7-(trifluoromethoxy)-2Hchromen-2-one (2g).

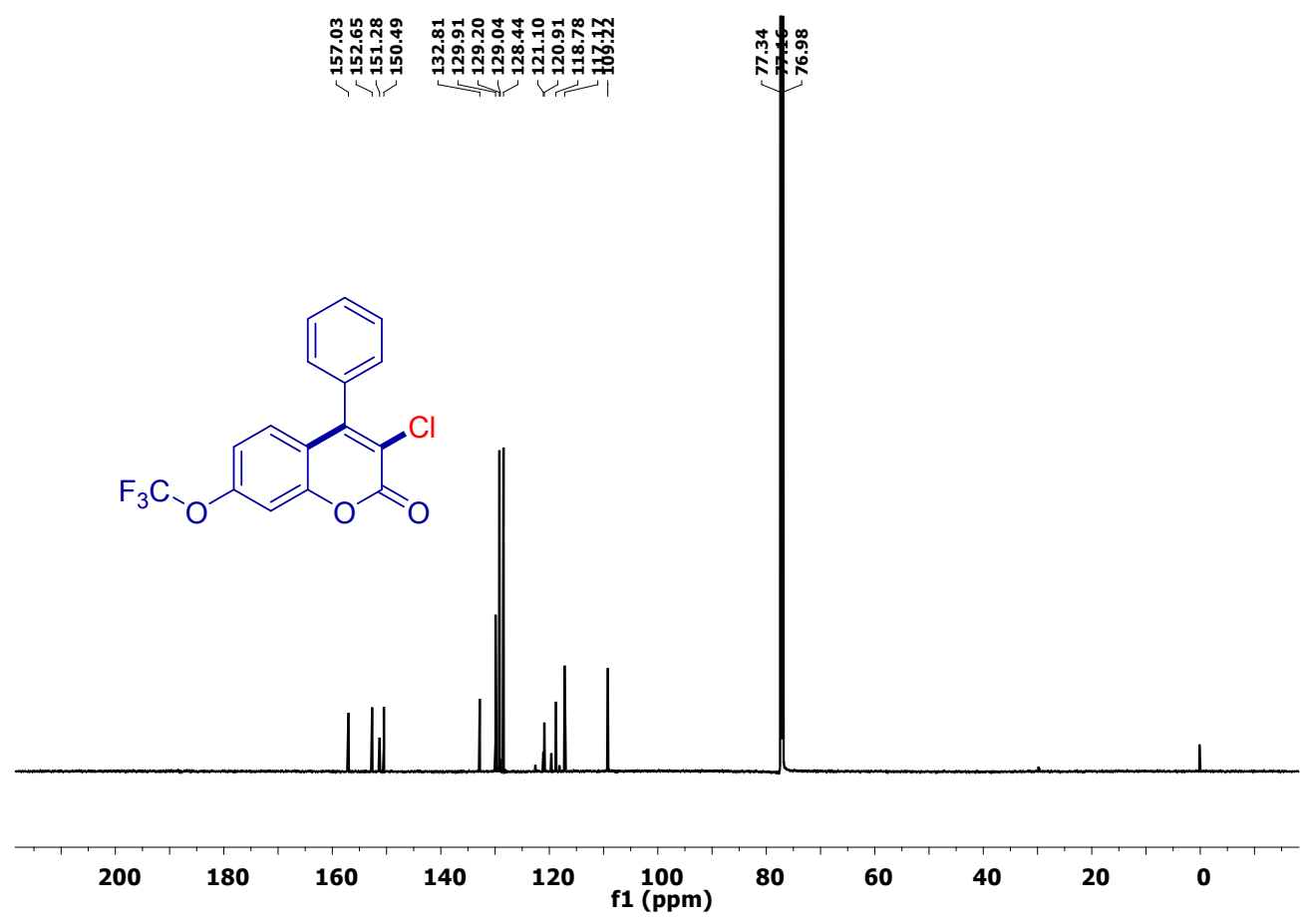

Fig. S20. ${ }^{13} \mathrm{C}$ NMR (100 MHz, $\mathrm{CDCl}_{3}$ ) spectrum of 3-chloro-4-phenyl-7-(trifluoromethoxy)-2Hchromen-2-one (2g). 


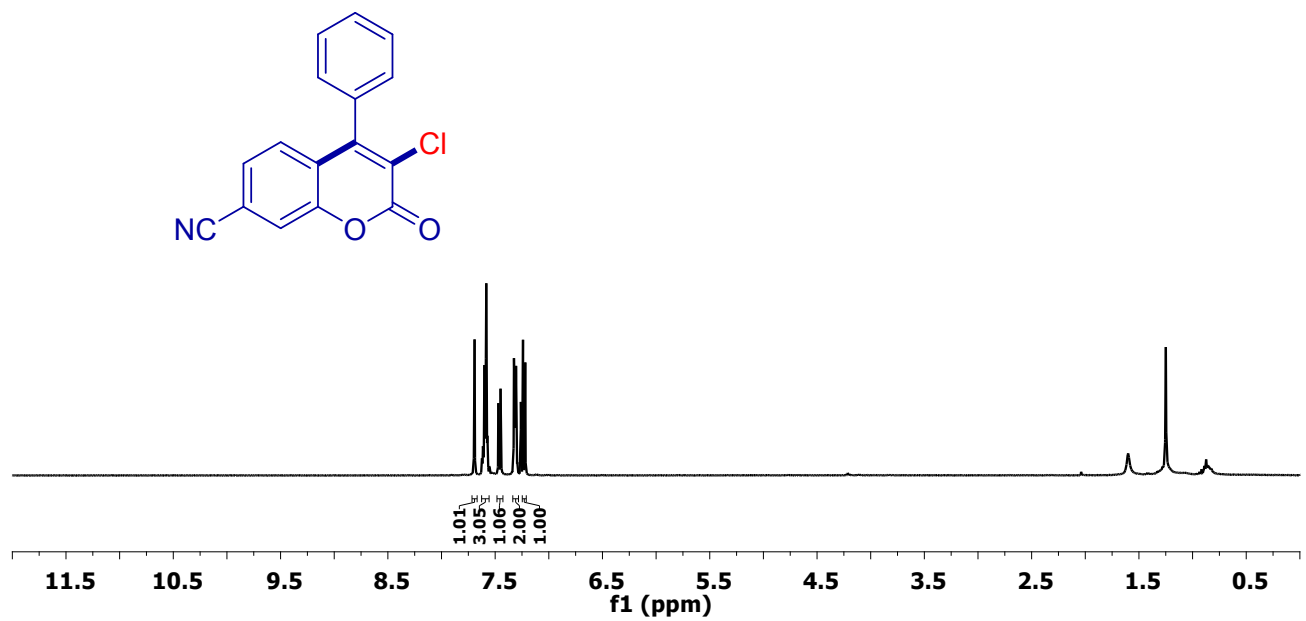

Fig. S21. ${ }^{1} \mathrm{H}$ NMR (400 MHz, $\mathrm{CDCl}_{3}$ ) spectrum of 3-chloro-2-oxo-4-phenyl-2H-chromene-7carbonitrile (2h).

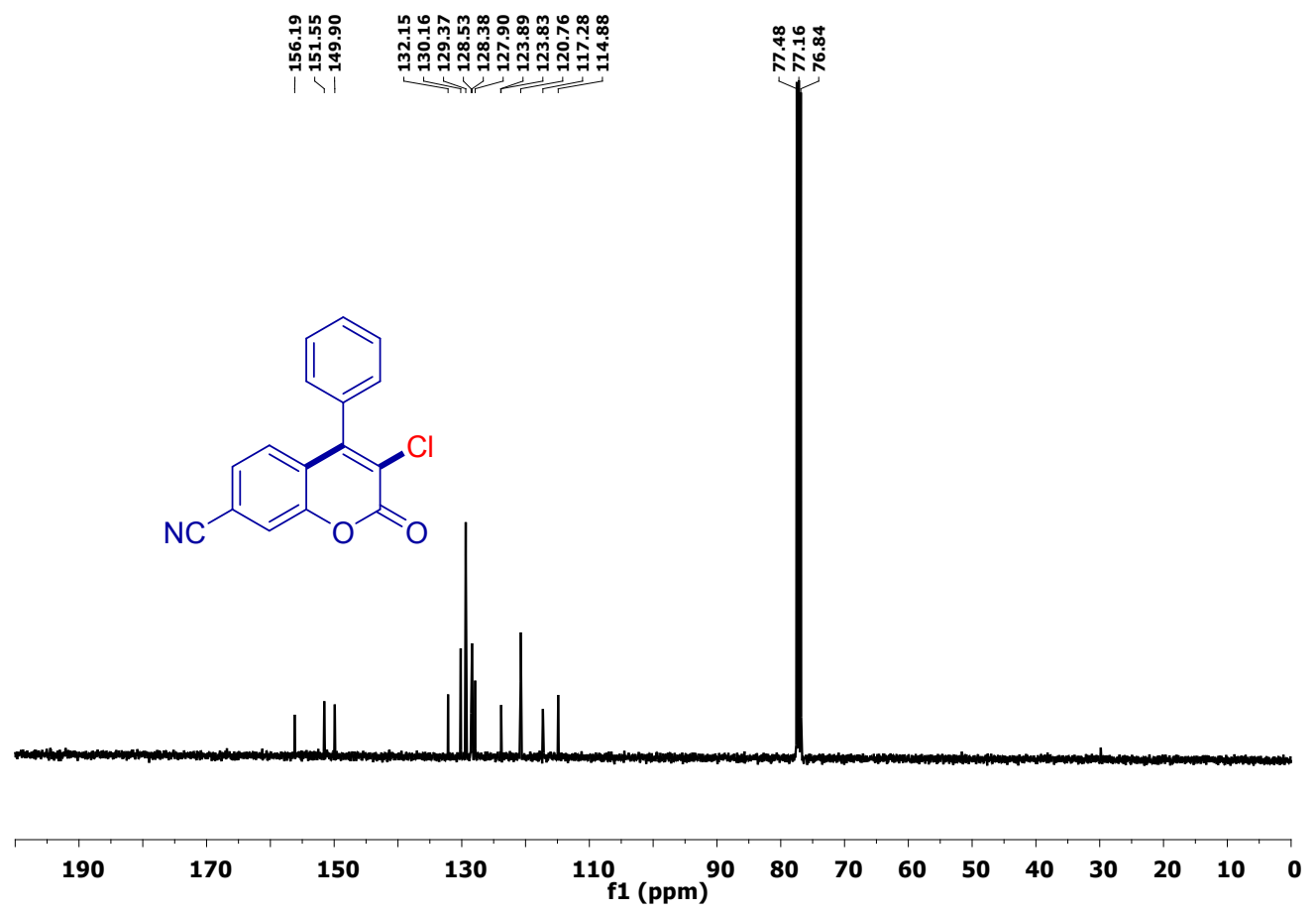

Fig. S22. ${ }^{13} \mathrm{C}$ NMR $\left(100 \mathrm{MHz}, \mathrm{CDCl}_{3}\right)$ spectrum of 3-chloro-2-oxo-4-phenyl-2H-chromene-7carbonitrile (2h). 

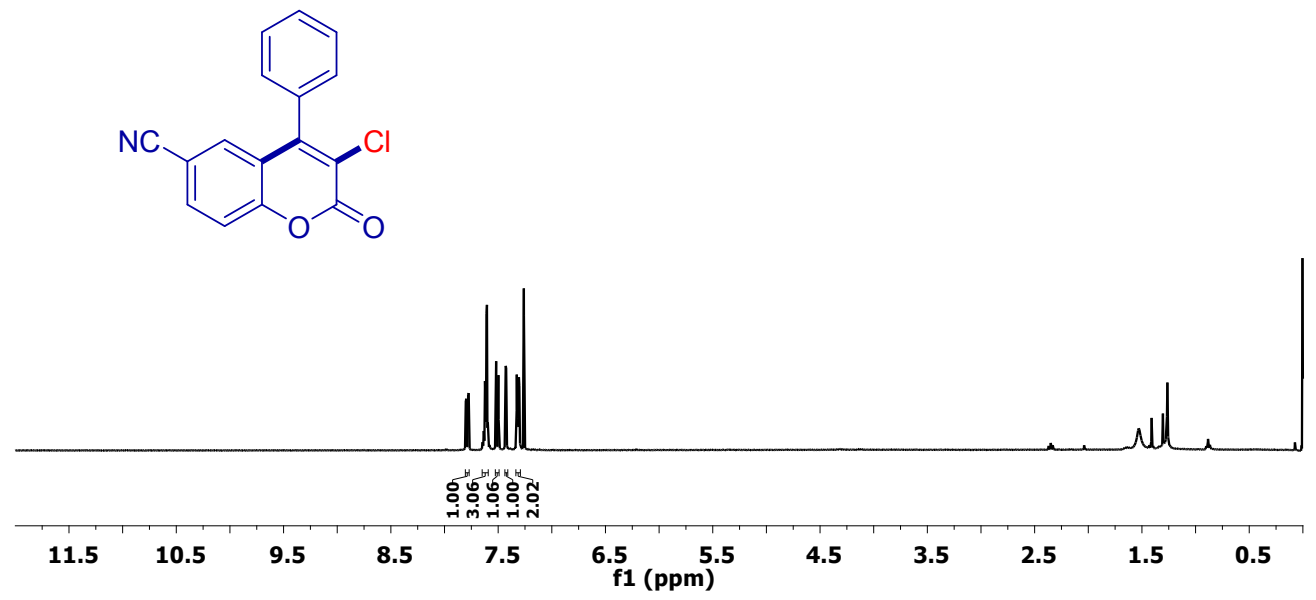

Fig. S23. ${ }^{1} \mathrm{H} \mathrm{NMR}\left(400 \mathrm{MHz}, \mathrm{CDCl}_{3}\right.$ ) spectrum of 3-chloro-2-oxo-4-phenyl-2H-chromene-6carbonitrile (2i).

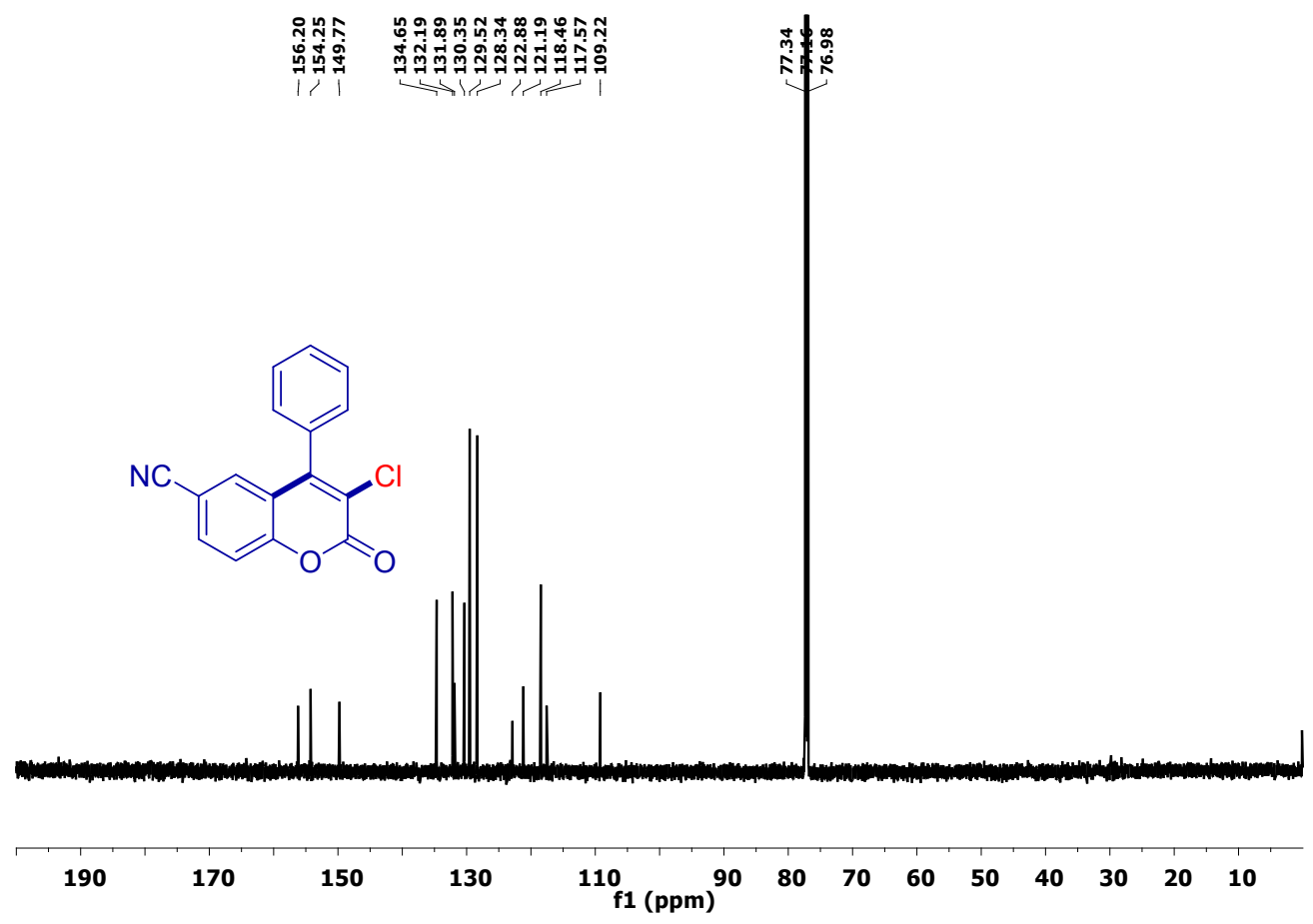

Fig. S24. ${ }^{13} \mathrm{C} \mathrm{NMR}\left(175 \mathrm{MHz}, \mathrm{CDCl}_{3}\right)$ spectrum of 3-chloro-2-oxo-4-phenyl-2H-chromene-6carbonitrile (2i). 

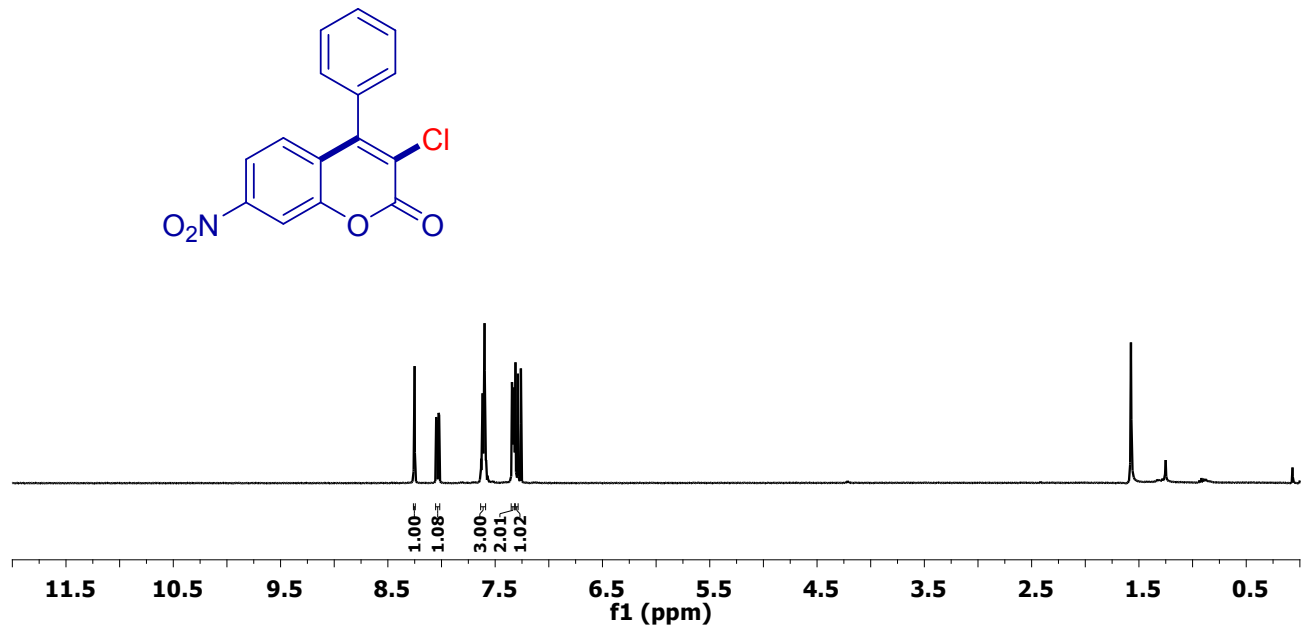

Fig. S25. ${ }^{1} \mathrm{H}$ NMR (400 MHz, $\left.\mathrm{CDCl}_{3}\right)$ spectrum of 3-chloro-7-nitro-4-phenyl-2H-chromen-2-one (2j).

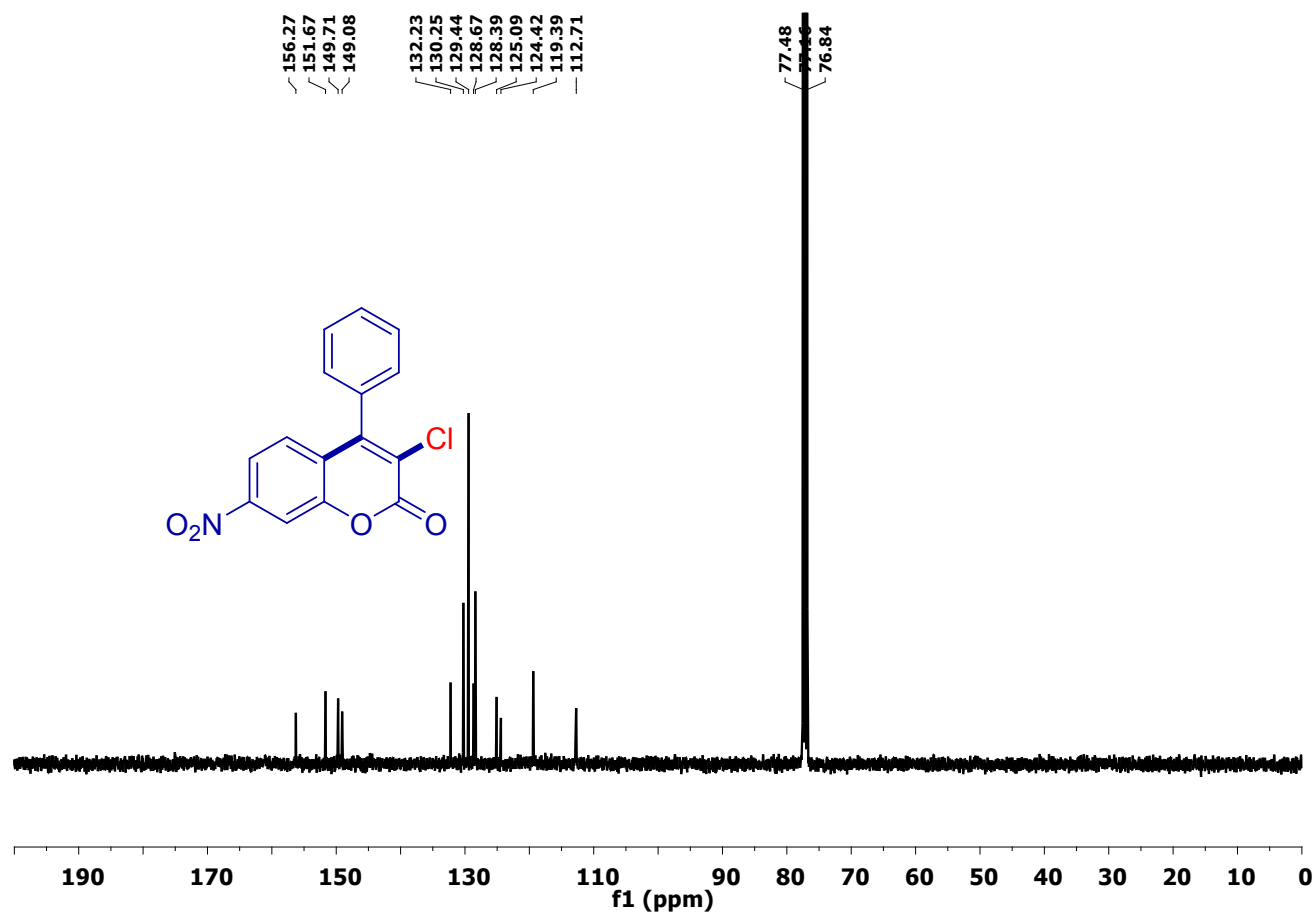

Fig. S26. ${ }^{13} \mathrm{C}$ NMR (100 MHz, $\left.\mathrm{CDCl}_{3}\right)$ spectrum of 3-chloro-7-nitro-4-phenyl-2H-chromen-2one $(\mathbf{2} \mathbf{j})$. 

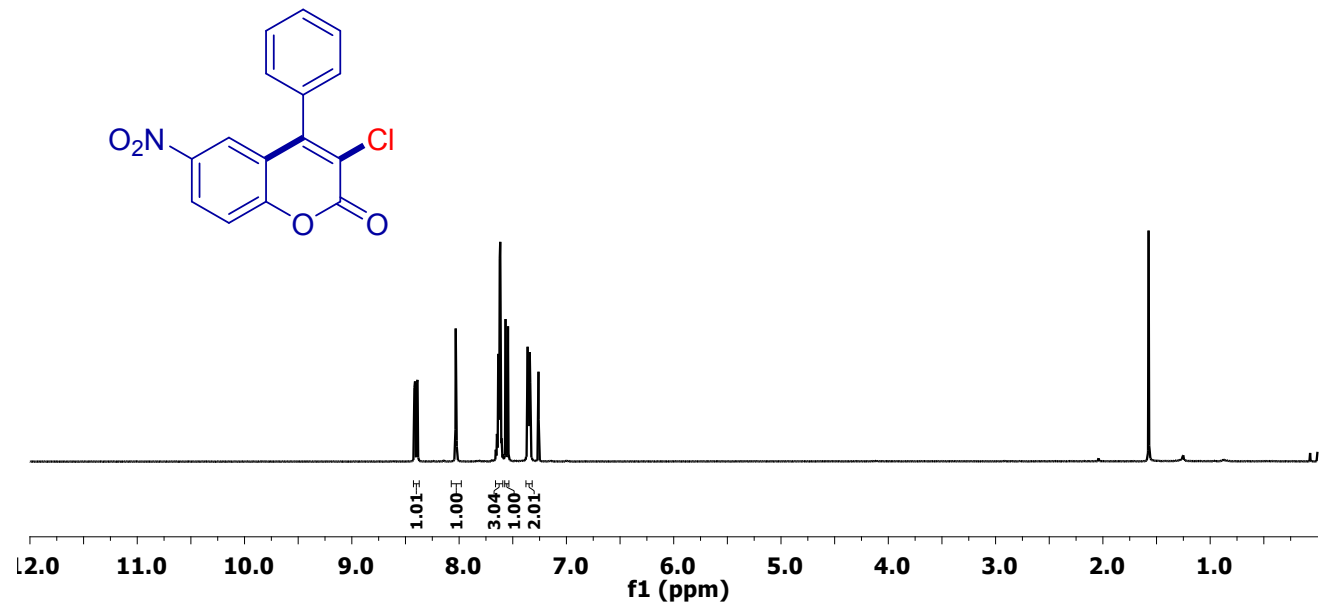

Fig. S27. ${ }^{1} \mathrm{H}$ NMR (400 MHz, $\left.\mathrm{CDCl}_{3}\right)$ spectrum of 3-chloro-6-nitro-4-phenyl-2H-chromen-2-one (2k).

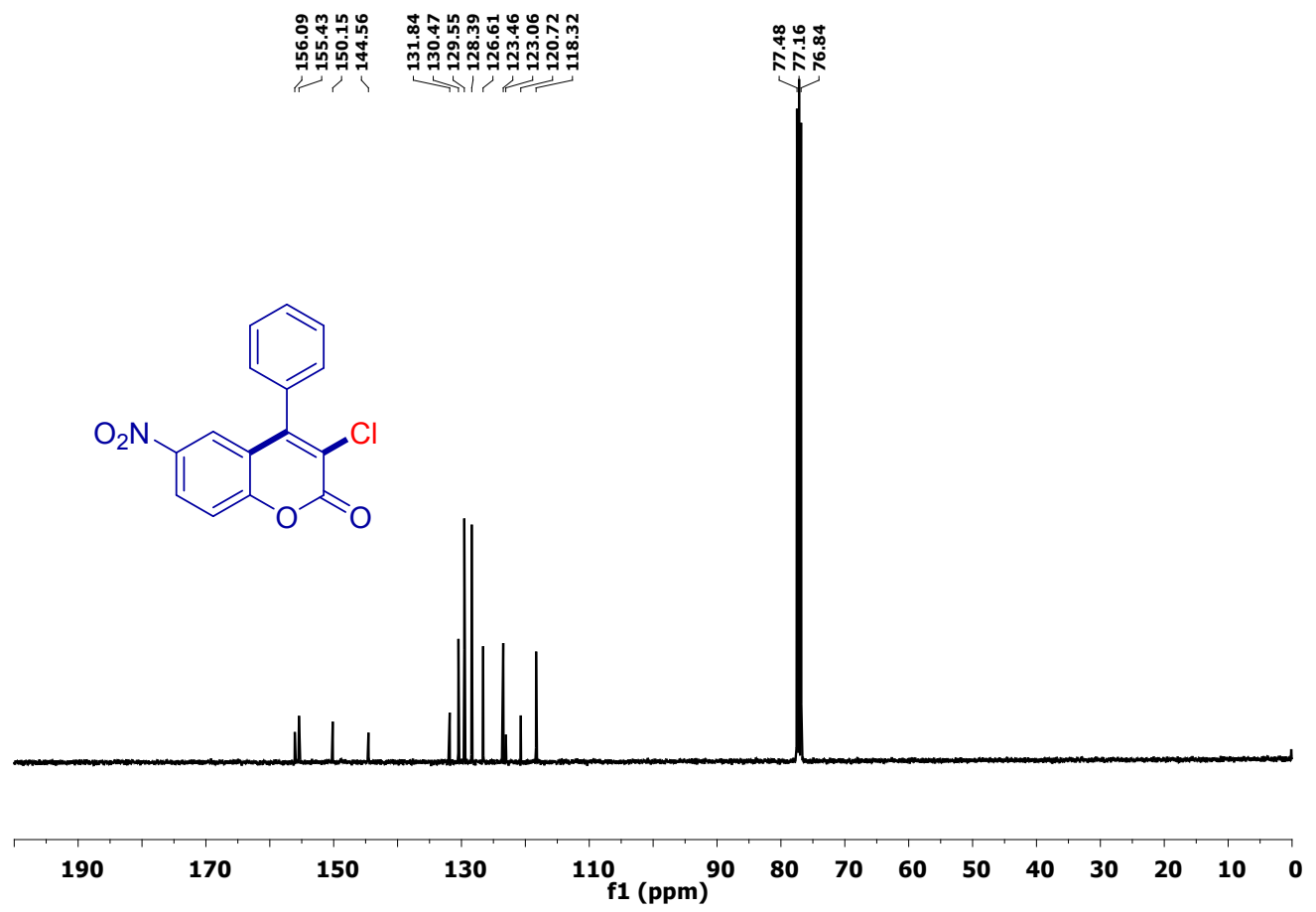

Fig. S28. ${ }^{13} \mathrm{C}$ NMR $\left(100 \mathrm{MHz}, \mathrm{CDCl}_{3}\right)$ spectrum of 3-chloro-6-nitro-4-phenyl-2H-chromen-2one (2k). 

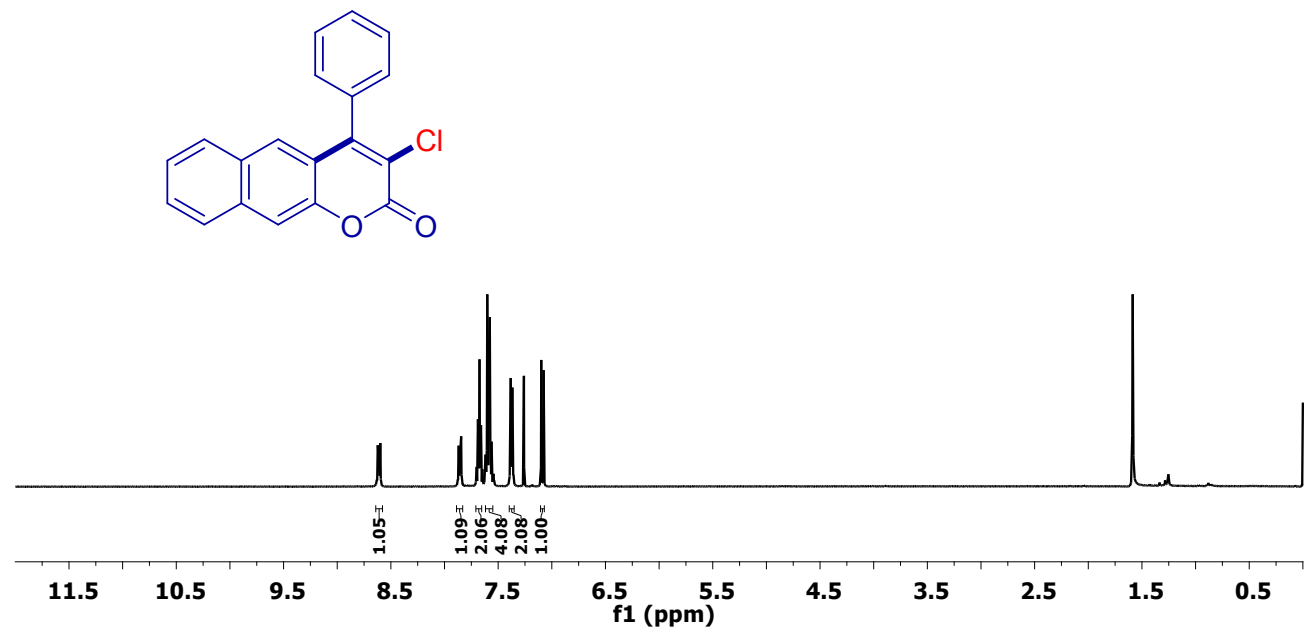

Fig. S29. ${ }^{1} \mathrm{H} \mathrm{NMR}\left(400 \mathrm{MHz}, \mathrm{CDCl}_{3}\right)$ spectrum of 3-chloro-4-phenyl-2H-benzo[g]chromen-2one (2l).

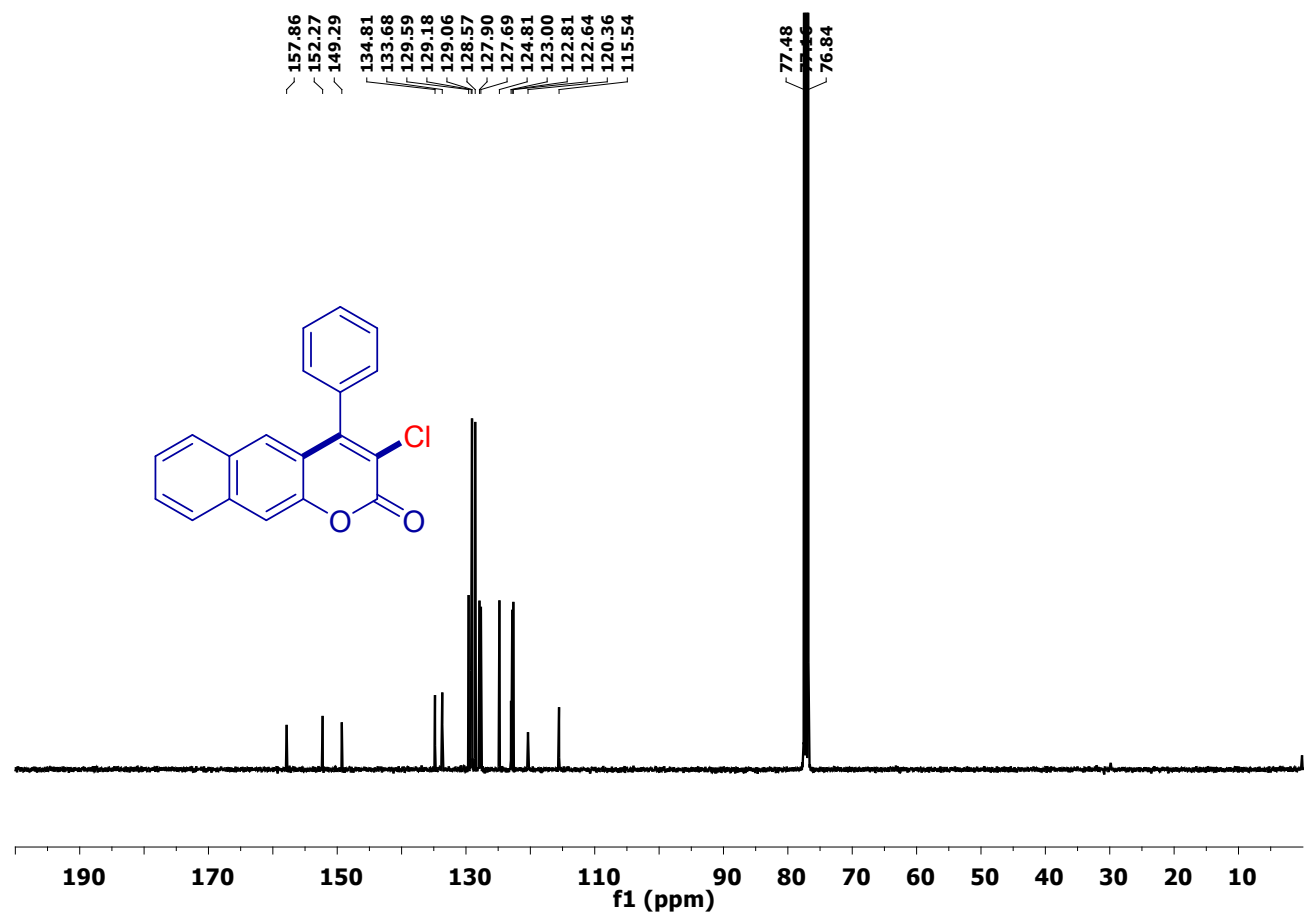

Fig. S30. ${ }^{13} \mathrm{C}$ NMR (100 MHz, $\left.\mathrm{CDCl}_{3}\right)$ spectrum of 3-chloro-4-phenyl-2H-benzo[g]chromen-2one (2l). 
<smiles>O=c1oc2cc(-c3ccccc3)ccc2c(-c2ccccc2)c1Cl</smiles>

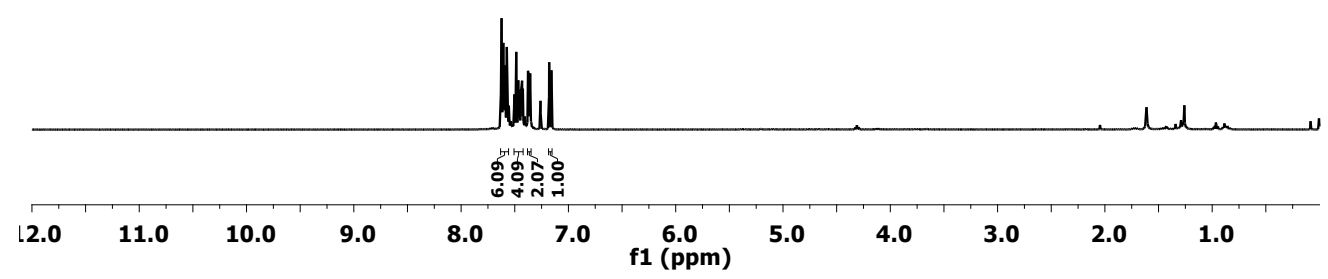

Fig. S31. ${ }^{1} \mathrm{H}$ NMR (400 MHz, $\mathrm{CDCl}_{3}$ ) spectrum of 3-chloro-4,7-diphenyl-2H-chromen-2-one (2m).
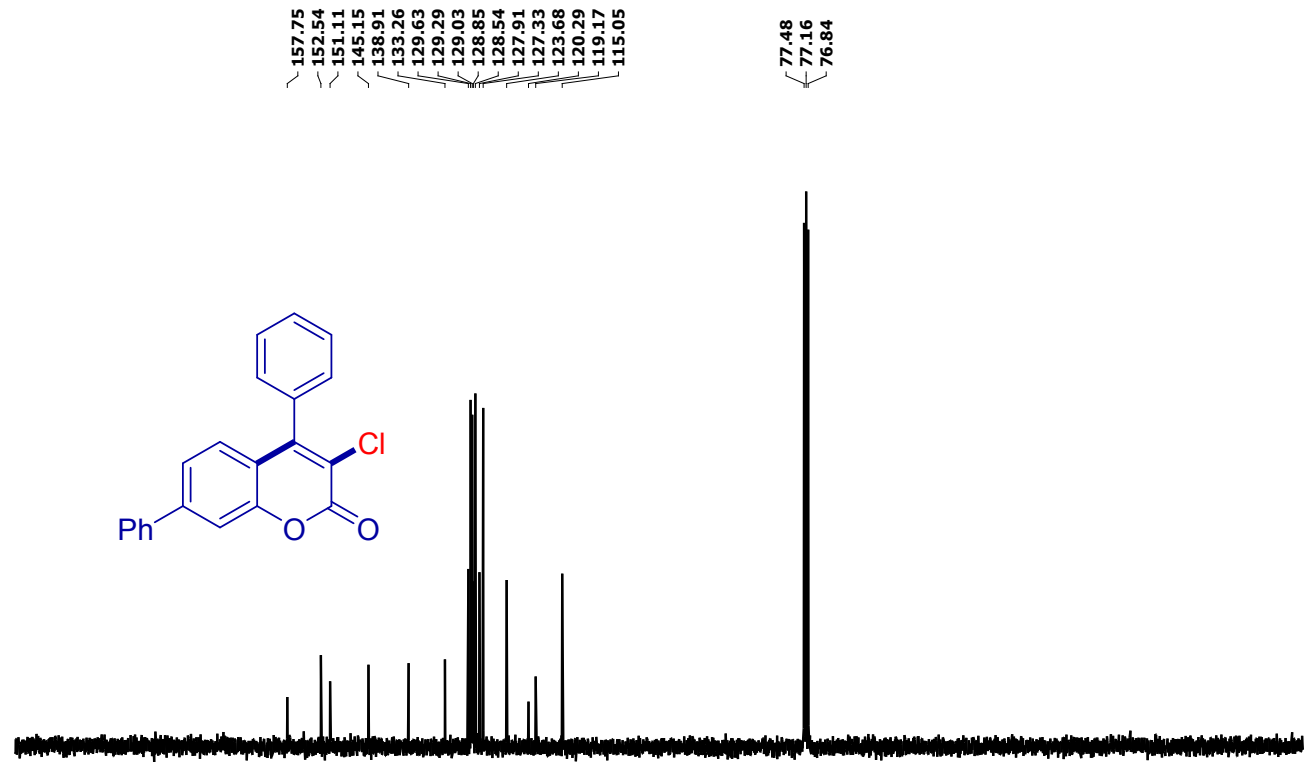

$190 \quad 170$

150

130

$\begin{array}{llllllllll}110 & 90 & 80 & 70 & 60 & 50 & 40 & 30 & 20 & 10\end{array}$

Fig. S32. ${ }^{13} \mathrm{C} \mathrm{NMR}\left(100 \mathrm{MHz}, \mathrm{CDCl}_{3}\right)$ spectrum of 3-chloro-4,7-diphenyl-2H-chromen-2-one (2m). 


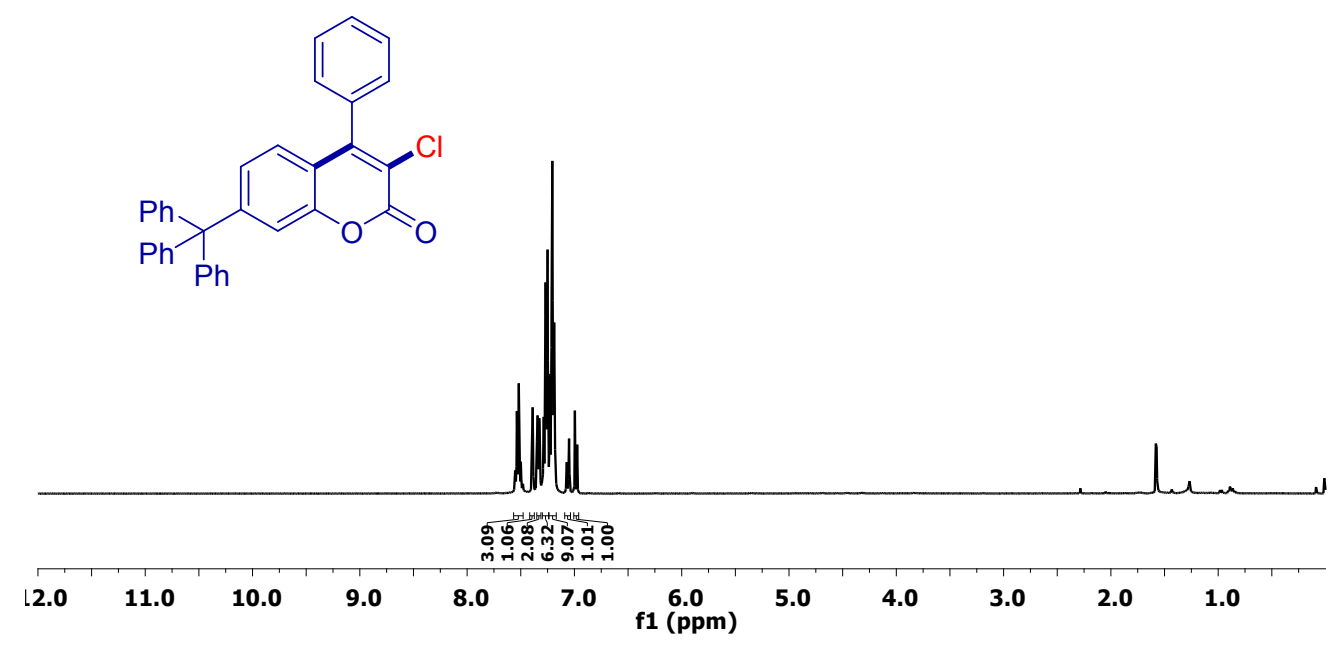

Fig. S33. ${ }^{1} \mathrm{H}$ NMR (400 MHz, $\mathrm{CDCl}_{3}$ ) spectrum of 3-chloro-4-phenyl-7-trityl-2H-chromen-2one (2n).

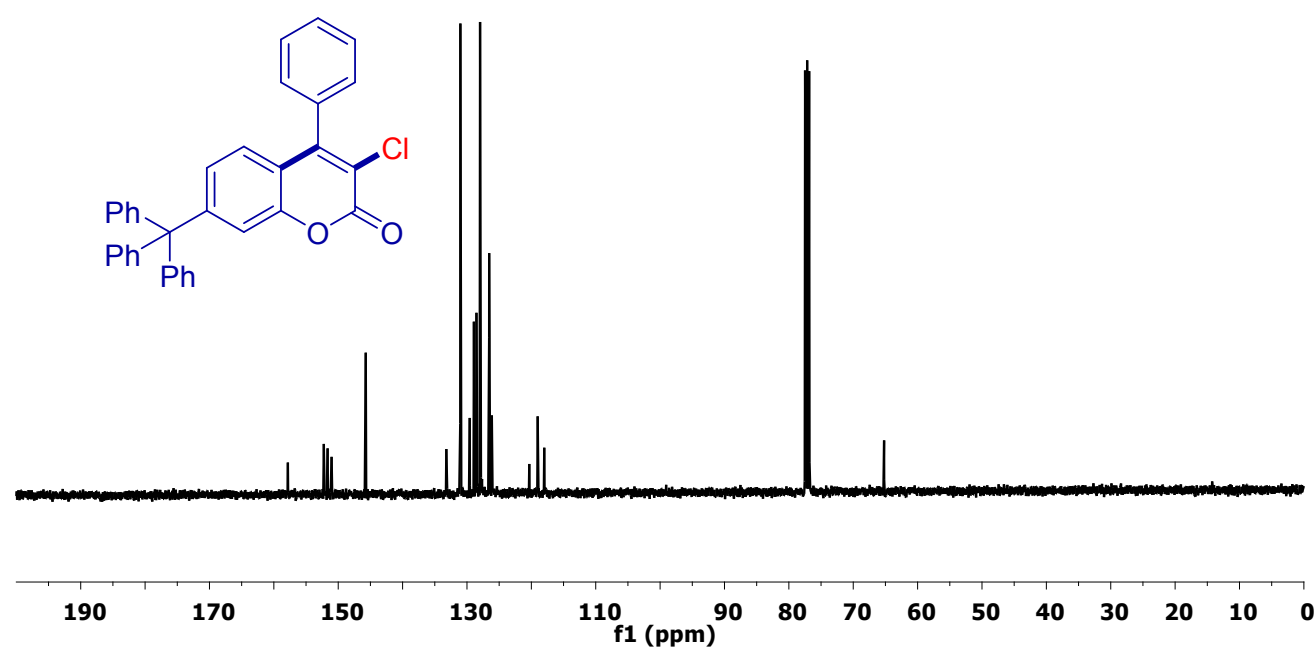

Fig. S34. ${ }^{13} \mathrm{C} \mathrm{NMR}\left(100 \mathrm{MHz}, \mathrm{CDCl}_{3}\right)$ spectrum of 3-chloro-4-phenyl-7-trityl-2H-chromen-2one (2n). 


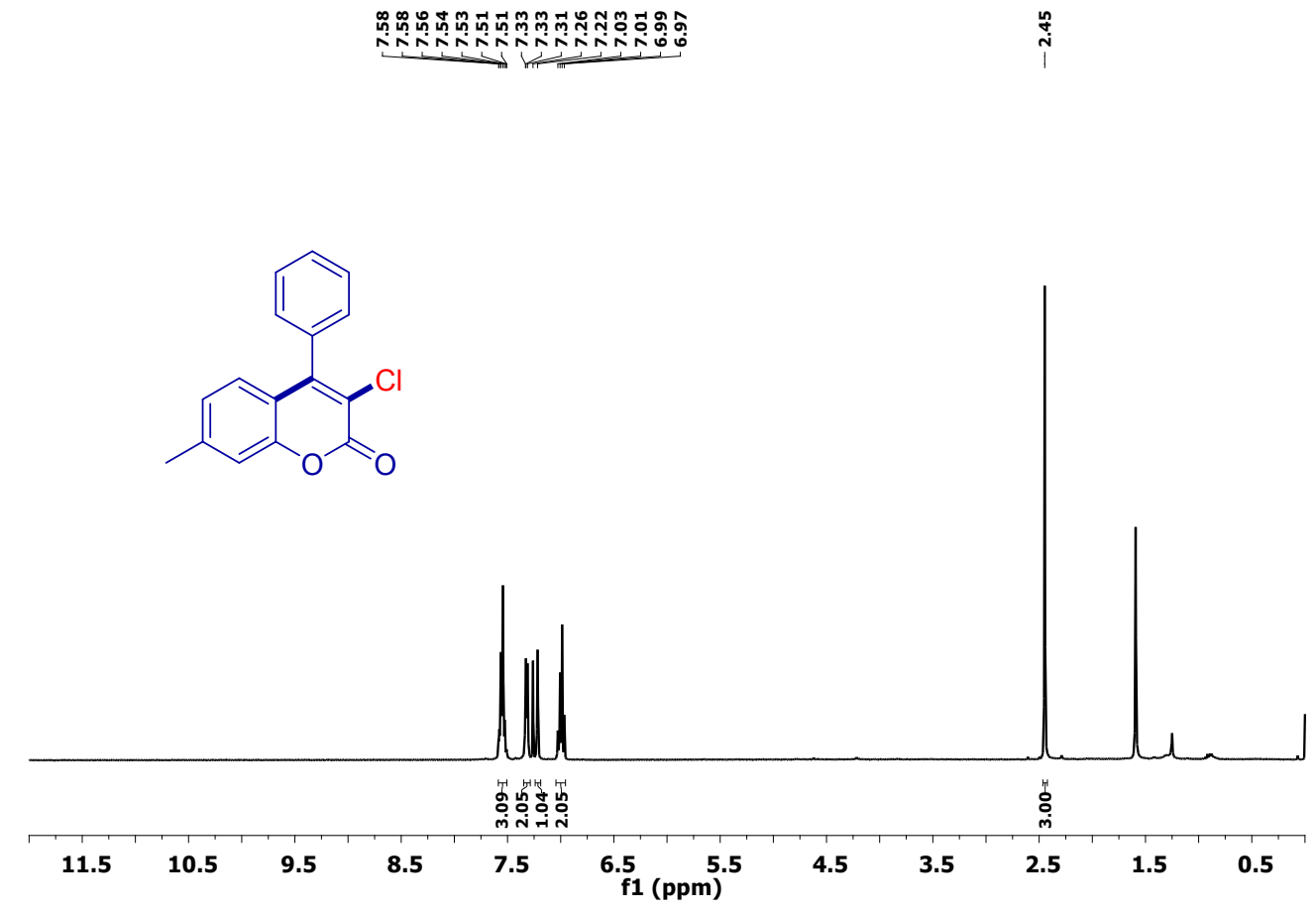

Fig. S35. ${ }^{1} \mathrm{H}$ NMR $\left(400 \mathrm{MHz}, \mathrm{CDCl}_{3}\right)$ spectrum of 3-chloro-7-methyl-4-phenyl-2H-chromen-2one (2o).

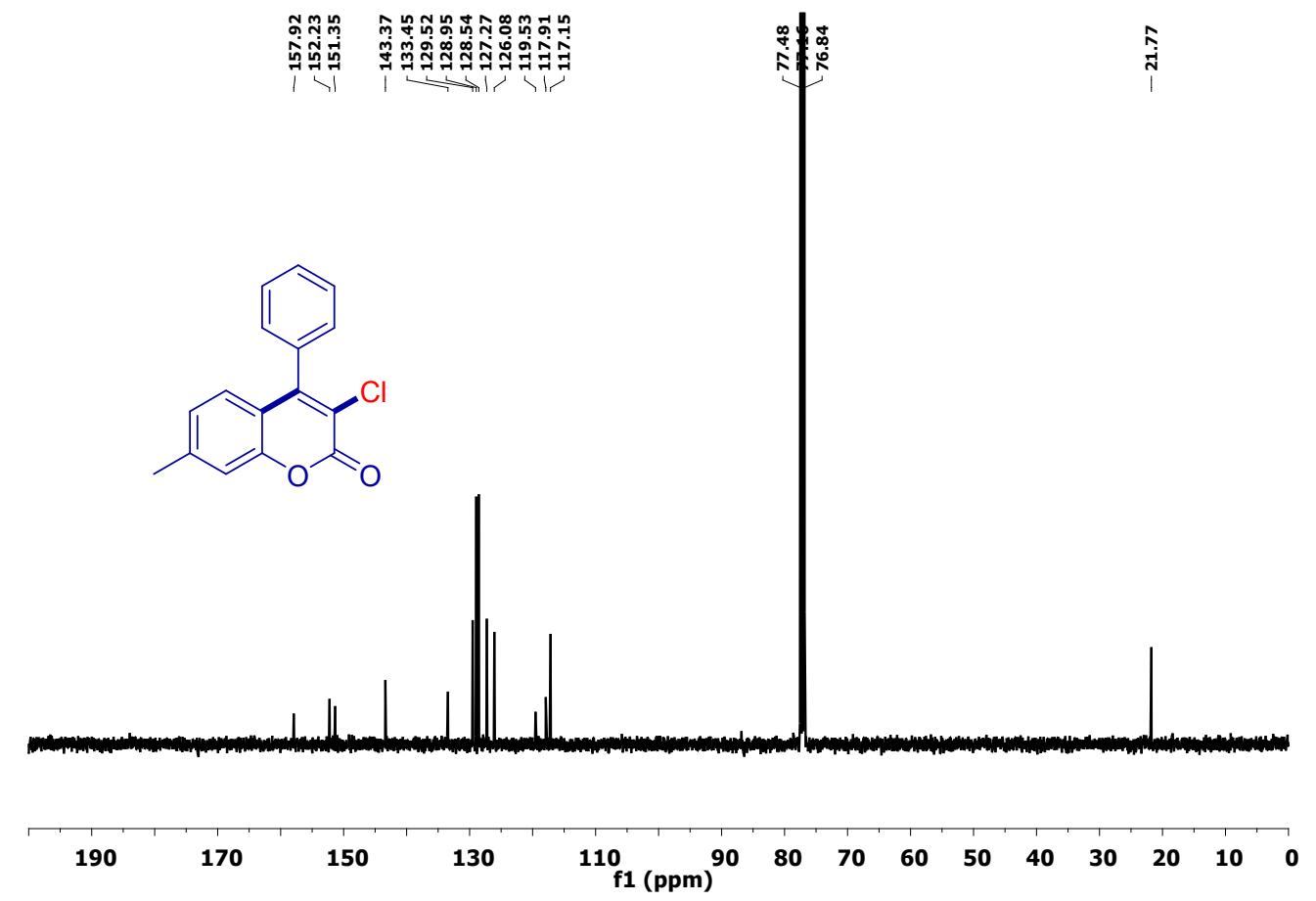

Fig. S36. ${ }^{13} \mathrm{C} \mathrm{NMR}\left(100 \mathrm{MHz}, \mathrm{CDCl}_{3}\right)$ spectrum of 3-chloro-7-methyl-4-phenyl-2H-chromen-2one (20). 

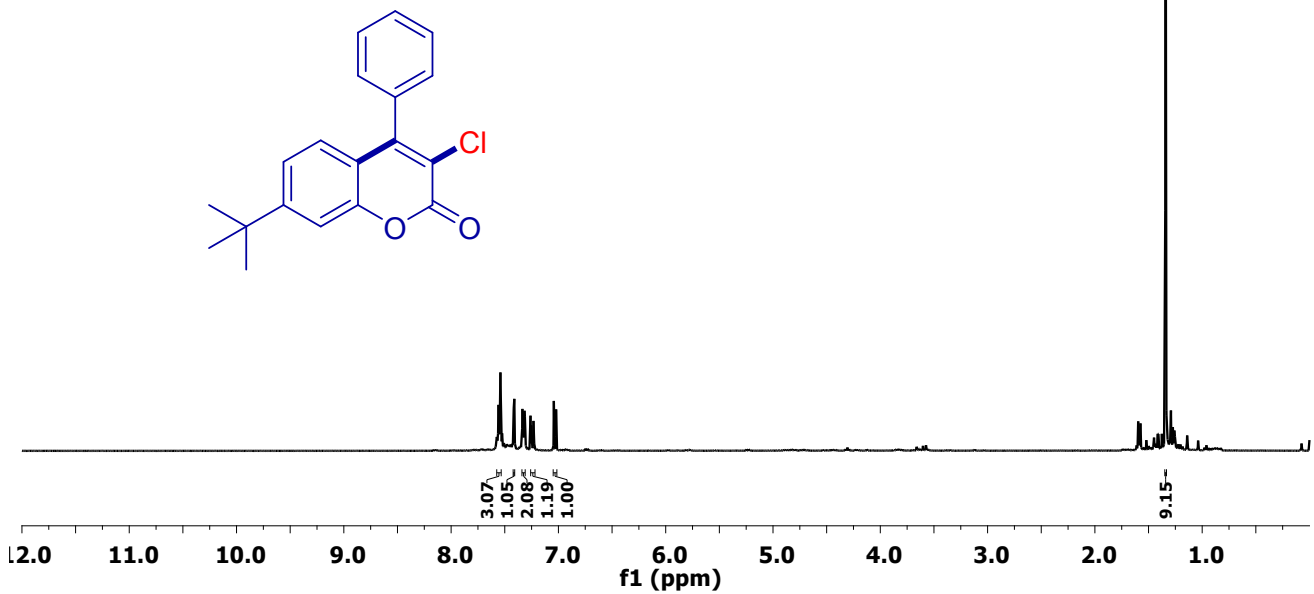

Fig. S37. ${ }^{1} \mathrm{H}$ NMR (400 MHz, $\mathrm{CDCl}_{3}$ ) spectrum of 7-(tert-butyl)-3-chloro-4-phenyl-2Hchromen-2-one (2p).

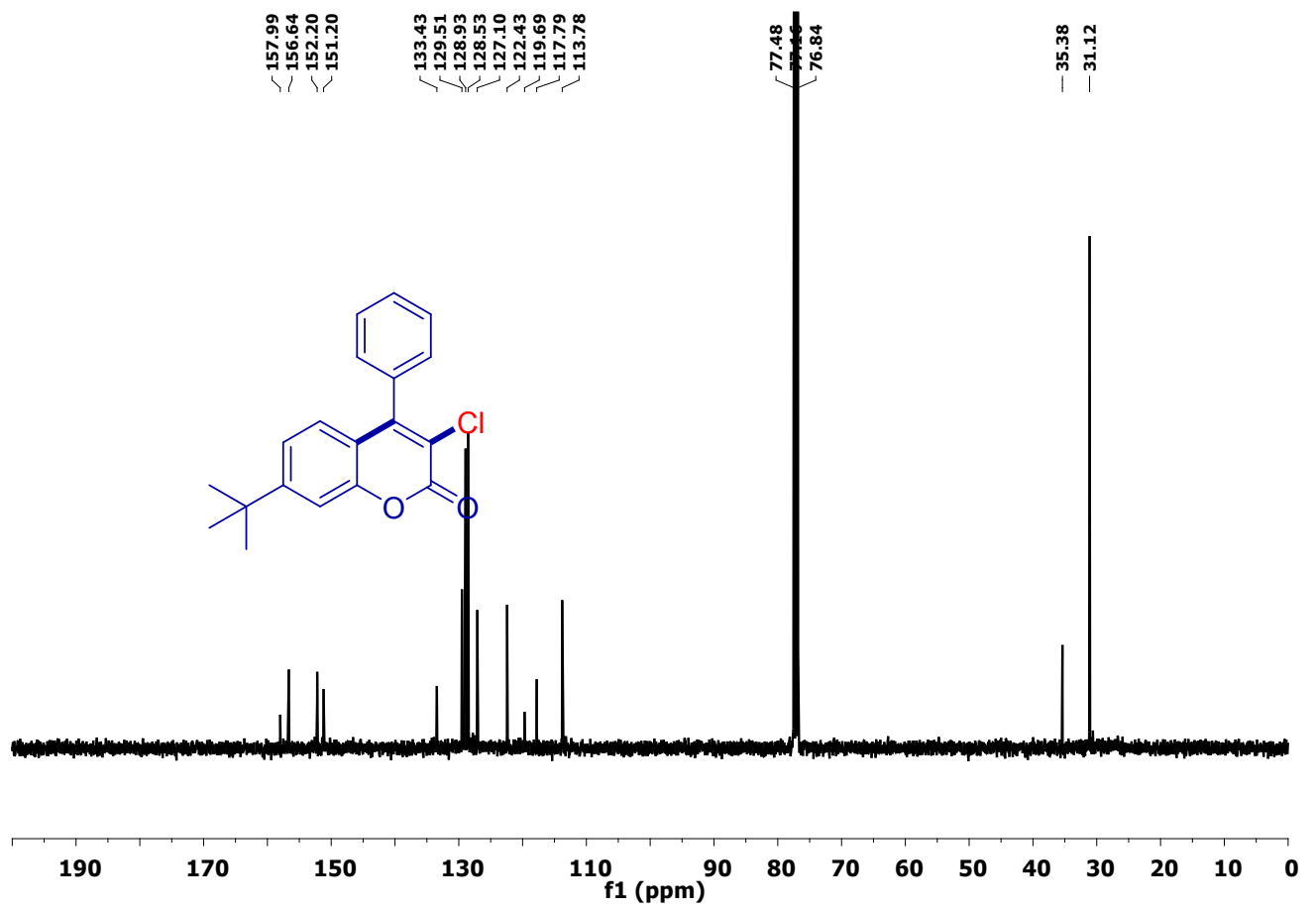

Fig. S38. ${ }^{13} \mathrm{C} \mathrm{NMR}\left(100 \mathrm{MHz}, \mathrm{CDCl}_{3}\right)$ spectrum of 7-(tert-butyl)-3-chloro-4-phenyl-2Hchromen-2-one (2p). 


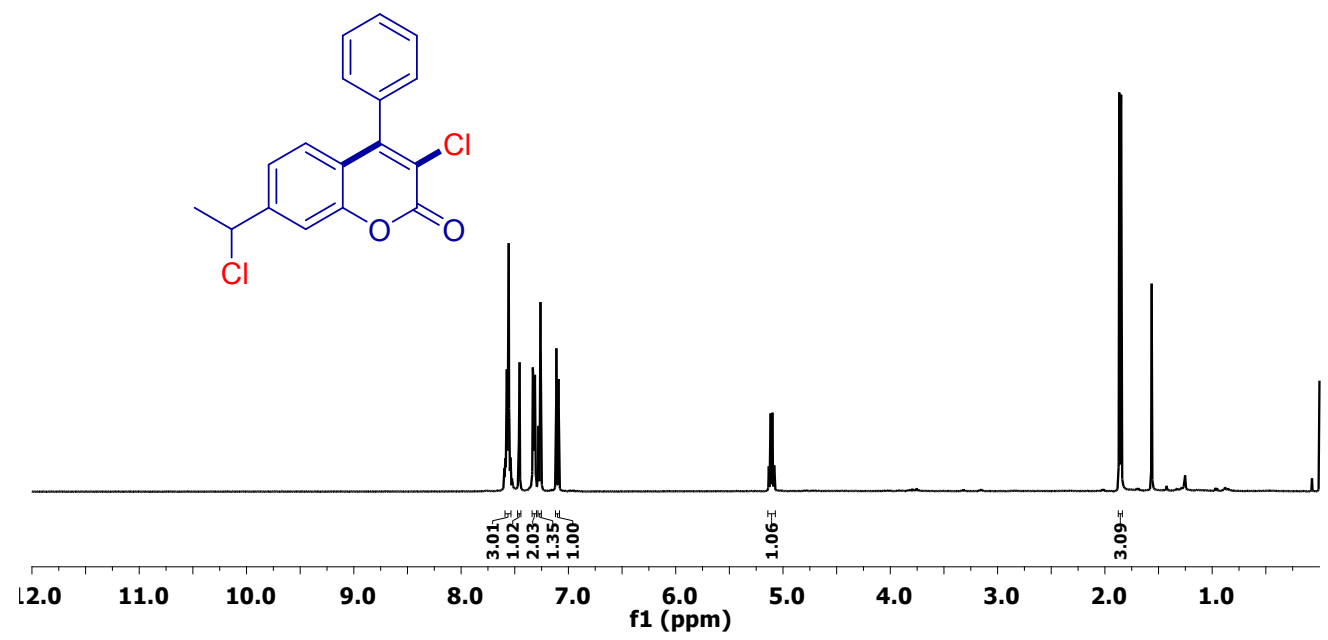

Fig. S39. ${ }^{1} \mathrm{H}$ NMR (400 MHz, $\mathrm{CDCl}_{3}$ ) spectrum of 3-chloro-7-(1-chloroethyl)-4-phenyl-2Hchromen-2-one (2q).

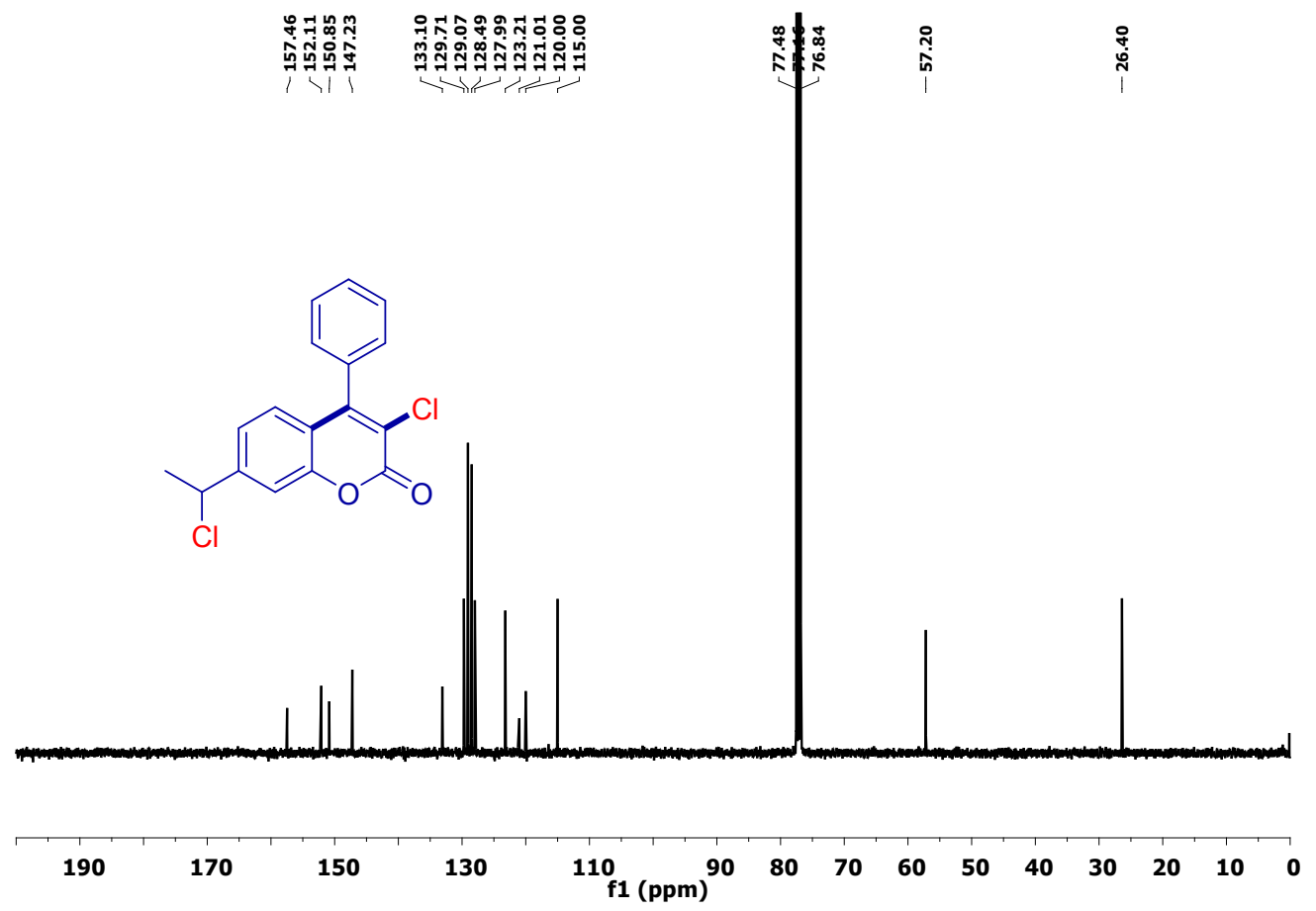

Fig. S40. ${ }^{13} \mathrm{C}$ NMR (100 MHz, $\mathrm{CDCl}_{3}$ ) spectrum of 3-chloro-7-(1-chloroethyl)-4-phenyl-2Hchromen-2-one (2q). 


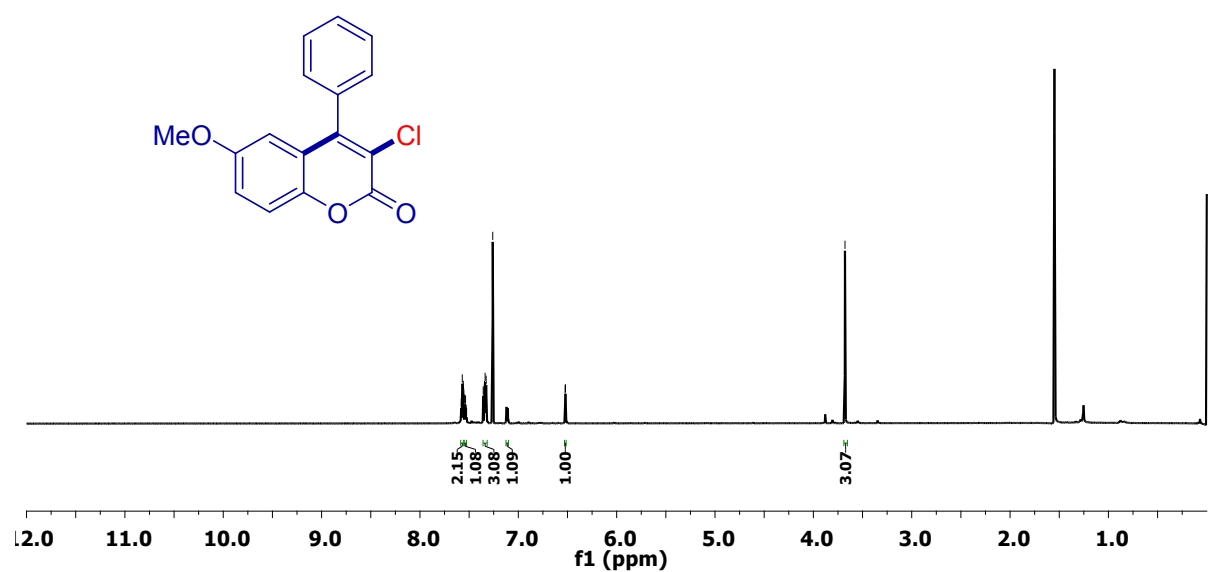

Fig. S41. ${ }^{1} \mathrm{H}$ NMR $\left(700 \mathrm{MHz}, \mathrm{CDCl}_{3}\right)$ spectrum of 3-chloro-6-methoxy-4-phenyl-2H-chromen2-one (2s).

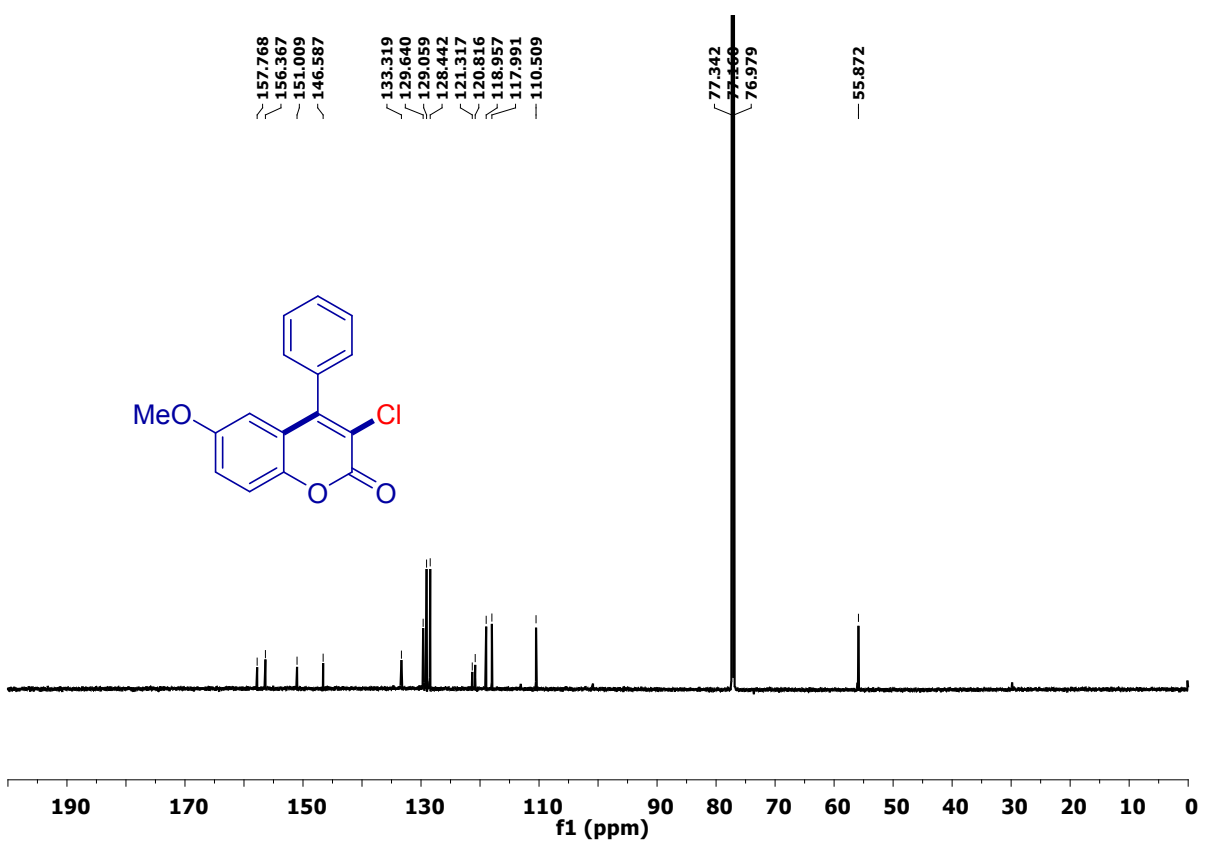

Fig. S42. ${ }^{13} \mathrm{C}$ NMR (175 MHz, $\left.\mathrm{CDCl}_{3}\right)$ spectrum of 3-chloro-6-methoxy-4-phenyl-2H-chromen2-one (2s). 


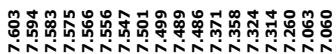
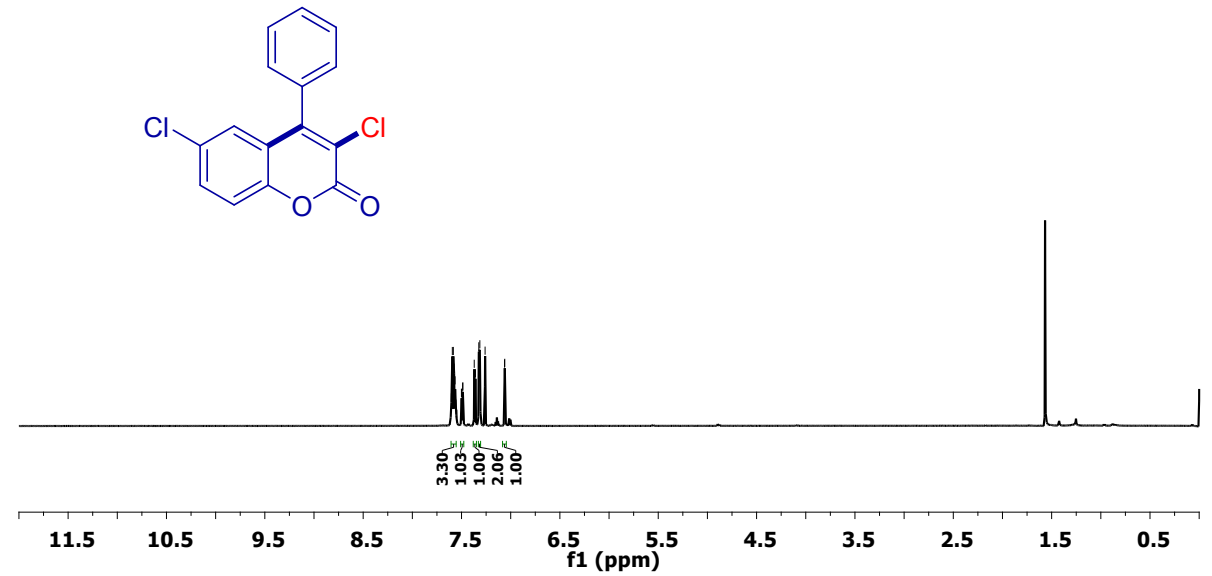

Fig. S43. ${ }^{1} \mathrm{H}$ NMR (700 MHz, $\left.\mathrm{CDCl}_{3}\right)$ spectrum of 3,6-dichloro-4-phenyl-2H-chromen-2-one (2t).

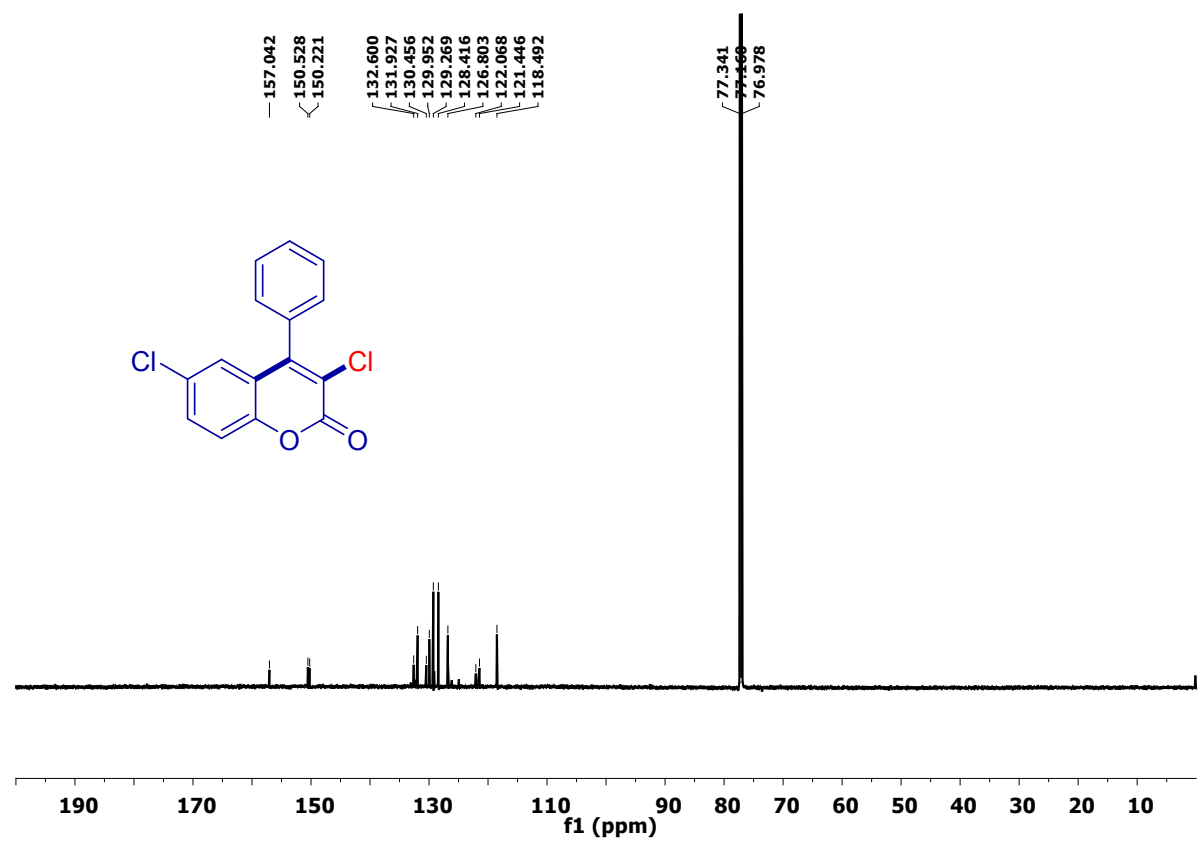

Fig. S44. ${ }^{13} \mathrm{C}$ NMR $\left(175 \mathrm{MHz}, \mathrm{CDCl}_{3}\right)$ spectrum of 3,6-dichloro-4-phenyl-2H-chromen-2-one (2t). 


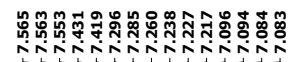
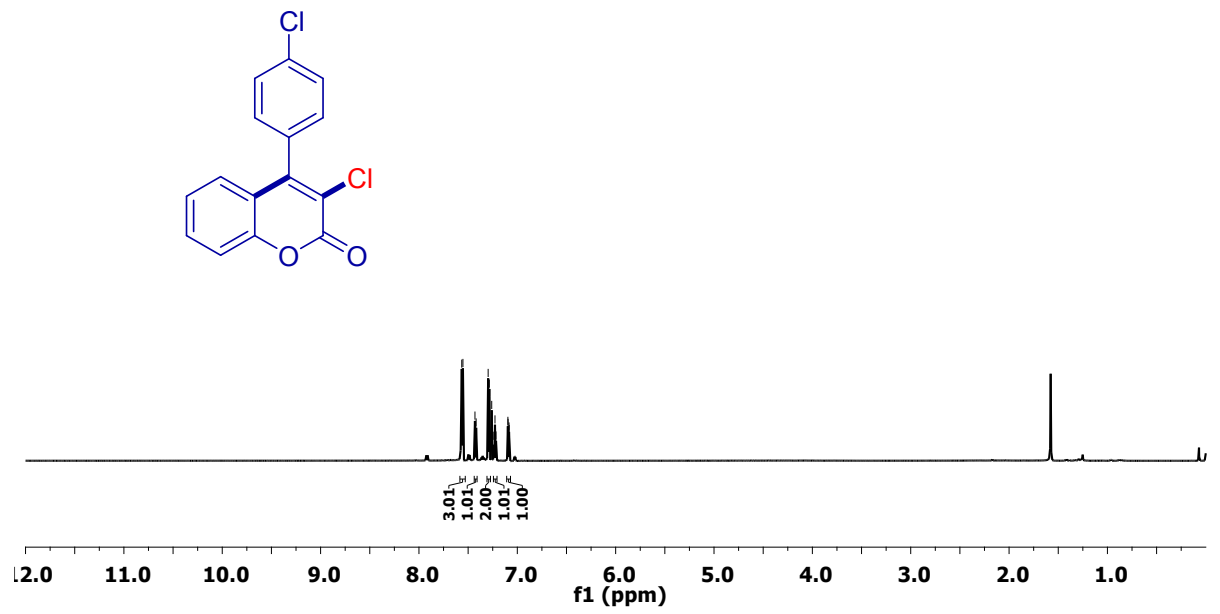

Fig. S45. ${ }^{1} \mathrm{H}$ NMR (700 MHz, $\mathrm{CDCl}_{3}$ ) spectrum of 3-chloro-4-(4-chlorophenyl)-2H-chromen-2one (4a).

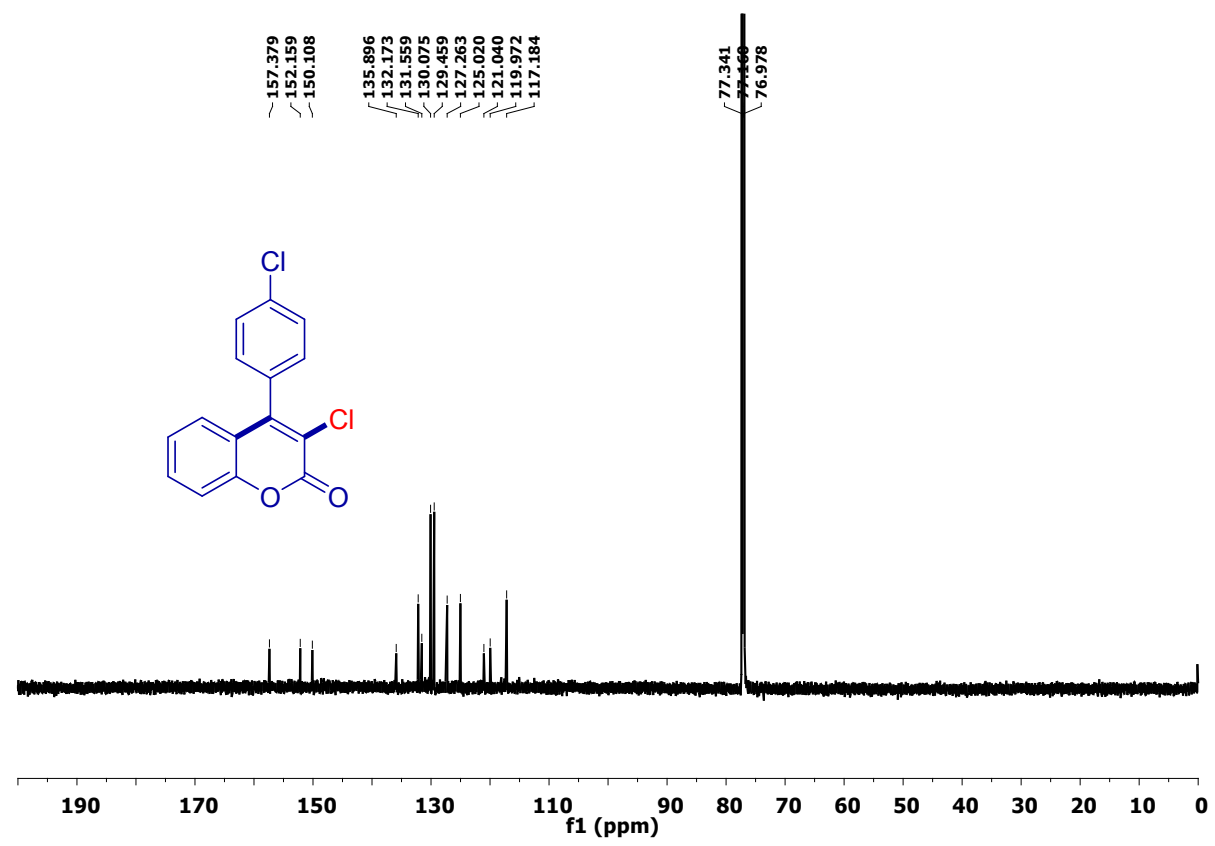

Fig. S46. ${ }^{13} \mathrm{C} \mathrm{NMR}\left(175 \mathrm{MHz}, \mathrm{CDCl}_{3}\right)$ spectrum of 3-chloro-4-(4-chlorophenyl)-2H-chromen-2one (4a). 


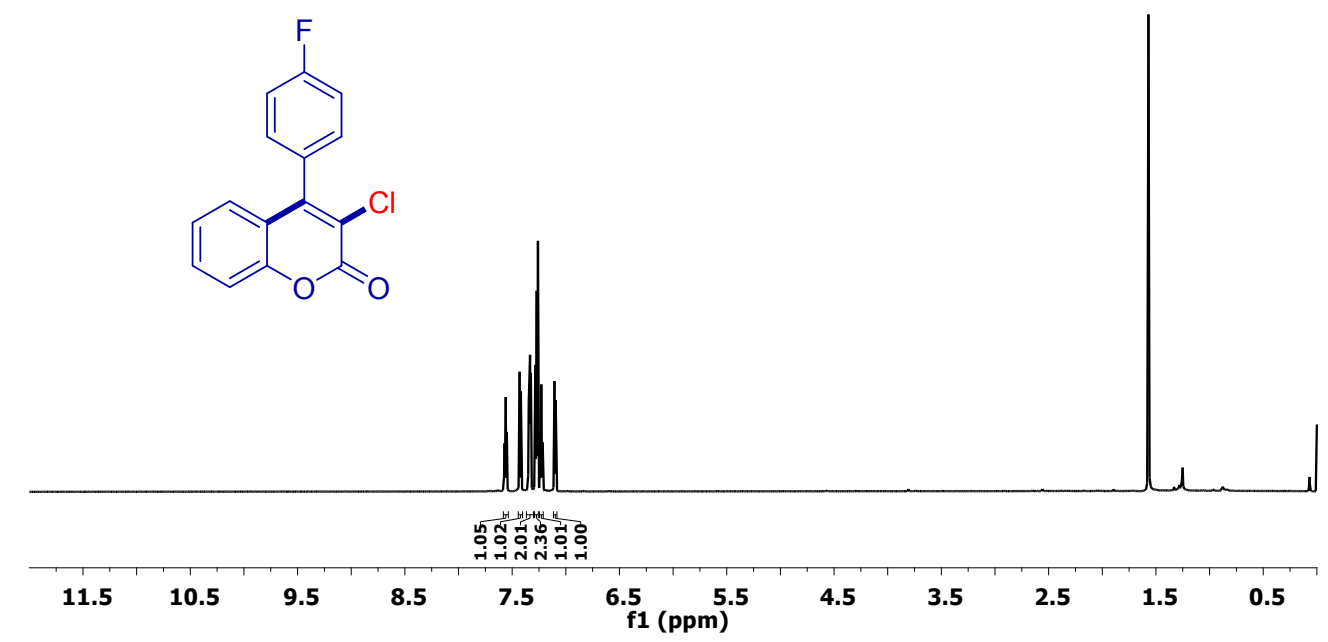

Fig. S47. ${ }^{1} \mathrm{H}$ NMR (700 MHz, $\mathrm{CDCl}_{3}$ ) spectrum of 3-chloro-4-(4-fluorophenyl)-2H-chromen-2one (4b).
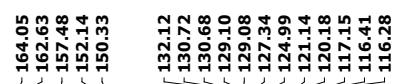<smiles>O=c1oc2ccccc2c(-c2ccc(F)cc2)c1Cl</smiles>
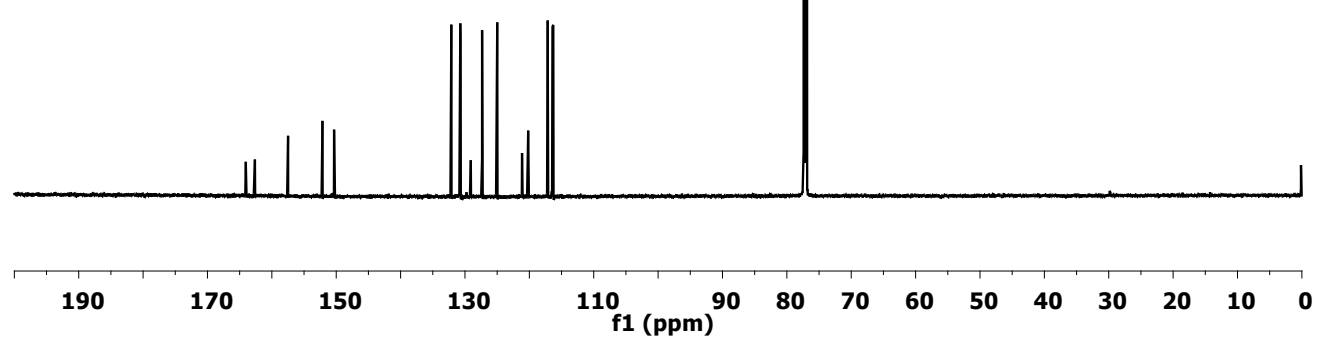

Fig. S48. ${ }^{13} \mathrm{C}$ NMR (175 MHz, $\mathrm{CDCl}_{3}$ ) spectrum of 3-chloro-4-(4-fluorophenyl)-2H-chromen-2one (4b). 


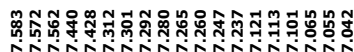 \\ (}

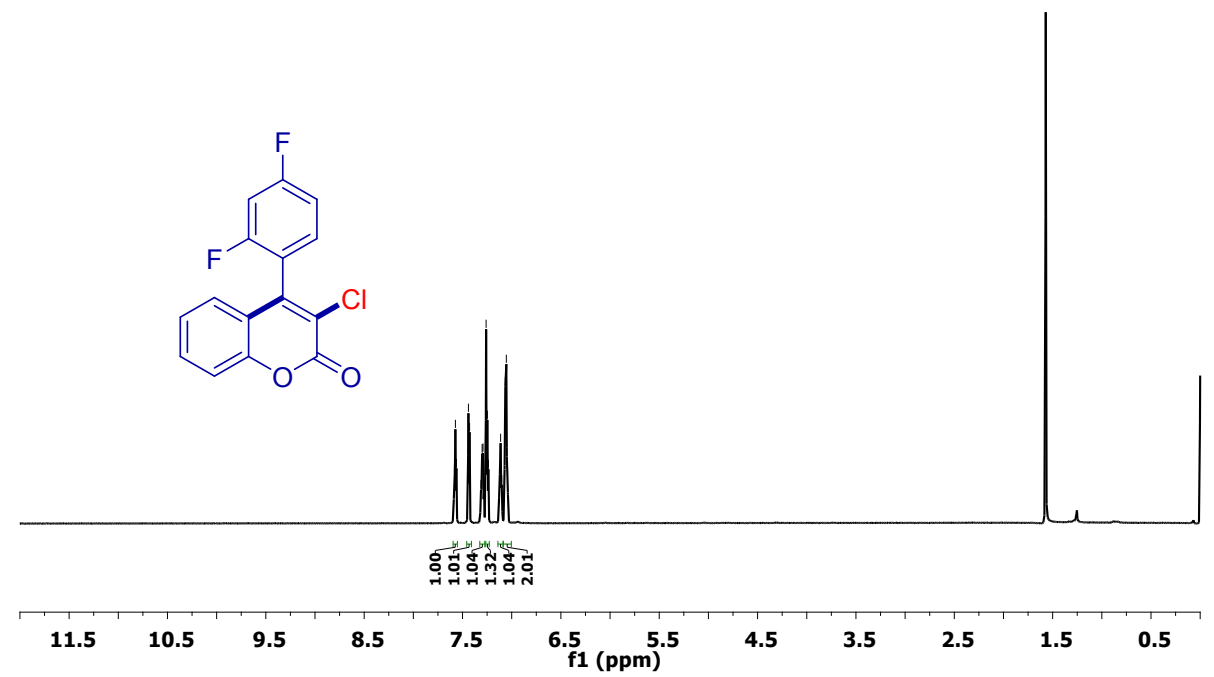

Fig. S49. ${ }^{1} \mathrm{H}$ NMR (700 MHz, $\mathrm{CDCl}_{3}$ ) spectrum of 3-chloro-4-(2,4-difluorophenyl)-2Hchromen-2-one (4c).

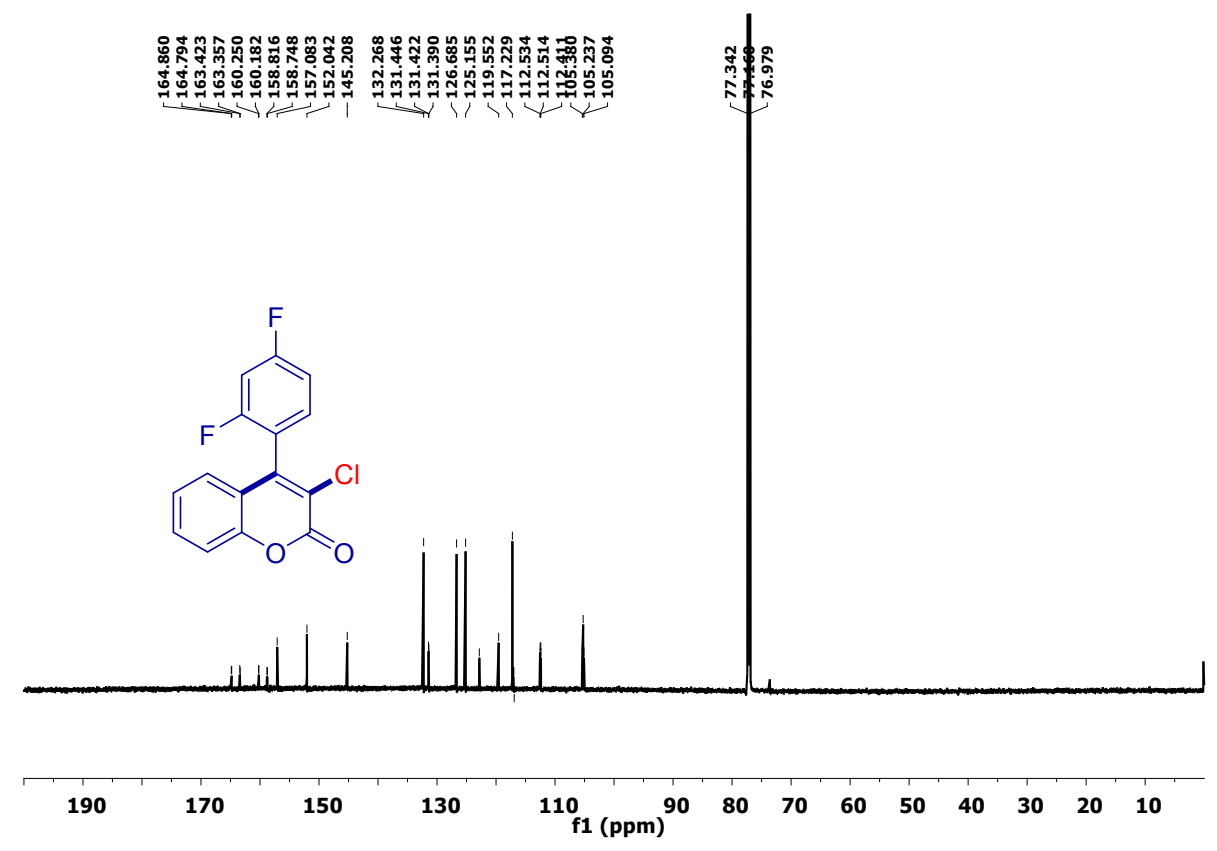

Fig. S50. ${ }^{13} \mathrm{C}$ NMR (175 MHz, $\mathrm{CDCl}_{3}$ ) spectrum of 3-chloro-4-(2,4-difluorophenyl)-2Hchromen-2-one (4c). 


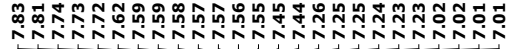

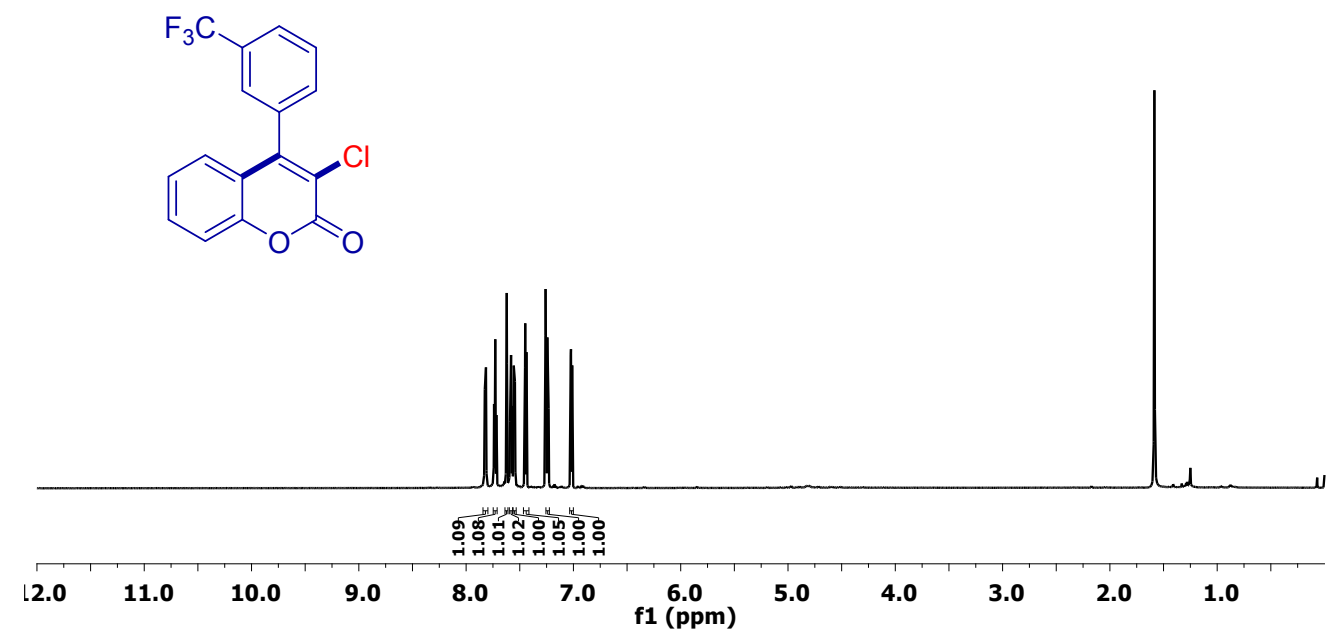

Fig. S51. ${ }^{1} \mathrm{H} \mathrm{NMR}\left(700 \mathrm{MHz}, \mathrm{CDCl}_{3}\right)$ spectrum of 3-chloro-4-(3-(trifluoromethyl)phenyl)-2Hchromen-2-one (4d).

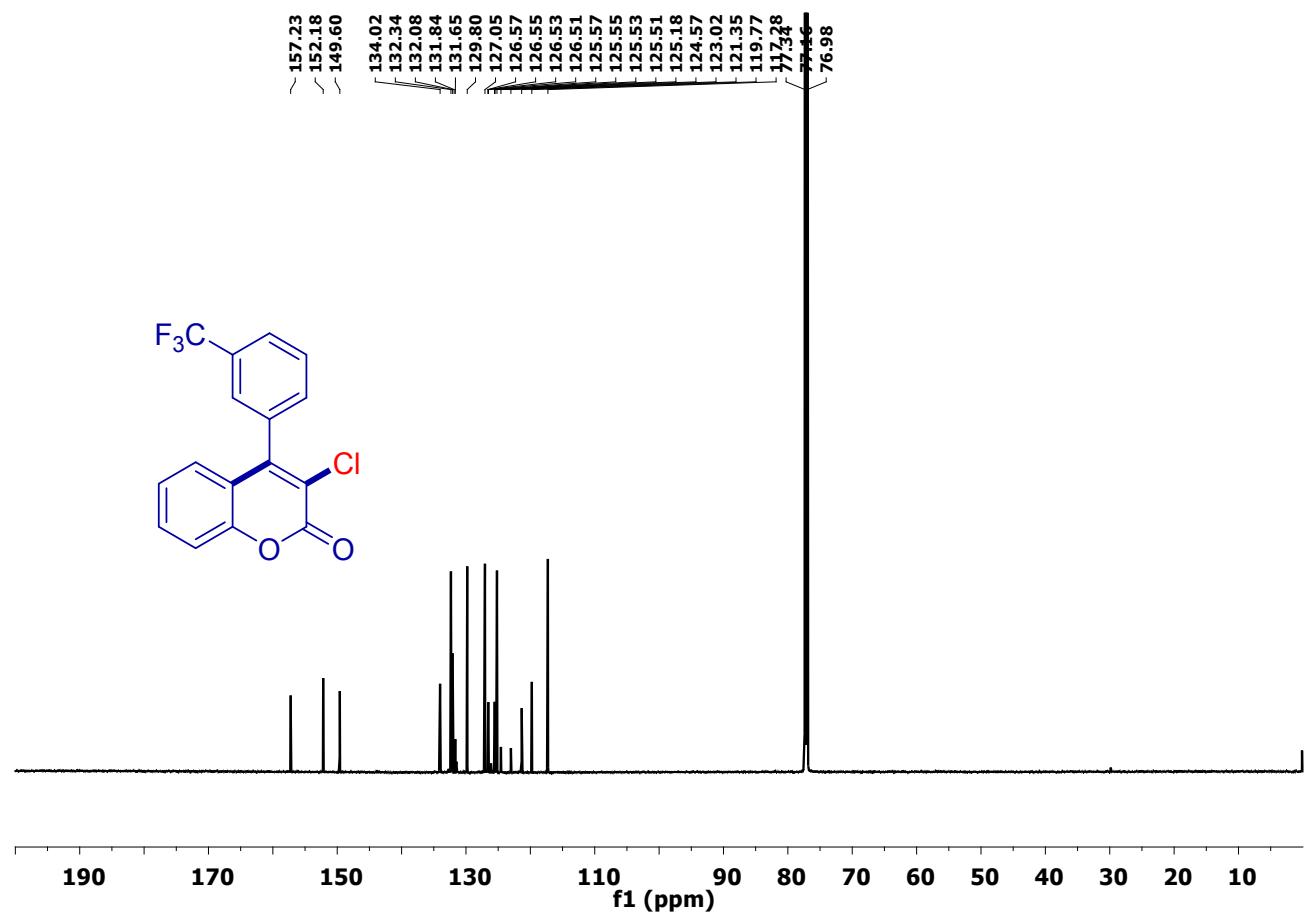

Fig. S52. ${ }^{13} \mathrm{C} \mathrm{NMR}\left(175 \mathrm{MHz}, \mathrm{CDCl}_{3}\right)$ spectrum of 3-chloro-4-(3-(trifluoromethyl)phenyl)-2Hchromen-2-one (4d). 


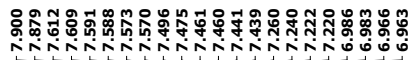
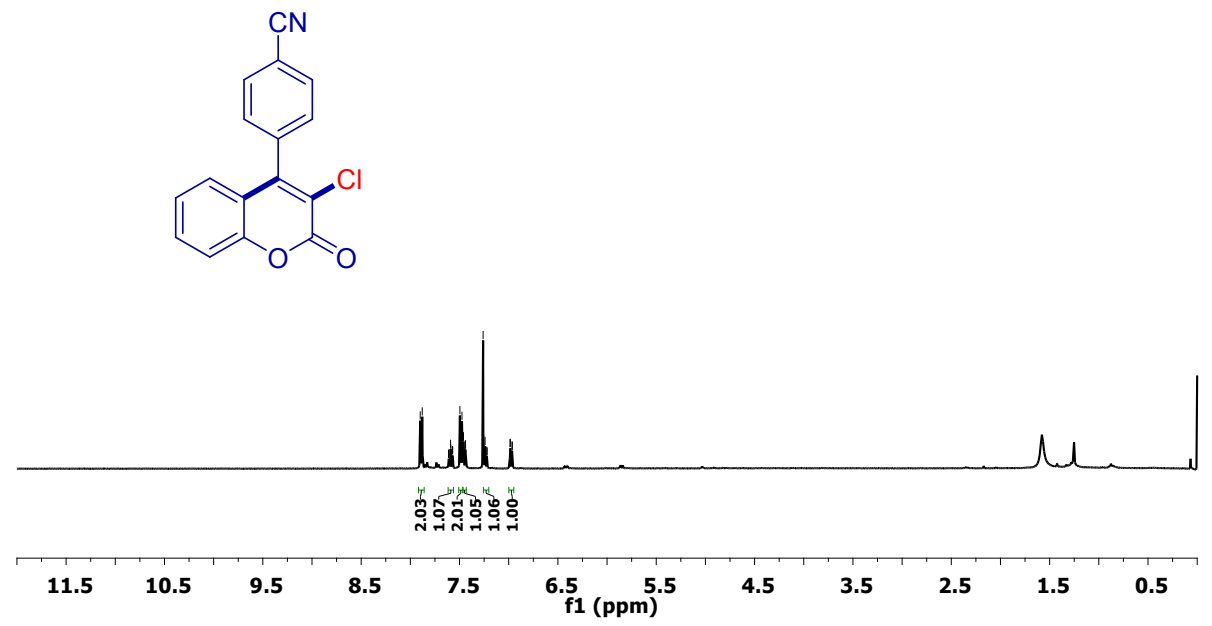

Fig. S53. ${ }^{1} \mathrm{H}$ NMR (400 MHz, $\left.\mathrm{CDCl}_{3}\right)$ spectrum of 4-(3-chloro-2-oxo-2H-chromen-4yl)benzonitrile (4e).

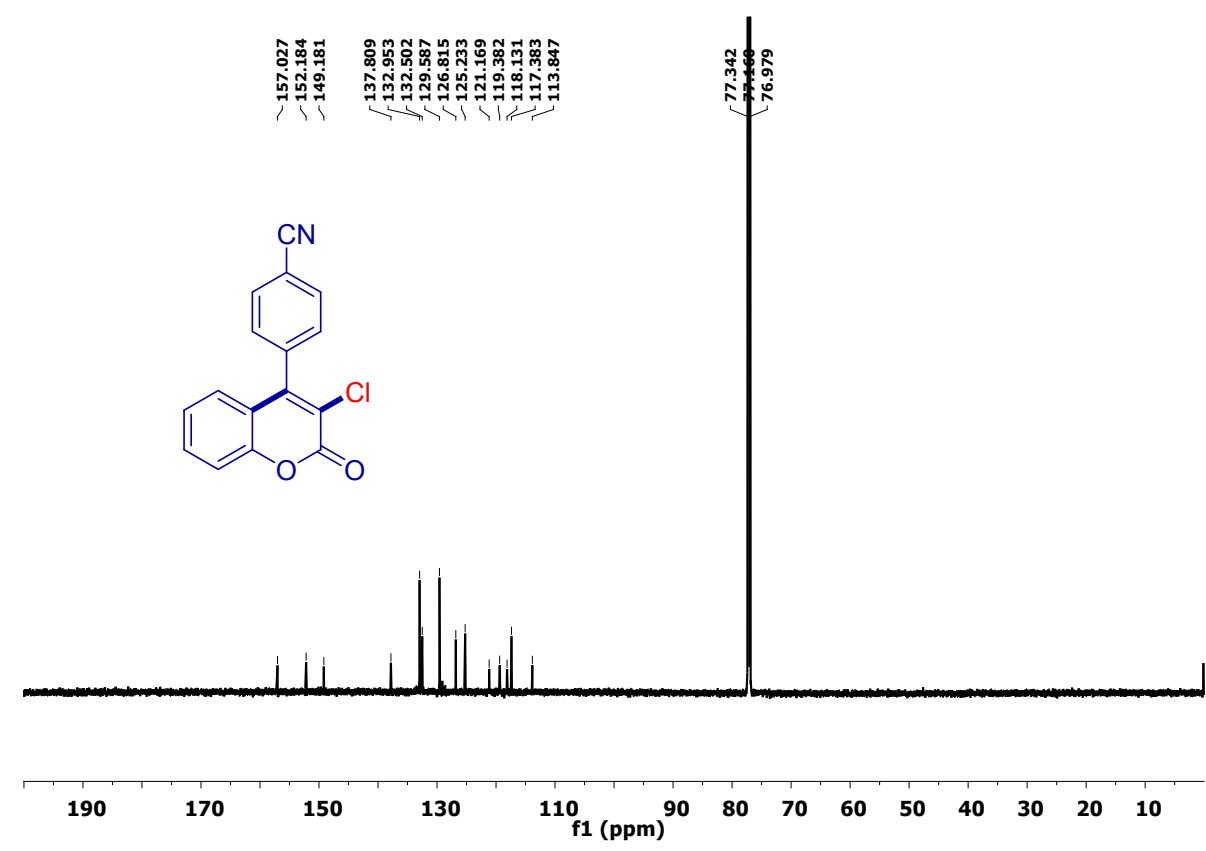

Fig. S54. ${ }^{13} \mathrm{C}$ NMR (175 MHz, $\mathrm{CDCl}_{3}$ ) spectrum of 4-(3-chloro-2-oxo-2H-chromen-4yl)benzonitrile (4e). 


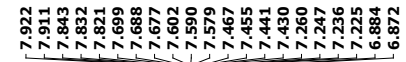
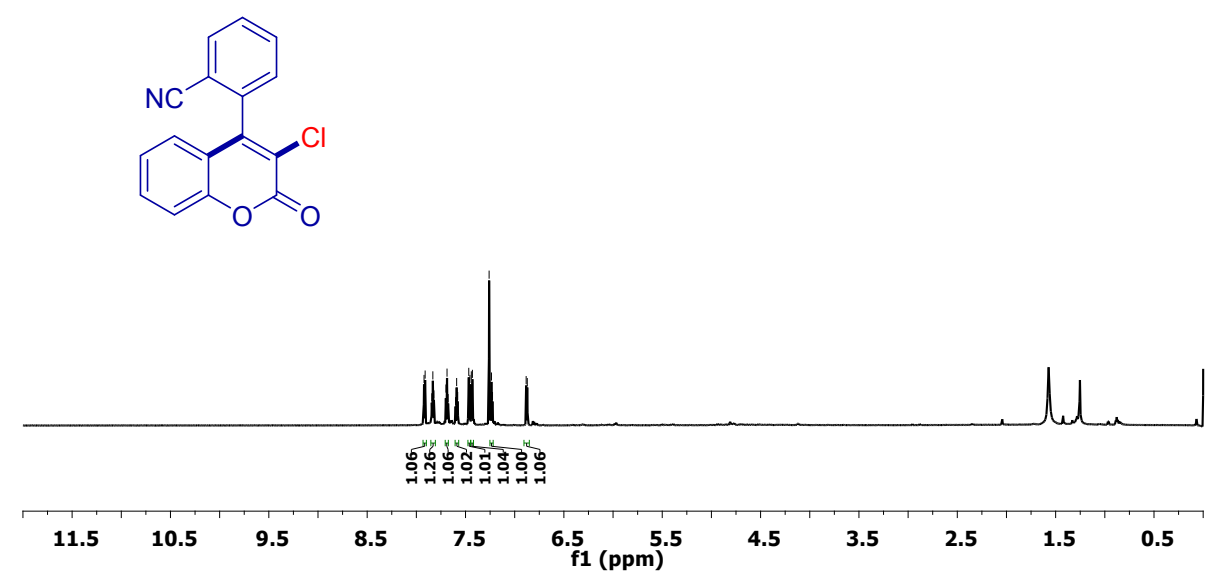

Fig. S55. ${ }^{1} \mathrm{H} \mathrm{NMR}\left(700 \mathrm{MHz}, \mathrm{CDCl}_{3}\right)$ spectrum of 2-(3-chloro-2-oxo-2H-chromen-4yl)benzonitrile (4f).

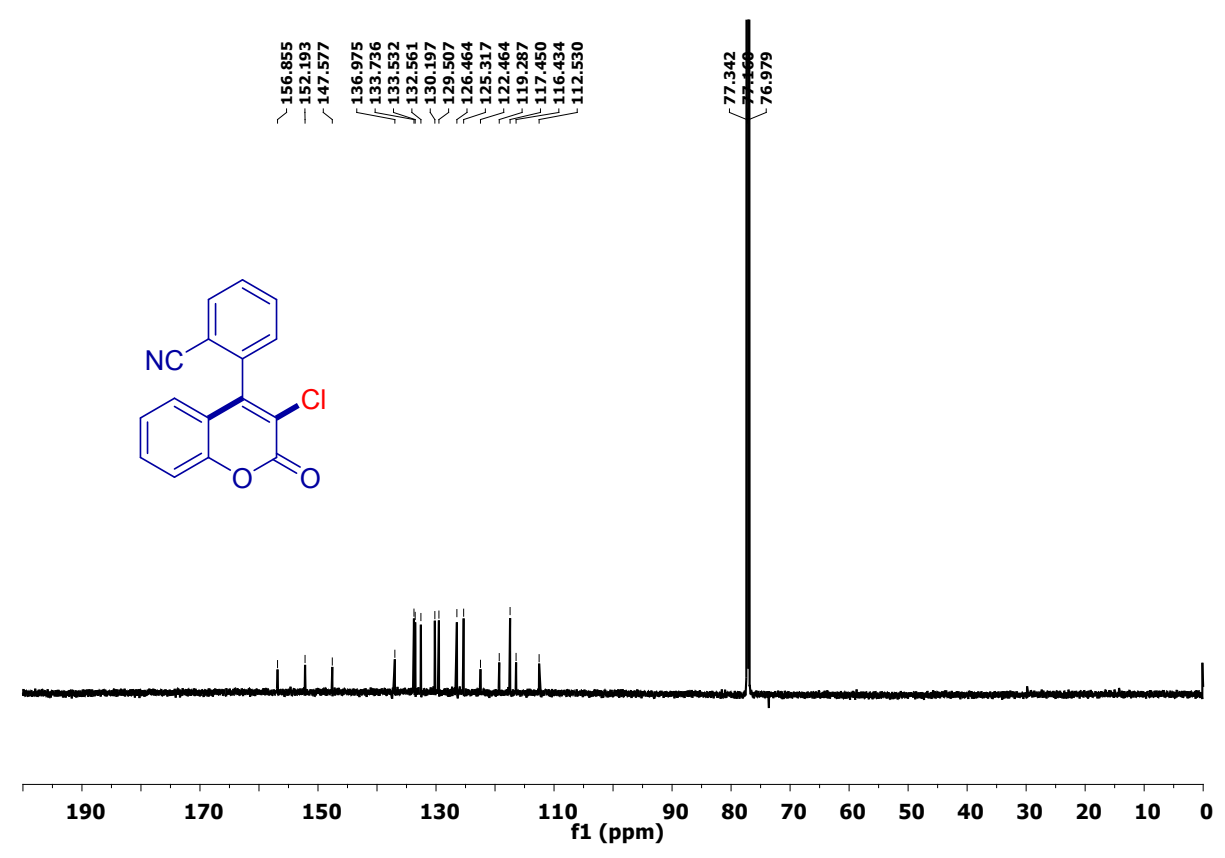

Fig. S56. ${ }^{13} \mathrm{C}$ NMR $\left(175 \mathrm{MHz}, \mathrm{CDCl}_{3}\right)$ spectrum of 2-(3-chloro-2-oxo-2H-chromen-4yl)benzonitrile (4f). 

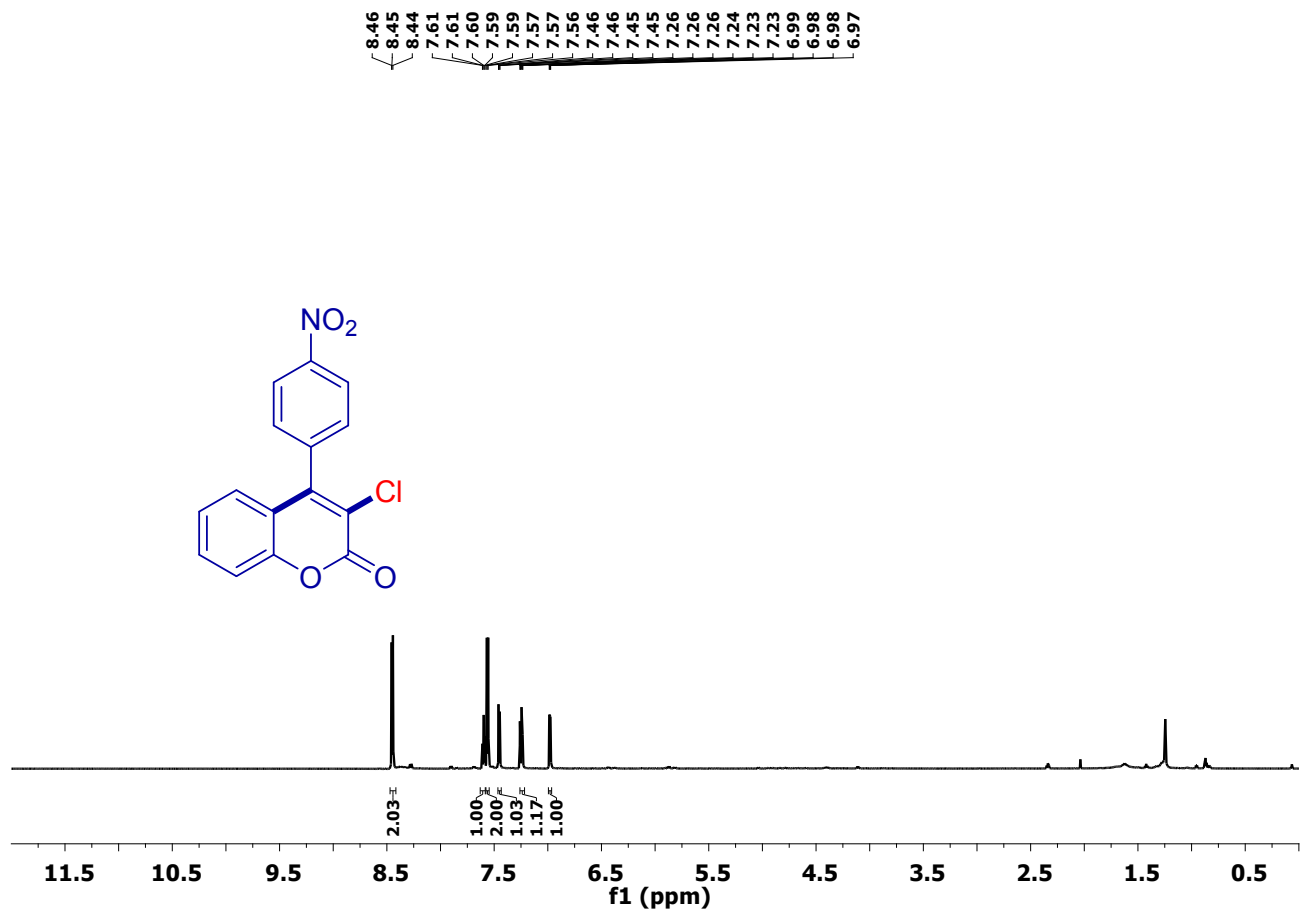

Fig. S57. ${ }^{1} \mathrm{H}$ NMR (700 MHz, $\mathrm{CDCl}_{3}$ ) spectrum of 3-chloro-4-(4-nitrophenyl)-2H-chromen-2one (4g).

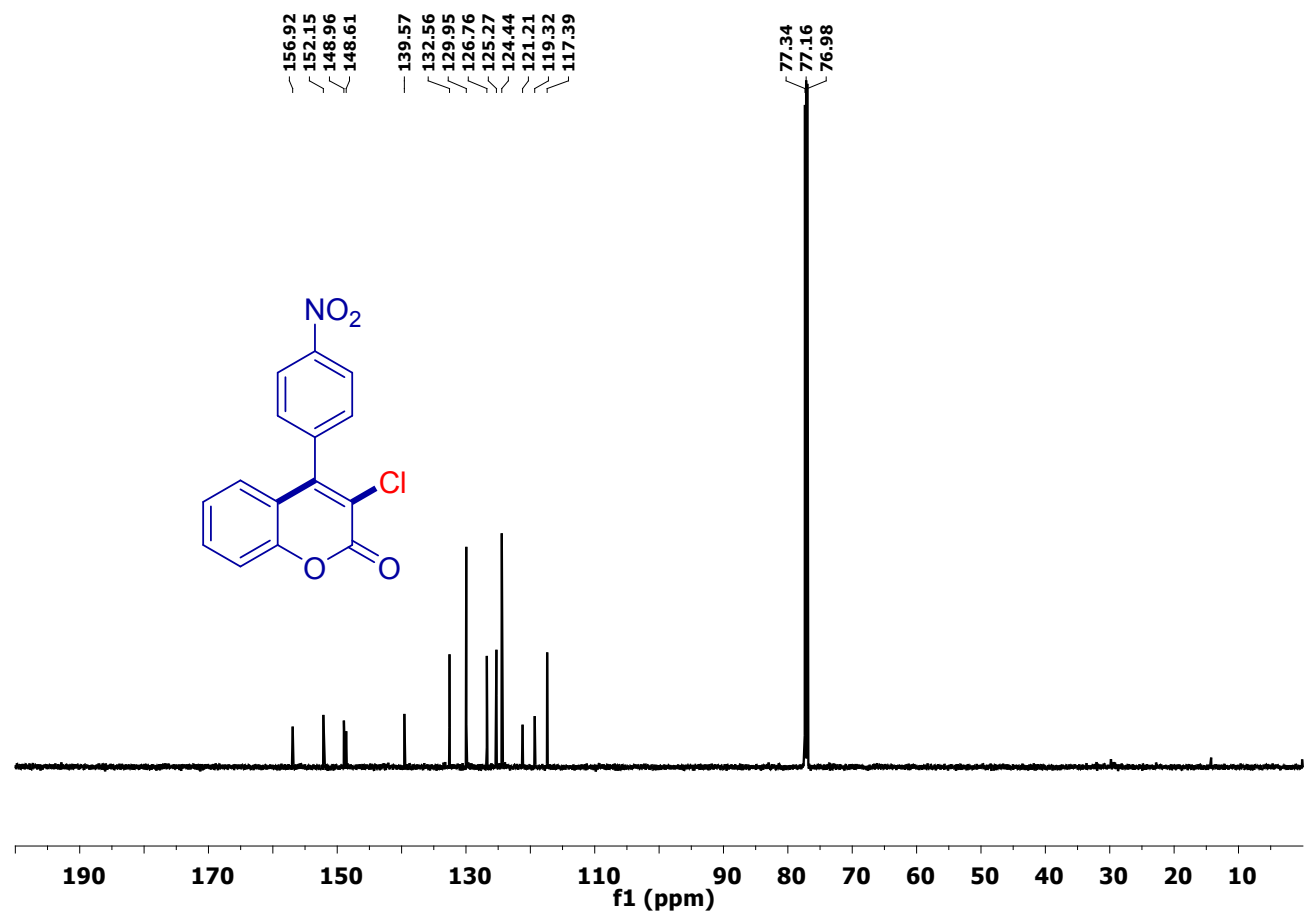

Fig. S58. ${ }^{13} \mathrm{C} \mathrm{NMR}\left(175 \mathrm{MHz}, \mathrm{CDCl}_{3}\right)$ spectrum of 3-chloro-4-(4-nitrophenyl)-2H-chromen-2one (4g). 

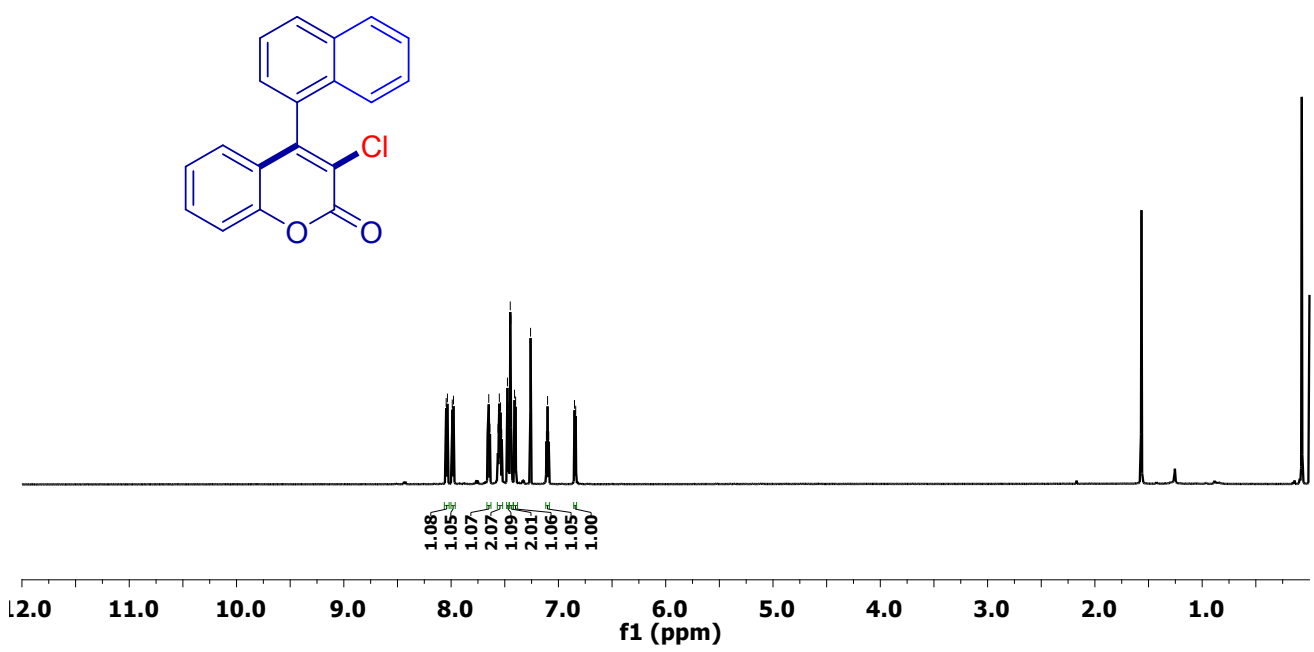

Fig. S59. ${ }^{1} \mathrm{H} \mathrm{NMR}\left(700 \mathrm{MHz}, \mathrm{CDCl}_{3}\right)$ spectrum of 3-chloro-4-(naphthalen-1-yl)-2H-chromen-2one (4h).

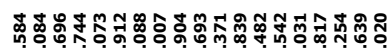
कि<smiles>O=c1oc2ccccc2c(-c2cccc3ccccc23)c1Cl</smiles>

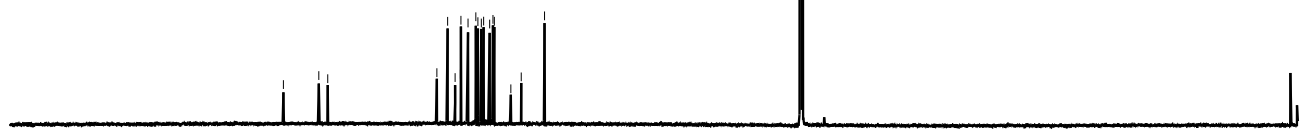

$\begin{array}{lllllllllllllll}190 & 170 & 150 & 130 & 110 & 90 & 80 & 70 & 60 & 50 & 40 & 30 & 20 & 10 & 0\end{array}$

Fig. S60. ${ }^{13} \mathrm{C}$ NMR $\left(175 \mathrm{MHz}, \mathrm{CDCl}_{3}\right)$ spectrum of 3-chloro-4-(naphthalen-1-yl)-2H-chromen2-one (4h). 


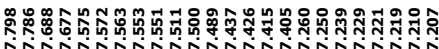
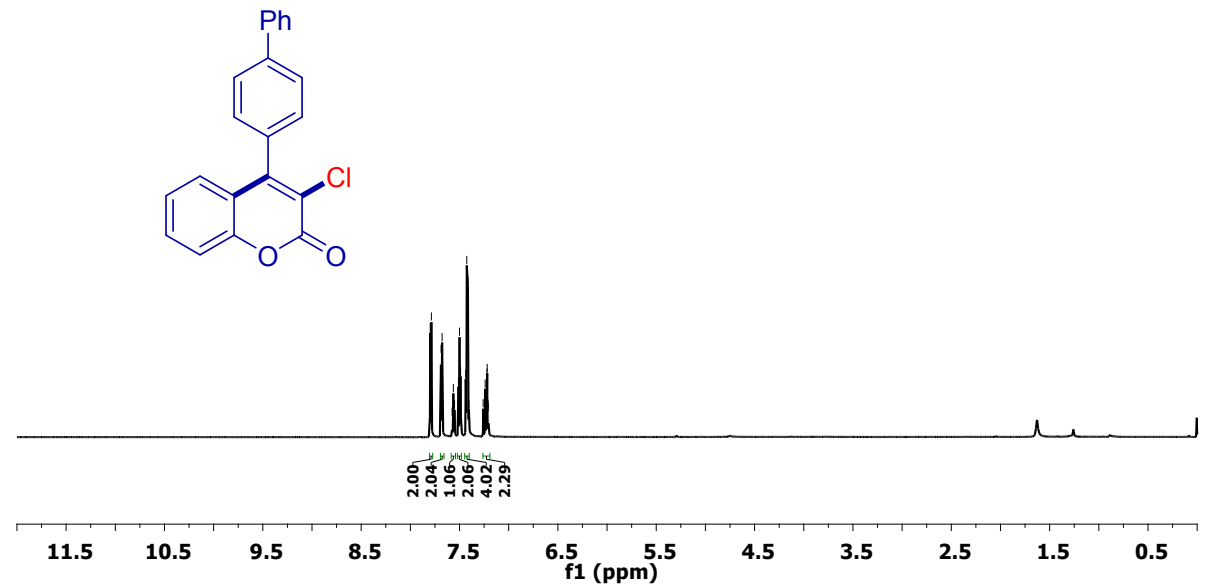

Fig. S61. ${ }^{1} \mathrm{H}$ NMR $\left(700 \mathrm{MHz}, \mathrm{CDCl}_{3}\right)$ spectrum of 4-([1,1'-biphenyl]-4-yl)-3-chloro-2Hchromen-2-one (4i).

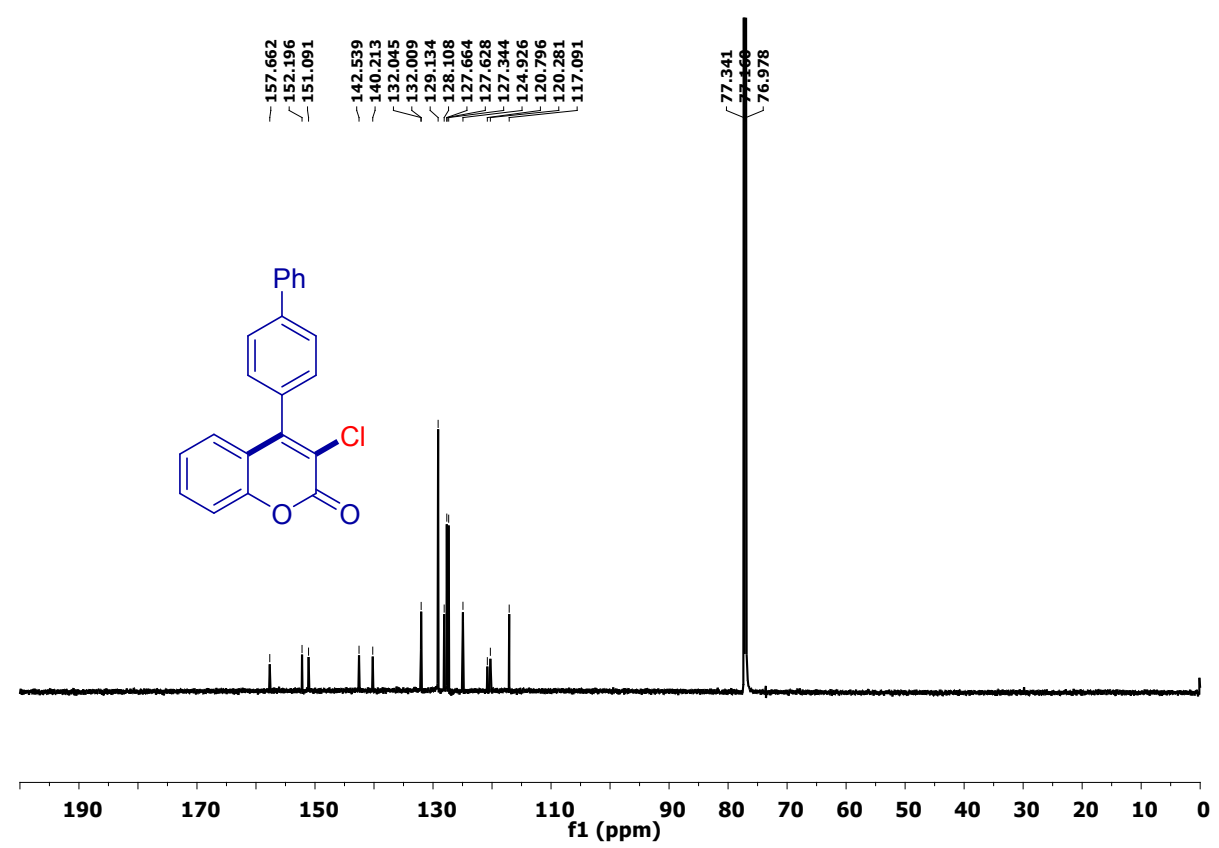

Fig. S62. ${ }^{13} \mathrm{C}$ NMR $\left(175 \mathrm{MHz}, \mathrm{CDCl}_{3}\right)$ spectrum of 4-([1,1'-biphenyl]-4-yl)-3-chloro-2Hchromen-2-one (4i). 


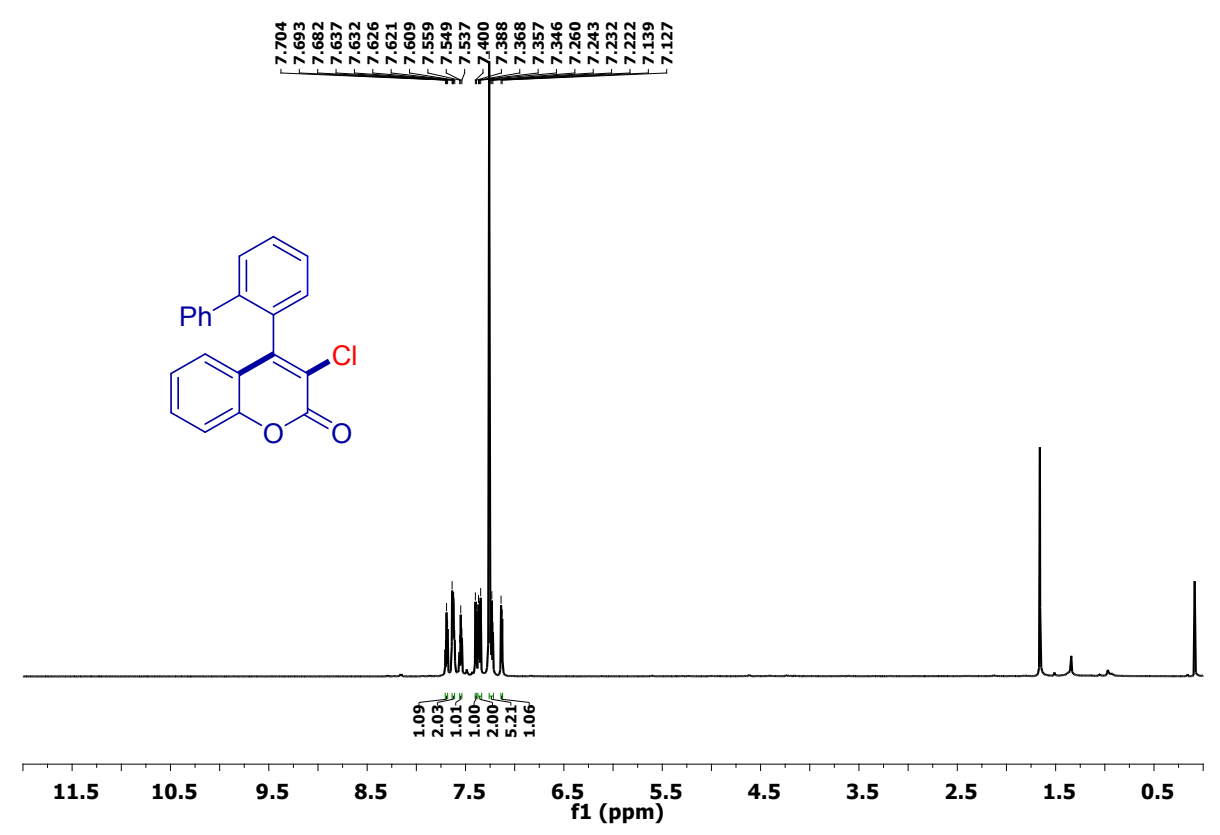

Fig. S63. ${ }^{1} \mathrm{H}$ NMR $\left(700 \mathrm{MHz}, \mathrm{CDCl}_{3}\right)$ spectrum of 4-([1,1'-biphenyl]-2-yl)-3-chloro-2Hchromen-2-one $(\mathbf{4 j})$.
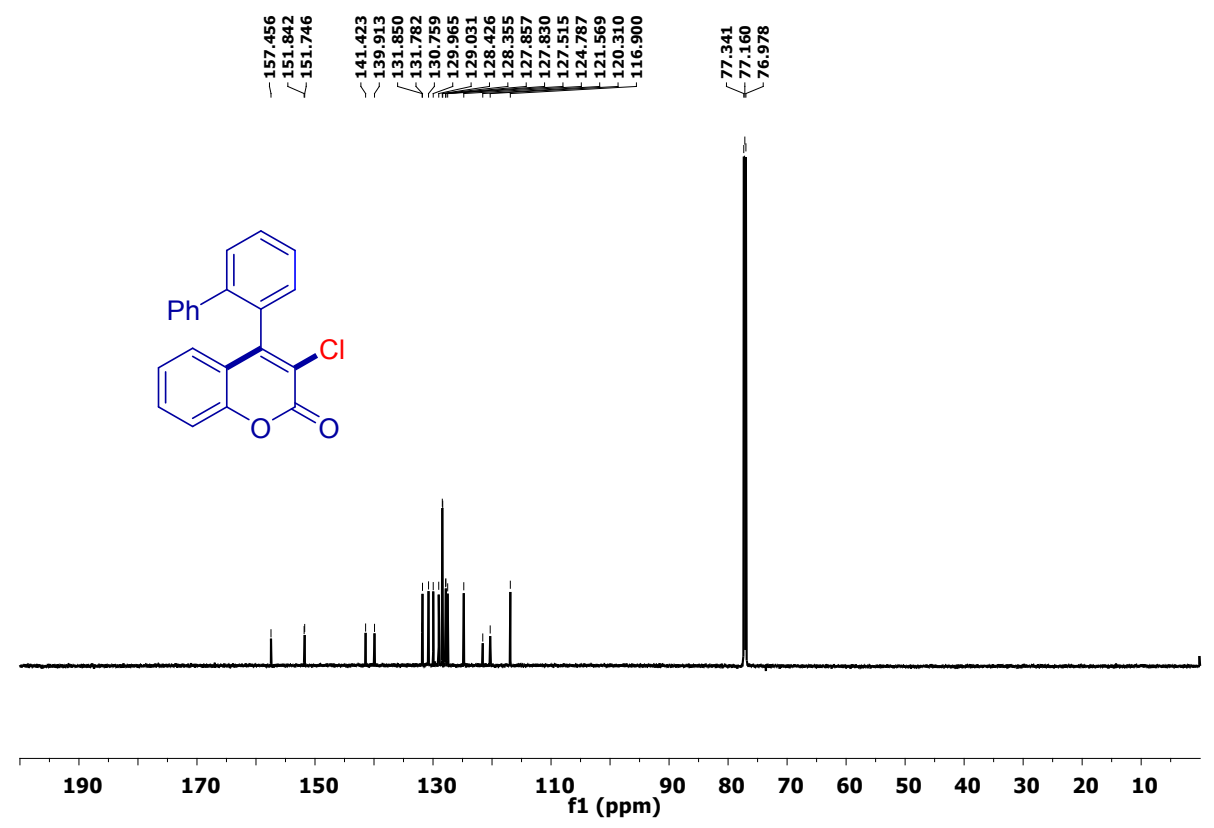

Fig. S64. ${ }^{13} \mathrm{C}$ NMR $\left(175 \mathrm{MHz}, \mathrm{CDCl}_{3}\right)$ spectrum of 4-([1,1'-biphenyl]-2-yl)-3-chloro-2H-

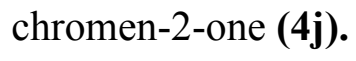




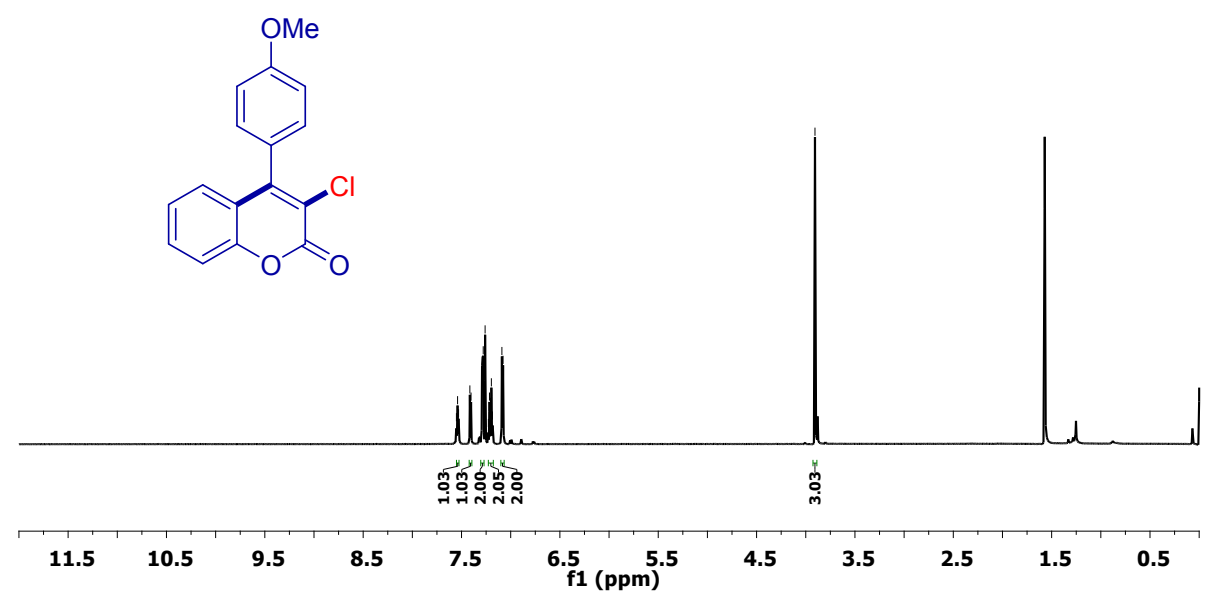

Fig. S65. ${ }^{1} \mathrm{H}$ NMR (700 MHz, $\mathrm{CDCl}_{3}$ ) spectrum of 3-chloro-4-(4-methoxyphenyl)-2H-chromen2-one (4k).

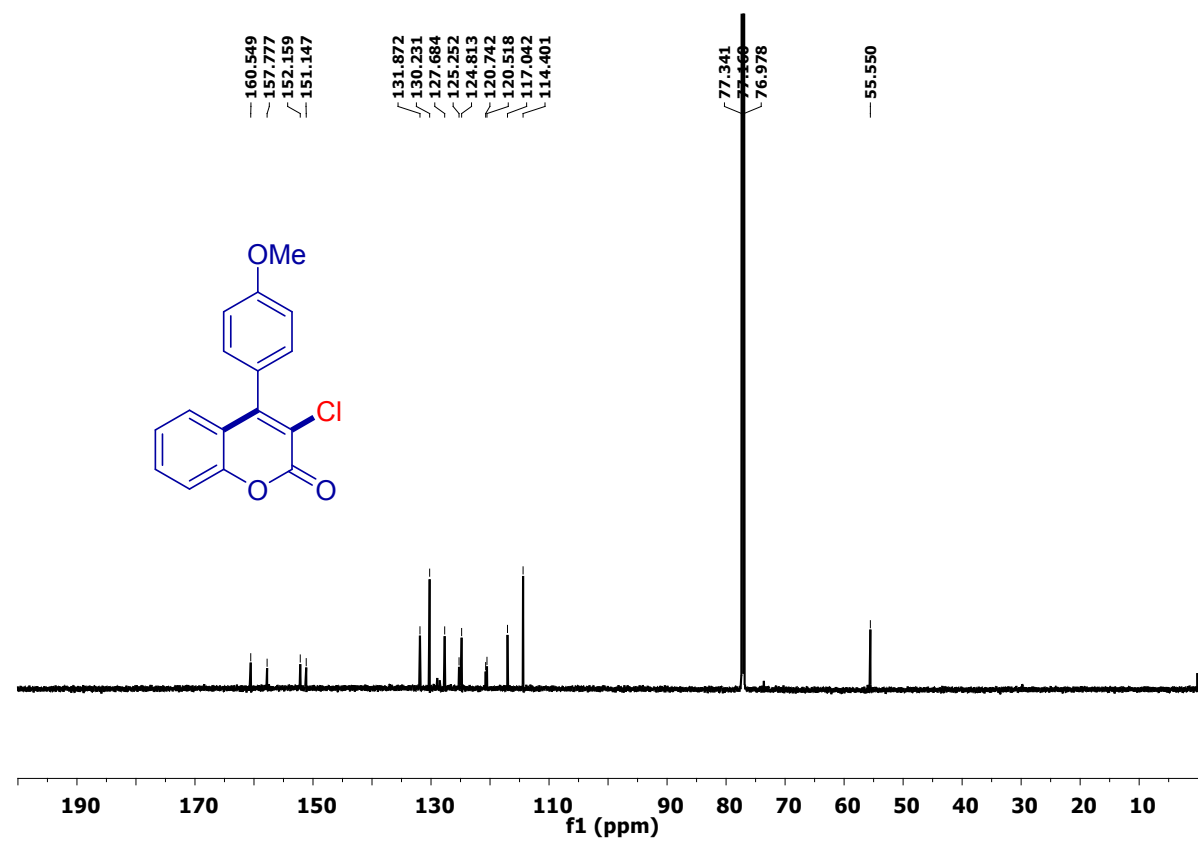

Fig. S66. ${ }^{13} \mathrm{C} \mathrm{NMR}\left(175 \mathrm{MHz}, \mathrm{CDCl}_{3}\right.$ ) spectrum of 3-chloro-4-(4-methoxyphenyl)-2H-chromen2-one (4k). 


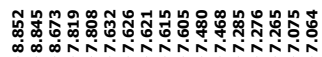
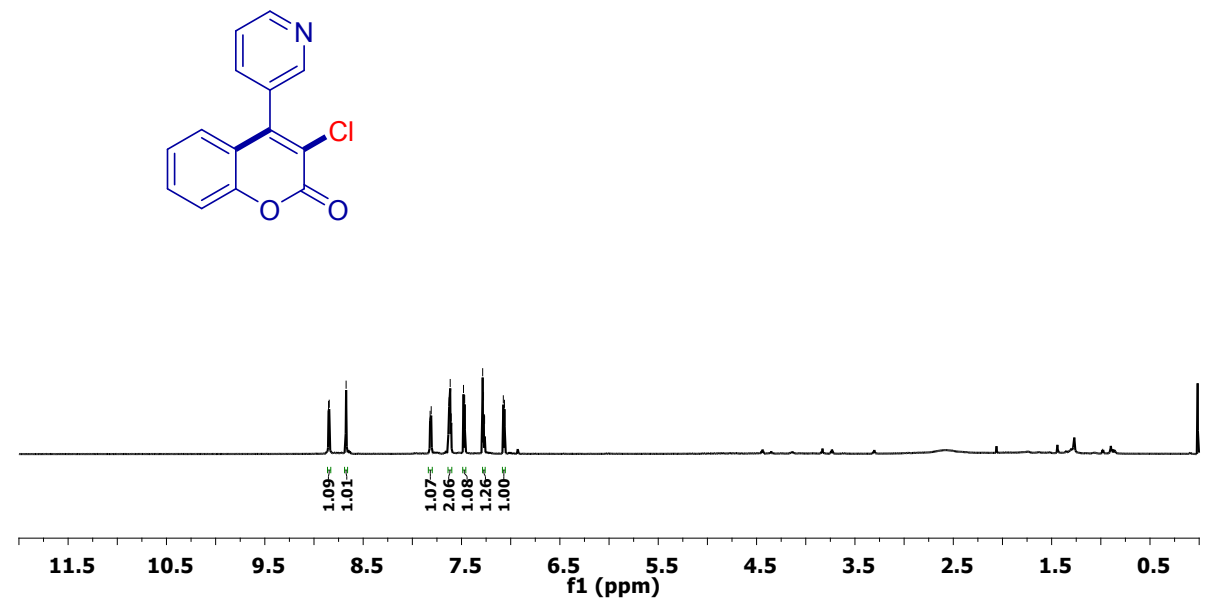

Fig. S67. ${ }^{1} \mathrm{H} \mathrm{NMR}\left(700 \mathrm{MHz}, \mathrm{CDCl}_{3}\right)$ spectrum of 3-chloro-4-(pyridin-3-yl)-2H-chromen-2-one (4I).

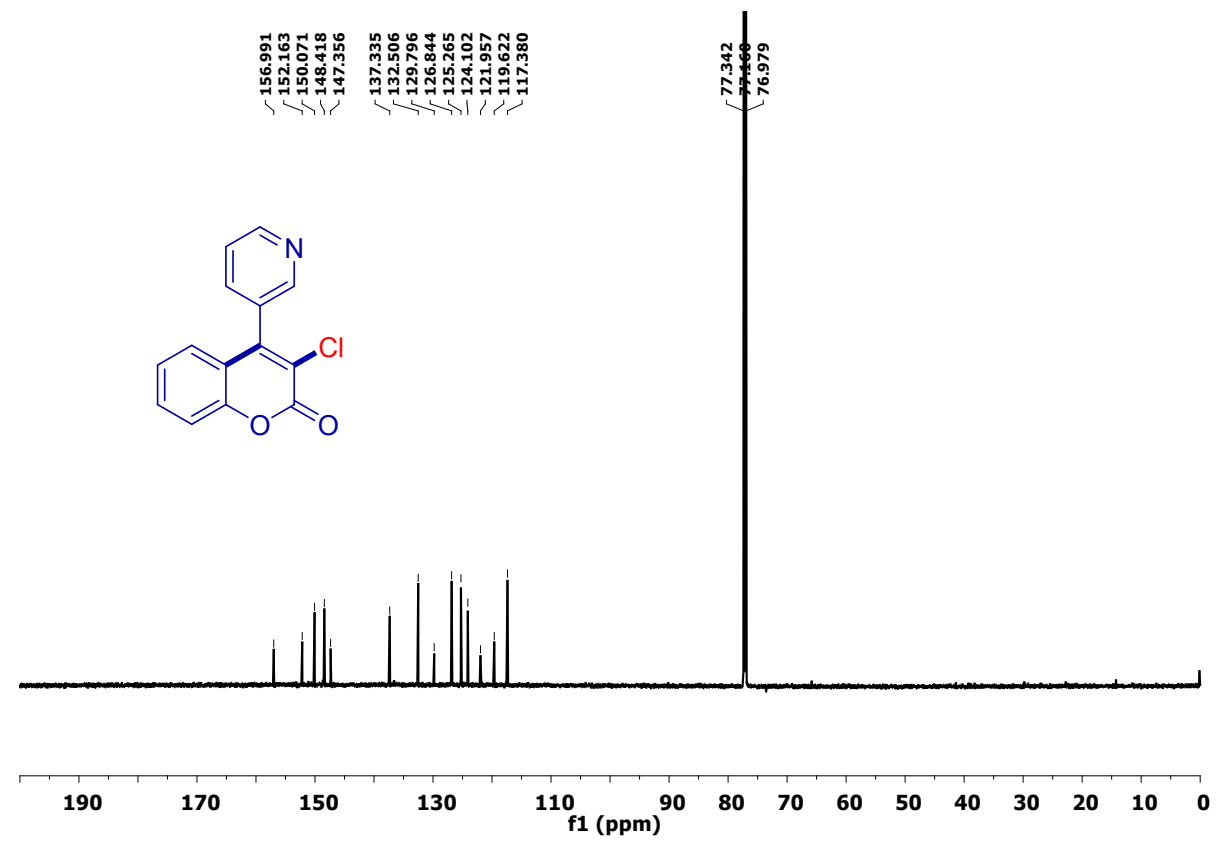

Fig. S68. ${ }^{13} \mathrm{C} \mathrm{NMR}\left(175 \mathrm{MHz}, \mathrm{CDCl}_{3}\right)$ spectrum of 3-chloro-4-(pyridin-3-yl)-2H-chromen-2-one (4I). 


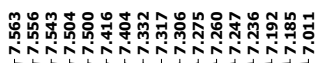

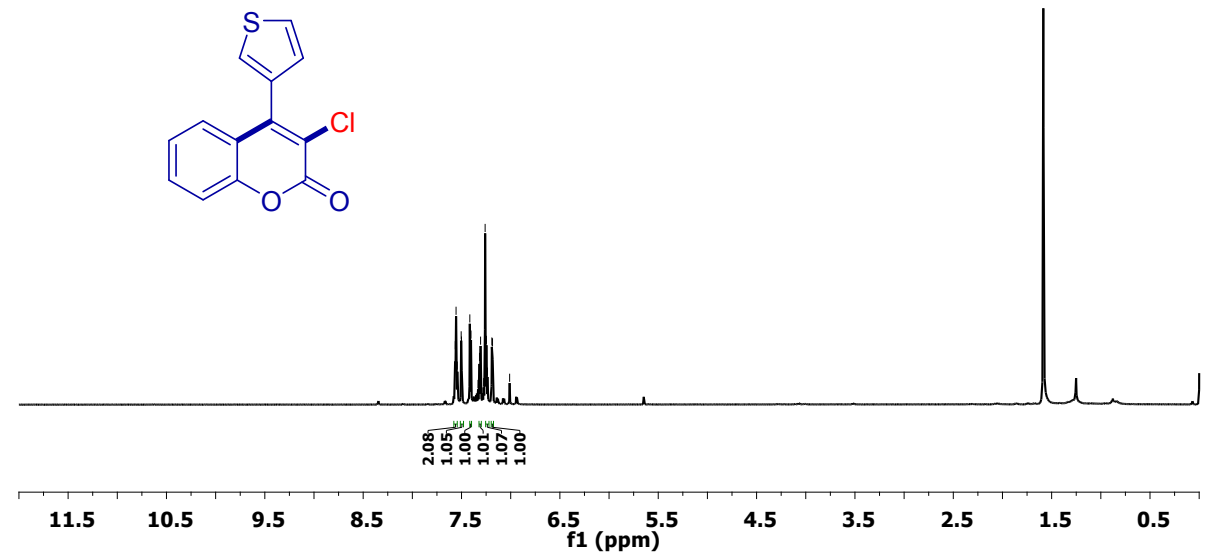

Fig. S69. ${ }^{1} \mathrm{H}$ NMR (700 MHz, $\left.\mathrm{CDCl}_{3}\right)$ spectrum of 3-chloro-4-(thiophen-3-yl)-2H-chromen-2one $(4 \mathrm{~m})$.
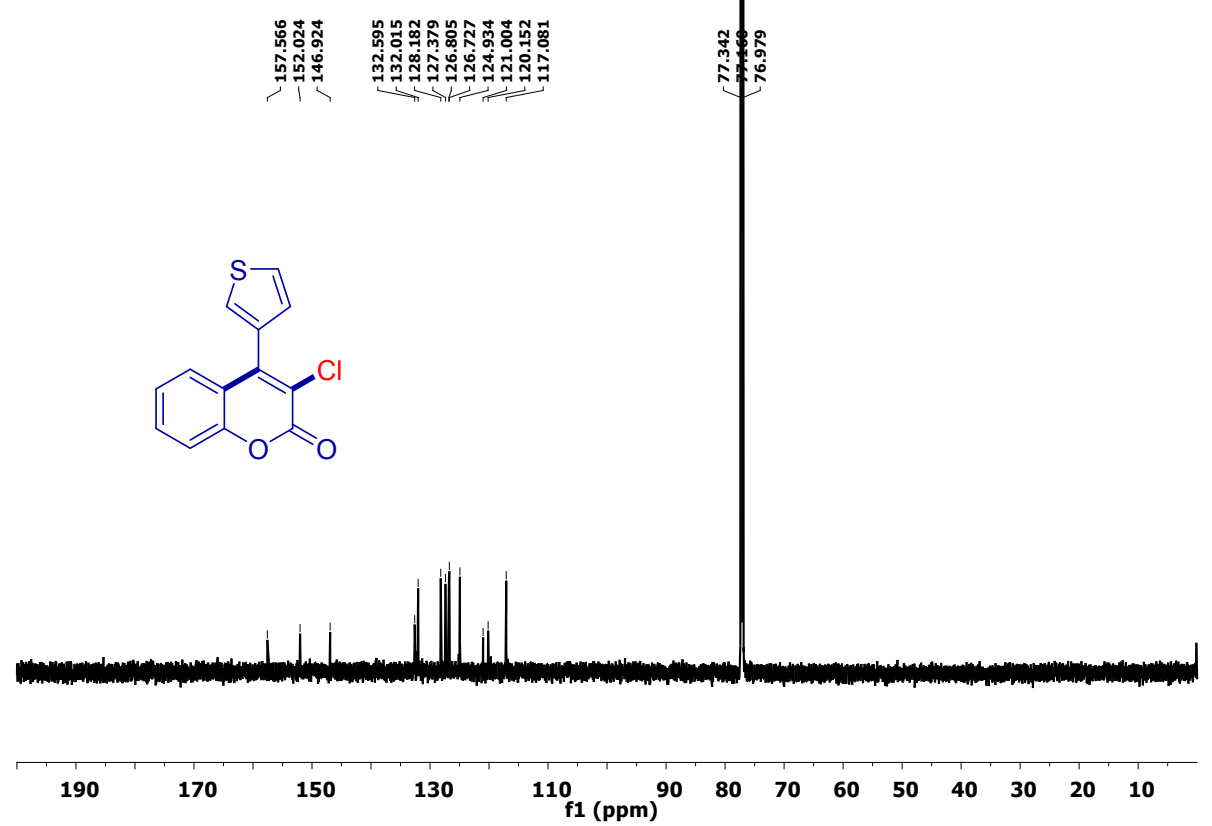

Fig. S70. ${ }^{13} \mathrm{C}$ NMR $\left(175 \mathrm{MHz}, \mathrm{CDCl}_{3}\right)$ spectrum of 3-chloro-4-(thiophen-3-yl)-2H-chromen-2one $(4 \mathrm{~m})$. 


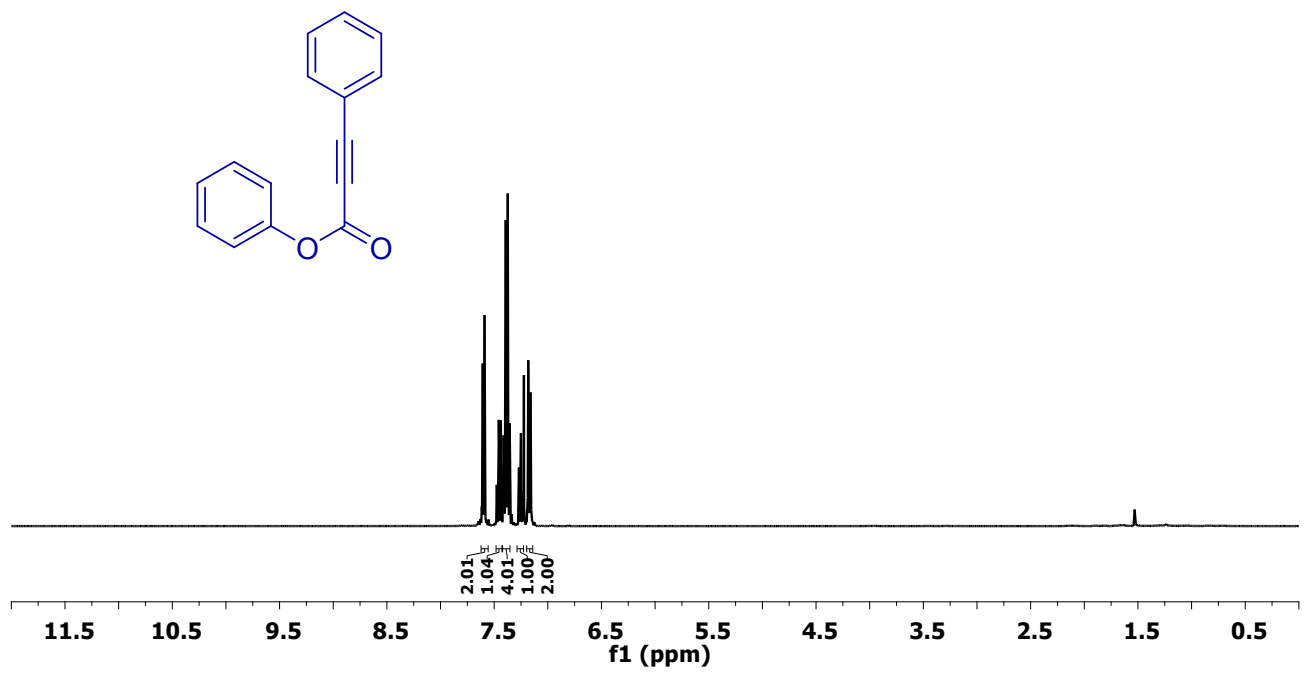

Fig. S71. ${ }^{1} \mathrm{H}$ NMR (400 MHz, $\left.\mathrm{CDCl}_{3}\right)$ spectrum of phenyl 3-phenylpropiolate (1a).

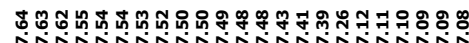

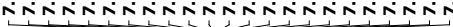

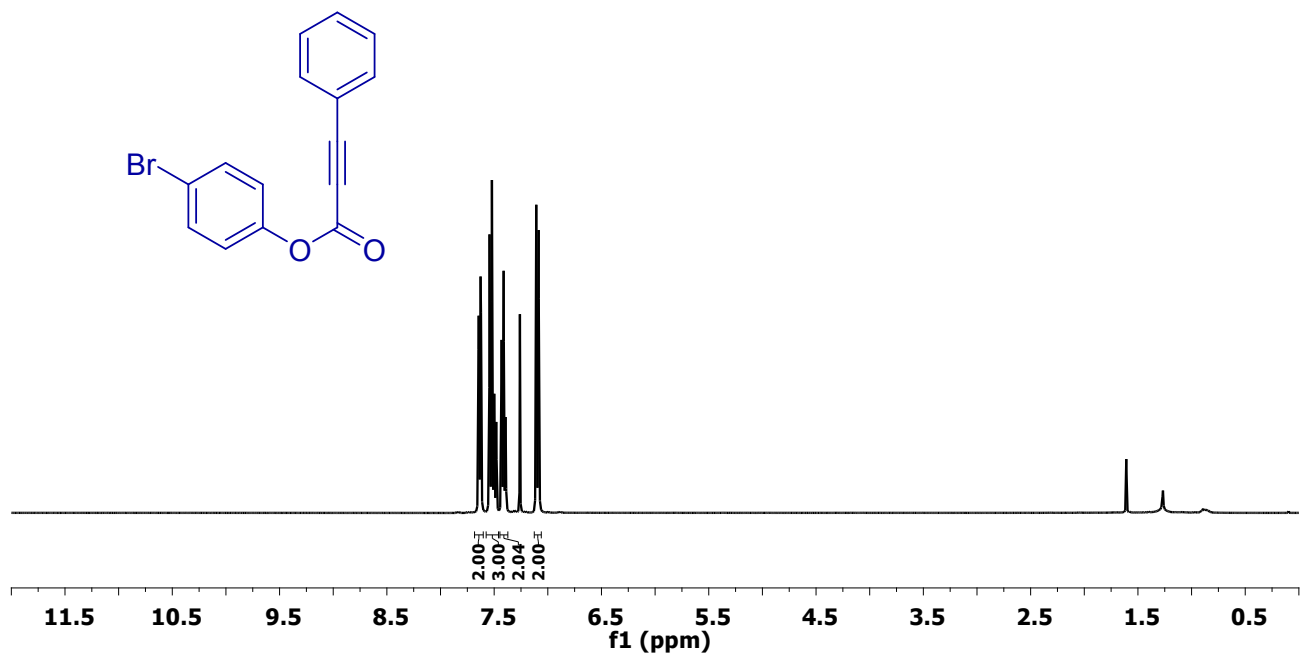

Fig. S72. ${ }^{1} \mathrm{H}$ NMR (400 MHz, $\mathrm{CDCl}_{3}$ ) spectrum of 4-bromophenyl 3-phenylpropiolate (1b). 


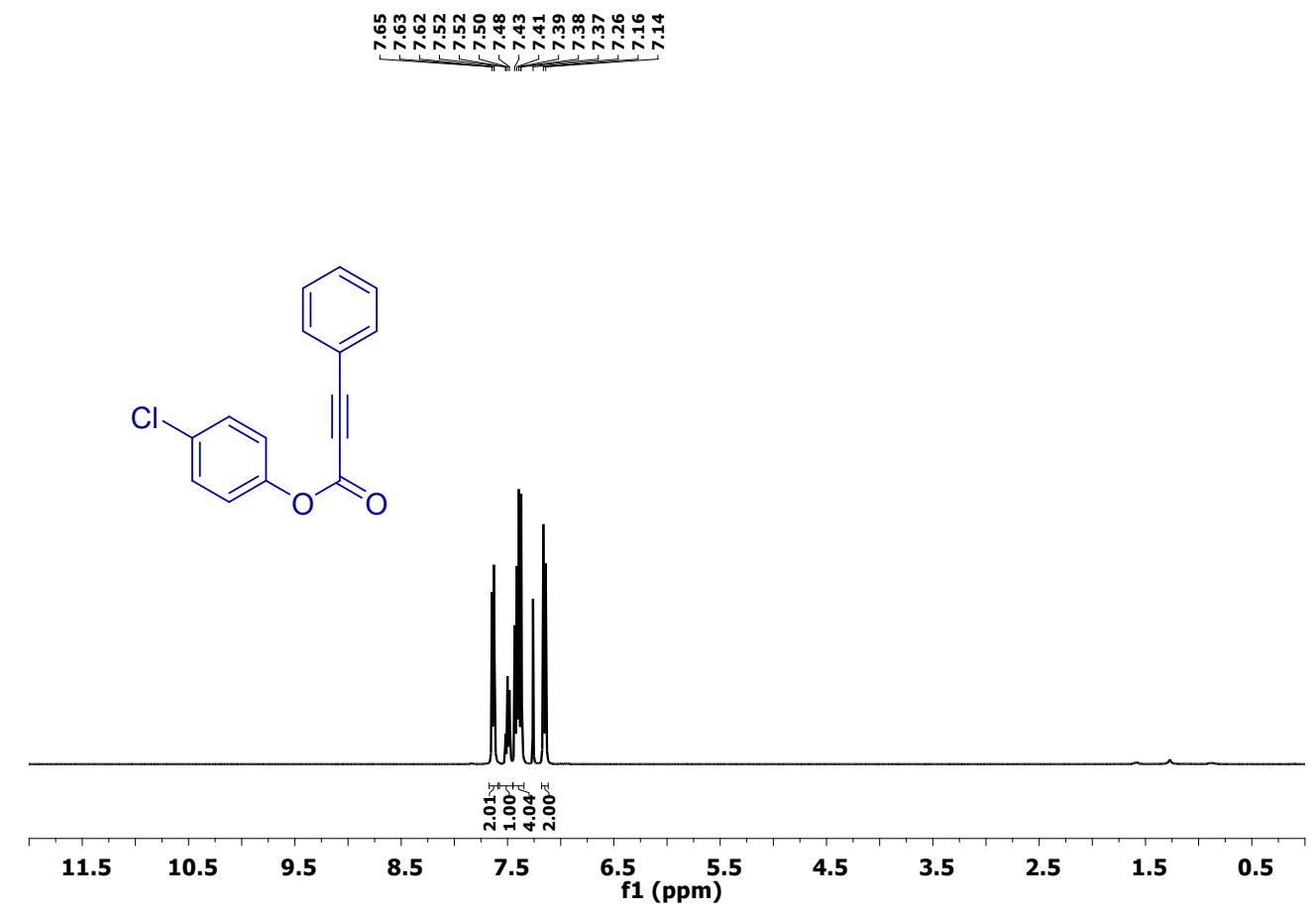

Fig. S73. ${ }^{1} \mathrm{H}$ NMR (400 MHz, $\mathrm{CDCl}_{3}$ ) spectrum of 4-chlorophenyl 3-phenylpropiolate (1c).

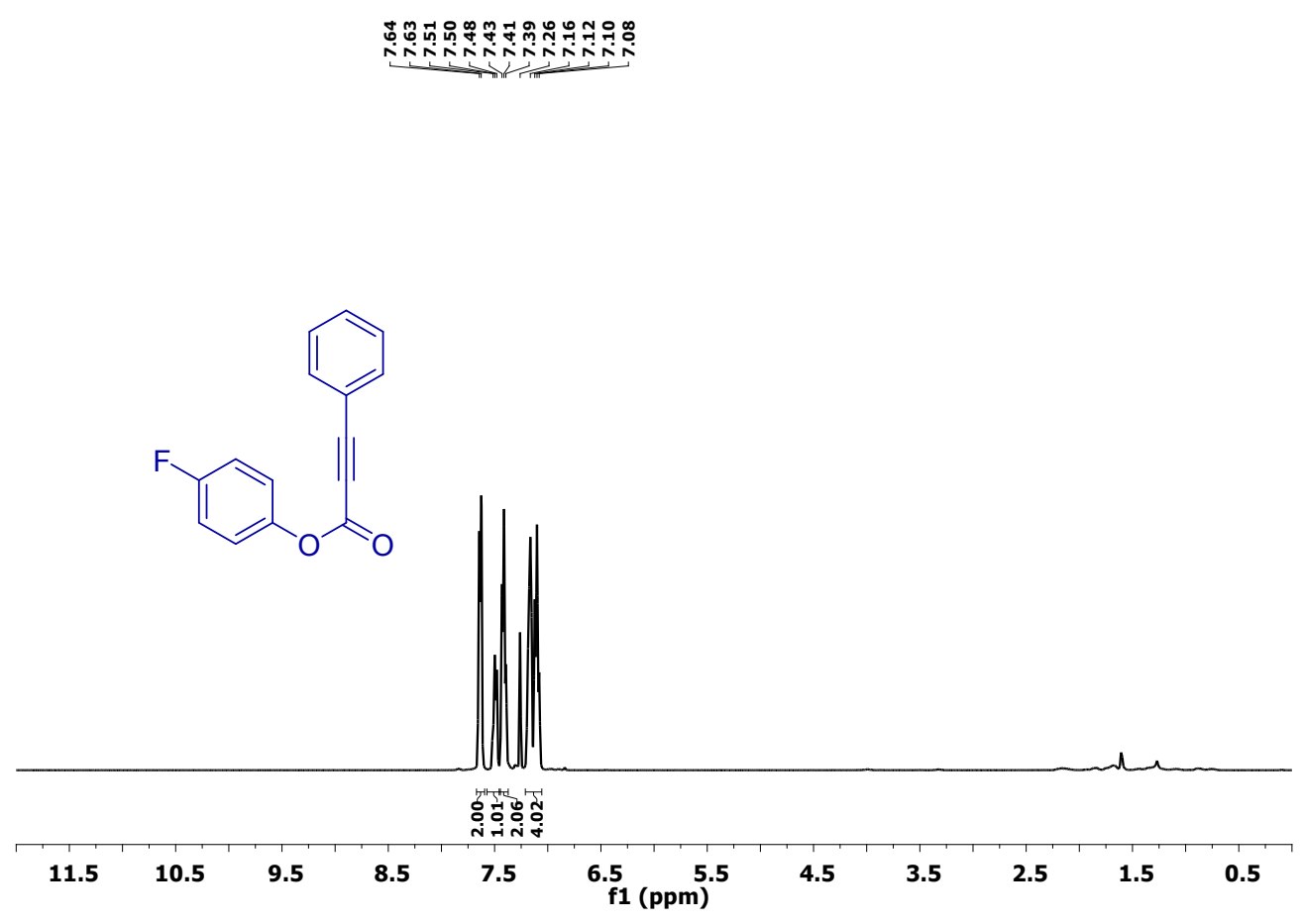

Fig. S74. ${ }^{1} \mathrm{H}$ NMR (400 MHz, $\mathrm{CDCl}_{3}$ ) spectrum of 4-fluorophenyl 3-phenylpropiolate (1d). 


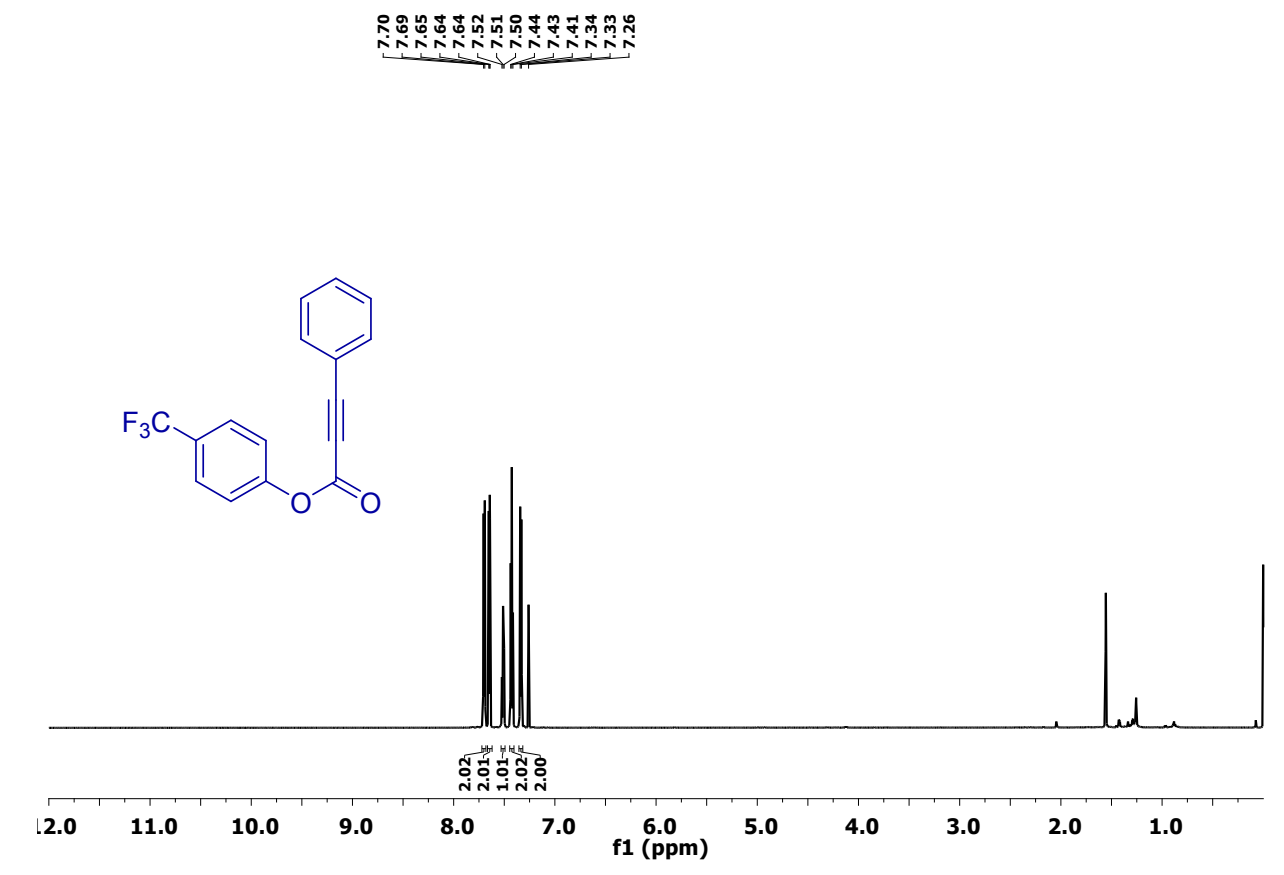

Fig. S75. ${ }^{1} \mathrm{H}$ NMR (700 MHz, $\mathrm{CDCl}_{3}$ ) spectrum of 4-(trifluoromethyl)phenyl 3-phenylpropiolate (1e).

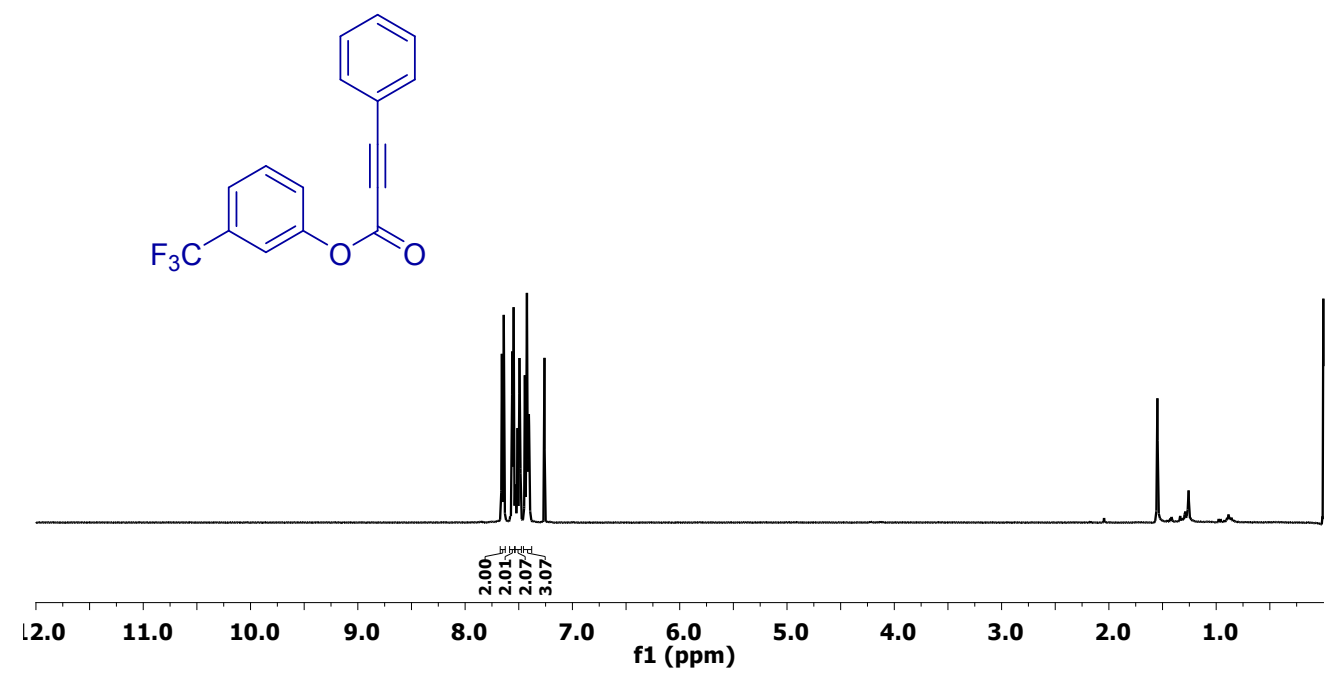

Fig. S76. ${ }^{1} \mathrm{H}$ NMR (400 MHz, $\mathrm{CDCl}_{3}$ ) spectrum of 3-(trifluoromethyl)phenyl 3-phenylpropiolate (1f). 

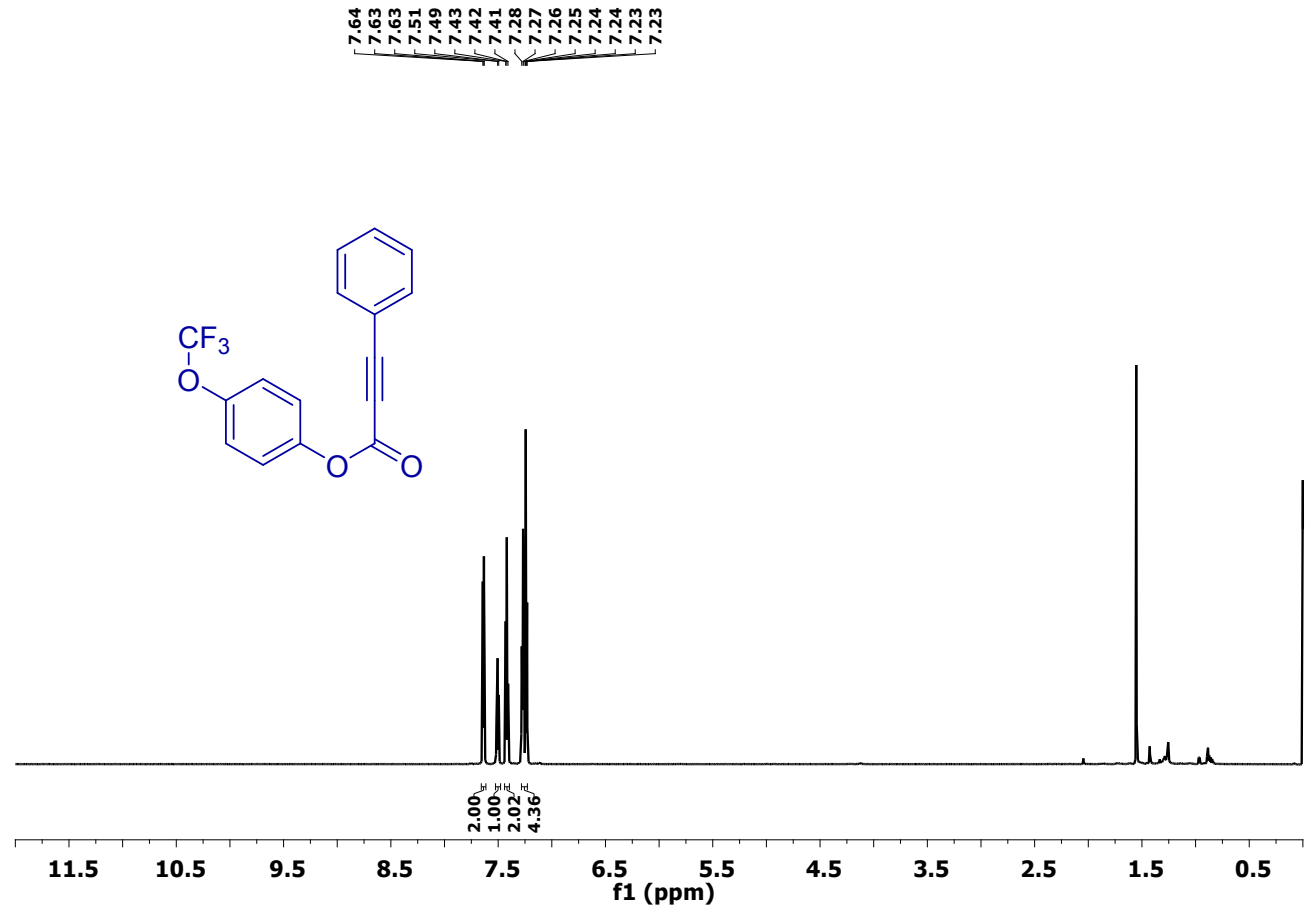

Fig. S77. ${ }^{1} \mathrm{H}$ NMR (700 MHz, $\left.\mathrm{CDCl}_{3}\right)$ spectrum of 4-(trifluoromethoxy)phenyl 3phenylpropiolate (1g).

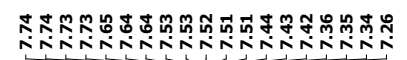<smiles>N#Cc1ccc(OC(=O)C#Cc2ccccc2)cc1</smiles>

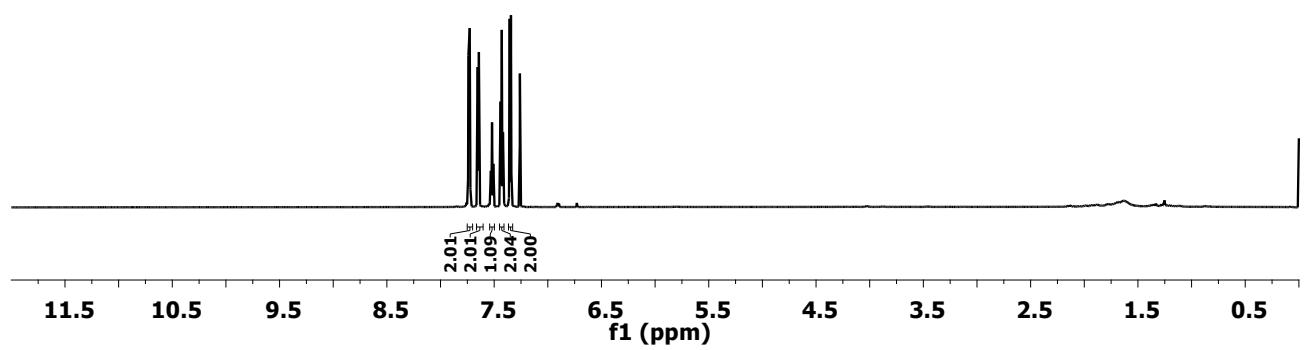

Fig. S78. ${ }^{1} \mathrm{H}$ NMR (700 MHz, $\mathrm{CDCl}_{3}$ ) spectrum of 4-cyanophenyl 3-phenylpropiolate (1h). 

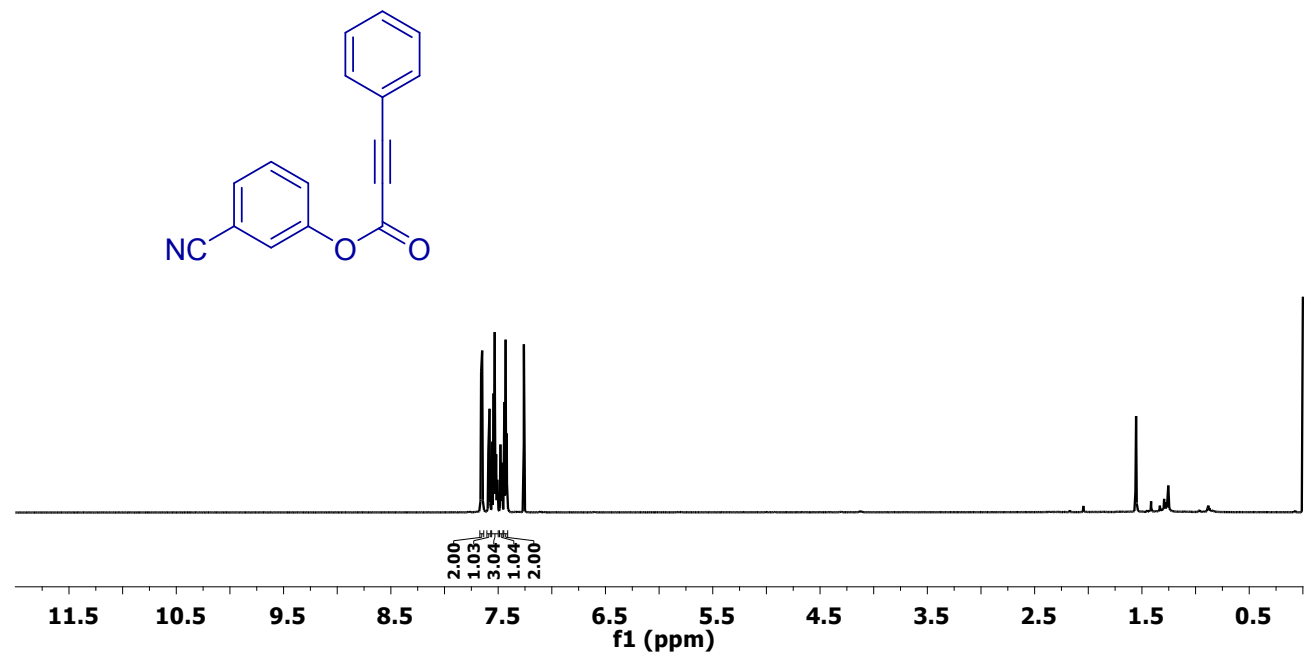

Fig. S79. ${ }^{1} \mathrm{H} \mathrm{NMR}\left(700 \mathrm{MHz}, \mathrm{CDCl}_{3}\right)$ spectrum of 3-cyanophenyl 3-phenylpropiolate (1i).
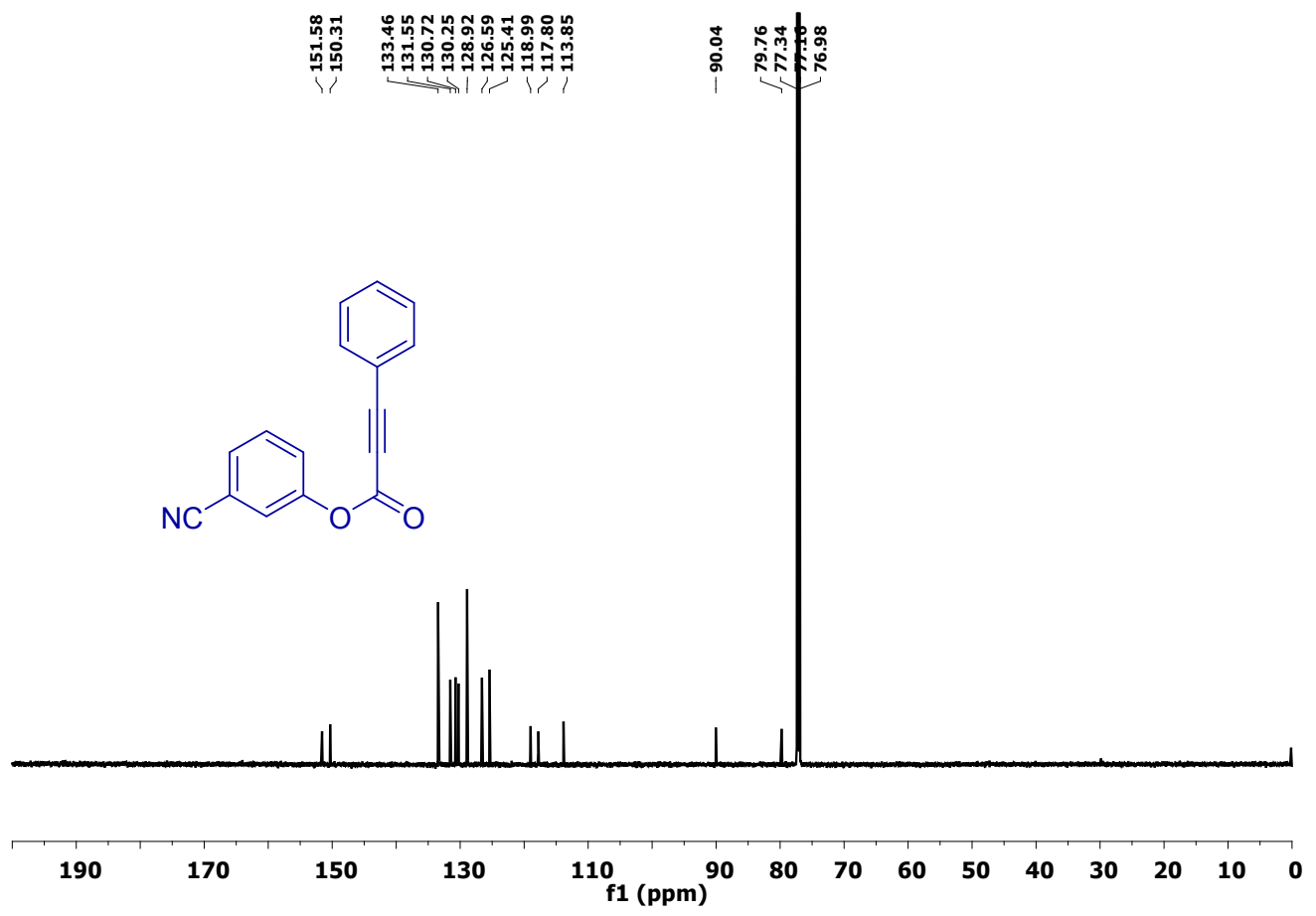

Fig. S80. ${ }^{13} \mathrm{C} \mathrm{NMR}\left(175 \mathrm{MHz}, \mathrm{CDCl}_{3}\right)$ spectrum of 3-cyanophenyl 3-phenylpropiolate (1i). 

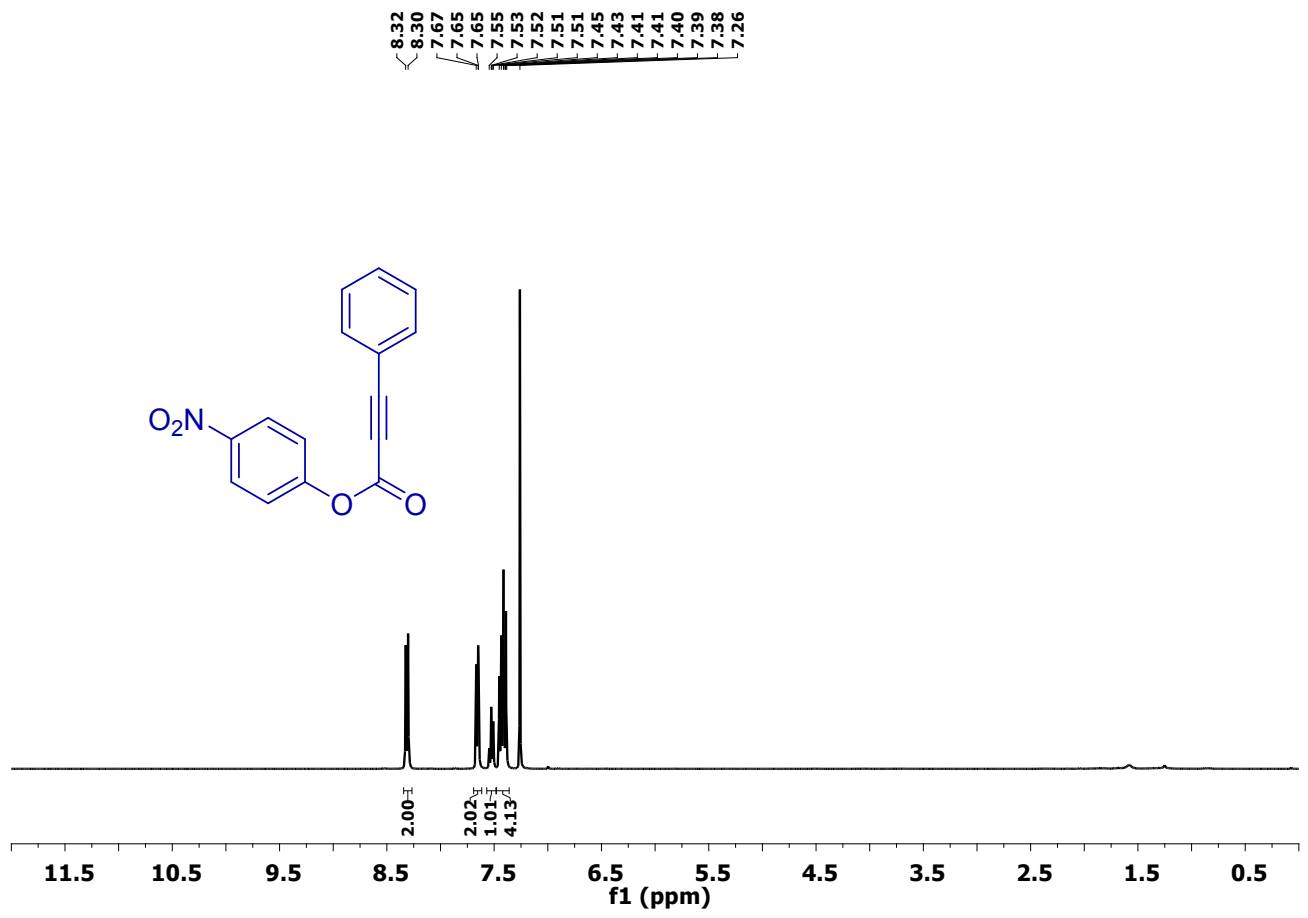

Fig. S81. ${ }^{1} \mathrm{H}$ NMR (400 MHz, $\mathrm{CDCl}_{3}$ ) spectrum of 4-nitrophenyl 3-phenylpropiolate (1j).

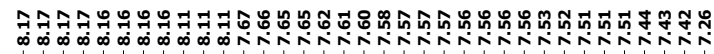

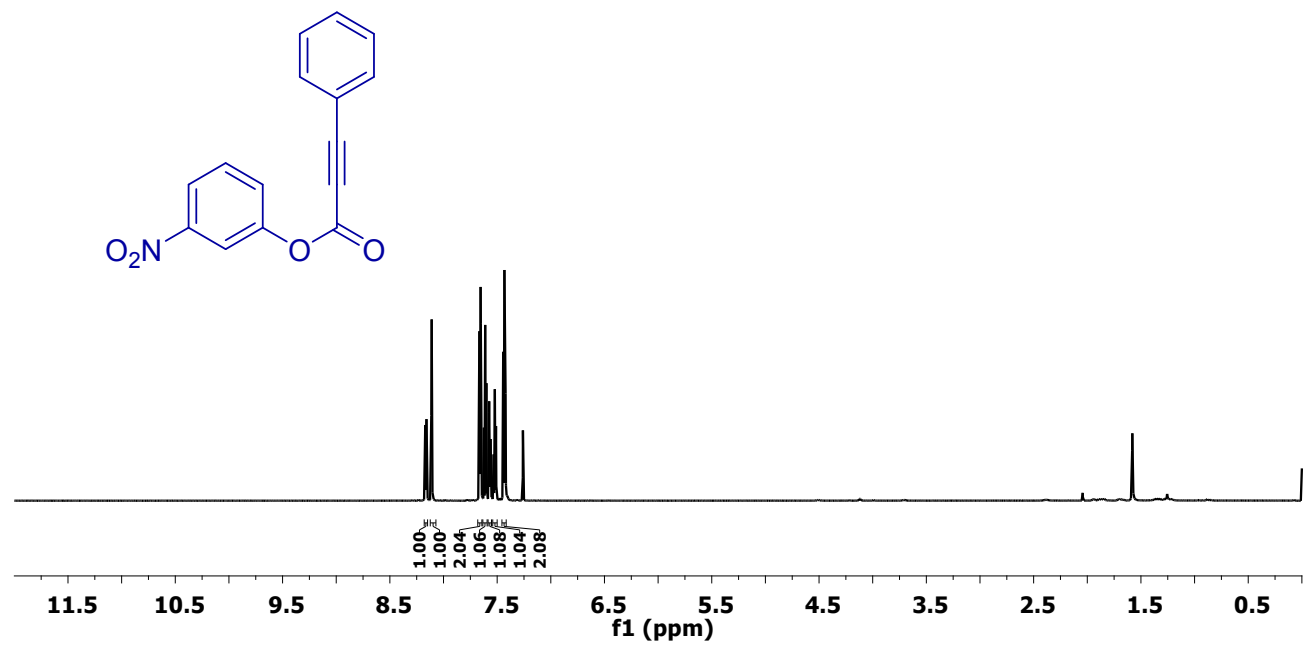

Fig. S82. ${ }^{1} \mathrm{H}$ NMR (700 MHz, $\mathrm{CDCl}_{3}$ ) spectrum of 3-nitrophenyl 3-phenylpropiolate (1k). 


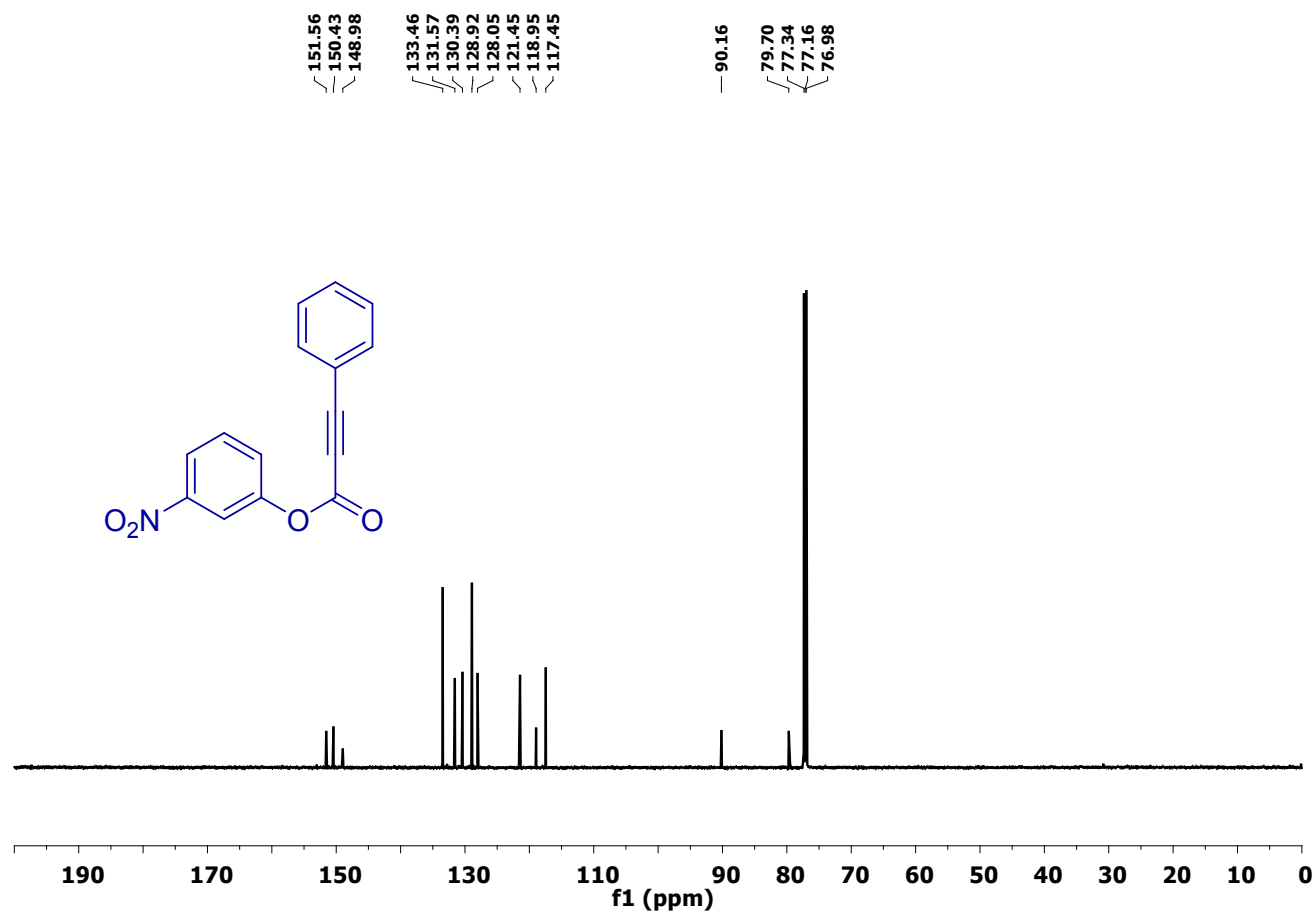

Fig. S83. ${ }^{13} \mathrm{C} \mathrm{NMR}\left(175 \mathrm{MHz}, \mathrm{CDCl}_{3}\right)$ spectrum of 3-nitrophenyl 3-phenylpropiolate (1k). กำดे

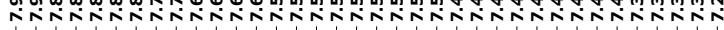

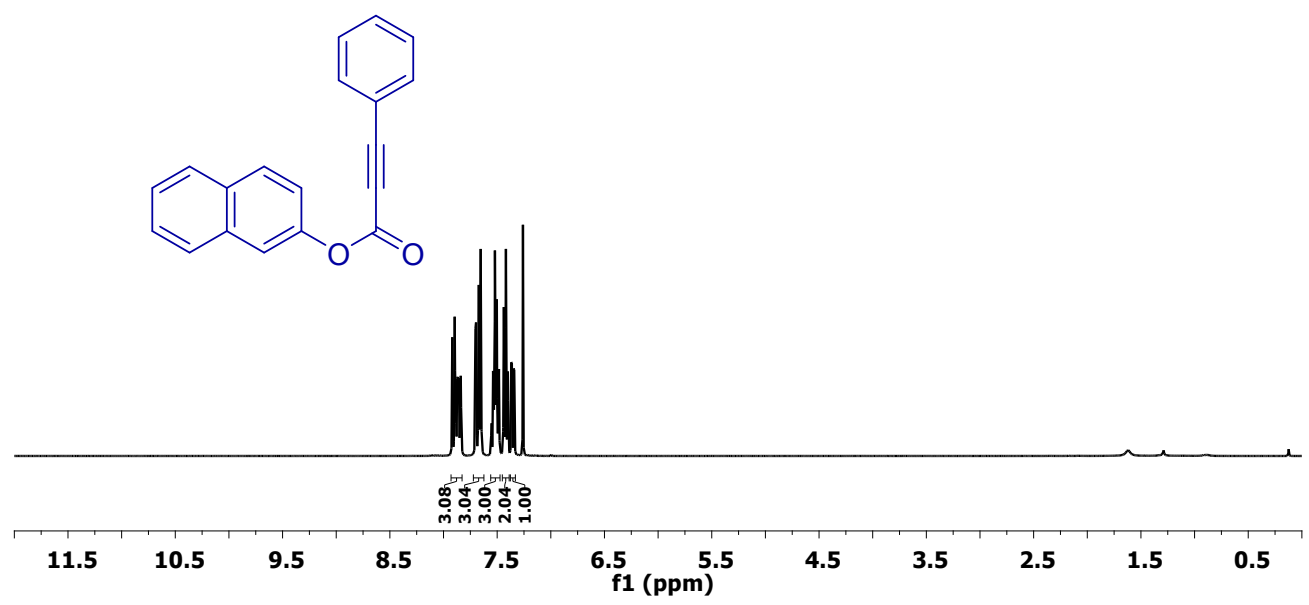

Fig. S84. ${ }^{1} \mathrm{H}$ NMR (400 MHz, $\left.\mathrm{CDCl}_{3}\right)$ spectrum of naphthalen-2-yl 3-phenylpropiolate (1I). 

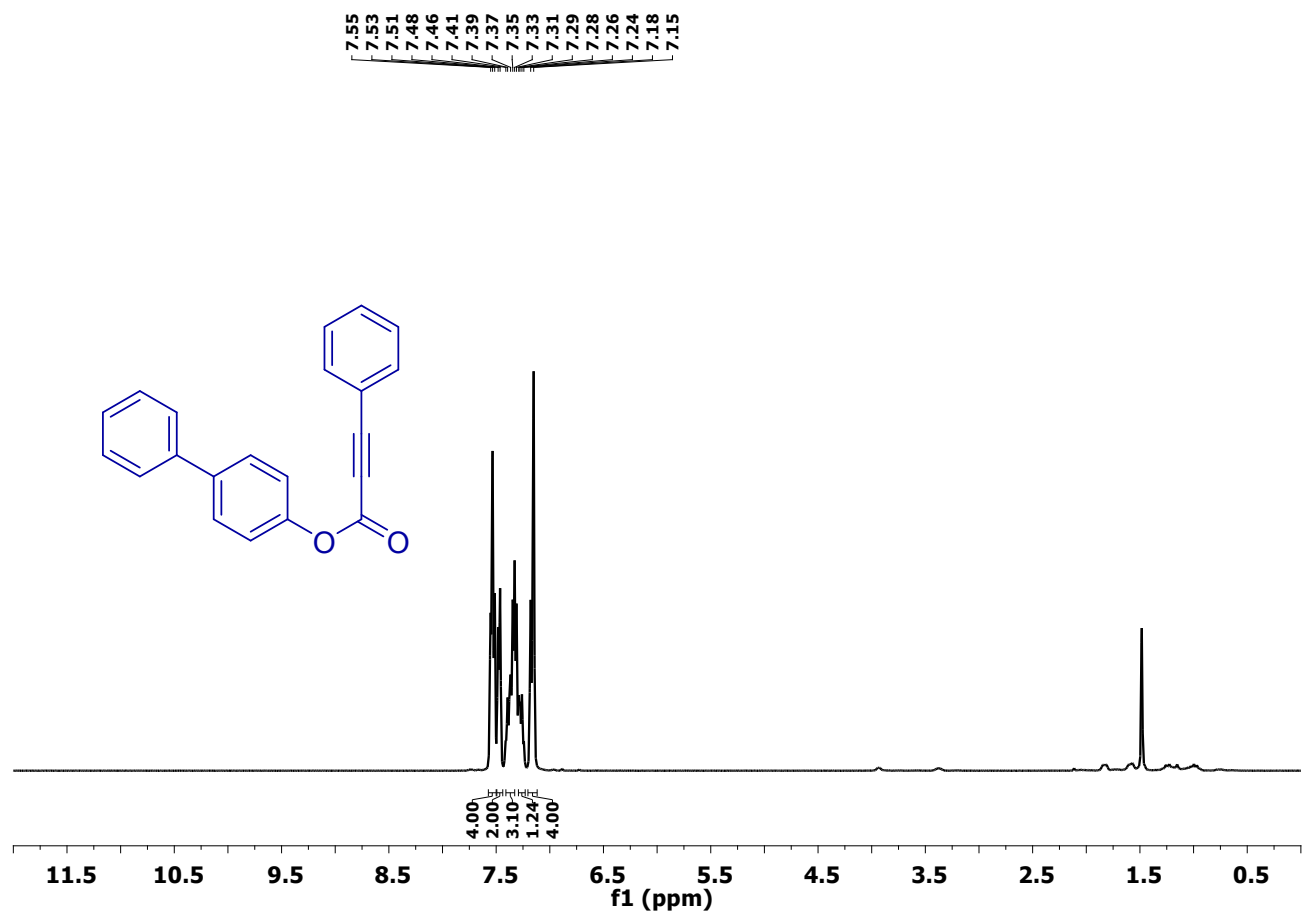

Fig. S85. ${ }^{1} \mathrm{H} \mathrm{NMR}\left(400 \mathrm{MHz}, \mathrm{CDCl}_{3}\right.$ ) spectrum of [1,1'-biphenyl]-4-yl 3-phenylpropiolate (1m).

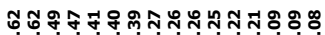

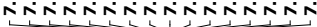

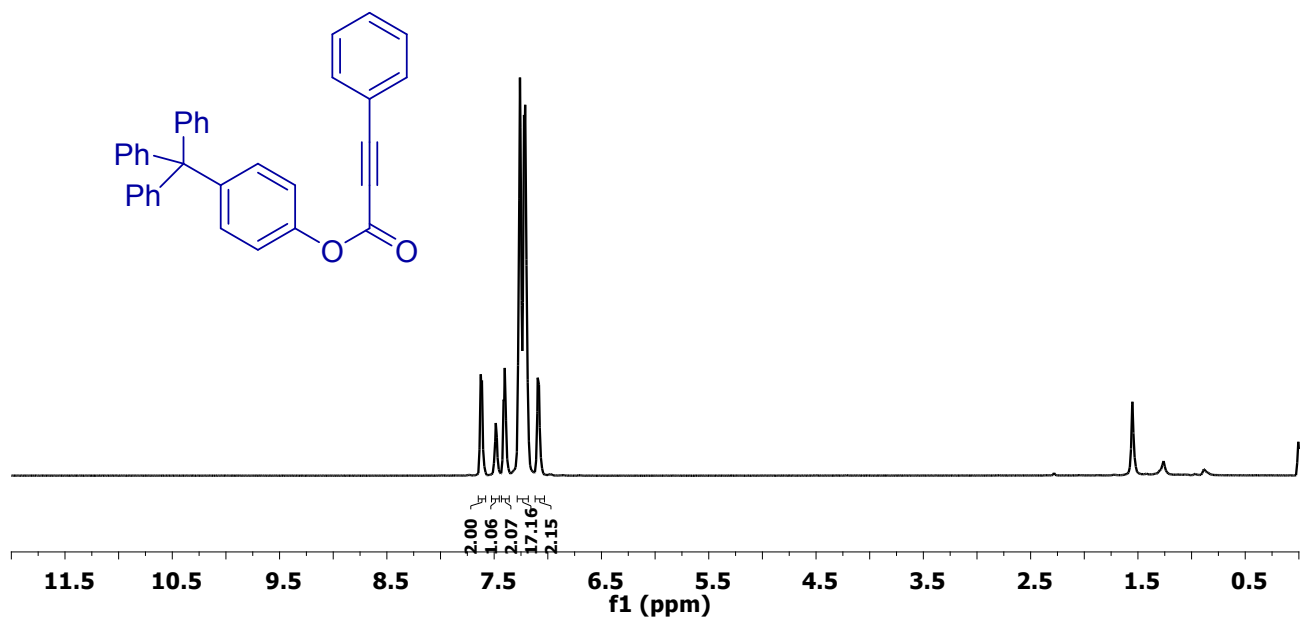

Fig. S86. ${ }^{1} \mathrm{H}$ NMR (700 MHz, $\mathrm{CDCl}_{3}$ ) spectrum of 4-tritylphenyl 3-phenylpropiolate (1n). 


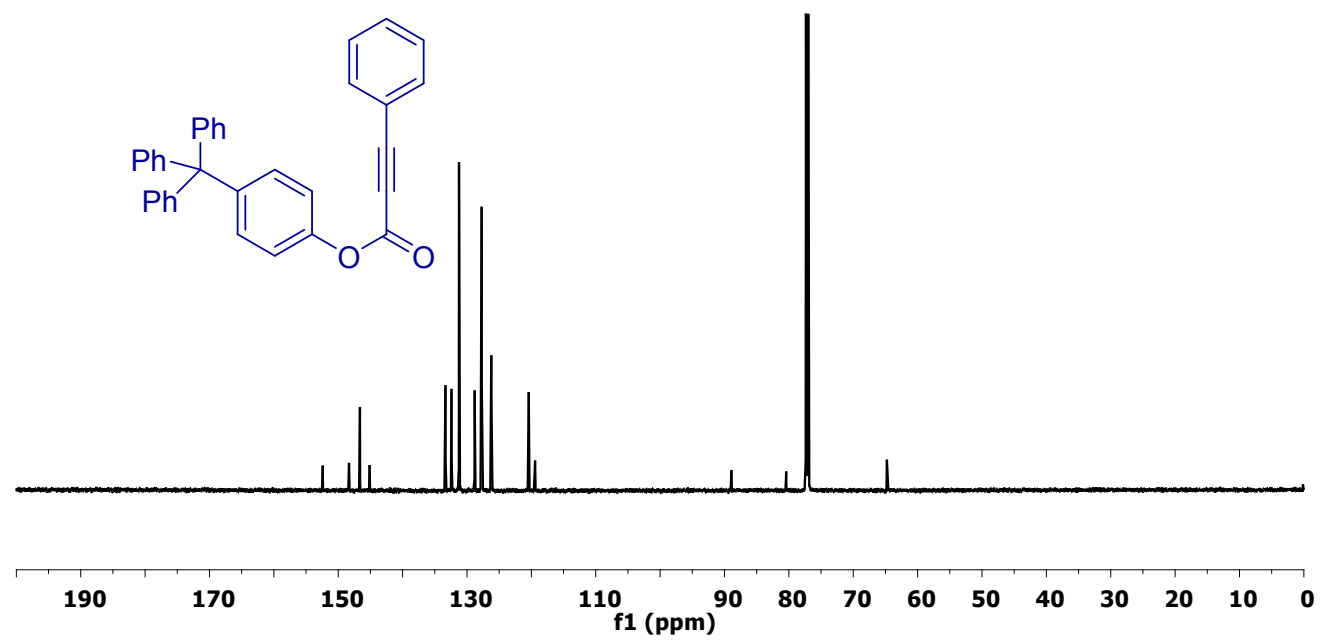

Fig. S87. ${ }^{13} \mathrm{C} \mathrm{NMR}\left(175 \mathrm{MHz}, \mathrm{CDCl}_{3}\right)$ spectrum of 4-tritylphenyl 3-phenylpropiolate (1n).

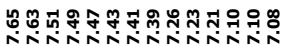

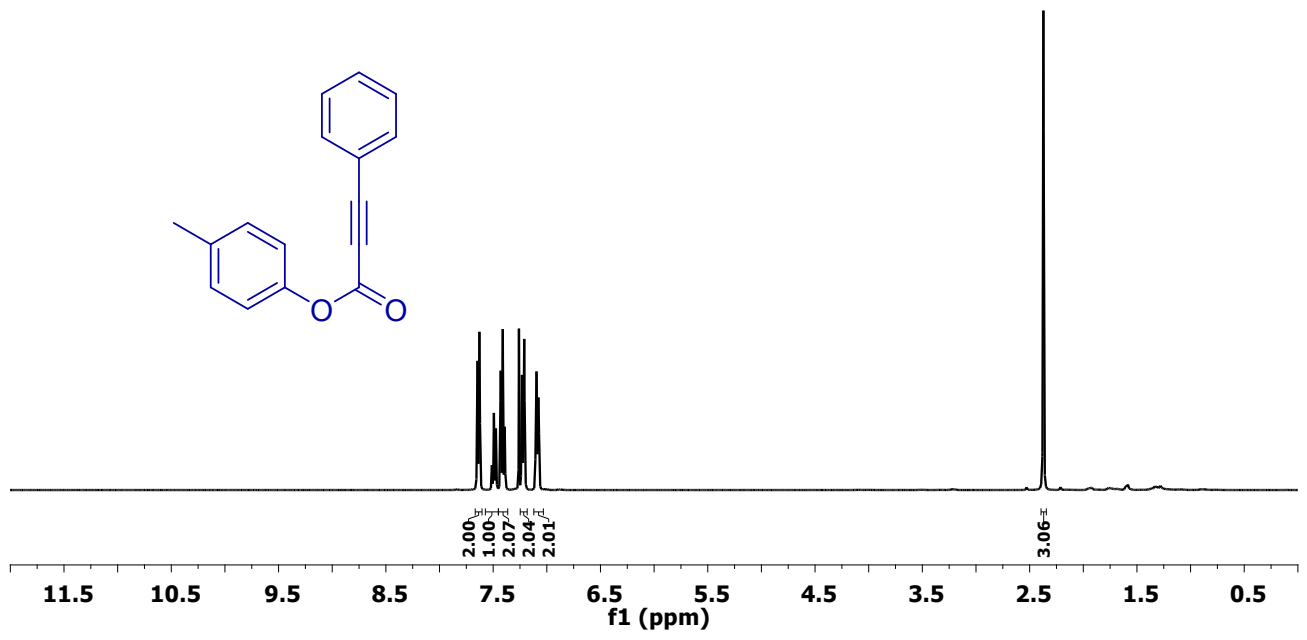

Fig. S88. ${ }^{1} \mathrm{H}$ NMR (400 MHz, $\left.\mathrm{CDCl}_{3}\right)$ spectrum of $p$-tolyl 3-phenylpropiolate (10). 


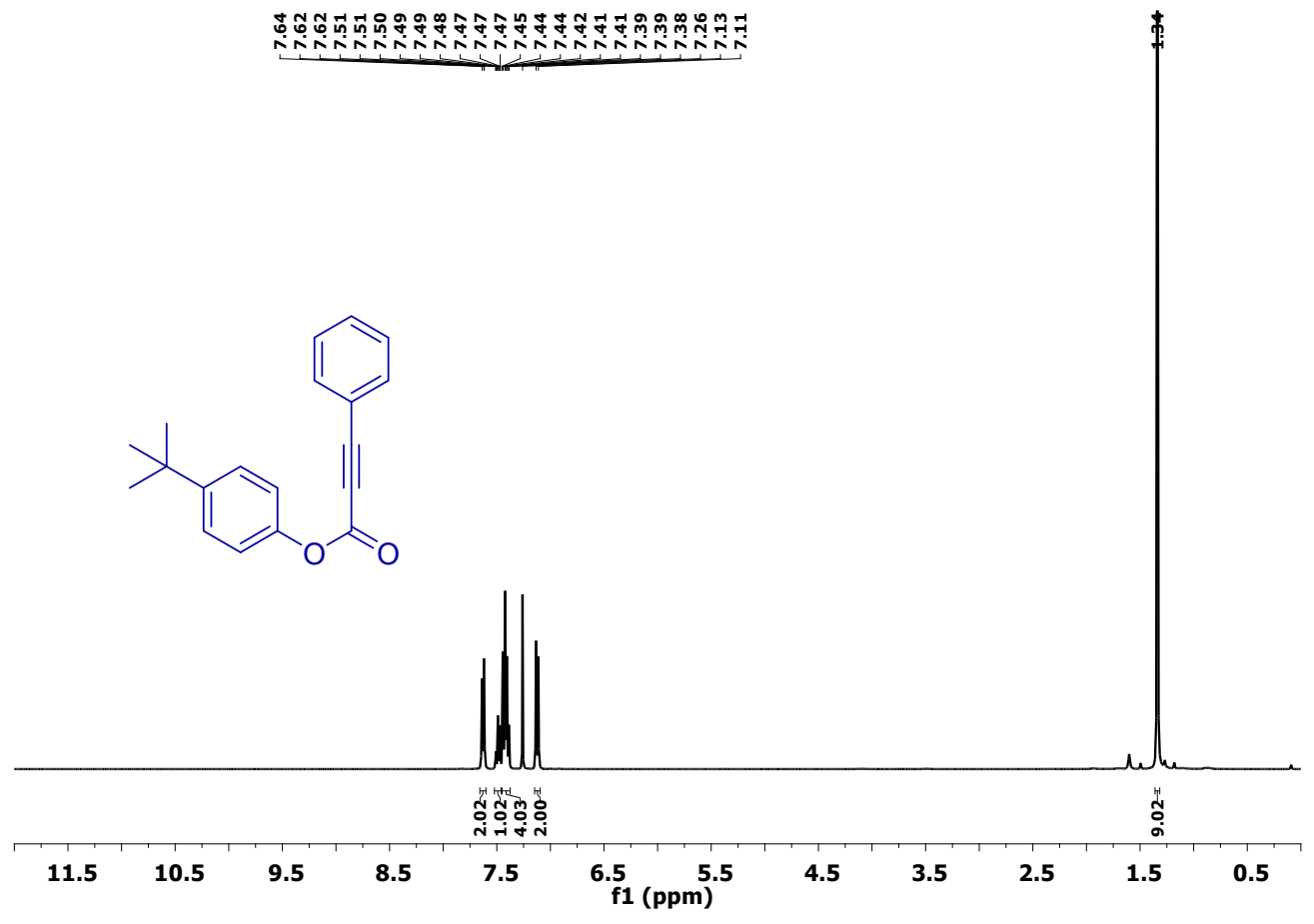

Fig. S89. ${ }^{1} \mathrm{H} \mathrm{NMR}\left(400 \mathrm{MHz}, \mathrm{CDCl}_{3}\right)$ spectrum of 4-(tert-butyl)phenyl 3-phenylpropiolate (1p).
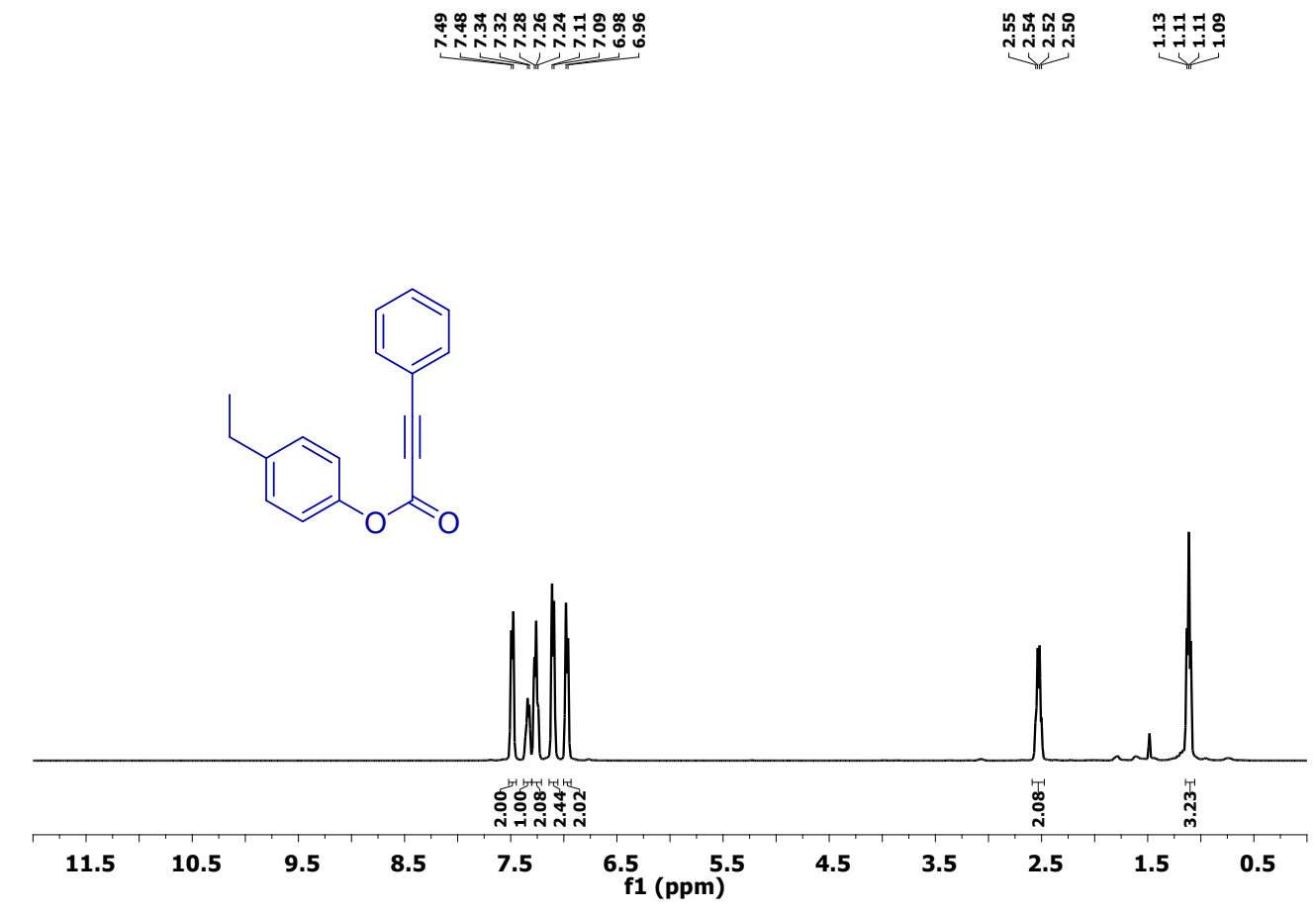

Fig. S90. ${ }^{1} \mathrm{H}$ NMR (400 MHz, $\mathrm{CDCl}_{3}$ ) spectrum of 4-ethylphenyl 3-phenylpropiolate (1q). 


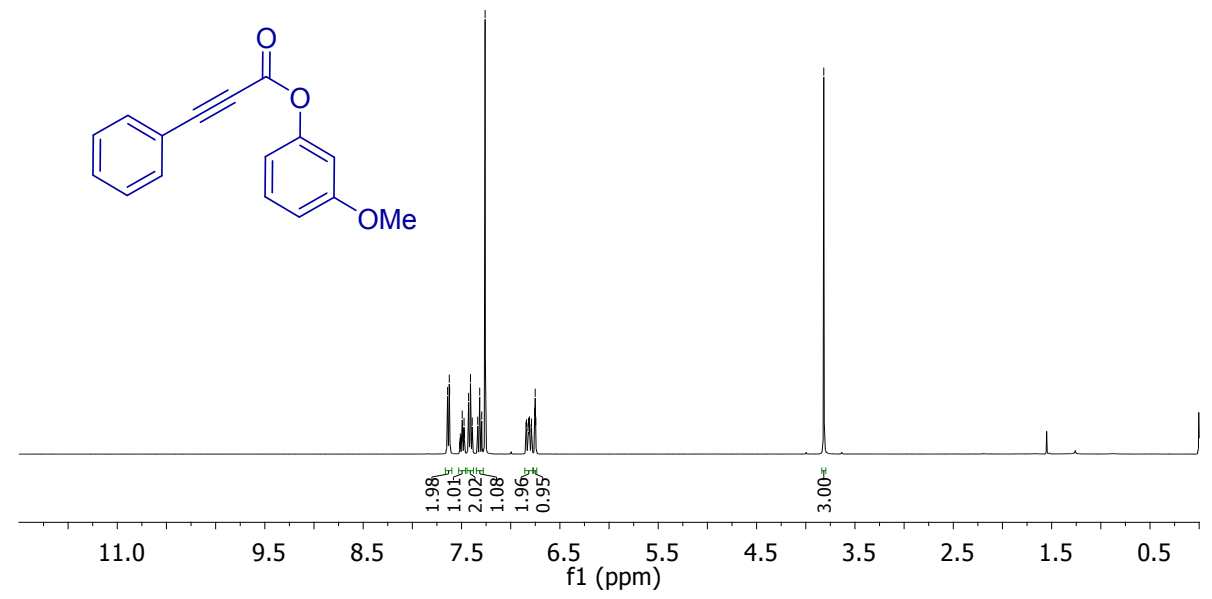

Fig. S91. ${ }^{1} \mathrm{H} \mathrm{NMR}\left(400 \mathrm{MHz}, \mathrm{CDCl}_{3}\right.$ ) spectrum of 3-Methoxyphenyl 3-phenylpropiolate (1s).

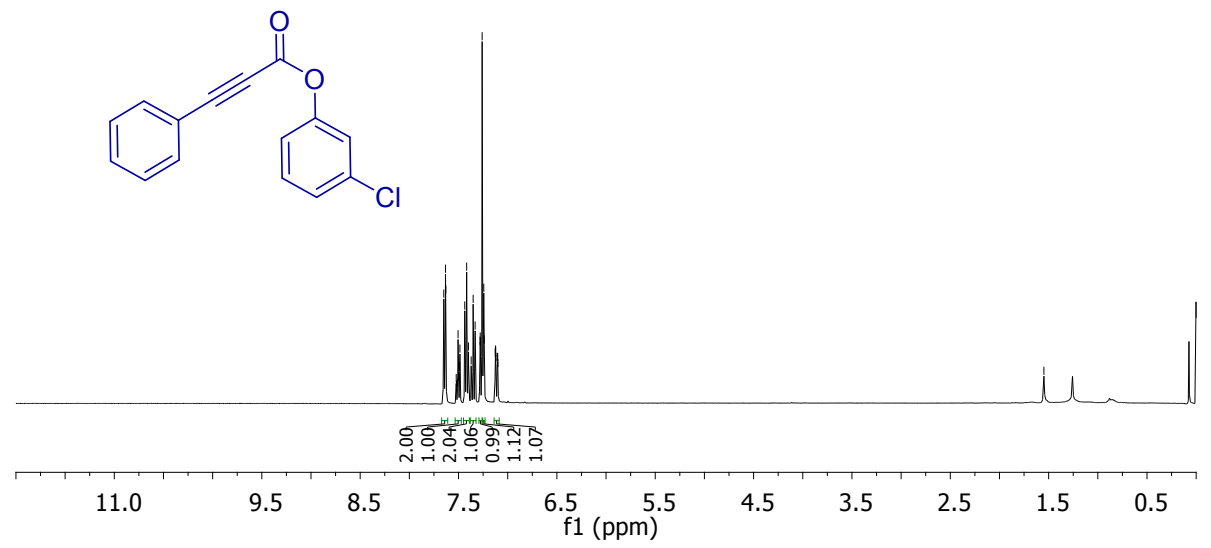

Fig. S92. ${ }^{1} \mathrm{H} \mathrm{NMR}\left(400 \mathrm{MHz}, \mathrm{CDCl}_{3}\right.$ ) spectrum of 3-Chlorophenyl 3-phenylpropiolate (1t). 


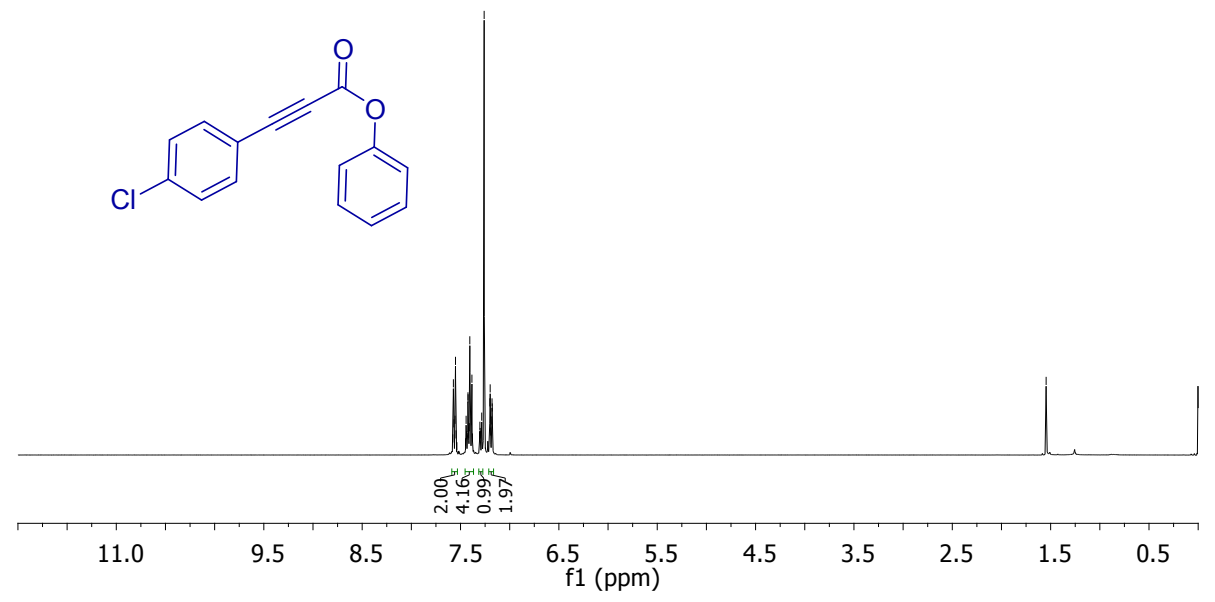

Fig. S93. ${ }^{1} \mathrm{H} \mathrm{NMR}\left(400 \mathrm{MHz}, \mathrm{CDCl}_{3}\right.$ ) spectrum of Phenyl 3-(4-chlorophenyl)propiolate (3a).

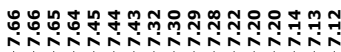
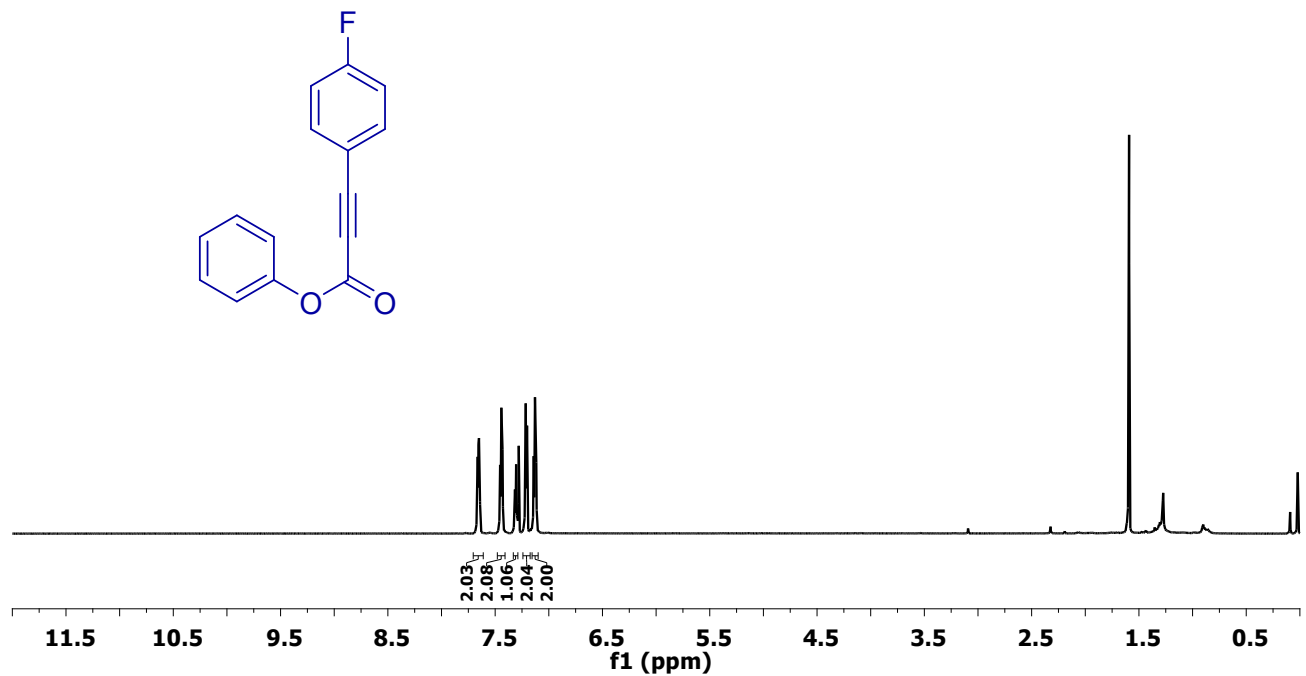

Fig. S94. ${ }^{1} \mathrm{H} \mathrm{NMR}\left(700 \mathrm{MHz}, \mathrm{CDCl}_{3}\right.$ ) spectrum of phenyl 3-(4-fluorophenyl)propiolate (3b). 


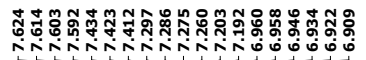
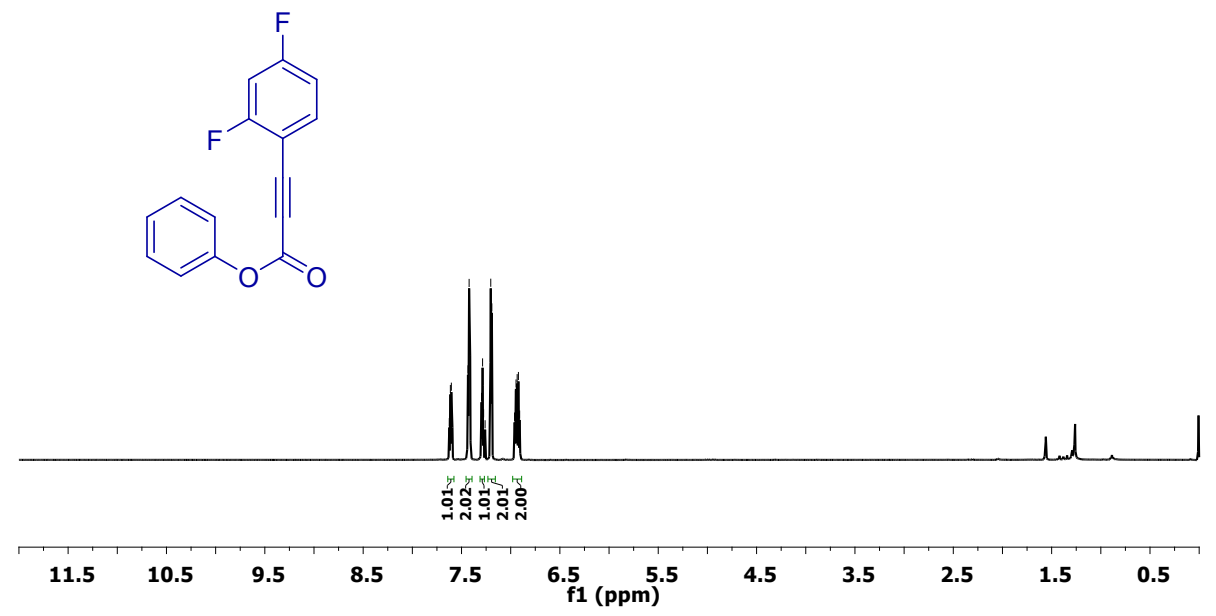

Fig. S95. ${ }^{1} \mathrm{H}$ NMR (700 MHz, $\mathrm{CDCl}_{3}$ ) spectrum of phenyl 3-(2,4-difluorophenyl)propiolate (3c).
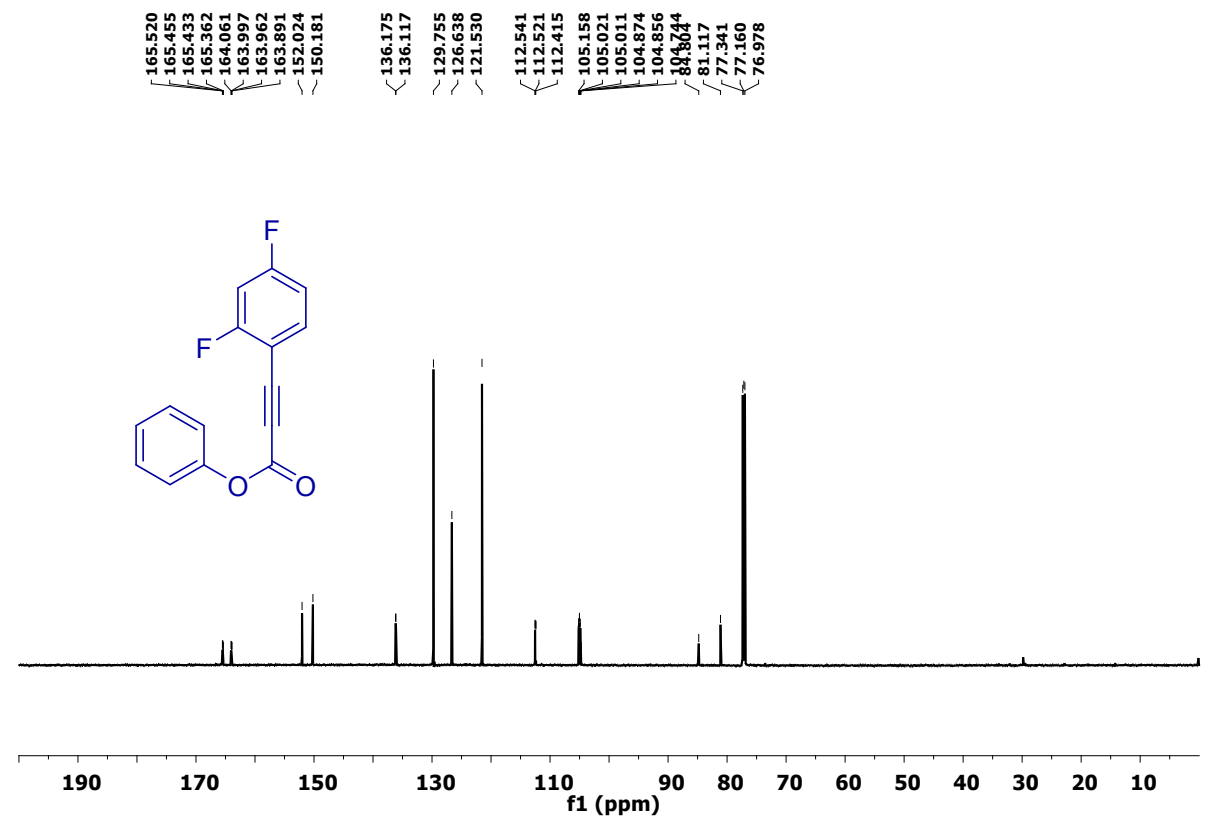

Fig. S96. ${ }^{13} \mathrm{C}$ NMR $\left(175 \mathrm{MHz}, \mathrm{CDCl}_{3}\right)$ spectrum of phenyl 3-(2,4-difluorophenyl)propiolate (3c). 

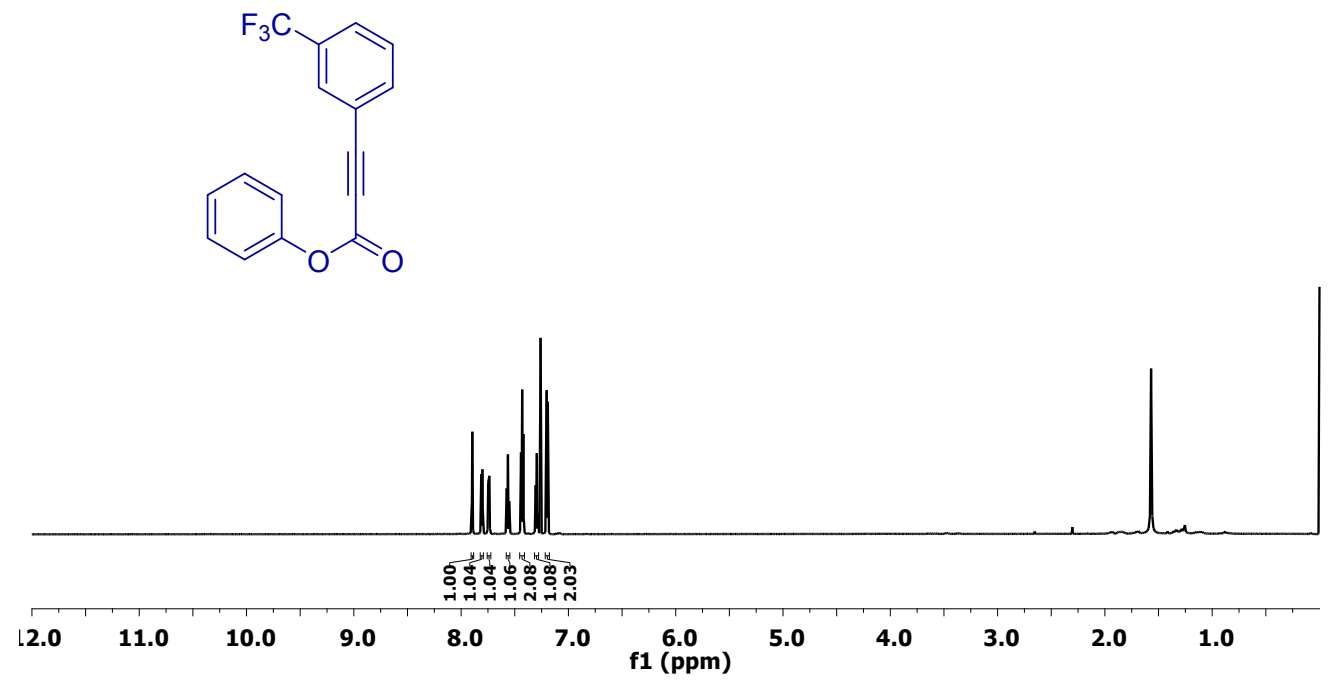

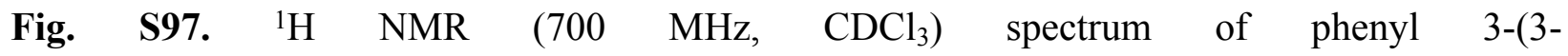
(trifluoromethyl)phenyl)propiolate (3d).

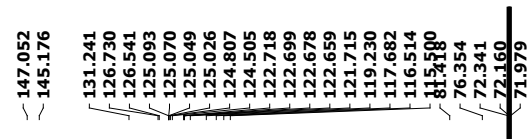<smiles>O=C(/C=C/c1cccc(C(F)(F)F)c1)Oc1ccccc1</smiles>
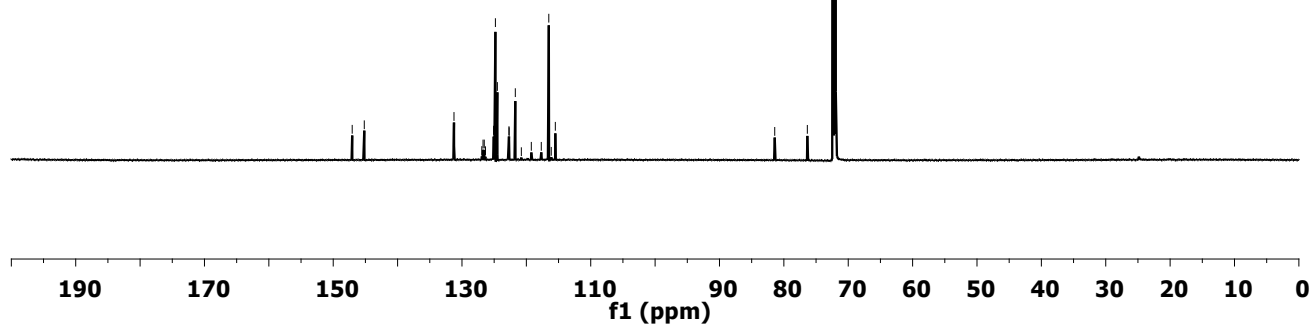

Fig. S98. ${ }^{13} \mathrm{C} \quad \mathrm{NMR} \quad\left(175 \quad \mathrm{MHz}, \quad \mathrm{CDCl}_{3}\right)$ spectrum of phenyl 3-(3(trifluoromethyl)phenyl)propiolate (3d). 


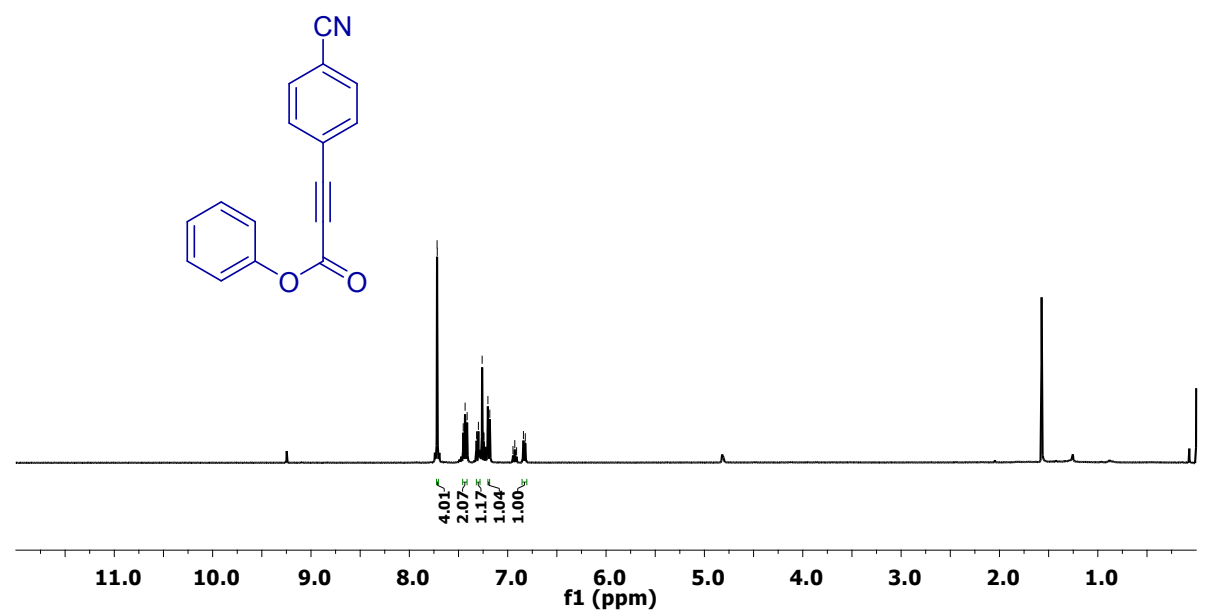

Fig. S99. ${ }^{1} \mathrm{H} \mathrm{NMR}\left(400 \mathrm{MHz}, \mathrm{CDCl}_{3}\right)$ spectrum of phenyl 3-(4-cyanophenyl)propiolate (3e).

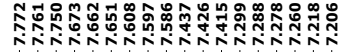<smiles>N#Cc1ccccc1C#CC(=O)Oc1ccccc1</smiles>

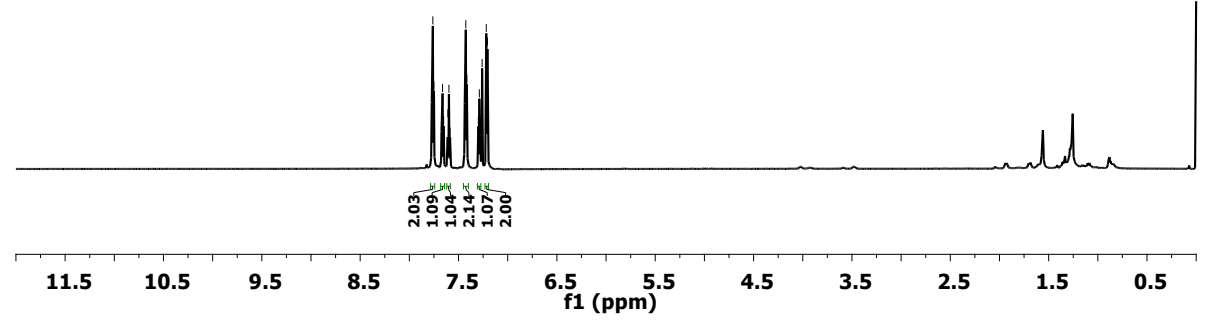

Fig. S100. ${ }^{1} \mathrm{H}$ NMR (700 MHz, $\mathrm{CDCl}_{3}$ ) spectrum of phenyl 3-(2-cyanophenyl)propiolate (3f). 


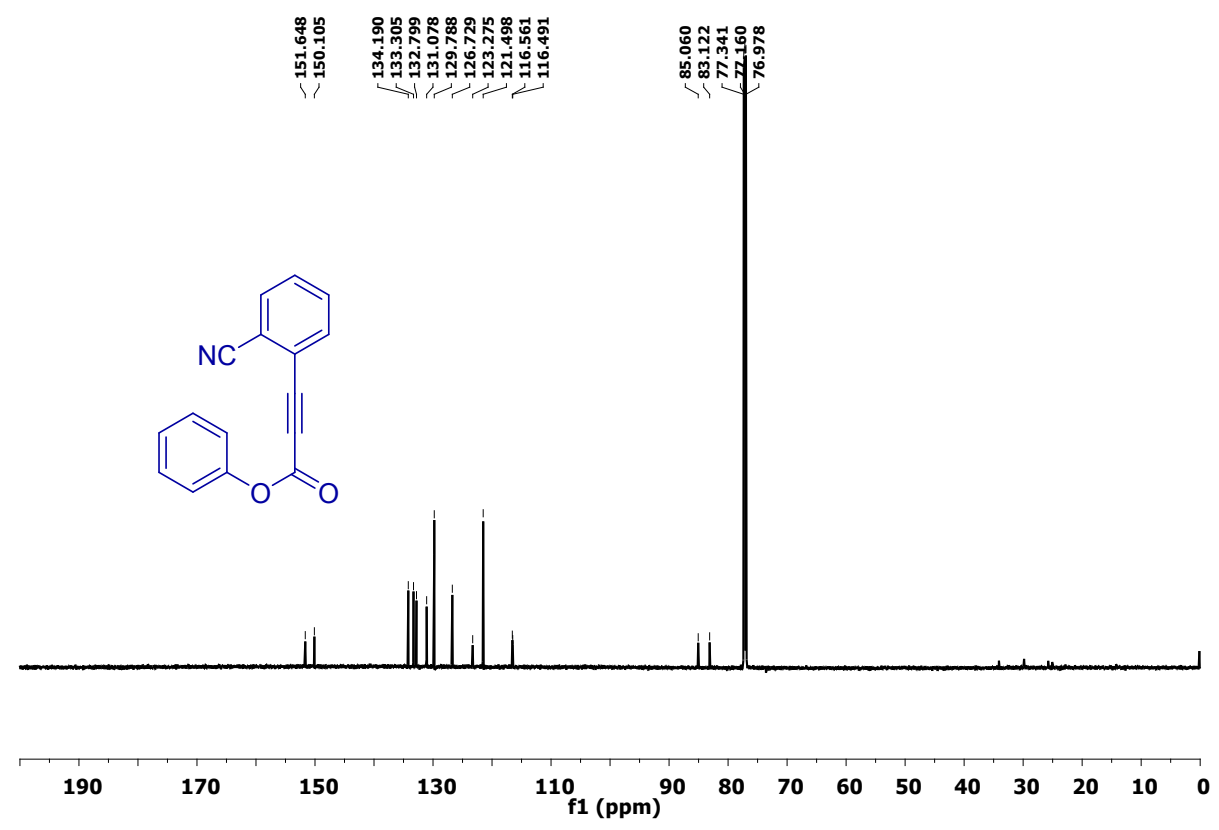

Fig. S101. ${ }^{13} \mathrm{C}$ NMR (175 MHz, $\mathrm{CDCl}_{3}$ ) spectrum of phenyl 3-(2-cyanophenyl)propiolate (3f).

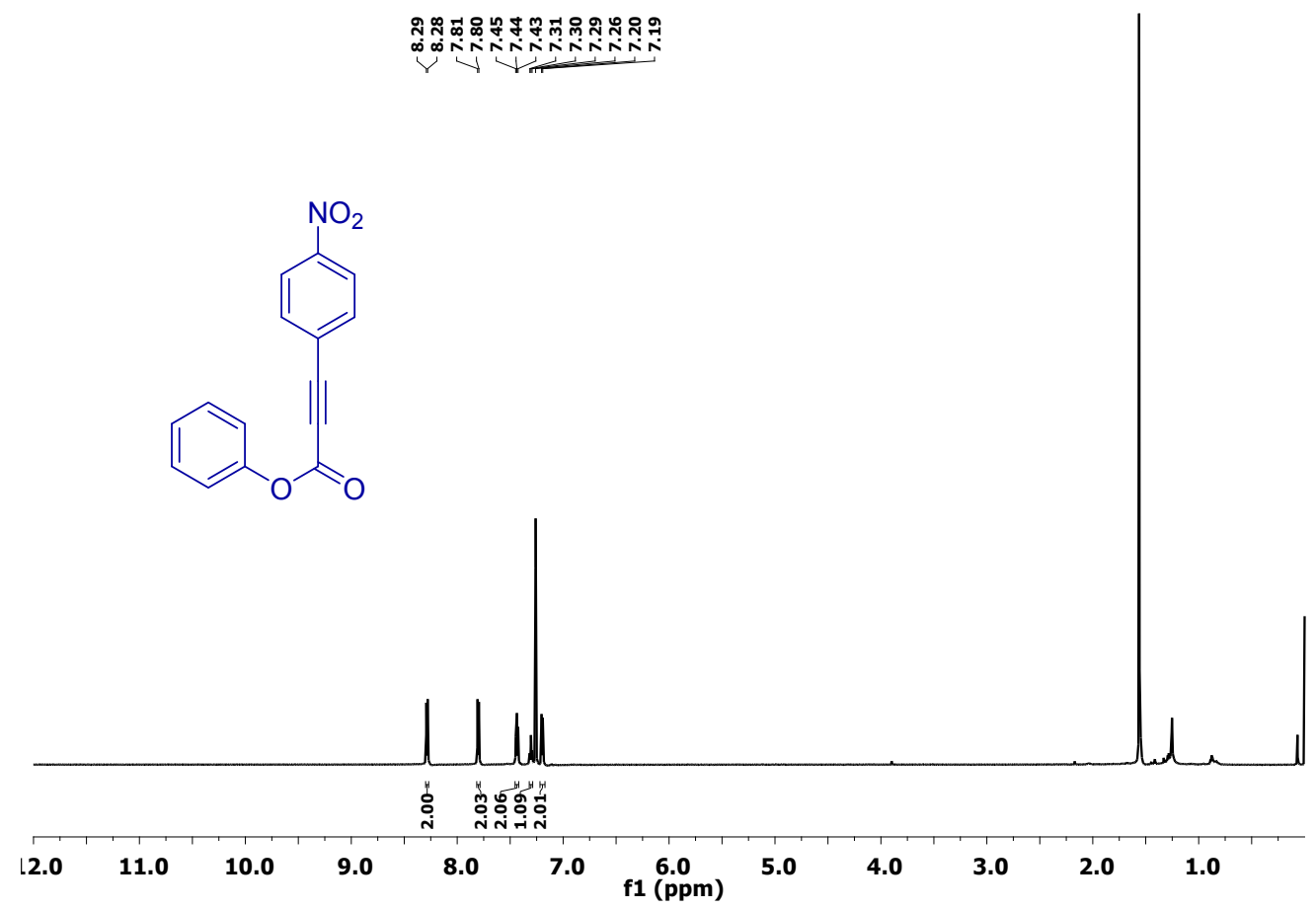

Fig. S102. ${ }^{1} \mathrm{H} \mathrm{NMR}\left(700 \mathrm{MHz}, \mathrm{CDCl}_{3}\right)$ spectrum of phenyl 3-(4-nitrophenyl)propiolate (3g). 


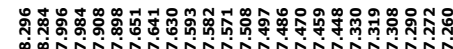
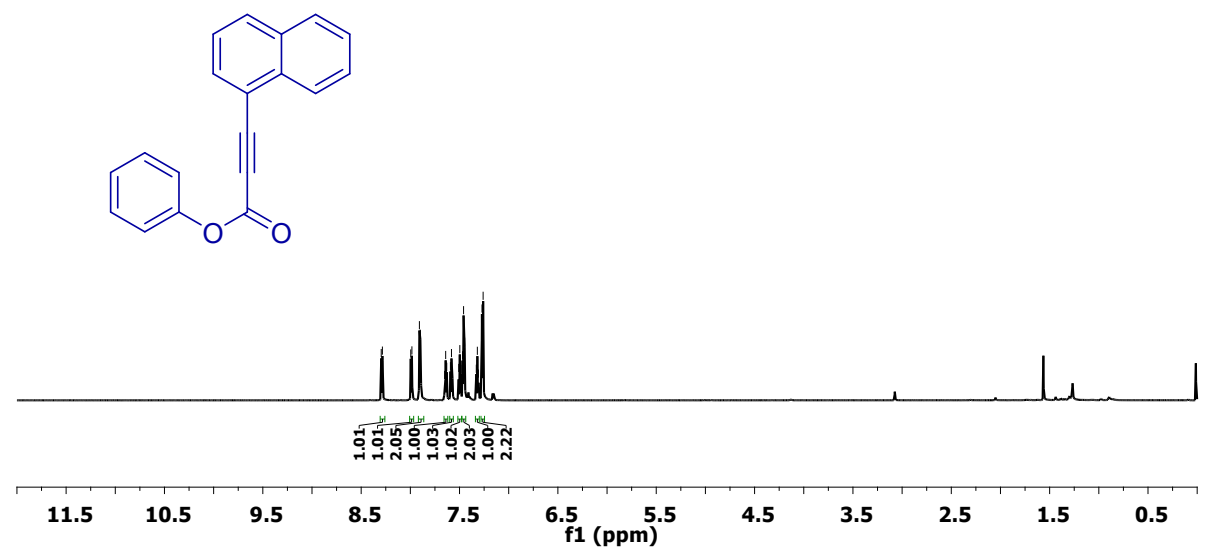

Fig. S103. ${ }^{1} \mathrm{H}$ NMR (700 MHz, $\mathrm{CDCl}_{3}$ ) spectrum of phenyl 3-(naphthalen-1-yl)propiolate (3h).
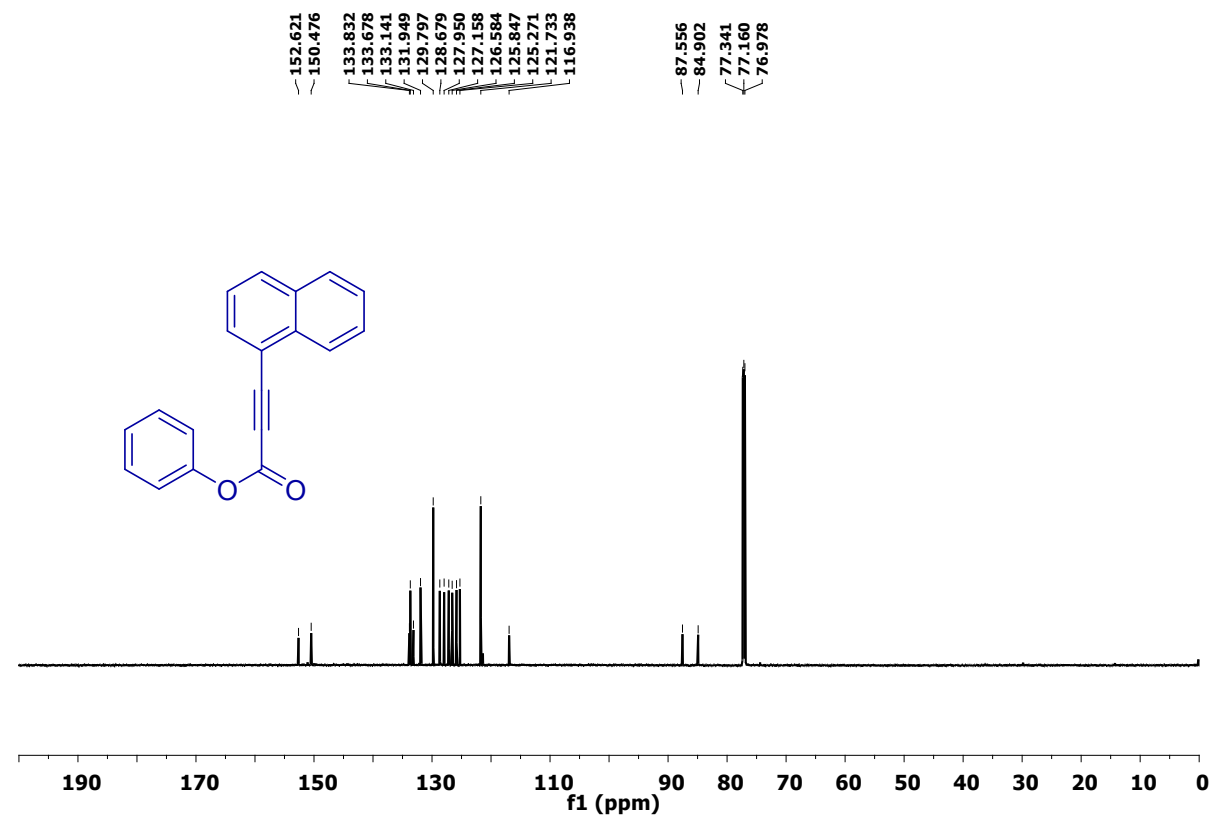

Fig. S104. ${ }^{13} \mathrm{C}$ NMR (175 MHz, $\left.\mathrm{CDCl}_{3}\right)$ spectrum of phenyl 3-(naphthalen-1-yl)propiolate (3h). 


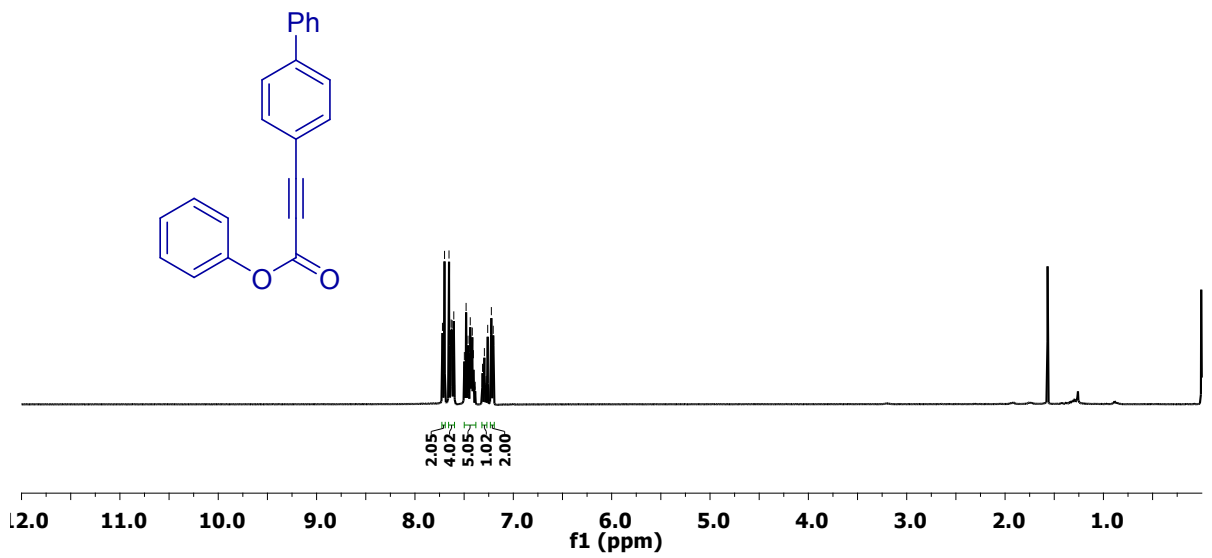

Fig. S105. ${ }^{1} \mathrm{H}$ NMR (400 MHz, $\left.\mathrm{CDCl}_{3}\right)$ spectrum of phenyl 3-([1,1'-biphenyl]-4-yl)propiolate (3i).

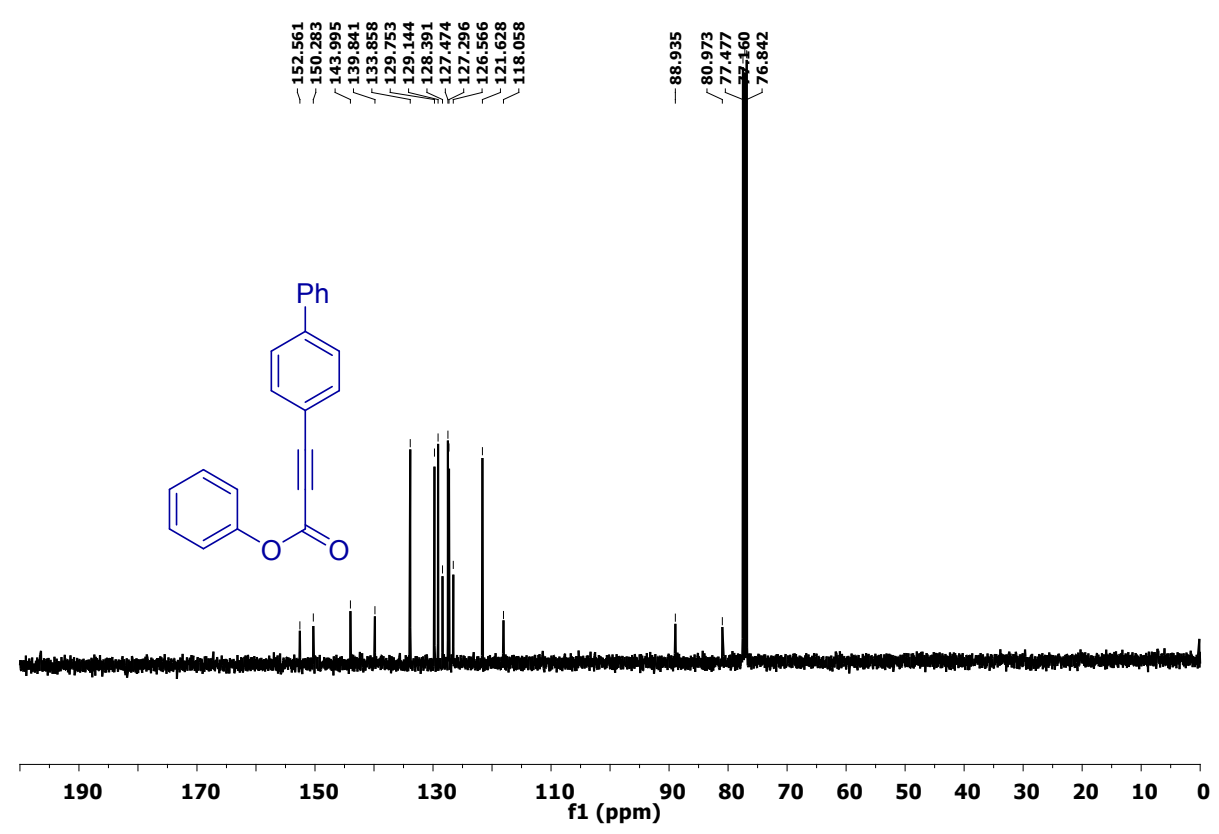

Fig. S106. ${ }^{13} \mathrm{C}$ NMR (100 MHz, $\left.\mathrm{CDCl}_{3}\right)$ spectrum of phenyl 3-([1,1'-biphenyl]-4-yl)propiolate (3i). 


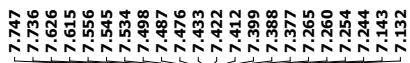

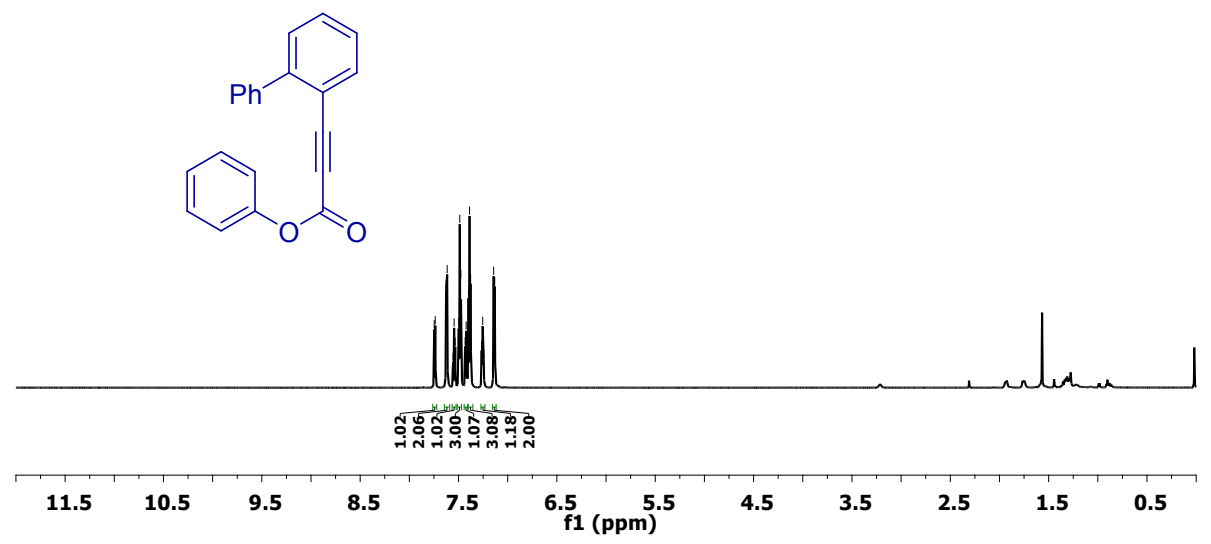

Fig. S107. ${ }^{1} \mathrm{H}$ NMR (700 MHz, $\left.\mathrm{CDCl}_{3}\right)$ spectrum of phenyl 3-([1,1'-biphenyl]-2-yl)propiolate $(3 \mathbf{j})$.
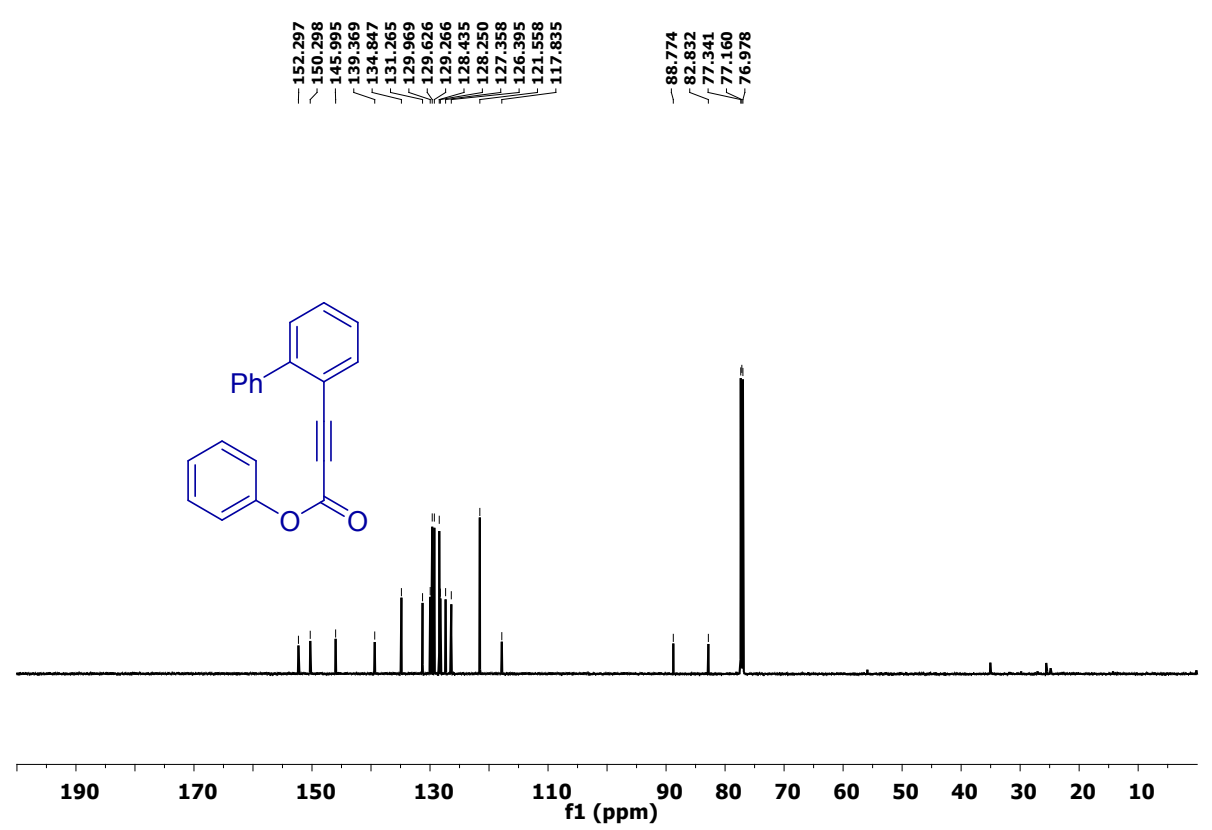

Fig. S108. ${ }^{13} \mathrm{C} \mathrm{NMR}\left(175 \mathrm{MHz}, \mathrm{CDCl}_{3}\right)$ spectrum of phenyl 3-([1,1'-biphenyl]-2-yl)propiolate (3j). 


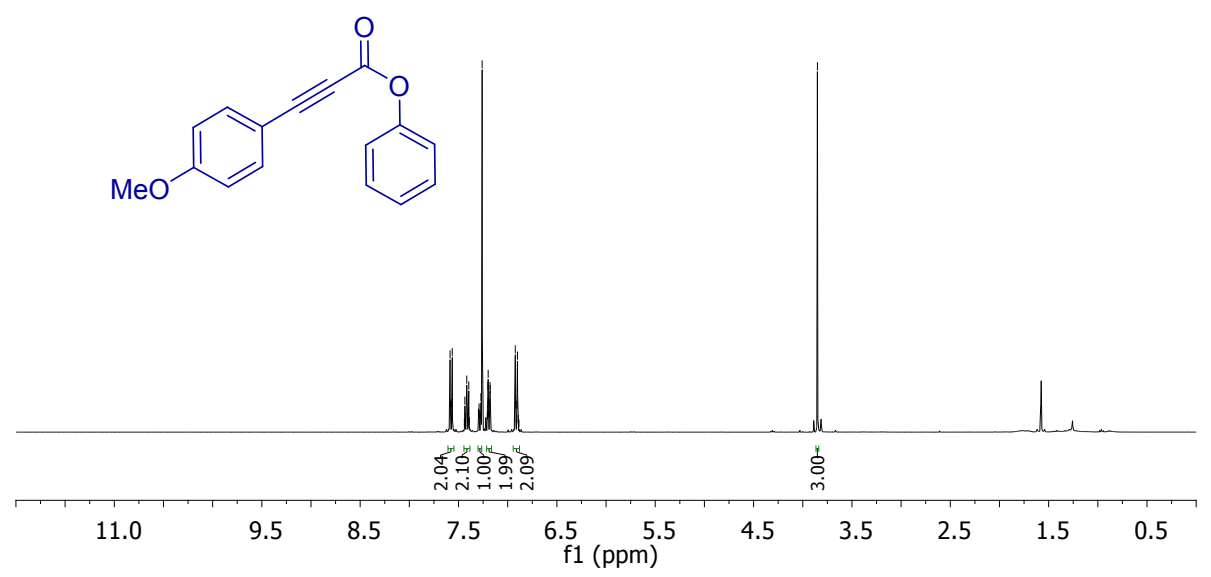

Fig. S109. ${ }^{1} \mathrm{H}$ NMR (700 MHz, $\mathrm{CDCl}_{3}$ ) spectrum of Phenyl 3-(4-methoxyphenyl)propiolate (3k).
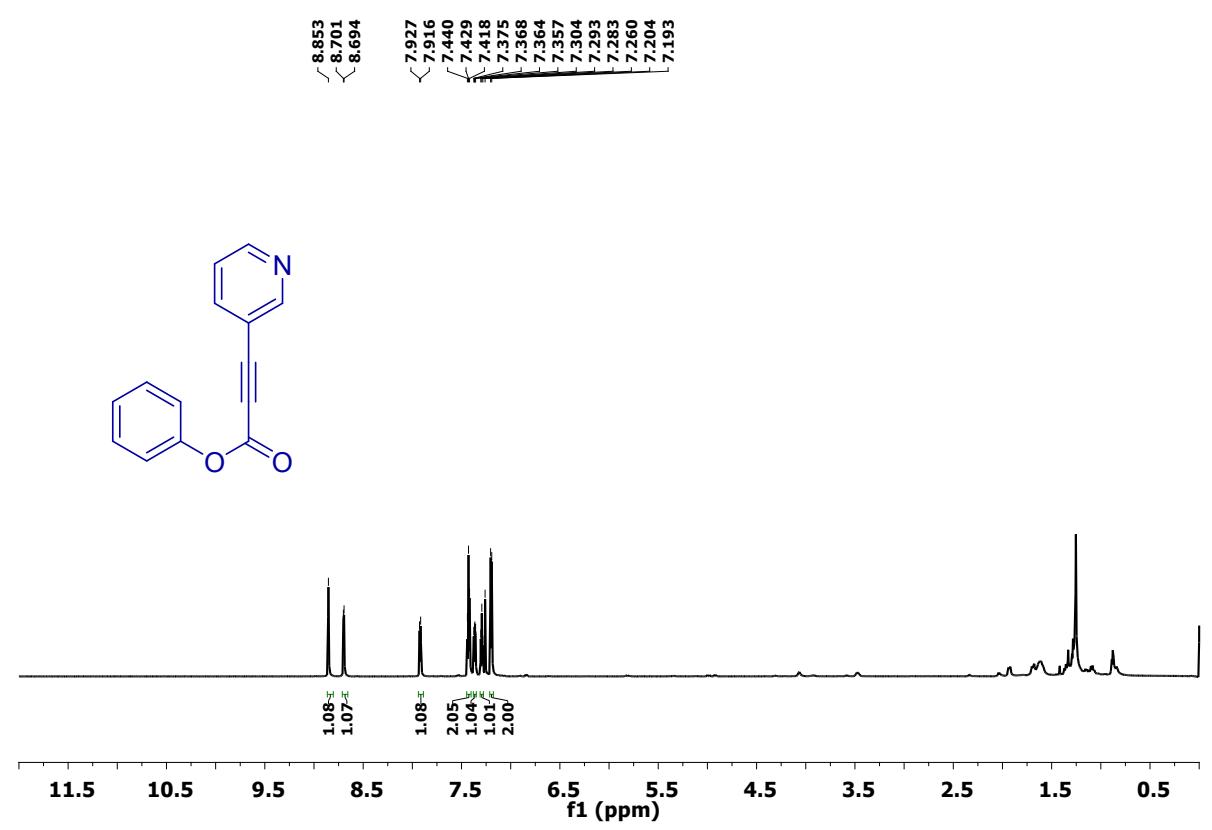

Fig. S110. ${ }^{1} \mathrm{H}$ NMR (700 MHz, $\mathrm{CDCl}_{3}$ ) spectrum of phenyl 3-(pyridin-3-yl)propiolate (3I). 


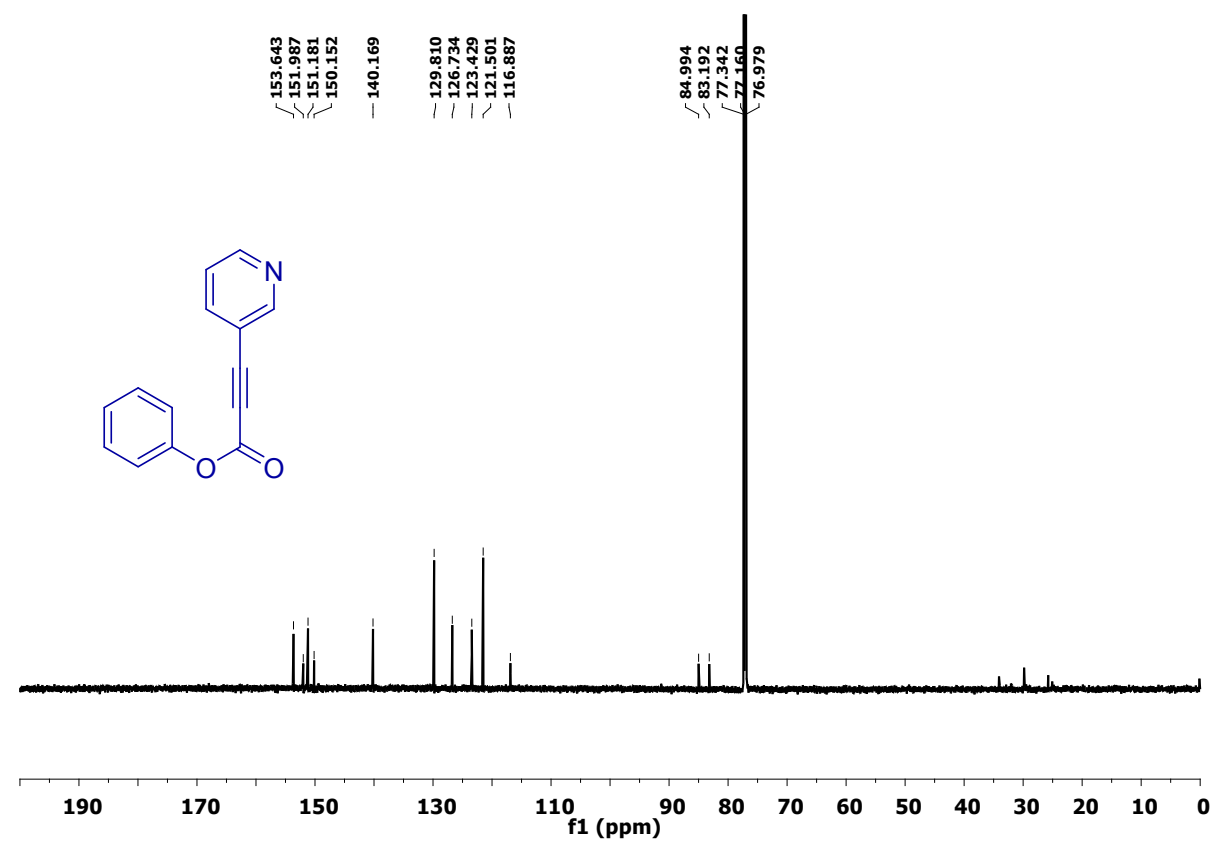

Fig. S111. ${ }^{13} \mathrm{C}$ NMR (175 MHz, $\mathrm{CDCl}_{3}$ ) spectrum of phenyl 3-(pyridin-3-yl)propiolate (3I).

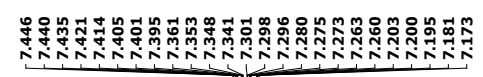
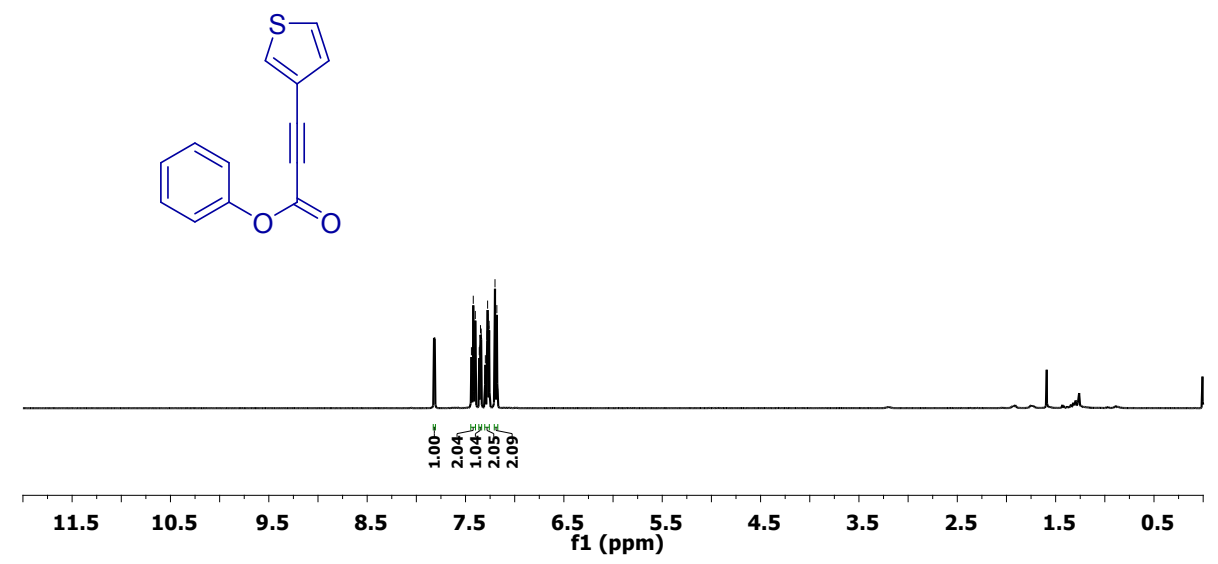

Fig. S112. ${ }^{1} \mathrm{H}$ NMR (400 MHz, $\left.\mathrm{CDCl}_{3}\right)$ spectrum of phenyl 3-(thiophen-3-yl)propiolate (3m). 

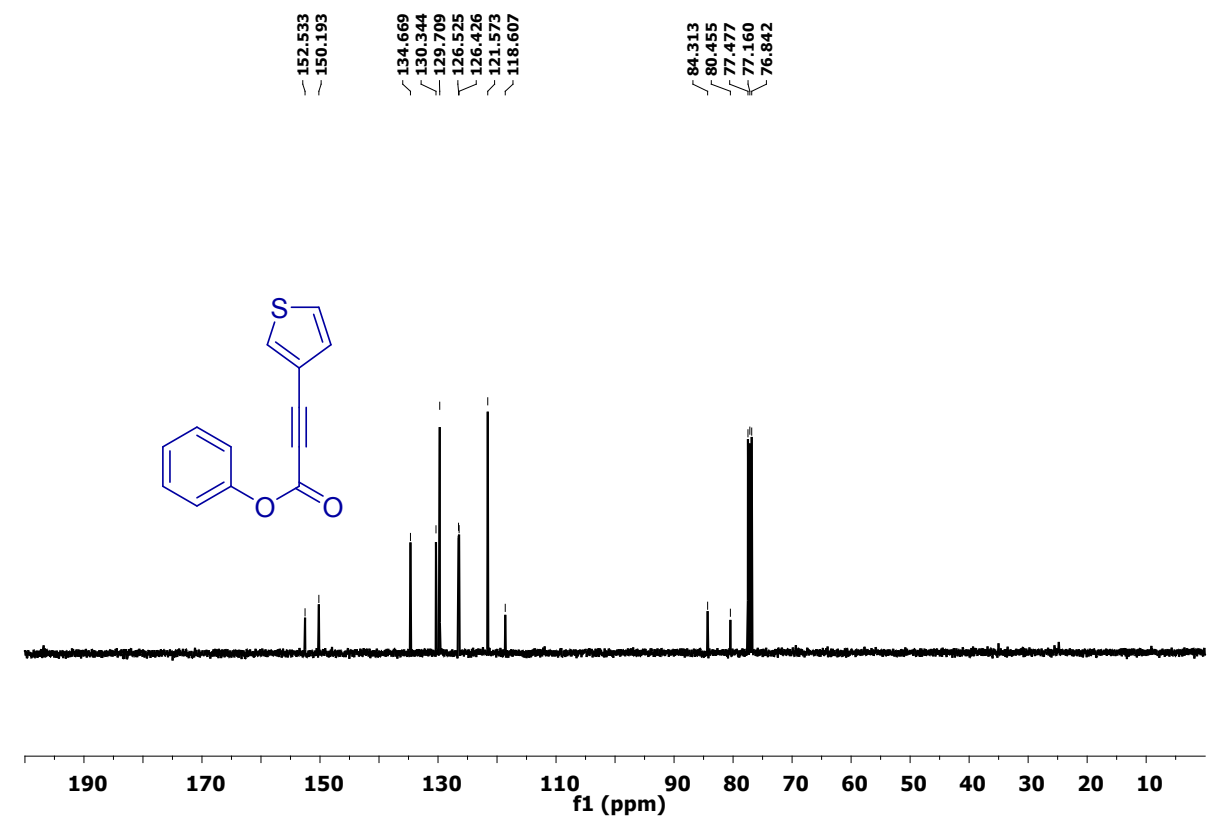

Fig. S113. ${ }^{13} \mathrm{C}$ NMR (100 MHz, $\mathrm{CDCl}_{3}$ ) spectrum of phenyl 3-(thiophen-3-yl)propiolate (3m).

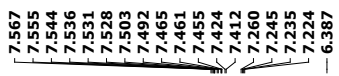

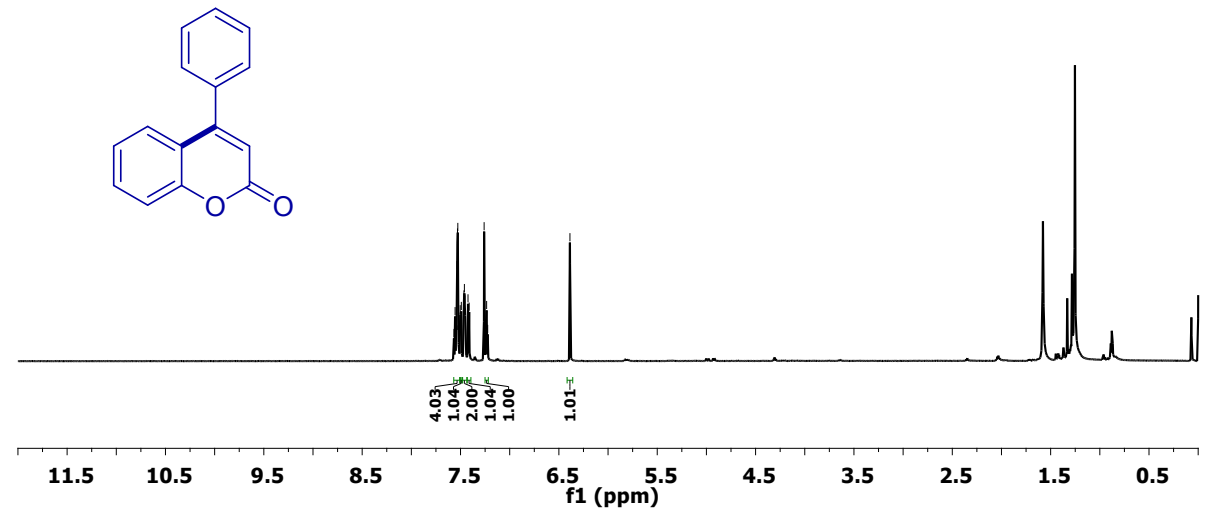

Fig. S114. ${ }^{1} \mathrm{H} \mathrm{NMR}\left(700 \mathrm{MHz}, \mathrm{CDCl}_{3}\right)$ spectrum of 4-phenyl-2H-chromen-2-one (5). 


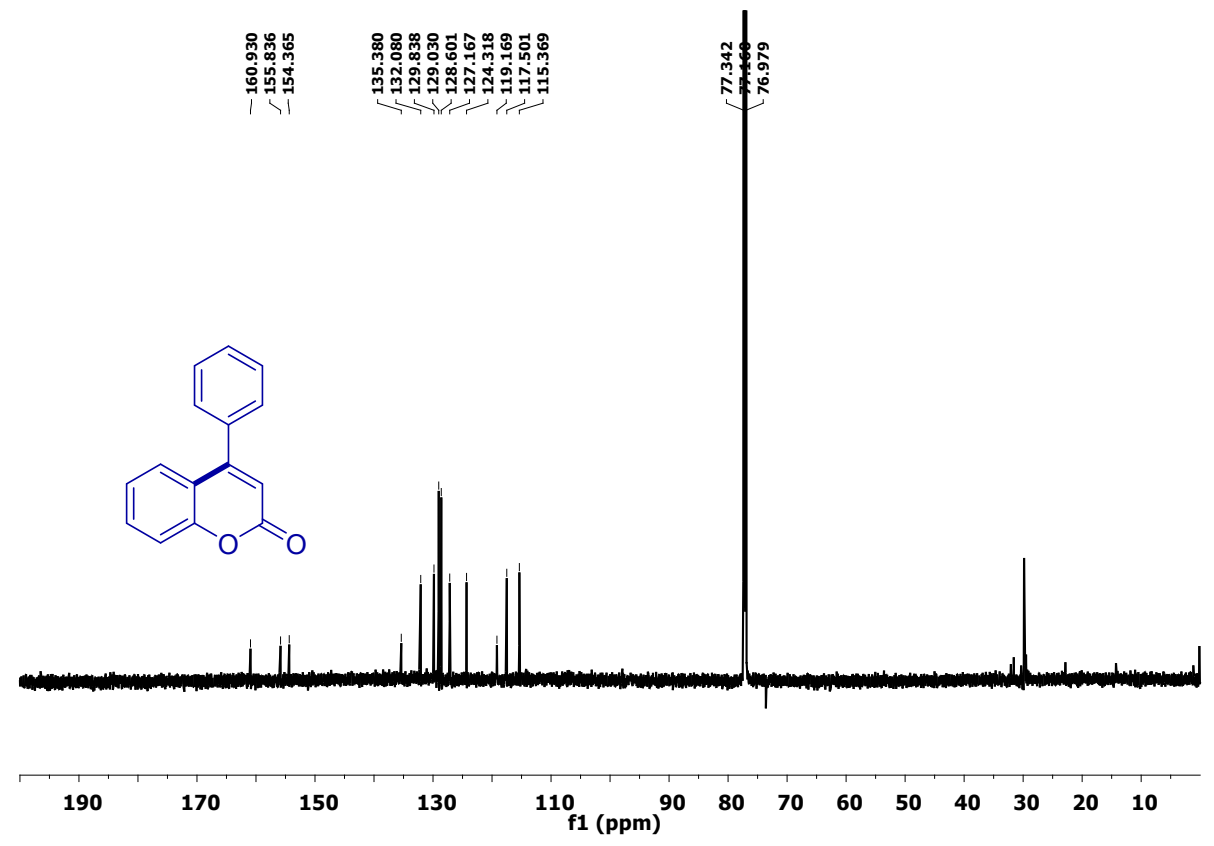

Fig. S115. ${ }^{13} \mathrm{C}$ NMR (175 MHz, $\mathrm{CDCl}_{3}$ ) spectrum of 4-phenyl-2H-chromen-2-one (5).
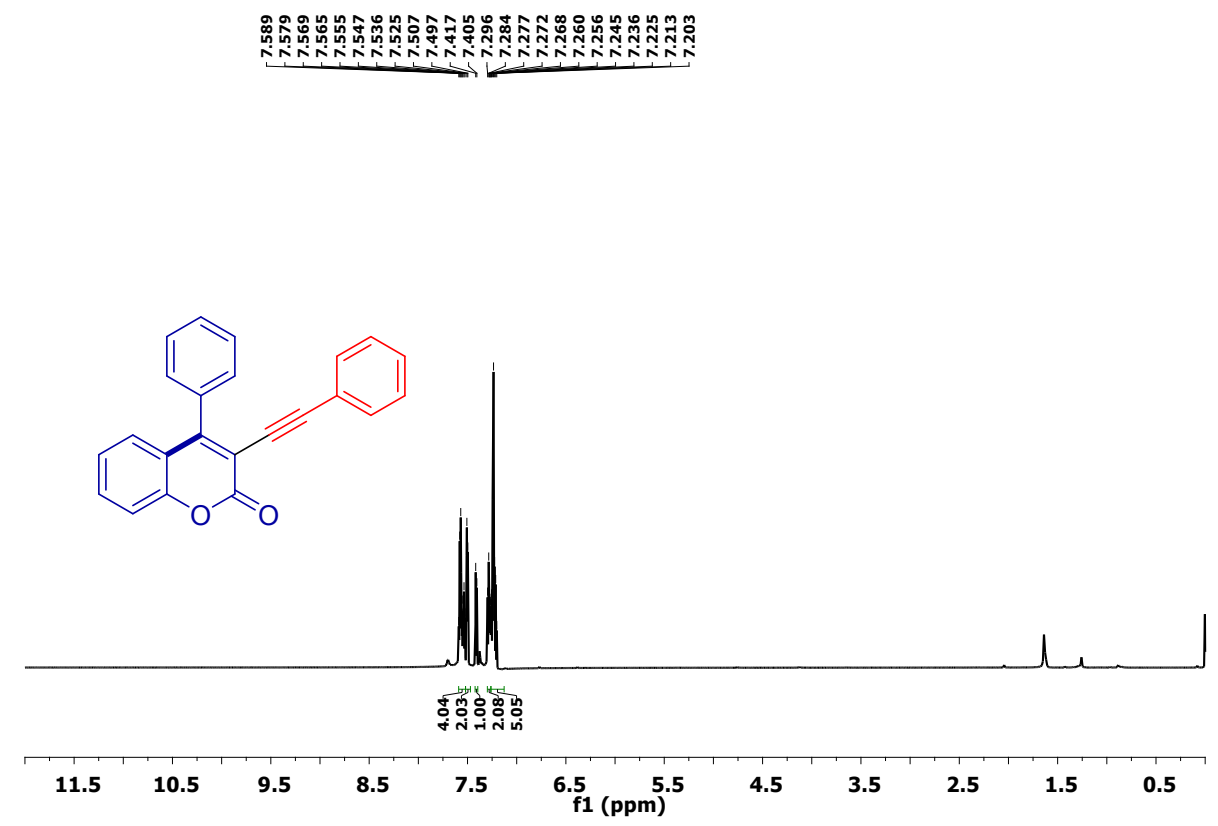

Fig. S116. ${ }^{1} \mathrm{H}$ NMR (700 MHz, $\mathrm{CDCl}_{3}$ ) spectrum of 4-phenyl-3-(phenylethynyl)-2H-chromen-2one (6). 


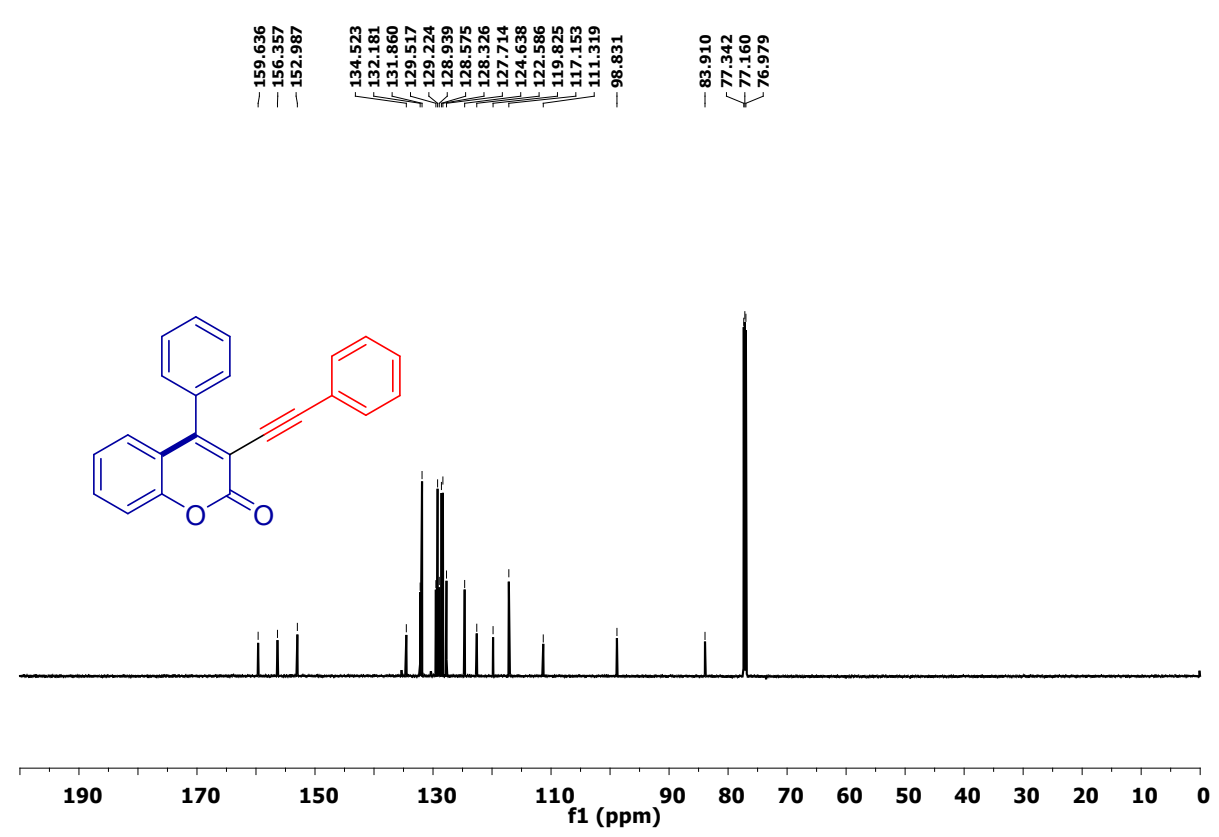

Fig. S117. ${ }^{13} \mathrm{C}$ NMR (175 MHz, $\mathrm{CDCl}_{3}$ ) spectrum of 4-phenyl-3-(phenylethynyl)-2H-chromen2-one (6).

\section{Unsuccessful Substrates.}
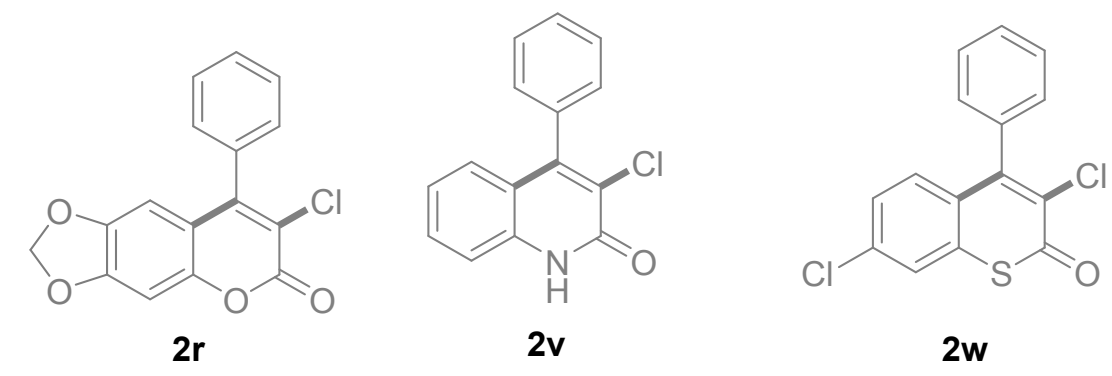

Chart 1. Reaction was unsuccessful for the above substrates. 


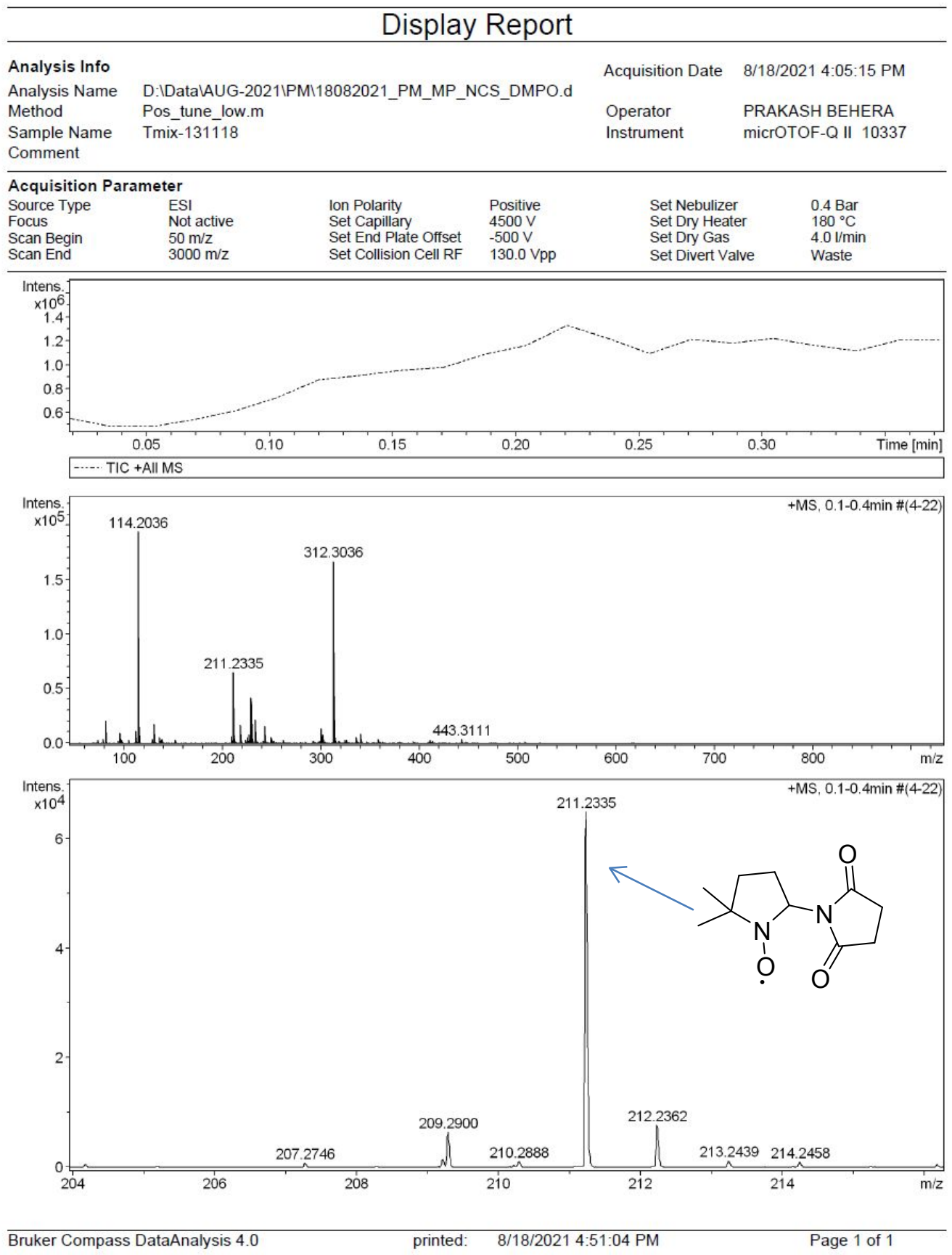

Fig. S118. ESI-MS data of the experiment using DMPO under standard condition for EPR study. 
Description of Light Source. Blue LEDs were purchased from market available commercial source (CLAIR AREVIK BRIGHT PLUS Pvt. Ltd. INDIA). The wave length was 450-455 nm (measured by BWTEK spectrometer). Quartz glass (brand name: Luzchem) was used as reaction vessel. Distance between light source and quartz tube was approximately $4 \mathrm{~cm}$ and no filter was used for the reaction. High speed electric fans were used to ventilate the area by maintaining the room temperature $\leq 30^{\circ} \mathrm{C}$.

\section{Photochemical reaction set up.}

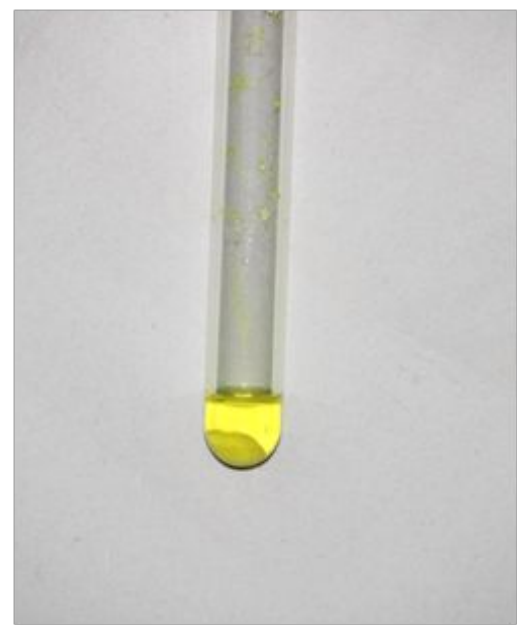

before reaction

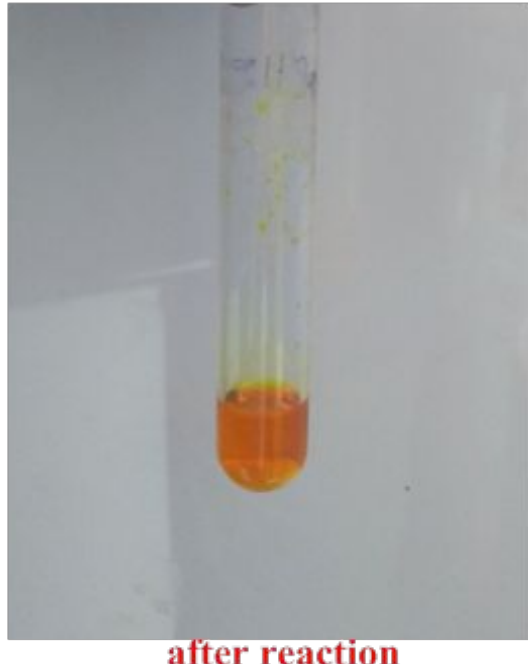

after reaction

Fig. S119. Color before and after of the reaction. 


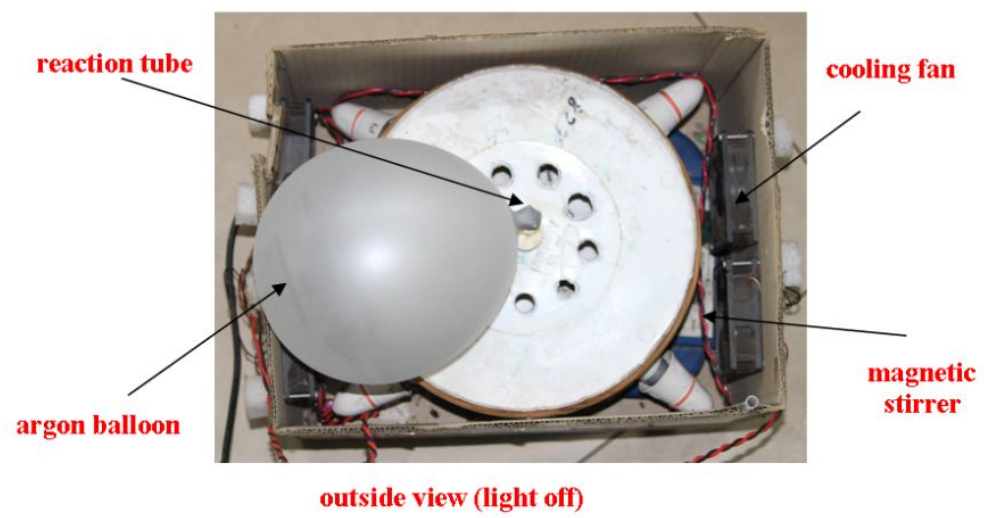

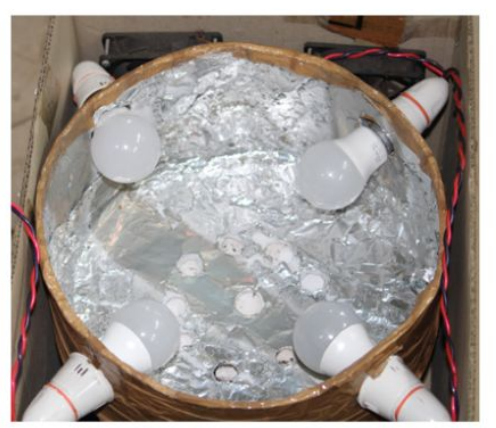

inside view

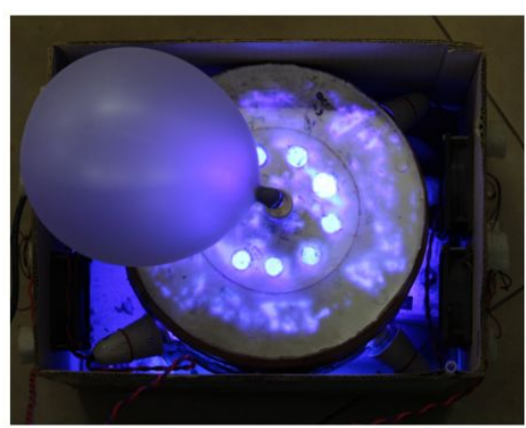

outside view (light on)

Fig. S120. The photo-reactor. 